\title{
A COMPUTATIONAL STUDY OF PHASE SEPARATION IN POLYMER SOLUTIONS UNDER A DOUBLE QUENCH
}

by

\author{
Ehsan Hosseini \\ Bachelor of Chemical Engineering \\ Azad University, Iran, 2001
}

\author{
A thesis \\ presented to Ryerson University \\ in partial fulfillment of the \\ requirements for the degree of \\ Master in Applied Science \\ in the program of \\ Chemical Engineering
}

Toronto, Ontario, Canada, 2009

(C)Ehsan Hosseini, 2009 


\section{Acknowledgements}

I would like to express my deepest gratitude towards my supervisor, Dr. Philip K. Chan, for giving me the opportunity to work on this research project, for his valuable guidance and encouragement throughout the course of this thesis.

In addition, my sincere gratitude goes out to Dr. Philip K. Chan for providing me with a stipend during the extended period of my studies and also for providing me the excellent research environment, furthermore for his patience and understanding during my difficult times.

Thanks to Ryerson University School of Graduate Studies for providing me with scholarship during my studies.

Special thanks to my friends M. Tabatabaei and M. Farrokhi for their advice and assistance.

I would also like to express my deepest appreciation to my parents for their encouragement and to my wife Negar, for her patience and support. 


\section{Abstract}

\section{A COMPUTATIONAL STUDY OF PHASE SEPARATION IN POLYMER SOLUTIONS UNDER A DOUBLE QUENCH}

Ehsan Hosseini, MASc, 2009.

Department of Chemical Engineering, Ryerson University

350 Victoria Street, Toronto, Ontario M5B 2K3 Canada.

Polymer-dispersed liquid crystals (PDLCs) are a relatively new class of materials used for many applications ranging from switchable windows to projection displays. PDLCs are formed by spinodal decomposition induced by thermal quenching or polymerization. The objective of the present study is to introduce a new mechanism of phase separation in a binary polymer solution and develop a mathematical model and computer simulation to describe the phase separation during the early and intermediate stages of nucleation and growth and spinodal decomposition induced by thermal double quenching. The equilibrium limits of phase separation as well as phase transition are calculated by taking into consideration the Flory-Huggins theory for the free energy of mixing. A two step quench is modeled using Cahn-Hilliard theory for asymmetric binary polymer solution which is quenched from a stable state in the one-phase region to a metastable region where nucleation and growth occurs. The solution is allowed to coarsen for different time periods before a second quench was applied to a point further inside the phase diagram. The numerical results in two dimensions replicate the experimental and numerical work that has been recently done and published. 


\section{Table of Contents}

Author's Declaration ....................................................................................................................... ii

Acknowledgements ......................................................................................................................... iii

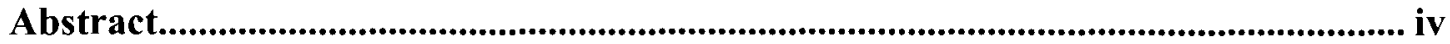

Table of Contents ...................................................................................................................... v

List of Figures............................................................................................................................. viii

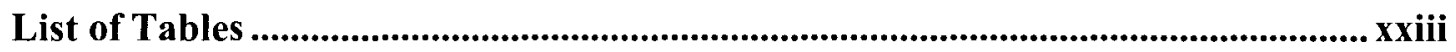

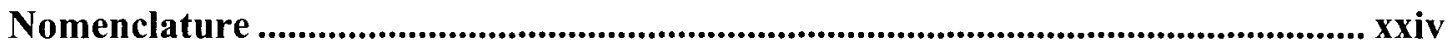

\section{Chapter 1}

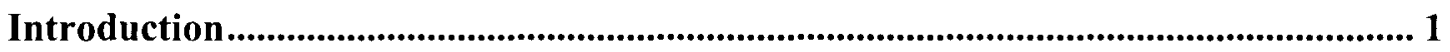

1.1 Phase Separation in Polymer Solutions ……............................................ 1

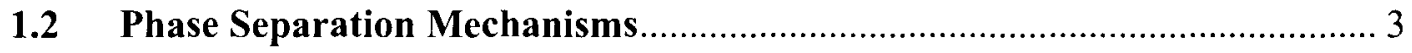

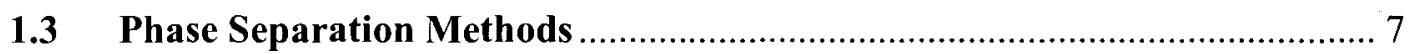

1.3.1 Thermally-Induced Phase Separation Method................................................ 10

1.3.2 Polymerization-Induced Phase Separation Method ....................................... 12

1.3.3 Solvent-Induced Phase Separation Method ................................................... 15

1.3.4 Reaction-Induced Phase Separation Method ................................................. 15

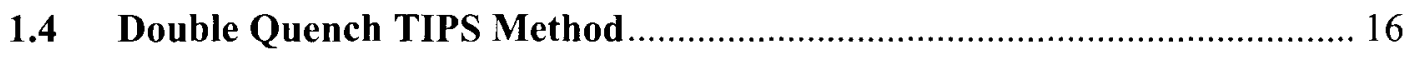

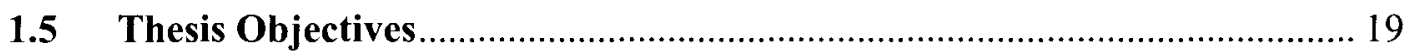

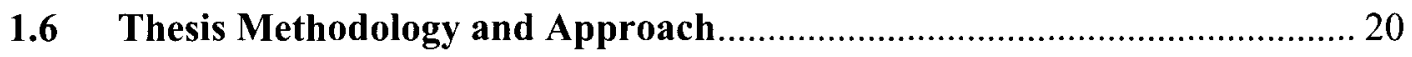

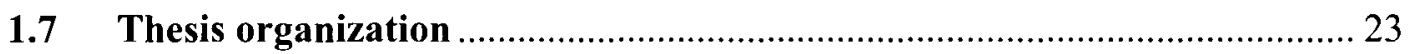




\section{Chapter 2}

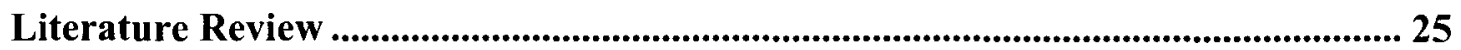

2.1 Polymer Dispersed Liquid Crystal (PDLC) ....................................... 25

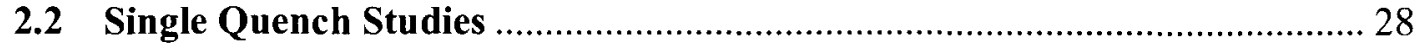

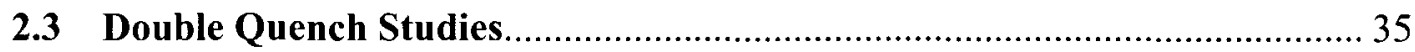

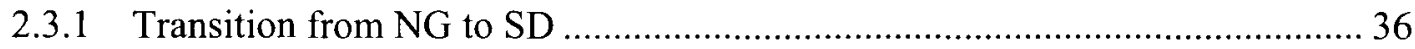

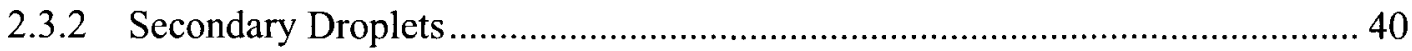

2.3.2.1 Critical Double Quench Mechanism....................................................... 56

2.3.2.2 Off-Critical Double Quench within SD Mechanism ............................... 59

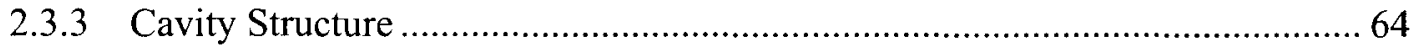

\section{Chapter 3}

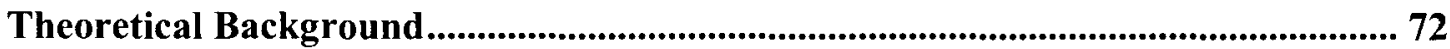

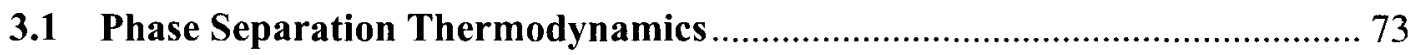

3.1.1 Flory-Huggins Theory ............................................................................. 77

3.1.1.1 Entropy of Mixing of Polymer Solutions................................................ 78

3.1.1.2 Enthalpy of Mixing of Polymer Solutions ................................................. 79

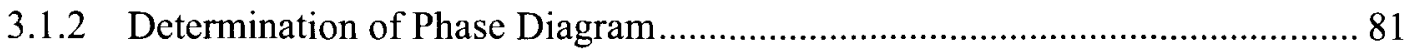

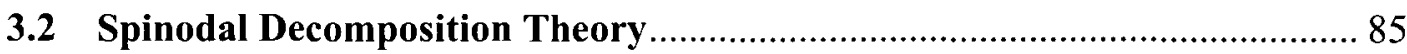

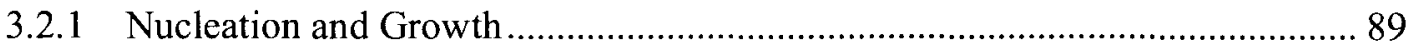

3.2.2 Spinodal Decomposition vs. Nucleation and Growth..................................... 92

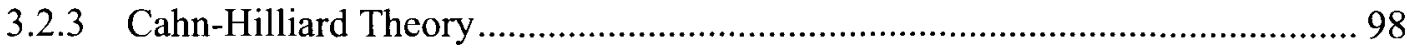


3.2.3.1 Noise Term

3.2.3.2 Concentration Dependent Mobility and Mutual Diffusion 110

\section{Chapter 4}

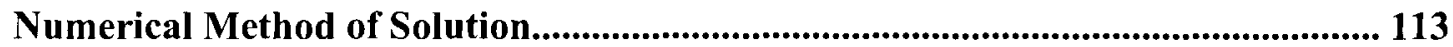

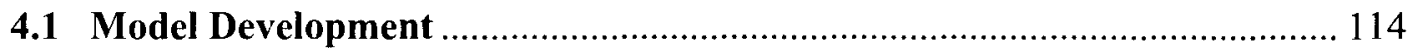

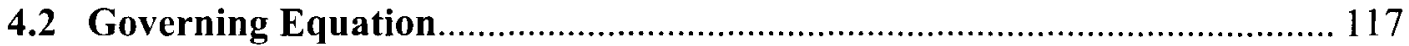

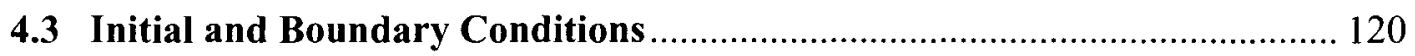

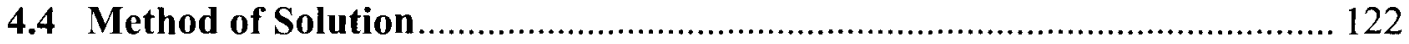

\section{Chapter 5}

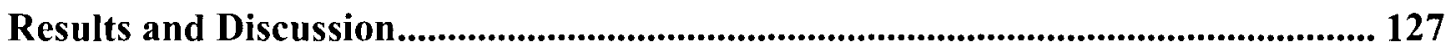

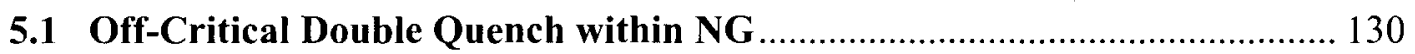

5.2 Structure Factor Evolution in Shallow Double Quench.............................. 147

5.3 Structure Factor Evolution in Deep Double Quench ................................. 152

5.4 Structure Factor Growth from NG to SD ................................................. 155

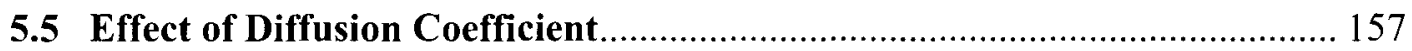

\section{Chapter 6}

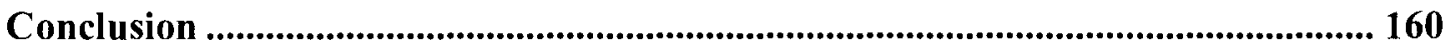

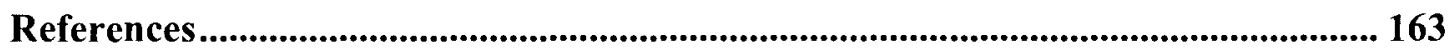

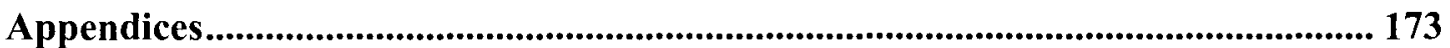




\section{List of Figures}

Figure 1.1: Schematic phase diagram of a binary asymmetric polymer solution .2

Figure 1.2: Schematic illustration of phase separation by spinodal decomposition mechanism (a) one-dimensional evolution of concentration profiles; (b) two-dimensional picture of the resultant structure. $c_{\alpha}$ and $c_{\beta}$ are the high and low equilibrium solvent concentration on the binodal curve respectively 4

Figure 1.3: Schematic illustration of phase separation by spinodal decomposition mechanism (D to $\mathrm{E}$ or $\mathrm{A}$ to $\mathrm{C}$ ) and nucleation and growth (A to $\mathrm{B})$ in thermally-induced phase separation process, showing metastable and unstable region with binodal

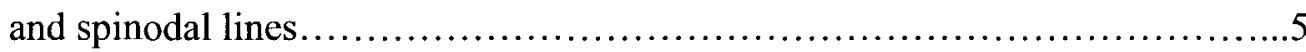

Figure 1.4: Schematic illustration of phase separation by nucleation and growth mechanism: (a) one-dimensional evolution of concentration profiles; (b) two-dimensional picture of the resultant structure. $c_{\alpha}$ and $c_{\beta}$ are the high and low equilibrium solvent concentration on the binodal curve respectively 8

Figure 1.5: TIPS process taken at three different times for PDLC. The droplets grow in size as time passes .10 
Figure 1.6: A temperature vs. composition phase diagram of TIPS process showing the various types of quenching into spinodal region, off-critical (B and D) and critical (C). Route $\mathrm{A}$ is a quench into metastable region. .12

Figure 1.7: Schematic phase diagram of PIPS process. The solid (dashed) curves represent the binodal (spinodal) lines. $c_{0}$ and $T_{i}$ are average concentration and initial temperature respectively. $N_{2}$ is the polymer degree of polymerization which increases with polymerization. Note that the phase diagram becomes asymmetric and the system point is thrust into the unstable region where phase separation occurs by spinodal decomposition 14

Figure 1.8: Schematic phase diagram of TIPS process. The solid (dashed) curves represent the binodal (spinodal) lines. $T_{c}$ and $c_{c}$ are critical temperature and concentration respectively. In case (a) the solution is critically quenched into A first and then to $\mathrm{B}$ or $\mathrm{C}$ featuring shallow and deep quenches while in case (b); the solution is quenched off-critically into D first and then to $\mathrm{E}$ and $\mathrm{F}$ for shallow and deep quenches 17

Figure 1.9: The theory, computer simulation and experiment [77]. Copyright Springer Kluwer, Academic Publishers, 1998 .21

Figure 1.10: Computational modeling flowchart of the thesis objectives. .22 
Figure 2.1: Schematic illustration of a PDLC cell showing the scattered (transmitted) light in off-state (on-state) .27

Figure 2.2: Temporal change in the patterns observed with optical microscopy during the SD-type off-critical quench in phase separation process [7] 30

Figure 2.3: Dimensionless concentration spatial patterns formed during the SD-type phase separation phenomena for the simulation performed by Chan [12] corresponding to (a) off-critical and (b) critical quench. The black regions (white regions) in the patterns represent solvent-rich (solute-rich) regions. For an off-critical (critical) quench simulation, a droplet (interconnected) morphology structure forms $[12]$ 31

Figure 2.4: Interconnected pattern observed with optical microscopy during the SD-type critical quench in phase separation process [24]. 33

Figure 2.5: Schematic phase diagram of a binary mixture (temperature vs. concentration). The solid curve is the binodal line (the coexistence curve) and the dashed curve the classical spinodal line. The shaded area is a transitional region from metastability to instability. The broadness of the transition depends on the interaction range. The arrow indicates an off-critical quench condition. $\phi_{c o e x}^{(1)}$ and $\phi_{c o e x}^{(2)}$ are the two branches of the coexistence curve [40] .38 
Figure 2.6: UCST-type phase diagram and two-step temperature jump: the first jump from A to B and the second from B to C (shallow) or B to D (deep): (-) binodal and (---) spinodal curves [52]

Figure 2.7: Backscattered electron image of the phase-separation structure formed in the two-step temperature jump of blend $\left(1.35 \times 10^{4} \mathrm{~s}\right.$ after the second temperature jump). White regions correspond to the poly(2-chlorostyrene)-rich phase [59].

Figure 2.8: Pattern evolution caused by a double quench: $\left(6.8^{\circ} \mathrm{C}, 600 \mathrm{~s}, 21.4^{\circ} \mathrm{C}\right)\left(163.0^{\circ} \mathrm{C}\right.$, $168.0^{\circ} \mathrm{C}$ ) in a PS-PVME (50-50) mixture; (a) $60 \mathrm{~s}$, (b) $120 \mathrm{~s}$, (c) $240 \mathrm{~s}$, (d) $480 \mathrm{~s}$, (e) $720 \mathrm{~s}$, and (t) $960 \mathrm{~s}$ after the second quench [86]. 44

Figure 2.9: Various kinds of double-quench sequences consisting of a first deeper quench and a subsequent deeper quench. In case $a$, both phases become metastable. In case $b$, one phase becomes metastable, while the other unstable. In case $c$, both phases become unstable. In case $d$, phase inversion is induced by the second quench [86] .45

Figure 2.10: Two dimensional pattern evolution during bi-continuous spinodal decomposition for a symmetric composition under a high-fluidity condition at different time steps [87]. 46 
Figure 2.11: Phase diagram of a binary mixture of $\mathrm{dPB}-\mathrm{hPI}$. The solid and dotted curves correspond, respectively, to the binodal and spinodal lines. The two-step temperature jump was performed according to the scheme indicated by arrows. (b) Thermal history applied to the two-step phase separation experiments. The first-step SD process at $T_{1}\left(542{ }^{\circ} \mathrm{C}\right)$ lasted for $t_{0}$ minutes and subsequently the second-step $T$-jump from $T_{1}$ to $T_{2}\left(570{ }^{\circ} \mathrm{C}\right)$ was performed. The two phases developed after the first-step SD was brought again in spinodal region for the second-step phase separation process [90] .47

Figure 2.12: Pattern evolution of the OCL/OS mixture. Secondary droplets are more clearly observed for a deeper quench of nearly symmetric mixtures and never observed for off-symmetric mixtures having droplet morphology under any quench conditions. This can be easily confirmed by comparing Figs. 2(al)-2(a3) with Figs. 2(cl)-2(c3) [88] .48

Figure 2.13: Phase diagram illustrating the two-step quench phase separation process in a symmetric binary polymer blend $[50]$. .50

Figure 2.14: Development of the polymer morphology following the second deep quench into the two-phase region [50] .51 
Figure 2.15: The phase diagram of the OS/OCL mixture. The solid and dashed curves are the binodal and spinodal lines, respectively. The dashed line is the composition

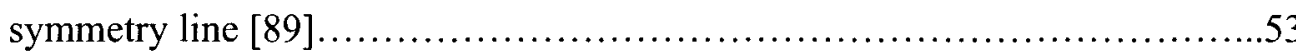

Figure 2.16: Temporal change of the pattern inside a large OCL-rich droplet after the second quench. The size of an image corresponds to $25 \times 25 \mu \mathrm{m}$ [89] .54

Figure 2.17: Schematic representation of TIPS in a two-step critical quench in a symmetric polymer solution. Both polymer and phase separated liquid crystals obtained from the first quench undergo another phase separation independently. 58

Figure 2.18: Schematic representation of TIPS in a two-step off-critical quench in a symmetric polymer solution. Both polymer and phase separated liquid crystals obtained from the first quench undergo another phase separation independently

Figure 2.19: Temporal change in the structure factor of the second order structure alone 62

Figure 2.20: Schematic figure describing the pattern evolution after a double quench. The thick black arrow indicates the diffusion flux induced by the concentration gradient [89] 
Figure 2.21: SEM micrographs of PS/PEG200 (70/30) (w/w) cast from $90 \mathrm{wt} \%$ toluene solution surface micrograph (notice the cavity structure) [63] .64

Figure 2.22: Pictures taken with a microscope in the visual observation mode to explain the formation of the core-shell structure [63] .65

Figure 2.23: Development of the polymer morphology following a second shallow (a) and deep quench (b) (notice the cavities inside the droplets for deep quench at $\left.\tau_{2}=10\right)[78]$

Figure 2.24: Development of the morphology following the quench into the spinodal region. To highlight the existence of cores rich in component A, only a selected $128 \times$ 128 area of the simulation, corresponding to the boxed region in (a) is shown in (b) to (d). The grey scale range, which is the same for all snapshots, is such that white represents regions of pure $\mathrm{A}$, and black represents regions of pure $\mathrm{B}$ [49] .68

Figure 2.25: TEM (transmission electron microscopy) micrograph of cavity microsphere [136]. The cavities inside the droplets are clearly evident. 69

Figure 2.26: Morphology of polymerized PMMA/epoxy 50/50 blends, start temperature of polymerization profile: $60^{\circ} \mathrm{C}[138]$ .70 
Figure 3.1: Schematic diagram of Gibbs free energy of mixing as a function of polymer concentration [64]..... .74

Figure 3.2: Schematic of phase behavior in polymer solutions. Shaded areas represent the two-phase regions and the un-shaded areas represent one-phase regions [105] .77

Figure 3.3: Experimentally determined phase diagram of the PCL/PS blend by Tanaka and Nishi [53]. The black circles are the experimental data for the melting temperature and the white circles are the phase separation temperatures obtained from cloud point measurements. Solid and dashed curves are computed using the Flory-Huggins mixing theory [105] .82

Figure 3.4: Schematic phase diagram with corresponding the free energy of isotropic mixing and its derivative at a certain temperature [107] 84

Figure 3.5: Typical phase diagram of a symmetric polymer mixture for the TIPS process. The arrow (a) indicates a temperature change from the homogeneous region into the metastable region (NG) and arrows (b) and (c) represent temperature jumps into the unstable region (SD) off-critically and critically respectively. The solid (dotted) curve represents binodal (spinodal) line 86 
Figure 3.6: SD-type phase separation droplet (interconnected) morphology resulting from off-critical (critical) temperature quench. .87

Figure 3.7: The three stages of SD plotted as concentration vs. distance, (a) early stage, (b) intermediate stage and (c) late stage [32] 88

Figure 3.8: Illustration of the nucleation and growth phase separation mechanism at (a) early, (b) intermediate and (c) final stages. At stage (b) the nuclei form and grow with time leading to the final structure in (c) where many length scales are obtained .90

Figure 3.9: Temperature vs. volume fraction phase diagram illustrating the tie lines in TIPS method 91

Figure 3.10: Illustration of the spinodal decomposition phase separation mechanism at (a) early, (b) intermediate and (c) final stages.

Figure 3.11: Schematic representation of the time-evolution of the scattered light intensities with time and angle (or wave number $q$ ) during spinodal decomposition. The spinodal ring that forms becomes more intense and moves to smaller angles with time. The scattering maximum in the angular dependence corresponds to the location of the ring [93]. 95 
Figure 3.12: Schematic representation of the time-evolution of the scattered light intensities with time and angle (or wave number $q$ ) during nucleation and growth. In contrast to spinodal decomposition, ring formation is not observed and the scattered light intensities do not pass through a maximum [93].... .96

Figure 3.13: Growth rate $R(k)$ vs. the wavevector $k$. Following the linear theory, $R(k)$ is positive for $k<k_{c}$ and exhibits a maximum at $k=k_{m}[108]$ 108

Figure 3.14: A typical plot of the intensity $I(q, t)$ vs. scattering vector $q$, for studying phase separation by SD with time. 104

Figure 3.15: Schematic representation of the relationship for droplet diameter $d_{m}$ and wavelength $\lambda$ in two dimensions. $c$ is the average concentration. The circle represents a polymer droplet, since the droplet diameter equals half of the wavelength $d_{m} \propto \lambda / 2$ .105

Figure 3.16: Typical pattern for the noiseless case (a), noisy case (b) and strongly noisy (c) 107

Figure 3.17: Scaled scattering function $S(k, t)(\langle k\rangle(t))^{2}$ as a function of $k /\langle k\rangle(t)$ for the noiseless and noisy cases. The circles (tiny crosses) are data from the noiseless (noisy) case at different times. Though it is not evident on the scale of this 
figure, for the noiseless case, the tail of the curve drops off faster than $x^{-3}$

Figure 5.1: Schematic phase diagram of TIPS process. The solid (dashed) curves represent the binodal (spinodal) lines. In case (a); the solution undergoes a critical quench into $\mathrm{G}$ first and then to $\mathrm{H}$ or I for shallow or deep quench respectively, while in case (b); the solution undertakes a critical quench into $\mathrm{J}$ first and then to $\mathrm{K}$ and $\mathrm{L}$ for shallow and deep quenches respectively

Figure 5.2: The evolution of the dimensionless spatial concentration profile for the (a) shallow and (b) deep two-step phase separation. The initial average concentration is $c_{0}^{*}=0.5$, and the dimensionless diffusion coefficient is $D^{*}=2 \times 10^{5}[25]$

Figure 5.3: Schematic representation of a PDLC phase diagram curvature from symmetric state into asymmetric one [94]

Figure 5.4: Schematic representation of TIPS in a two-step off-critical quench in an asymmetric polymer solution. The solid (dashed) line represents the binodal (spinodal) curve 132

Figure 5.5: Dimensionless $2 \mathrm{D}$ contour spatial concentration profiles for $c^{*}\left(x^{*}, y^{*}\right)$ (left column) and 3D concentration fluctuation patterns (right column) for an offxviii 
critical shallow double quench case (A to B) at the following dimensionless times respectively: $\quad t^{*}=1.2286 \times 10^{-3}, \quad t^{*}=1.2567 \times 10^{-3} \quad$ and $t^{*}=1.2840 \times 10^{-3}$

Figure 5.6(a): Dimensionless 2D contour spatial concentration profiles for $c^{*}\left(x^{*}, y^{*}\right)$ (left column) and 3D concentration fluctuation patterns (right column) for an offcritical deep double quench case within NG region (A to C) at the following dimensionless times respectively: $t^{*}=1.2179 \times 10^{-3}, t^{*}=1.2240 \times 10^{-3}$ and $t^{*}=1.2254 \times 10^{-3}$

Figure 5.6(b): Dimensionless 2D contour spatial concentration profiles for $c^{*}\left(x^{*}, y^{*}\right)$ (left column) and 3D concentration fluctuation patterns (right column) for an offcritical deep double quench case within NG region (A to C) at the following dimensionless times respectively: $t^{*}=1.2259 \times 10^{-3}, t^{*}=1.2263 \times 10^{-3}$ and $t^{*}=1.2266 \times 10^{-3}$

Figure 5.7: Typical time evolution of the one-dimensional cross-section through one of the droplets depicting the formation and dissolution of a cavity 139

Figure 5.8: Dimensionless 2D contour spatial concentration profiles for $c^{*}\left(x^{*}, y^{*}\right)$ (left column) and 3D concentration fluctuation patterns (right column) for an offcritical deep double quench case within SD region for polymer phase and NG 
region for solvent phase ( $A$ to $D$ ) at the following dimensionless times respectively: $\quad t^{*}=1.2349 \times 10^{-3}, \quad t^{*}=1.2405 \times 10^{-3} \quad$ and

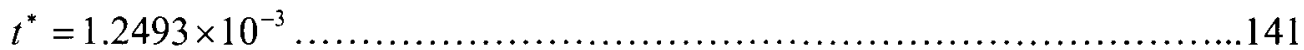

Figure 5.9: Schematic cross section of concentration profile corresponding to cavity formation in a droplet.

Figure 5.10: Schematic cross section of concentration profile representing the cavity deformation in a droplet. 143

Figure 5.11: 3D concentration fluctuation patterns $c^{*}\left(x^{*}, y^{*}\right)$ for an off-critical deep double quench case within $\mathrm{NG}$ region (A to $\mathrm{C}$ ) at $t^{*}=1.2266 \times 10^{-3}$ 145

Figure 5.12: The comparison between the simulated morphology and the experimental results $[23,136,138]$ .146

Figure 5.13: Typical light scattering profile showing the evolution of phase separation by $\mathrm{SD}$ in the early to the beginning of the intermediate stages. Each profile represents the intensity at a certain time $t$. The increase in time is indicated by the arrow in the up direction. The scattering intensity is increasing with time. 
Figure 5.14: The evolution of the dimensionless structure factor for off-critical shallow double quench (A to B) at different dimensionless times within NG region 151

Figure 5.15: The evolution of the dimensionless structure factor for off-critical deep double quench (A to C) at different dimensionless times within NG region. The second broad peak is caused by the cavity formation inside the solvent droplets. 153

Figure 5.16: The evolution of the dimensionless structure factor for off-critical deep double quench (A to D) at different dimensionless times within NG (solvent) and SD (polymer) region. The second broad peak is caused by the cavity formation inside the solvent droplets. The value of dimensionless structure factor tends to increase exponentially with time in the early stages of phases separation by SD

Figure 5.17: The evolution of the dimensionless maximum structure factor versus dimensionless time for shallow (A to B), deep (A to C) and deeper (A to D) double quench cases respectively. The natural logarithm of the maximum structure factor increases linearly (non-exponentially) with time for A to B and C quenches which is considered to be typical of NG mechanism while, it increases exponentially with time for A to D case which is the characteristic of 
SD mechanism. Transition time (downward arrow) is at

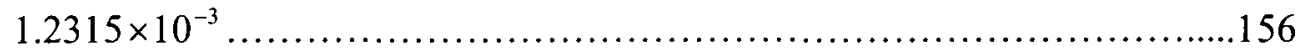

Figure 5.18: Dimensionless $2 \mathrm{D}$ contour spatial concentration profiles for $c^{*}\left(x^{*}, y^{*}\right)$ (left column) and 3D concentration fluctuation patterns (right column) for an offcritical deep double quench case (A to D) at the following dimensionless times respectively: $t^{*}=1.0850 \times 10^{-3}, t^{*}=1.0875 \times 10^{-3}$ and $t^{*}=1.0950 \times 10^{-3}$ where

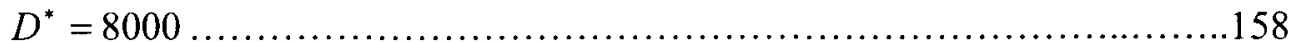




\section{List of Tables}

Table 5.1: Parameter values used for the shallow and deep double quench phase separation within NG and SD regions in TIPS method. 134

Table 5.2: Dimensionless parameters used in the simulation for the analysis of structure factor evolution (fist three cases where $D^{*}=5000$ ) and the investigation of thermal diffusion coefficient effect on TIPS in binary polymer solutions with $D^{*}=8000$ 150

A: Numerical parameter values featuring different system models with different average concentrations $\left(c_{\circ}\right)$, mesh sizes, quench depths (temperatures), diffusions and transition times respectively.

B: Parameter values. .178 


\title{
Nomenclature
}

\author{
Symbols Description \\ $A(\mathbf{k} ; t) \quad$ Magnitude of the Fourier Transform of concentration fluctuations \\ $c$ \\ Solvent concentration (volume fraction) \\ $c_{c} \quad$ Critical solvent concentration (volume fraction) \\ $c_{i} \quad$ Concentration of $i$ th component in the solution (volume fraction) \\ $c_{0} \quad$ Initial average solvent concentration (volume fraction) \\ $c_{u} \quad$ Upper equilibrium value of solvent concentration (volume fraction) \\ $c_{1} \quad$ Lower equilibrium value of solvent concentration (volume fraction) \\ $c_{\alpha} \quad$ Solvent concentration in $\alpha$ phase of polymer solution (volume fraction) \\ $c_{\beta} \quad$ Solvent concentration in $\beta$ phase of polymer solution (volume fraction) \\ $D \quad$ Mutual diffusion coefficient $\left(\mathrm{m}^{2} \mathrm{~s}^{-1}\right)$ \\ $D_{1} \quad$ Self-diffusion coefficient of solvent $\left(\mathrm{m}^{2} \mathrm{~s}^{-1}\right)$ \\ $D_{2} \quad$ Self-diffusion coefficient of solute (polymer) $\left(\mathrm{m}^{2} \mathrm{~s}^{-1}\right)$ \\ $f(c) \quad$ Flory-Huggins free energy of mixing $\left(\mathrm{J} \mathrm{or} \mathrm{kg} \mathrm{m}^{2} \mathrm{~s}^{-2}\right)$ \\ $F \quad$ Total free energy of mixing $\left(\mathrm{J} \mathrm{or} \mathrm{kg} \mathrm{m}^{2} \mathrm{~s}^{-2}\right)$ \\ $F_{i} \quad$ Weighted residual vector, $i=1,2,3, \ldots N$ \\ $G_{1} \quad$ Gibbs free energy of component $1\left(\mathrm{~J}\right.$ or $\left.\mathrm{kg} \mathrm{m}^{2} \mathrm{~s}^{-2}\right)$




\begin{tabular}{|c|c|}
\hline$G_{2}$ & Gibbs free energy of component $2\left(\mathrm{~J}\right.$ or $\left.\mathrm{kg} \mathrm{m}^{2} \mathrm{~s}^{-2}\right)$ \\
\hline$G_{12}$ & Gibbs free energy of solution ( $\left.\mathrm{J}_{\text {or }} \mathrm{kg} \mathrm{m}^{2} \mathrm{~s}^{-2}\right)$ \\
\hline $\mathbf{J}$ & Interdiffusional flux $\left(\mathrm{mol} \mathrm{m}^{-2} \mathrm{~s}^{-1}\right)$ \\
\hline$J_{i j}$ & Jacobian matrix, $i, j=1,2,3, \ldots N$ \\
\hline $\mathbf{k}$ & Wave vector \\
\hline$k_{B}$ & Boltzmann's constant $\left(\mathrm{J} \mathrm{K}^{-1}\right)$ \\
\hline$M$ & Mobility $\left(\mathrm{m}^{5} \mathrm{~J}^{-1} \mathrm{~s}^{-1}\right)$ \\
\hline$M_{1}$ & Self mobility of component $1\left(\mathrm{~m}^{5} \mathrm{~J}^{-1} \mathrm{~s}^{-1}\right)$ \\
\hline$M_{2}$ & Self mobility of component $2\left(\mathrm{~m}^{5} \mathrm{~J}^{-1} \mathrm{~s}^{-1}\right)$ \\
\hline $\mathbf{n}$ & Outward unit normal to a bounding surface \\
\hline$N$ & Number of nodes \\
\hline$N_{i}$ & Degree of polymerization of $i$ th component in the solution \\
\hline$N_{1}$ & Degree of polymerization of solvent \\
\hline$N_{2}$ & Degree of polymerization of solute (polymer) \\
\hline$R$ & Residual \\
\hline$R(\mathbf{k})$ & Amplification factor \\
\hline$S^{*}\left(k^{*}, t^{*}\right)$ & Dimensionless structure factor \\
\hline$S^{*}\left(k_{m}^{*}, t^{*}\right)$ & Dimensionless maximum structure factor \\
\hline$t$ & Time (s) \\
\hline$T$ & Temperature $(\mathrm{K})$ \\
\hline$T_{c}$ & Critical temperature $(\mathrm{K})$ \\
\hline
\end{tabular}




$\begin{array}{ll}T_{i} & \text { Initial temperature }(\mathrm{K}) \\ T_{f} & \text { Final temperature }(\mathrm{K}) \\ u_{a} & \text { Approximate solution } \\ u_{j} & \text { Set of unknown coefficients, } j=1,2,3, \ldots N \\ V & \text { Dotal volume of polymer solution }\left(\mathrm{m}^{3}\right) \\ x, y & \text { Change in Gibbs free energy of mixing }(\mathrm{J}) \\ \Delta G^{M} & \text { Change in enthalpy of mixing }(\mathrm{J}) \\ \Delta H^{M} & \text { Change in entropy of mixing }\left(\mathrm{J} \mathrm{K}^{-1}\right) \\ \Delta S^{M} & \end{array}$

\section{Greek Letters Description}

$\chi \quad$ Flory's interaction Parameter (dimensionless)

$\chi_{c} \quad$ Critical value of Flory's interaction parameter (dimensionless)

$\Delta \mu^{*} \quad$ Dimensionless change of the solvent chemical potential

$\lambda \quad$ Wavelength for fluctuation (angstrom)

$\Lambda \quad$ Kinetic factor (Onsager coefficient)

$\mu_{1} \quad$ Chemical potential of solvent $\left(\mathrm{J} \mathrm{mol}^{-1}\right)$

$\mu_{2} \quad$ Chemical potential of polymer solute $\left(\mathrm{J} \mathrm{mol}^{-1}\right)$

$v \quad$ Volume of a cell or segment $\left(\mathrm{m}^{3}\right)$

$\phi \quad$ Volume fractions of component 1

$\phi_{2} \quad$ Volume fractions of component 2 
Theta temperature $(\mathrm{K})$

\section{Superscripts Description}

$\begin{array}{ll}* & \text { Dimensionless Variable } \\ \dagger, \ddagger & \text { Respective phases of polymer solution }\end{array}$




\section{Chapter 1}

\section{Introduction}

\subsection{Phase Separation in Polymer Solutions}

The phenomena of phase separation have attracted a great deal of interest in recent years. Understanding the phase behavior and the phase separation dynamics of polymer solutions is important for many processes encountered in polymer synthesis, purification, processing, and modifications. Phase separation in polymer solutions is one of the most important techniques for manufacturing many functional polymeric materials that are widely used in engineering applications. Typical examples include porous synthetic membranes for separation processes and production, processing and modifications of PDLC films for electro-optical devices where the knowledge of the phase behavior and phase separation dynamics is essential to control the process and the final structures.

The kinetics of phase separation in polymer solutions that follow a transfer of the system from a thermodynamically stable to a thermodynamically metastable or unstable state is the subject of many theoretical and experimental investigations. Phase separation can be induced by changing the control parameters such as temperature, pressure or composition. When a 
homogenous mixture is brought into the heterogeneous state, the phase separation can proceed via either spinodal decomposition (SD) or nucleation and growth (NG). The phase separation pathway depends on the quench depth into the two-phase region $[1,2]$. Figure 1.1 shows a schematic phase diagram of a binary polymer solution.

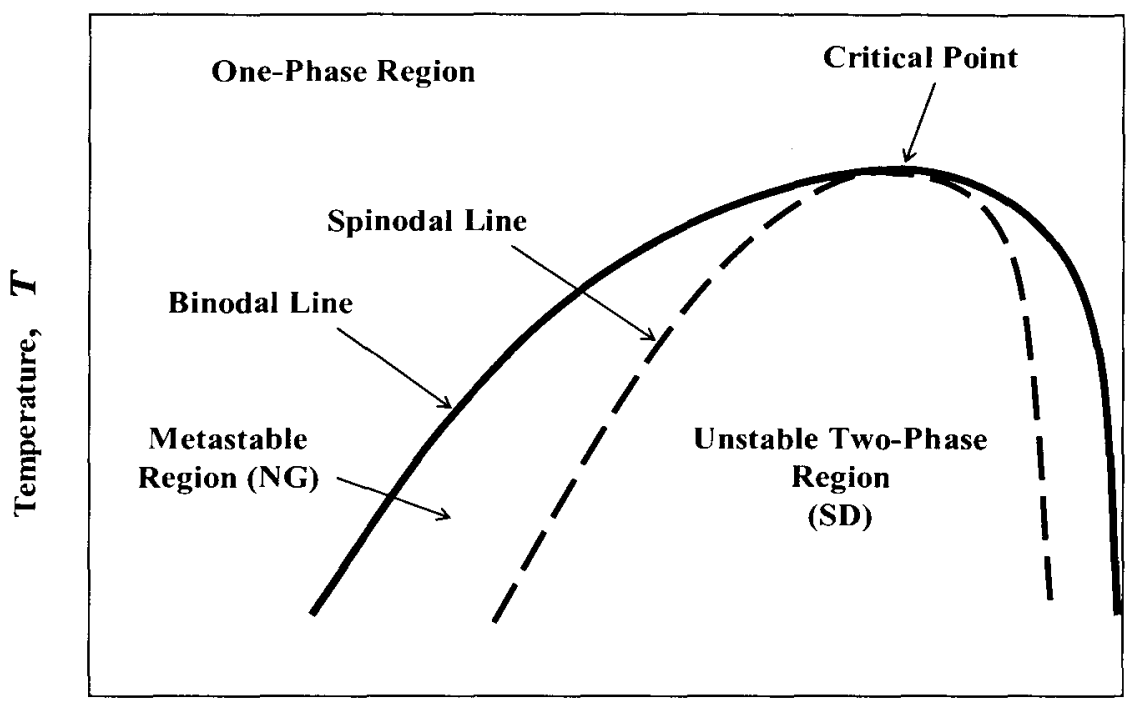

Concentration, $C$

Figure 1.1: Schematic phase diagram of a binary asymmetric polymer solution.

Spinodal decomposition occurs if the system is quenched into the thermodynamically unstable region inside the spinodal line, while nucleation and growth occurs if the system is quenched into the metastable region bounded by the binodal and spinodal lines. These two processes can result in quite different morphologies in polymer mixtures. 


\subsection{Phase Separation Mechanisms}

A common way to induce phase separation is via a temperature jump or quench into the twophase region of the phase diagram, which is known as thermally induced phase separation (TIPS). Phase separation in TIPS method often occurs via spinodal decomposition (SD) $[9,10]$. This particular process of phase separation does not require activation energy unlike NG mechanism, but proceeds spontaneously in the presence of minimal concentration fluctuations or thermal noise. The phase separation mechanism for spinodal decomposition may be classified into the following three regimes: (a) early stage, (b) intermediate stage, and (c) late stage. These stages are schematically represented in Figure 1.2. In the early stage (a), which occurs immediately after a temperature lowers from the single-phase region into the two-phase region (see Figure 1.3, D to E) at the same time, fluctuations in the average concentration $c_{\text {o }}$ lead to a change in the Gibbs free energy, $\Delta G_{M}$, that can only decrease due to the fact $\left[\partial^{2} \Delta G_{M} / \partial c^{2}\right]<0$ which will be explained in details in chapter 3 . During the early stage of $\mathrm{SD}$, the characteristic wavelength $\lambda$ of the domain size does not change with time, only the concentration difference of the polymer in polymer-rich phase and solvent-rich phase increases with time. The phase separation at this stage can be predicted by the CahnHilliard theory $[7,9,16]$. In the intermediate stage (b), the concentration difference and the characteristic wavelength both increase with time. The nonlinear effects on the time evolution of the average concentration fluctuations become increasingly important with time. The droplet size on the other hand and composition as well increase gradually with time. As a consequence, growth of the fluctuations is governed by the nonlinear time evolution equation. 

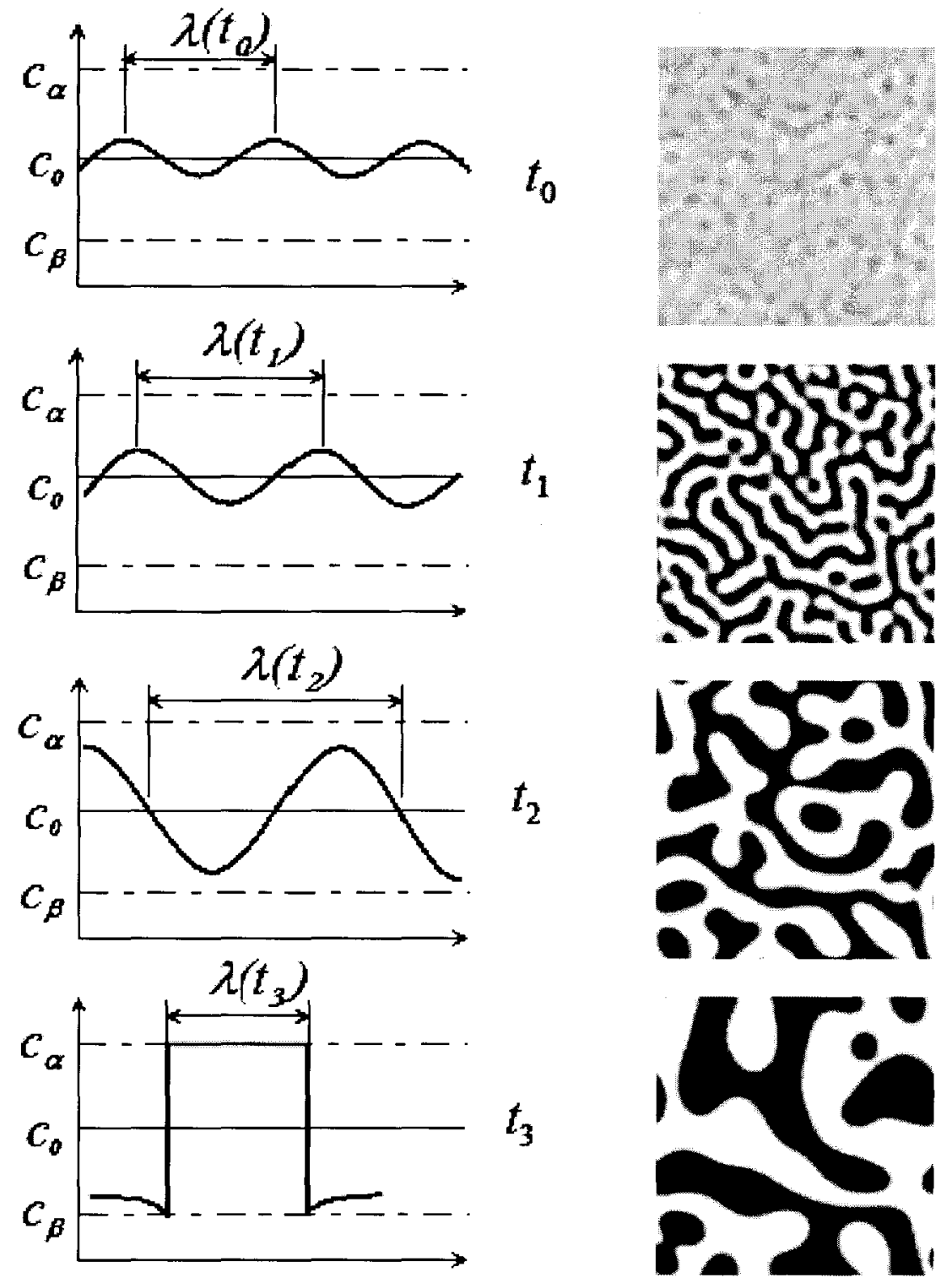

Distance, $x$

(a)

(b)

Figure 1.2: Schematic illustration of phase separation by spinodal decomposition mechanism: (a) one-dimensional evolution of concentration profiles; (b) two-dimensional picture of the resultant structure. $c_{\alpha}$ and $c_{\beta}$ are the high and low equilibrium solvent concentration on the binodal curve respectively and $t_{0}<t_{1}<t_{2}<t_{3}$ [5]. 


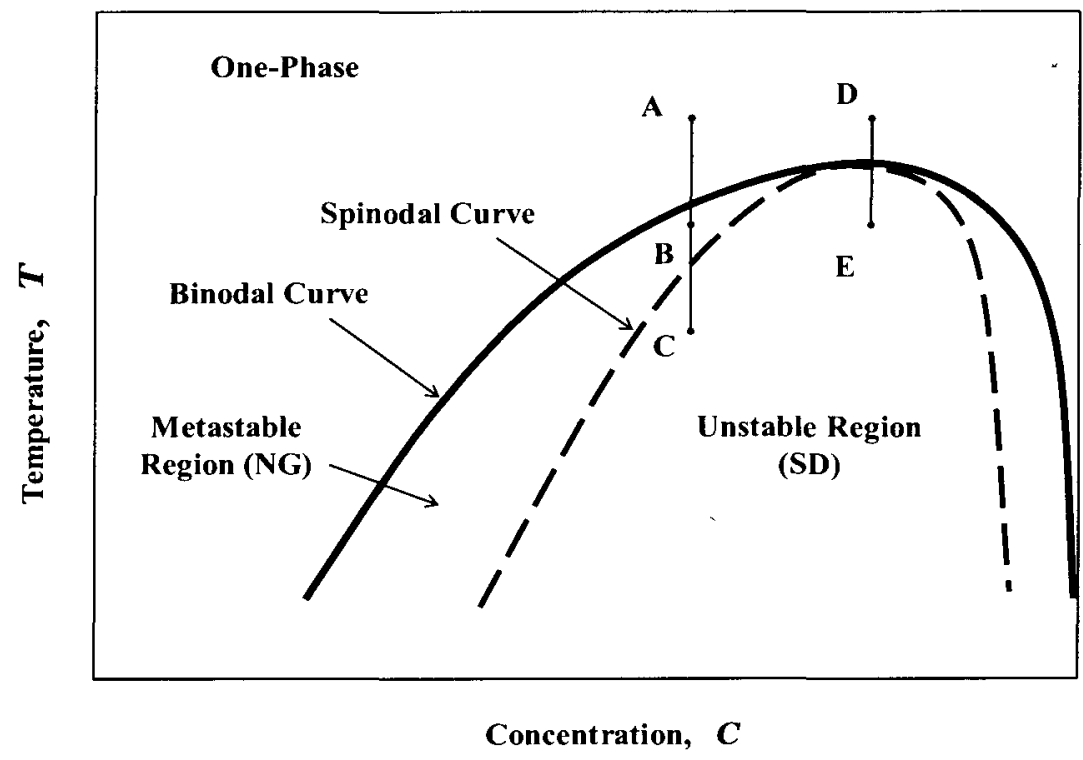

Figure 1.3: Schematic illustration of phase separation by spinodal decomposition mechanism (D to $\mathrm{E}$ or $\mathrm{A}$ to $\mathrm{C}$ ) and nucleation and growth (A to $\mathrm{B}$ ) in TIPS process, showing metastable and unstable region with binodal and spinodal lines.

In the late stage (c), the concentration fluctuation reaches the equilibrium concentration $\left(c_{\alpha}\right.$ and $c_{\beta}$ ) and the domain size is coarsening without a change in concentration, therefore phase separation is terminated by the minimization condition of the Gibbs free energy of mixing and the wavelength of the droplets is fixed by the scale of phase-separated structure. In SD, the diffusion coefficient determined by the sign of the curvature $\partial^{2} \Delta G_{M} / \partial c^{2}$ is negative. Thus, molecules diffuse up toward higher concentrations from lower concentration (uphill diffusion) causing the concentration fluctuations to grow gradually in magnitude. The mechanism of phase separation depends on the location of the solution composition relative to the binodal and spinodal compositions. 
For solutions between the binodal and spinodal compositions, small concentration fluctuations actually increase the free energy (e.g. point B of Figure 1.3), and phase separation cannot proceed until a finite nucleus with a composition close to the binodal composition spontaneously forms. With time, the initial nucleus grows while additional nuclei continue to form at random locations throughout the system. Phase separation that occurs in this way is referred to as nucleation and growth. Once these nuclei are formed, the system decomposes with a decrease in free energy, and the nuclei grow. This growth process and the corresponding phase structure are depicted in Figure 1.4. During nucleation and growth, pore growth occurs at a constant composition as material diffuses down the concentration gradient (downhill diffusion).

Phase separation is typically the result of a deep quench, and evidence suggests that spinodal decomposition is the dominant phase separation mechanism over much of the phase diagram $[11,12]$. The experimental study of spinodal decomposition in some polymer solutions is also a challenging task. The diffusion constant $D$ in these solutions is normally several orders of magnitude larger than the others, and so the time scale for the spinodal decomposition in these solutions is very small, however, near the critical point the diffusion rate is very low and the spinodal decomposition occurs on an experimentally observable time scale. Therefore, most of the experimental studies on polymer mixtures $[16,17,18]$ were done by staying close to the critical temperature. 


\subsection{Phase Separation Methods}

In recent years PDLCs have received great attention because of their leading contribution to electro-optical devices manufacturing $[1,2]$. However, these materials still present some challenging problems related to their formation and operation. Indeed, depending on fabrication parameters, e.g. time scale, the mixtures of monomers and liquid crystals can order themselves into different microstructures either by phase separation or by domain morphology. The key product application known as PDLC is obtained by phase separation process, which consists of micron-sized droplets of low weight liquid crystals dispersed in a solid polymer matrix [3]. These materials can be produced by three different commonly used techniques, namely, (i) by TIPS process $[7,11]$ from a mixture of polymers and nematic liquid crystals; (ii) by polymerization-induced phase separation (PIPS) [14,18] of a polymerizing monomer mixed with a nematic liquid crystal; (iii) by evaporation of a common solvent dissolving the liquid crystal and polymer known as SIPS [16]. As the names suggest, the phase separation is induced by the change in temperature, polymerization of the monomer and the evaporation of a common solvent respectively. Additionally, there is another method known as reaction-induced phase separation (RIPS) [19] in which phase separation occurs when the polymer is diluted in an anisotropic solvent, such as liquid crystals. TIPS process, however, is considered as a cheap, easy to handle and convenient method, while the PIPS process is useful when pre-polymer materials are miscible in low molecular weight solvents. It is interesting to note that the phase separation technique used for producing PDLC films has many advantages over other methods of manufacturing similar films [21]. 

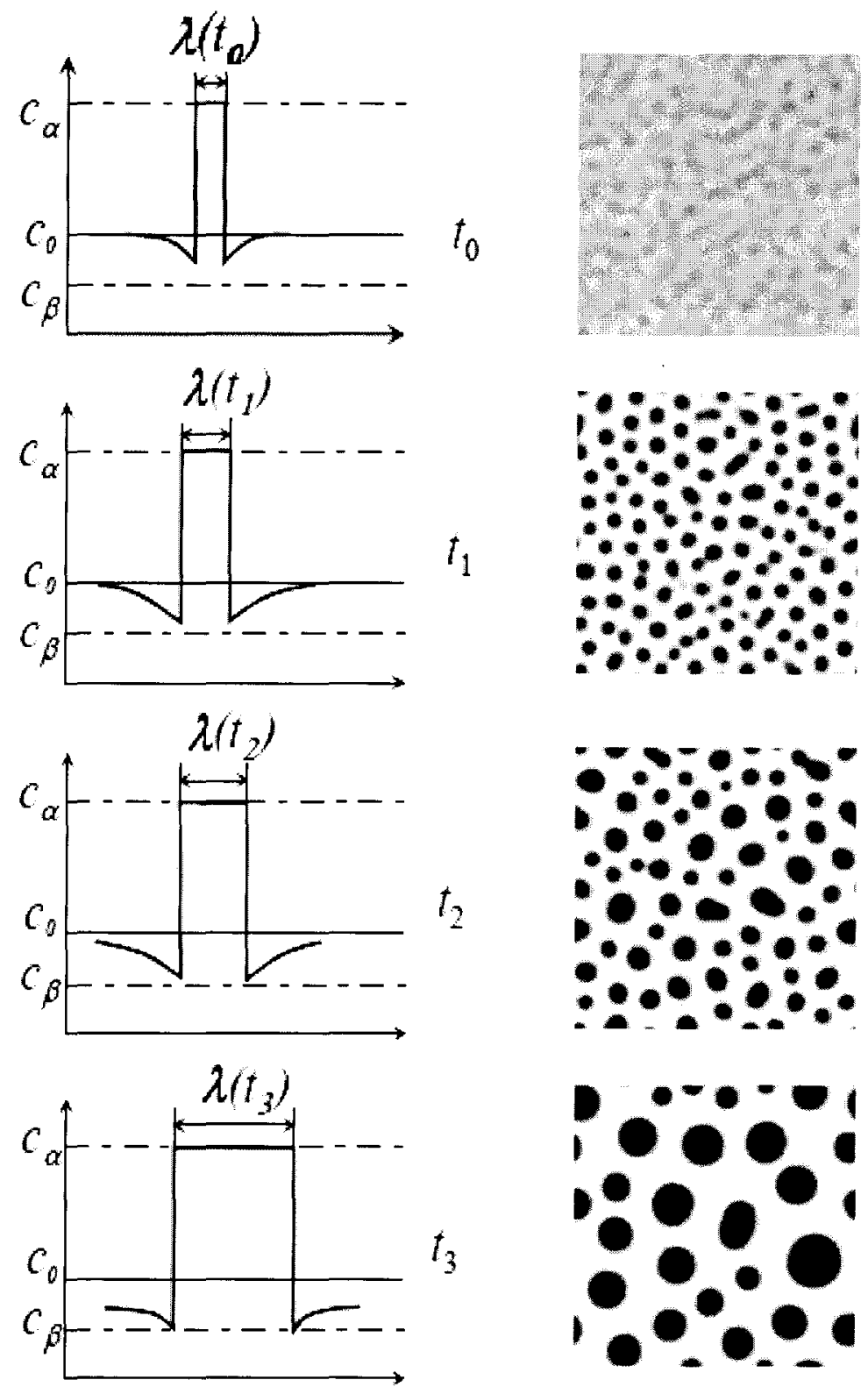

Distance, $x$

(a)

(b)

Figure 1.4: Schematic illustration of phase separation by nucleation and growth mechanism: (a) one-dimensional evolution of concentration profiles; (b) two-dimensional picture of the resultant structure. $c_{\alpha}$ and $c_{\beta}$ are the high and low equilibrium solvent concentration on the binodal curve respectively and $t_{0}<t_{1}<t_{2}<t_{3}[5]$. 
This technique, applicable to a wide range of polymers, is quite useful for controlling the size and uniformity of LC droplets. Moreover, its comparatively low production cost and simplicity are additional merits. The electro-optical properties of PDLCs can be determined by the droplet size, morphology and uniformity. The phase separation of the PDLCs mainly depends on parameters like temperature, concentration and polymerization rate (in case of PIPS), cooling rate (in case of TIPS) and evaporation rate (in case of SIPS). It must be noted that the mechanism involved in these kinds of phase separation processes is due to the competition between two contributions to the available free energy that are fully described in chapter 3. The separated state has lower entropy. The entropy is lower because the molecules of the same kind often interact between themselves more than with the molecules of other type. In the case of homogeneous state it has higher entropy. At lower temperature, energy plays a more important role than entropy, but at higher temperature the situation is the reverse. Applying these postulates, the phase transition can be predicted at some intermediate temperature, and this gives the frame of the TIPS process. In the case of PIPS, the minimum of entropy is reached when polymerizing the polymers as the bounded polymers have lower degree of freedom. The decrement of entropy is much larger in the case of homogeneous state than that of separate state.

In recent years many groups have worked on such systems similarly on different phenomenological continuous models $[20,22,24,26]$, which were utilized in the coupling of the Landau-de Gennes and Flory-Huggins free energy densities with the same microscopic techniques [23]. In this thesis we have studied the phase separation process induced by thermal double quenching during the formation of PDLC films (Figure 1.5). 

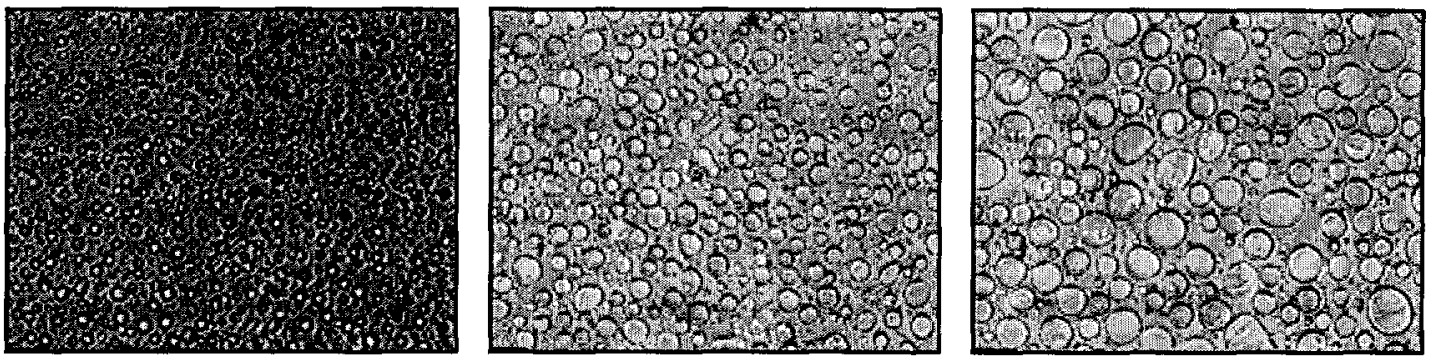

Figure 1.5: TIPS process taken at three different times for PDLC. The droplets grow in size as time passes [26].

Our main objective was the qualitative understanding of the effects of cooling rate, temperature and diffusion on the resulting droplet size and uniformity.

\subsubsection{Thermally-Induced Phase Separation Method}

Thermally-induced phase separation, or TIPS, can be used when the polymer binder has a melting temperature below its decomposition temperature. In this method, a homogeneous mixture of solvent and a melted polymer is formed. The solution is cooled at a specific rate to induce phase separation. Solvent droplets begin to form as the polymer solidifies. The droplets continue to grow until the glass transition temperature of the polymer is crossed. Droplet size is affected the most by the cooling rate of the polymer melt/solvent solution. Fast cooling rates tend to produce small droplets because there is not sufficient time for large particles to form. Therefore, droplet size and cooling rate are related inversely. Figure 1.5 shows the snapshots of TIPS process taken at three different times. Note that the droplets grow in size as time passes. 
The phase diagram for the TIPS process is a plot of concentration as a function of temperature (Figure 1.6). In the TIPS process, a single-phase mixture is prepared at a certain temperature. When the mixture is thrust into the unstable or metastable region due to temperature variation, usually one component separates from the other. Figure 1.6 shows schematically a typical phase diagram for a binary asymmetric polymer solution with an upper critical solution temperature (UCST). The dashed (dotted) curve represents the binodal (spinodal) line. The area between binodal and spinodal curves is called the metastable region, where phase separation occurs by nucleation and growth (route A). At the beginning, a polymer solution in a homogeneous phase is formed at an initial temperature and some average concentration. When the solution is cooled to a lower temperature, phase separation takes place. When the polymer solidifies, the phase separation is terminated. Two types of morphology can be obtained in the unstable region according to the average concentration. For an off-critical quench (route $\mathrm{B}$ or D) where the average concentration is not the same as critical concentration, $c_{c}$, the droplet-type morphology forms. In route B the white droplets belong to solvent phase dispersed in the matrix of polymer (solvent-rich), while in D, dark droplets are representing polymer phase dispersed inside the continuous solvent phase. Performing a critical quench (route $\mathrm{C}$ ) where the average concentration is the same as critical concentration will result in the interconnected structure. Besides the morphology, the droplet size in the TIPS process can also be controlled by the rate of cooling. In addition, there are other factors affecting the droplet sizes, such as the rate of diffusion, viscosity, and chemical potential of both components. Although the TIPS method seems simple, care must be taken to consider the process history and high temperature due to the unstable region in SD. 


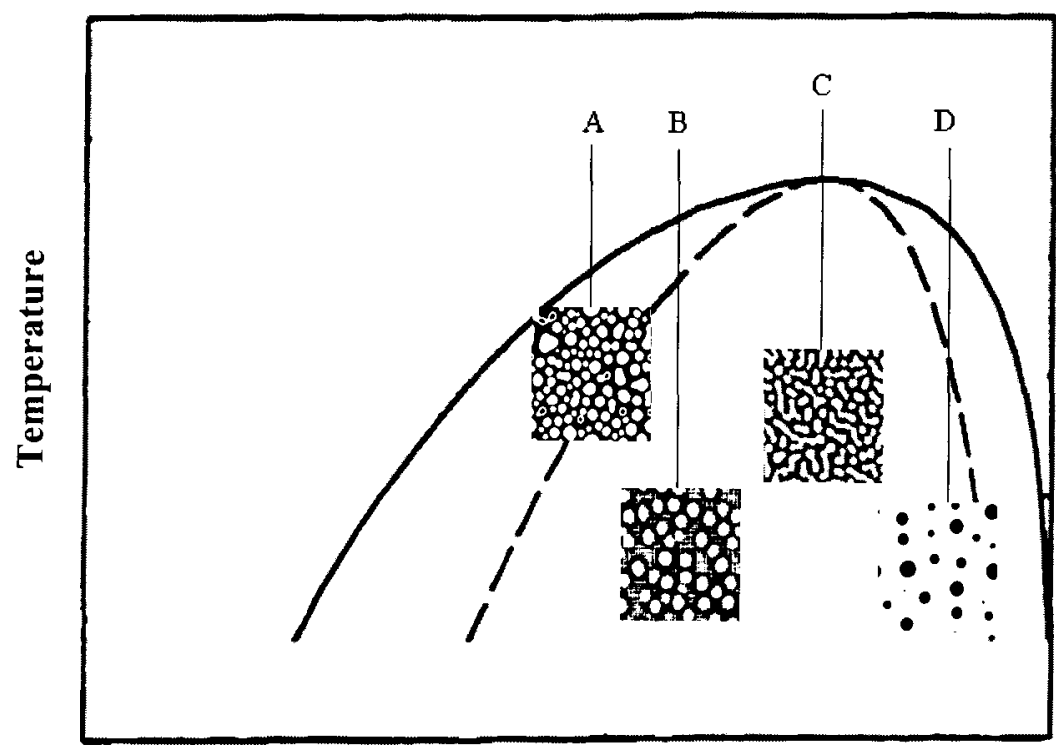

Concentration

Figure 1.6: A temperature vs. composition phase diagram of TIPS process showing the various types of quenching into spinodal region, off-critical (B and D) and critical (C). Route $\mathrm{A}$ is a quench into metastable region.

\subsubsection{Polymerization-Induced Phase Separation Method}

Polymerization-induced phase separation, or PIPS, occurs when a solvent is mixed with a solution that has not yet undergone polymerization (a prepolymer). Once a homogeneous solution is formed, the polymerization reaction is initiated. The polymerization is triggered by either heat or ultraviolet exposure in the presence of initiator or crosslinking agent, depending on the chemistry of the system. Polymerization decreases the miscibility of the two components because of the increased molecular weight of the prepolymer. At a certain 
point, phase separation occurs via either nucleation and growth mechanism, if the system is far off the critical composition, or spinodal decomposition mechanism, if the system is close to the critical composition. The morphology further evolves with polymerization until the system is "frozen" by chemical gelation. A large range of morphologies can be obtained by PIPS in a controlled manner, with the two major types being particle/matrix (or sea/island) morphology, and co-continuous morphology, as predicted by the two phase separation mechanisms. However, in reality, the situation is complicated by the competition of reaction rate and phase separation kinetics as well as the interplay of multiple sub-factors such as viscosity, temperature, non-covalent interactions, and viscoelasticity.

Another way to interpret the polymerization induced phase separation is to use the phase diagram illustrated in Figure 1.7. Here, $c_{\circ}, T_{i}$ and $N_{2}$ are the system composition (volume fraction), temperature and polymer degree of polymerization, respectively. Considering a system with an upper critical solution temperature (UCST, Figure 1.1), since most polymer mixtures exhibit UCST behavior, the system with an initial composition $c_{0}$ is initially miscible at the polymerization temperature $T_{i}$. As the polymerization proceeds, the miscible mixture becomes less stable as the phase boundary curve shifts upwards. When it reaches the curing (system) point $\left(c_{0}, T_{i}\right)$, phase separation begins. The phase diagram becomes asymmetric and the system point will either thrust into the unstable region where phase separation occurs by spinodal decomposition or metastable region where nucleation and growth is inducing the phase separation mechanism. This point is also defined as the "cloud point" (the point when phase separation begins). 


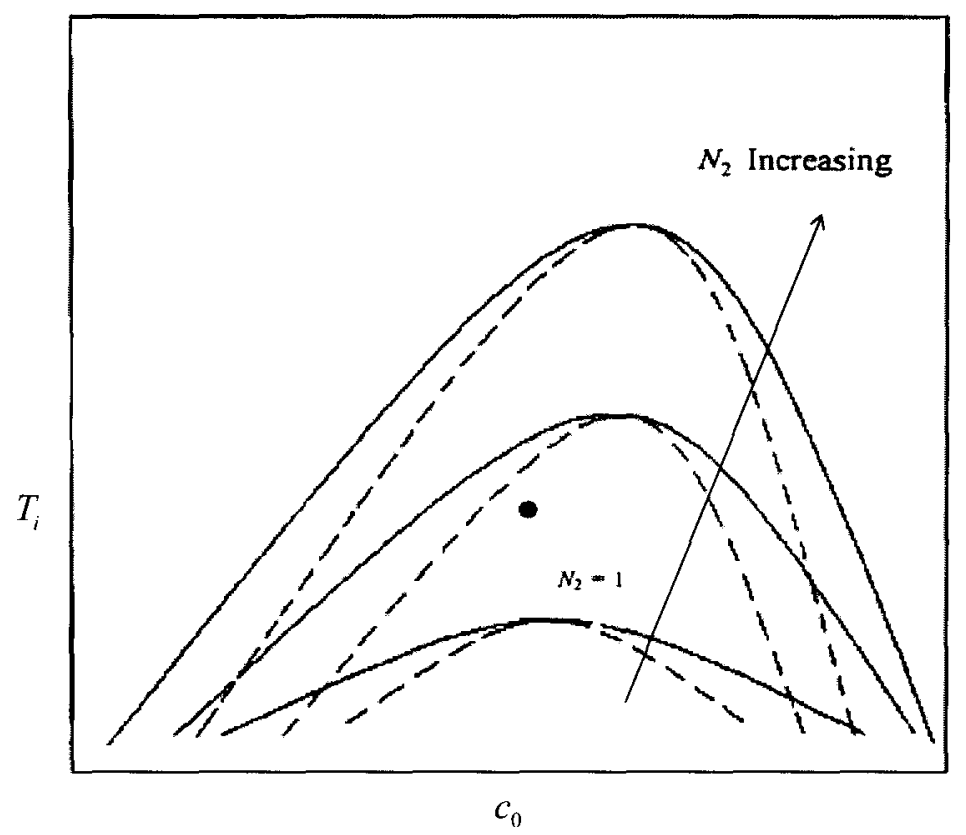

Figure 1.7: Schematic phase diagram of PIPS process. The solid (dashed) curves represent the binodal (spinodal) lines. $c_{\circ}$ and $T_{i}$ are average concentration and initial temperature respectively. $N_{2}$ is the polymer degree of polymerization which increases with polymerization. Note that the phase diagram becomes asymmetric and the system point is thrust into the unstable region where phase separation occurs by spinodal decomposition.

The two main factors that influence the size of solvent droplets in PIPS are the cure temperature and the type and proportions of materials used. The cure temperature affects the speed of the polymerization as well as the diffusion rate and solubility of the solvent in the polymer. These factors can greatly influence the size of the solvent droplets which translates into different macroscopic optical properties. 


\subsubsection{Solvent-Induced Phase Separation Method}

The third common type of phase separation is called solvent-induced phased separation, or SIPS. This process requires both the solvent and polymer to be dissolved in a solvent. The solvent is then removed (typically by evaporation) at a controlled rate to begin the phase separation. Droplets start growing as the polymer and solvent come out of solution and stop when all of the solvent has been removed. The main factor affecting droplet size in SIPS is the rate of solvent removal. Like TIPS, droplet size increases as the rate of solvent removal decreases. The SIPS method has received the least attention by researchers. The main reason for this is that some thermoplastics require strong solvents. If these solvents are not recovered, environmental problems will arise and if they are recycled, additional equipment is needed [15].

\subsubsection{Reaction-Induced Phase Separation Method}

In reaction-induced phase separation an interesting variation has been to understand phase separation kinetics when the polymer is diluted in an anisotropic solvent, such as liquid crystals. Here, there are three competing dynamics: one dominated by the transition from isotropic to nematic ordering of the liquid crystal, a second determined by the phase separation of the polymer from the liquid crystal solvent where the anisotropy of the solvent can affect solubility, and a third determined by the growing molecular weight and gelation of 
the polymer matrix [23]. PIPS, SIPS and RIPS processes are not studied in this thesis and are only briefly described.

\subsection{Double Quench TIPS Method}

Phase separation is one of the most fundamental phenomena responsible for the formation of heterogeneous structures in polymer solutions [1,2]. It is commonly observed in various kinds of polymer mixtures. Generally, the dynamics and morphology of phase separation is strongly dependent upon the quenching conditions. From this standpoint, phase-separation phenomena as already mentioned are classified into nucleation-growth (NG) type and spinodal decomposition (SD) type in the mean field picture [1,2]. Furthermore, SD-type phase separation is grouped into bicontinuous (interconnected) and droplet SD. So far, most of studies on phase separation have been focused on the ordering process accompanied by a single rapid temperature quench from a one-phase to a two-phase region [10,12]. Kinetics of phase separation induced by a single temperature quench has reasonably been understood by intensive researches [12-17]. A quench condition including the composition and temperature of a solution is one of the key factors that determine how the phase separation proceeds. There is a possibility that a variety of interesting phase-separated patterns are created by complex temperature modulations. One of such examples is periodic spinodal decomposition, which was theoretically predicted by Onuki [27] and experimentally studied by Joshua et al. [28]. In this thesis the attention is on a new type of multiple quench, namely, a double quench. 


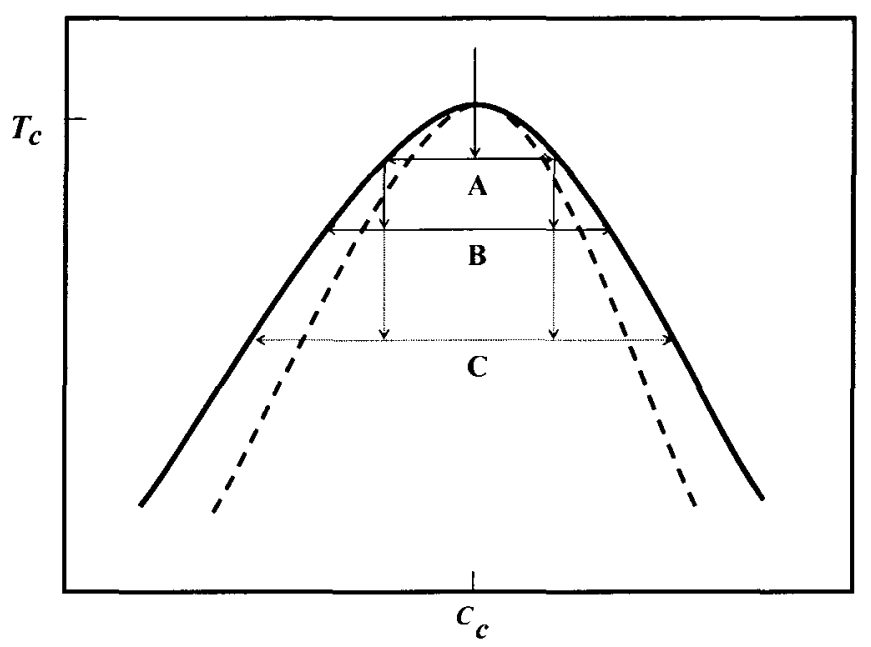

(a)

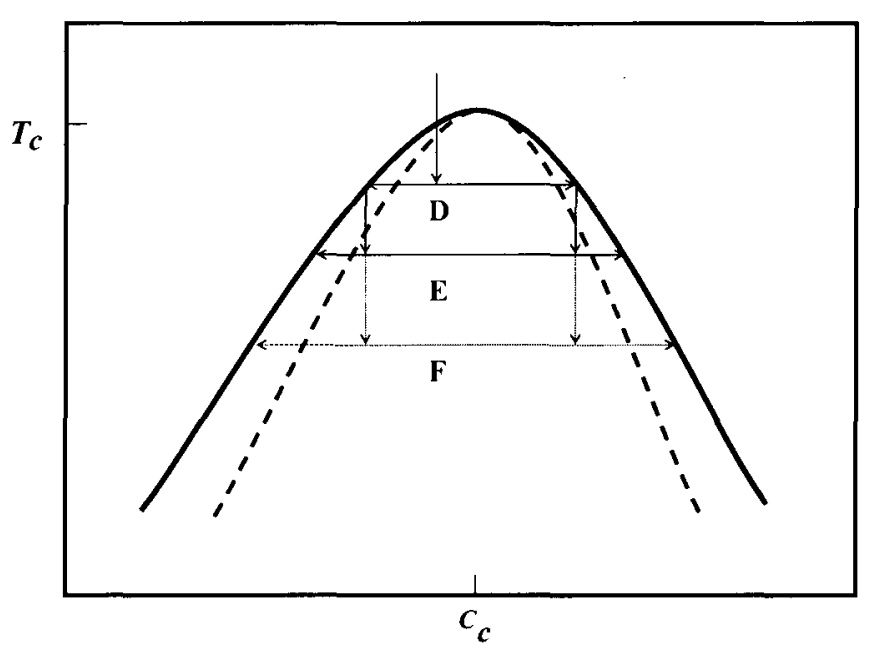

(b)

Figure 1.8: Schematic phase diagram of TIPS process. The solid (dashed) curves represent the binodal (spinodal) lines. $T_{c}$ and $c_{c}$ are critical temperature and concentration respectively. In case (a) the solution is critically quenched into $\mathrm{A}$ first and then to $\mathrm{B}$ or $\mathrm{C}$ featuring shallow and deep quenches while in case (b); the solution is quenched off-critically into $D$ first and then to $E$ and $F$ for shallow and deep quenches. 
There are so many different types of double quenches that have been investigated experimentally $[16-21,59,60]$ and numerically $[25,46,49-52$,$] . Figure 1.8$ shows two common cases of double quench studies. Case (a) is composed of two pathways; quenching from onephase to unstable region (to A), where phase separation mechanism is caused by SD and the second quench could be shallow (to B) or deep (to C) within the unstable region where phase separation would still be followed by SD. In case (b), the solution is quenched off-critically into unstable region first (to D) where phase separation is caused by SD and the second quench depending on shallow (to E) or deep mode (to F) is made into unstable region. The morphology of both cases is dissimilar and will be discussed in details in chapters 2, but in both cases, however, new droplet formation has been observed which is known as secondary droplets. These small droplets will appear inside the phase separated structure resulted from the first quench.

Although the final equilibrium state is determined solely by the final temperature, the process is strongly affected by the quench procedure. Thus, it is important to clarify how the quench history affects the kinetic pathway of phase separation. In the thesis, this problem is considered for two different double quenches, where the second quench occurs in different depths: a. shallow quench; inside the metatable region and $b$. deep quench that brings the components' concentrations down into the unstable and metastable region at the same time. The pattern evolution caused by these types of double quenches has been numerically $[49,78]$ and experimentally $[63,134-138]$ studied by a few groups. In their studies, the structural evolution has been mainly analyzed in the experimental microscopic photos and some computational studies [139] have reported the same pattern in a different approach. Instead, 
the common secondary droplet formation in double quench phase separation has been studied by several groups $[50,52,59,86-89]$. In these studies, the structural evolution has been mainly analyzed in the wave number $(q)$ space. Here we aimed at elucidating the characteristic feature of the pattern evolution induced by a double temperature quench by presenting and analyzing the two-dimensional contour morphology along with their three-dimensional graphs.

\subsection{Thesis Objectives}

The objective of this thesis is to present results from a mathematical study of the phase separation phenomena for a model of polymer solution when it undergoes a two-step quench within the metastable region of its phase diagram using the nonlinear Cahn-Hilliard equation and Flory-Huggins free energy equation. The model polymer solution is a low molecular weight solvent and a polymer with a degree of polymerization of only ten. The objectives are presented in details as following:

1. To develop and implement a numerical method that solves a model composed of the nonlinear Cahn-Hilliard and Flory-Huggins theories with boundary conditions and thermal concentration fluctuations in order to study the single quench and double quench TIPS method of phase separation in an asymmetrical polymer solution.

2. To develop, implement, solve, and validate a model that describes the pattern formation in one-step and two-step thermal quenching to compare the results with the experimental work 
that has been done on the phase separation and droplet formation phenomena for the TIPS method.

3. To develop, implement, solve, and validate a model that presents two-dimensional simulation of the phase separation and droplet formation phenomena for the TIPS method. The model will incorporate the Cahn-Hilliard and Flory-Huggins theories.

4. To develop, implement, solve, and validate a model that presents two-dimensional simulation of single quench and double quench to verify that the model will show the same known trends associated with spinodal decomposition and nucleation and growth using TIPS method.

5. To develop, implement, solve, and validate a model that presents two-dimensional simulation for a double quench in order to better understand the mechanism of secondary phase separation by comparison to the published experimental work on double quench TIPS method and clarify the characteristic feature of the pattern evolution induced by a double temperature quench by combining both morphology and wave number analyses.

\subsection{Thesis Methodology and Approach}

Computer simulations also provide a route from the microscopic detail (atomic masses, atomic charges, etc) to the macroscopic details (equations of state, material properties, etc). 
They also allow the straight-forward explanation of details at the microscopic scale, which can be hard (if not impossible) to investigate from experiment. For instance, the structure of a fluid is easily found from simulation but with somewhat more difficulty from the experiment. In addition, it also provides a route to determine properties in situations out of the reach of experiment, such as high pressures or temperatures [77]. However, in all cases, predictions of properties are dependent on a good model of the interactions between particles and efficient computer simulation methods.

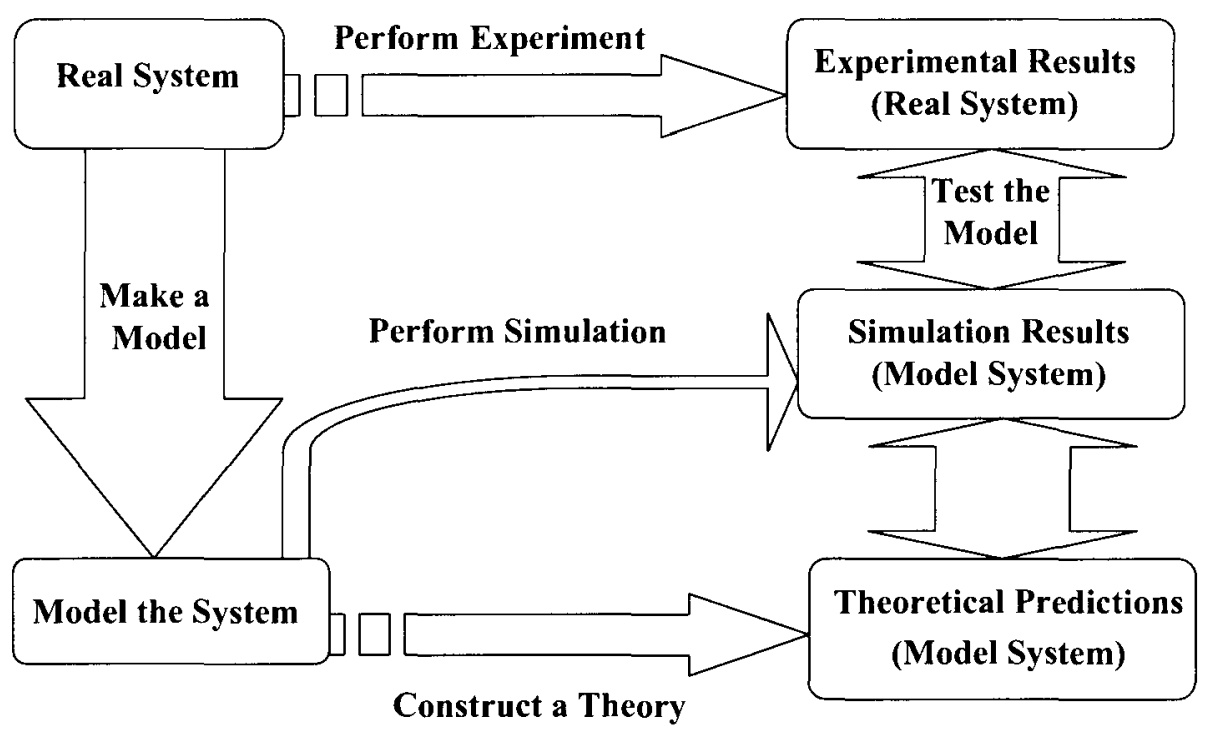

Figure 1.9: The theory, computer simulation and experiment [77].

This thesis has focused on the computational modeling and mathematical simulation of phase separation in polymer solutions undergoing spinodal decomposition and nucleation and growth mechanism for verifying the phase separation phenomenon in polymer solutions 
under double quench. The objectives of the thesis in fulfilling the above mentioned parameters is shown in Figure 1.10 and described below. The flowchart is designed for better understanding the quench process of polymer solutions with the help of mathematical modeling.

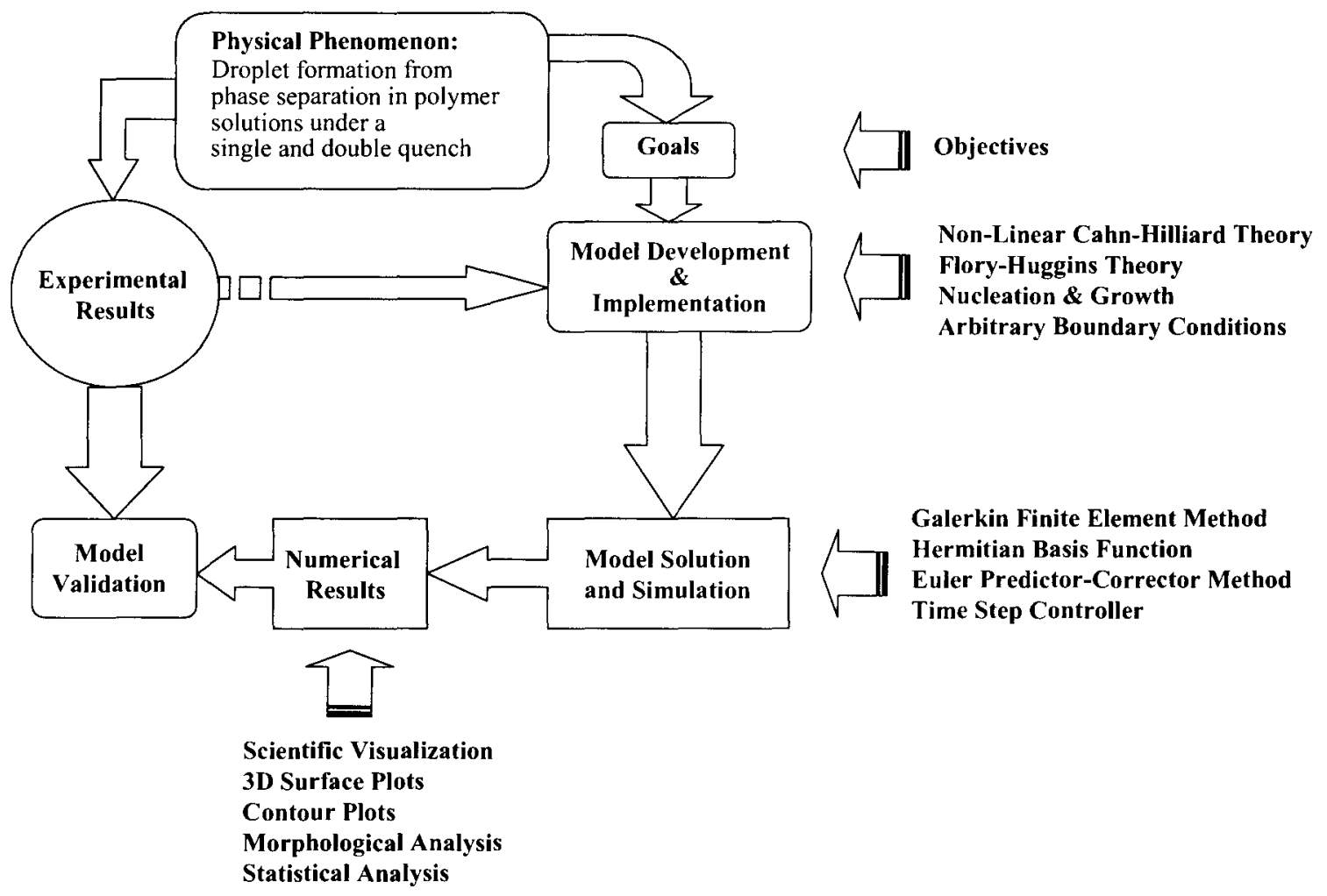

Figure 1.10: Computational modeling flowchart of the thesis objectives.

The flowsheet specifies that the prime step in the simulation of a physical experience is suggesting objectives. A model can then be developed and implemented based on the objectives, existing theories, and experimental results. This model is then solved using the 
proper computational methods and computer hardware. Lastly, the numerical results obtained from the simulations are processed so that they can be validated with on hand experimental data.

\subsection{Thesis organization}

This thesis contains seven chapters that are organized in the following way:

Chapter 1: This chapter is an introduction and overview of phase separation phenomenon with its different methods and mechanisms. The focus of this thesis is on thermally induced phase separation for a single (one-step) and double (two-step) quench. The thesis objectives, the methodology approach and the thesis organization are also covered.

Chapter 2: This chapter will present a literature review about the history of phase separation phenomenon mechanisms and the published findings of TIPS method detailed in both single and double quenching. It will furthermore outline the new experimental and numerical work and researches that have been done yet.

Chapter 3: This chapter discusses the theoretical background of phase separation along with its governing thermodynamic equations caused by spinodal decomposition mechanism. Cahn-Hilliard theory and Flory-Huggins free energy is introduced and applied. 
Chapter 4: This chapter will deal with the model development technique and the method of applied solution. Initial and boundary conditions are also presented. The model is then derived in two dimensions.

Chapter 5: In this chapter, the two-dimensional results for double TIPS quench method are discussed and analyzed. Off-critical quench cases are simulated. Different quench temperature and diffusion coefficients are examined. The concentration profiles are presented in three-dimensional graphs and two-dimensional contour plots. The simulation is also validated by comparison to the similar experimental or different numerical work.

Chapter 6: This chapter will discuss the conclusions on all numerical findings on double quenching. 


\section{Chapter 2}

\section{Literature Review}

This chapter introduces a brief history of PDLC films as a result of phase separation method. Various simulation approaches applied to PDLC systems by different groups are reviewed here. In addition some new findings are also extensively described. The theory of spinodal decomposition is then explained. The mechanism of SD to form binary polymer solutions due to the temperature variations is summarized. Two types of phase separation mechanism in TIPS method have been discussed in details for binary polymer solutions with any related numerical or experimental work that has been done so far. The new type of core-shell morphology by double quenching is introduced as well.

\subsection{Polymer Dispersed Liquid Crystal (PDLC)}

As telecommunication and computing have advanced the need for new optically active materials remains an important concern, especially for options that minimize power consumption as well as weight. Liquid crystals (LC's) have offered a successful option to this dilemma in recent years. The liquid crystal display or LCD, for example, has become the 
leading material in the manufacture of such mobile devices as personal digital assistants, notebook computers, and flat-panel televisions. The use of LC's, however, is not limited to use in displays. In the past decade one class of LC-polymer composites has shown much promise in expanding the role of these small molecules to include variable light valves, privacy windows, or even laser resonators [3,7]. These composites, called PDLCs, consist of LC droplets that are randomly dispersed in a solid polymer matrix as shown in Figure 1.5 $[3,4]$. The PDLC film is a sort of "swiss cheese" polymer with LC droplets filling in the holes. These tiny droplets (a few microns diameter for practical applications) are responsible for the unique behavior of the material. The history of PDLCs began with Hilsum et al. in 1976, when he reported glass bead dispersed in a nematic LC [38]. Not much became of these initial results until in the early to mid 1980s when Craighead reported studies on a porous matrix of esters of cellulose filled with a LC [29]. In 1985, Fergason and Drzaic reported the formation of LC-polymer composites formed from the dried mixture of LC in aqueous PVOH [35,36]. Later in 1986, Doane reported the induced phase separation of a homogeneous mixture of a LC and a prepolymer [23]. Since then, several different methods of fabrication and the design of a PDLC are under study and promise to yield new display technologies [20]. PDLCs are usually fabricated in two ways: encapsulation and phase separation. The former method was introduced by Fergason [35] and Drzaic [36] who dried a polymer solution (polyvinyl alcohol) with emulsified LC droplets. The latter method was reported by Doane et al. in 1986 [23]. It involved initiating phase separation by thermally polymerizing the polymer solution. Other phase separation methods such as evaporation of a solvent from a polymer-LC mixture (SIPS), temperature-induced phase separation (TIPS) and reaction-induced phase separation (RIPS) of a LC-polymer mixture can also be 
employed. In TIPS method; which is the focus of this thesis, the size of the LC droplets can be well controlled by the temperature. PDLC films are usually formed between two glass substrates coated with transparent electrodes such as indium tin oxide (ITO), typically separated by 5 to $10 \mu m$. A voltage can be applied across the PDLC film. As an electrically active film, a PDLC can be used as a light valve in which the polymer acts as an optically isotropic medium while the birefringent LC makes the composite optically active. The polymer matrix by its flexibility gives the PDLC mechanical strength [3,5]. Additionally, PDLC can be easily coated as a film onto a conducting substrate for the manufacture of electro-optical devices. The basic operation, as shown in Figure 2.1 , begins with the scattering or "off" state. At this point the alignment of the LC varies from droplet to droplet $[3,5]$. When a voltage is applied (the "on" state), the LCs, change their alignment to the direction of the field and the PDLC becomes transparent.

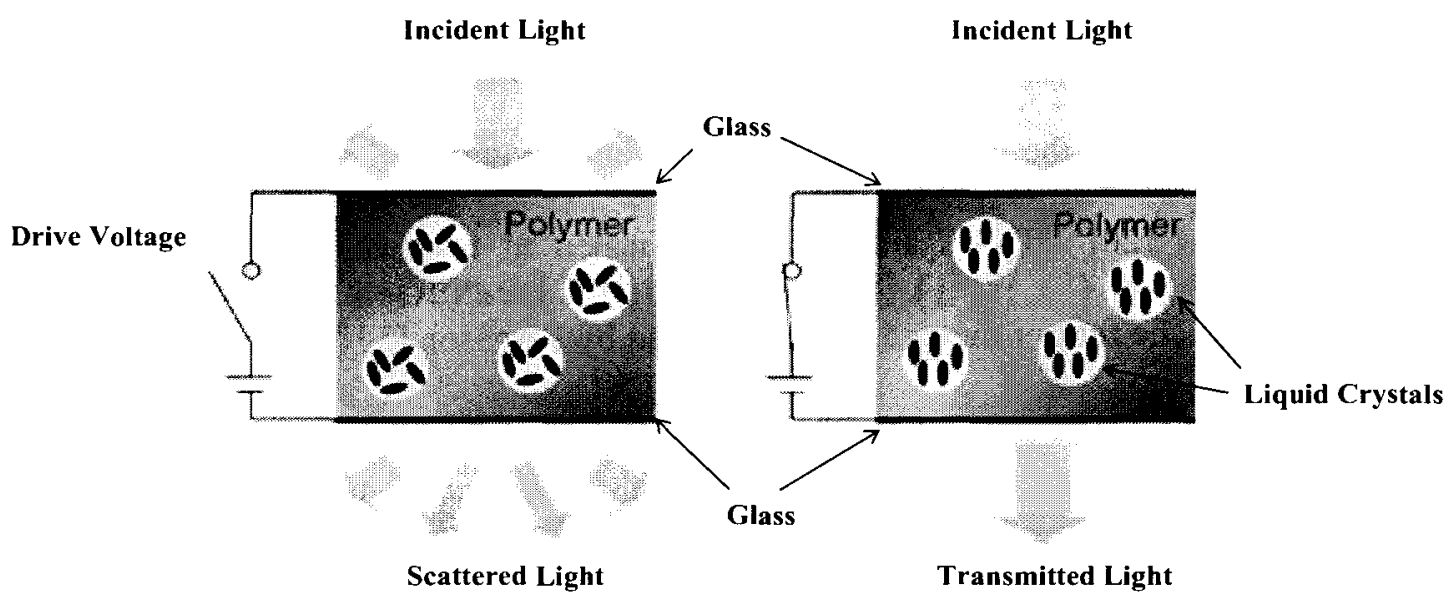

(a) Off State

(b) On State

Figure 2.1: Schematic illustration of a PDLC cell showing the scattered (transmitted) light in off-state (on-state) [3]. 


\subsection{Single Quench Studies}

In TIPS, as already discussed, phase separation in a homogeneous system of different components is brought about by the changes in temperature. When a polymer blend undergoes a quench into the spinodal region of the phase diagram random fluctuations grow rather than decay leading to two coexisting domains. To study theoretically the early and late stages of growth of the domains, Cahn-Hilliard [1] theory for the dynamics of concentration fluctuations was extended to polymeric systems by de Gennes [50] Pincus [56] and Binder [37] by combination with the well-known Flory-Huggins theory for a polymer mixture which describes the free energy of the system. In PDLC systems, this phenomenon involves the separation of a liquid crystal from an initially homogeneous solution of polymer or prepolymer. On considering PDLCs' formation and performance, many studies have been performed on the experimental side. Similarly, a lot of research on the simulation side for the PDLCs has been of the fundamental interest too. Recently, research on polymer solutions has intensified [11-19] due to the importance of these binary systems as high performance materials and the fact that final morphology controls the preferred characteristic mechanical properties of the composite. Generally, the dynamics and morphology of phase separation is strongly dependent upon the quenching conditions. From this standpoint, phase separation phenomena are classified into nucleation-growth (NG)-type and spinodal decomposition (SD)-type $[13,32]$. Furthermore, SD-type phase separation is grouped into bi-continuous and droplet SD. So far most of studies on phase separation have been focused on the ordering process accompanied by a single rapid temperature quench from a one-phase to a two-phase region [5,22]. 
Kinetics of phase separation induced by a single temperature quench has reasonably been understood by intensive researches $[37,39]$. Chan et al. $[12,15]$ studied the TIPS phenomenon via spinodal decomposition (SD) in a polymer solution under a linear concentration gradient. The model was developed using the non-linear Cahn-Hilliard theory to describe the dynamic behavior of TIPS via SD, the Flory-Huggins free energy equation for the polymer solution thermodynamics, the slow mode mobility theory and the Rouse law for the polymer diffusion process and a linear initial concentration gradient applied prior to the phase separation. As a result, the linear initial concentration gradient had an effect on the droplet distribution, the droplet size and the shape. They showed that the anisotropic morphology is due to the polymer solution undergoing SD at different rates along the sample as a result of the initial concentration gradient. This model was later successfully used $[12,14]$ to study two different types of TIPS phenomenon, namely uniform and non-uniform quench. For the uniform quench case, no spatial temperature gradient was imposed on the polymer solution, resulting in the conventional TIPS process. For the non-uniform quench case, the polymer solution sample was exposed to a linear spatial temperature gradient. The numerical results indicate that a uniform quench produces an isotropic morphology, where the droplets are uniform in size, whereas a non-uniform quench produces an anisotropic morphology, where the droplets vary continuously in size along the sample.

Gunton et al. $[20,21]$ gave an extensive review on NG and SD mechanism. His work concluded that thermally driven phase separation can result in small concentration fluctuations in the metastable region which increases the total free energy of the system. This energy is then absorbed by the binary solution. The results fit well within Flory-Huggins 
theory predictions. Adequately large but limited concentration fluctuations can decrease the total free energy of the polymer mixture which makes the solution unstable and leads to phase separation by NG mechanism. As a result, $N G$ is an activated process [10]. Extremely small concentration fluctuations in the unstable region (SD) decrease the total free energy of the system, and lead the binary solution into phase separation by SD. This type of phase separation is then a non-activated (spontaneous) process. The interconnected structure has been the solitary characteristic morphology recognized to SD for years but today experimental and numerical work has also reached different conclusions. Tanaka et al. [7] have shown that droplet-type morphology forms when performing an off-critical quench into the unstable region on a binary liquid mixture (Figure 2.2).

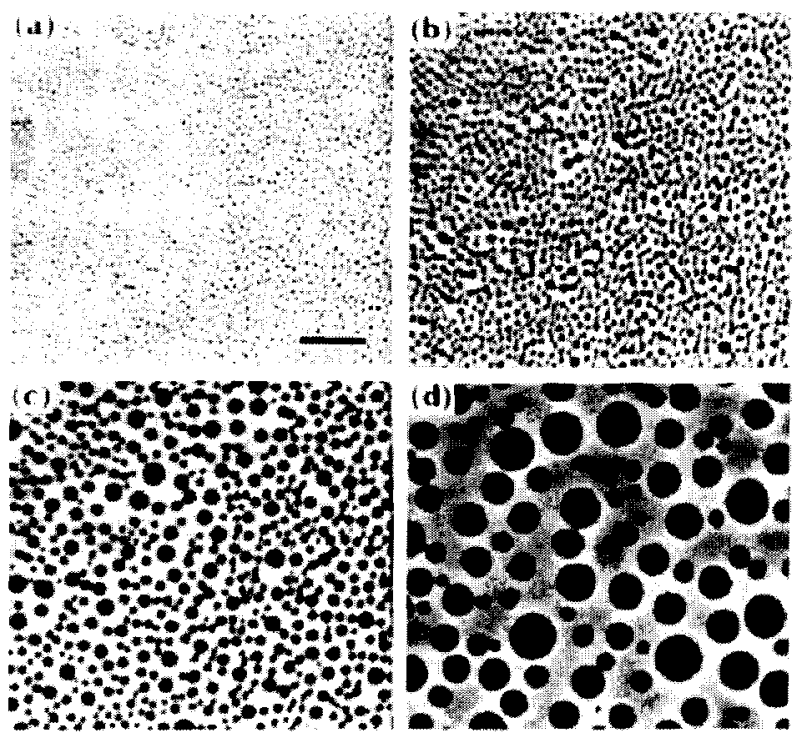

Figure 2.2: Temporal change in the patterns observed with optical microscopy during the SD-type off-critical quench in phase separation process [7]. (a) $2.5 \mathrm{~s}$, (b) $10 \mathrm{~s}$, (c) $60 \mathrm{~s}$ and (d) $240 \mathrm{~s}$ 
This morphology was later discussed by Chan et al. [30-34] numerically in details. In their work a model, composed of the nonlinear Cahn-Hilliard and Flory-Huggins theories, was used to numerically simulate the phase separation and pattern formation phenomena of oligomer and polymer solutions when quenched into the unstable region of their binary phase diagrams. The model takes into account the initial thermal concentration fluctuations. In addition, zero mass flux and natural non-periodic boundary conditions were imposed to better reflect experimental conditions. The results confirmed frequently reported experimental observations on the morphology of spinodal decomposition in binary solutions that critical quenches yield interconnected structures, and off-critical quenches give in droplet-type morphology (Figure 2.3).

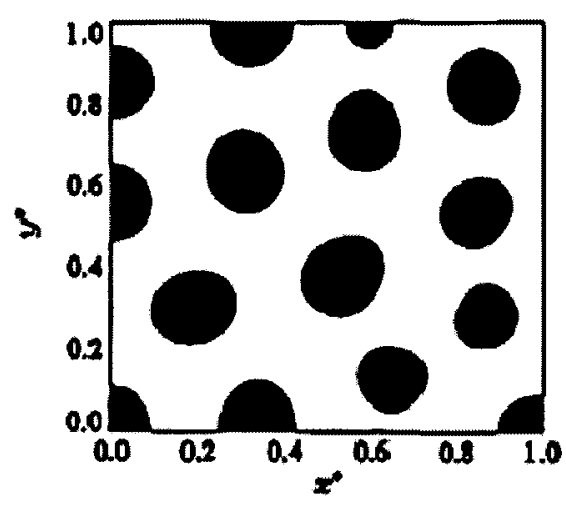

(a)

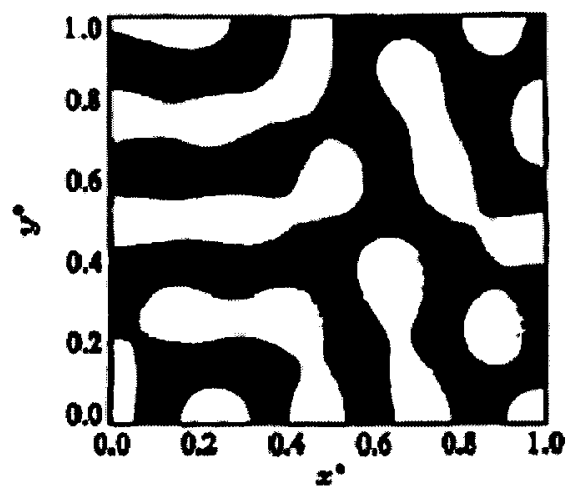

(b)

Figure 2.3: Dimensionless concentration spatial patterns formed during the SD-type phase separation phenomena for the simulation performed by Chan [30] corresponding to (a) offcritical and (b) critical quench. The black regions (white regions) in the patterns represent solvent-rich (solute-rich) regions. For an off-critical (critical) quench simulation, a droplet (interconnected) morphology structure forms [30]. 
His work could provide insights into numerical and experimental studies on the PDLC formation. In a research performed by Forrest and Heerman [68], they have demonstrated that it is possible to obtain a very good estimate for the coexistence curve for polymer blends. They claimed to have used these estimates to perform a deep quench into the two-phase region. The results indicate possible power law behavior of the typical domain size which forms after a quench even for films. They have also shown that the structure factor scales in the later stages for such polymer films. Matsuyama et al. recently studied the early stages of SD in various polymer systems by solving linearized time-dependent Ginzburg-Landau (TDGL) equations [47-49]. They calculated structure factors for concentration and orientation, depending on a quench from the stable phase into the unstable region. All three stages of SD mechanism have been analyzed and examined computationally and experimentally by a lot of researchers. There are a lot of articles and books written about different aspects of phase separation, SD and NG mechanism in general and PDLC formation and performance in particular [68]. However, this thesis is only limited to a numerical study of SD and NG phenomena for off critical quench conditions.

Kyu et al. [24] also observed droplets after quenching a liquid crystal-polymer solution offcritically into the unstable region. In his study, the system was investigated at various temperatures, concentrations, and degrees of polymerization. It was also shown that early stage SD droplets are not the ones usually observed during the coarsening process occurring in the late stage of SD. They furthermore reported the interconnected morphology by quenching the solution down spinodal region using critical concentration (Figure 2.4). Lee [69] extended the simulation to study the kinetics of microphase separation in a PDLC- 
forming process, and to study several features of the LCs. In his work, an equimolar mixture of monomer and LC molecules was thermalized and then polymerized at the same temperature. The end product was a gel with LC molecules that combined into droplets. A study of the phase separation in LC-polymer mixtures using computer simulations in two dimensions was performed by Motoyama et al. [99]. In this work, the domain morphology that results from the phase separation either by temperature quench or by polymerization was investigated by solving the coupled set of equations for the local volume fraction. In a temperature quench, it was found that transient concentric domains were constituted near the nucleation regime.

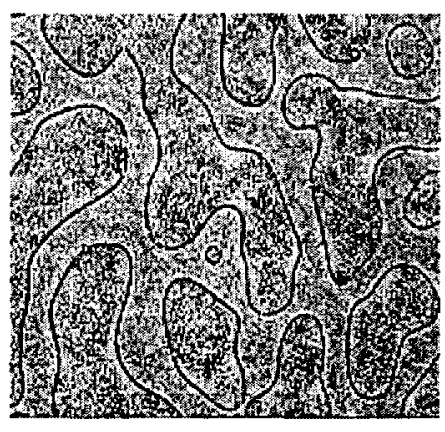

Figure 2.4: Interconnected pattern observed with optical microscopy during the SD-type critical quench in phase separation process after $360 \mathrm{~s}$ [24].

Hashimoto et al. [90,91] and Elder et al. [88] have worked on the early stages of spinodal decomposition in two dimensions. They reported an analytical numerical investigation to question the dynamics and the morphology of SD in a polymer system. According to this study, the theoretical analysis and simulation on a two-dimensional square lattice were carried out by utilizing the kinetics of the Cahn-Hilliard-Cook (CHC) equation. 
The $\mathrm{C}-\mathrm{H}-\mathrm{C}$ equation is an extension of the $\mathrm{C}-\mathrm{H}$ equation by an addition of a noise term due to the thermal motion of the atoms; the noise term appears to be important only close to the critical point [44] which will be discussed fully in chapter 3 . The theoretical and numerical results indicated interconnected morphology for SD in the early and intermediate stage of the phase separation. It should be noted that since SD is a spontaneous process, the early stages of phase separation is usually very difficult to observe experimentally, but however, the high viscosity of a polymer mixture slows down the phase separation and makes it easier to follow the early stages. On considering the importance of computer simulation, which can be considered as an alternative method besides theory and experiment, Teixeira et al. [92] developed a model of PDLC formation by TIPS. In this work, the cell dynamical systems method of Oono and Puri was employed for the spinodal decomposition in the thermoplastic-LC mixture. The numerical calculations performed on a two-dimensional system for a composition of $30 \%$ LC $70 \%$ thermoplastic reveal that the final morphology depends strongly on the quench rate. It was observed that for a much faster cooling rate, complete decomposition can be excluded, while for slower quenches, the usual LC-rich droplet pattern of constant-temperature is recovered. In this model, the PDLC is prepared by TIPS, by which the droplet formation occurs.

Copetti and Elliott [16] have solved the nonlinear $\mathrm{C}-\mathrm{H}$ equation with periodic boundary conditions into the intermediate and late stages of $\mathrm{SD}$, numerically by using the nonlinear Cahn-Hilliard $(\mathrm{C}-\mathrm{H})$ equation in two dimensions using a finite difference approximation for space and time $[55.57,58]$ and reported that droplets form for an off-critical quench and the interconnected structure forms for a critical quench. They used a fourth-order polynomial to 
approximate the free energy, which produces a symmetric phase diagram [24]. Their results are similar to the experimental observations of Kyu et al. [24] for critical quenches and Tanaka et al. [7] for off-critical quenches. Computational studies on SD by Chakrabarti [70] and Brown and Chakrabarti $[71,72]$ on the nonlinear Cahn-Hilliard-Cook equation in two dimensions $[67,68]$ and three dimensions $[31,33]$ show droplets forming for an off-critical quench that are more homogeneous in size and equally distributed than NG ones [72]. Their results however are restricted to blends of two polymers of equal polymerization degree and monomer size that makes the phase diagram symmetric. Chakrabarti used a fourth-order Ginzburg-Landau free energy, which does not consider any details of the molecules geometry in the binary solution.

\subsection{Double Quench Studies}

A review of the literature, however, shows that comparatively little work has been done on the two step mechanism of SD in polymer solutions [20,21]. Despite the frequent researches on phase separation morphology of polymer solutions induced by a single temperature quench, the dynamic behavior of a two-step quench and its following morphology is still a main unanswered dilemma. The $\mathrm{C}-\mathrm{H}$ equation $[15,20,32]$ is a linear theory that has always been applied by experimental and theoretical researchers of different fields as the preliminary spot for studying the procedure of phase separation . Nonlinear modifications of the $\mathrm{C}-\mathrm{H}$ equation have also been used to suggest new mechanisms. In this thesis however, double quench studies of phase separation have been investigated from a new pathway: quenching 
from one-phase region into the metastable region and a subsequent (shallow and deep) quench into the unstable region in order to verify the transition procedure and its core-shell (cavity) morphology resulting from the interference of nucleation and growth mechanism with spinodal decomposition mechanism.

\subsubsection{Transition from NG to SD}

It is widely known that the nucleation-growth (NG) type of phase separation occurs in the metastable region because of the existence of a barrier for nucleation, while the spinodal decomposition (SD) type of phase separation occurs in the unstable region where fluctuations can grow without any barrier. Recent theoretical studies [51-55] show that the transition from metastability (NG) to instability (SD) may be diffuse and it is sharp only in a mean field-like system. The main idea of the mean field theory (MFT), also known as self-consistent field theory; is to focus on one particle and assume that the most important contribution to the interactions of such particle with its neighboring particles is determined by the mean field due to the neighboring particles, namely; many interactions are replaced by one effective interaction. It naturally follows that if the field or particle exhibits many interactions in the original system, MFT will be more accurate for such a system. Another approximation that is made in the mean field theory is that fluctuations can be neglected. A theory of spinodal decomposition including fluctuations was formulated by Langer et al. [63]. They concluded that their theory describes reasonably well the gradual transition from spinodal decomposition to nucleation. Binder et al. [43] then discussed the kinetics of phase 
separation with emphasis on the transition between spinodal decomposition and nucleation by presenting a theory which describes a gradual transition from nucleation and growth to spinodal decomposition. They claimed the transition between nucleation and spinodal decomposition is a gradual one, although experimentally it may look rather sharp. The width of the transition regime was also estimated and interpreted.

Tanaka et al. [40] as well verified the transition in a binary mixture of oligomers of styrene and $\epsilon$-caprolactone. They studied a transition from metastability to instability by changing a quench depth systematically under an off-critical quench condition (Figure 2.5). The concentration distribution function turned out to be a good fingerprint for determining whether phase separation is nucleation-growth type or spinodal-decomposition type. They also demonstrated clear morphological and kinetic evidence of a diffuse metastable-unstable transition or crossover phenomena; theoretically predicted for the system with a finite-range interaction. They believed the broadness of the transition is closely related to the range of the bare interaction. There have been few systematic, experimental studies on the transition from metastability to instability, though there have been several interesting studies $[77,78]$ on nucleation itself. In a usual binary-liquid mixture, the low viscosity makes the phase separation so rapid that within a very short time the phase separation enters in very late stage where gravity plays a significant role. Furthermore, the phase-separation behavior is very sensitive to small temperature changes, and very delicate experiments are required. In a polymer mixture, on the other hand, the high viscosity makes the phase separation very slow. Although it is suitable for studying the initial stage of spinodal decomposition, it takes too 


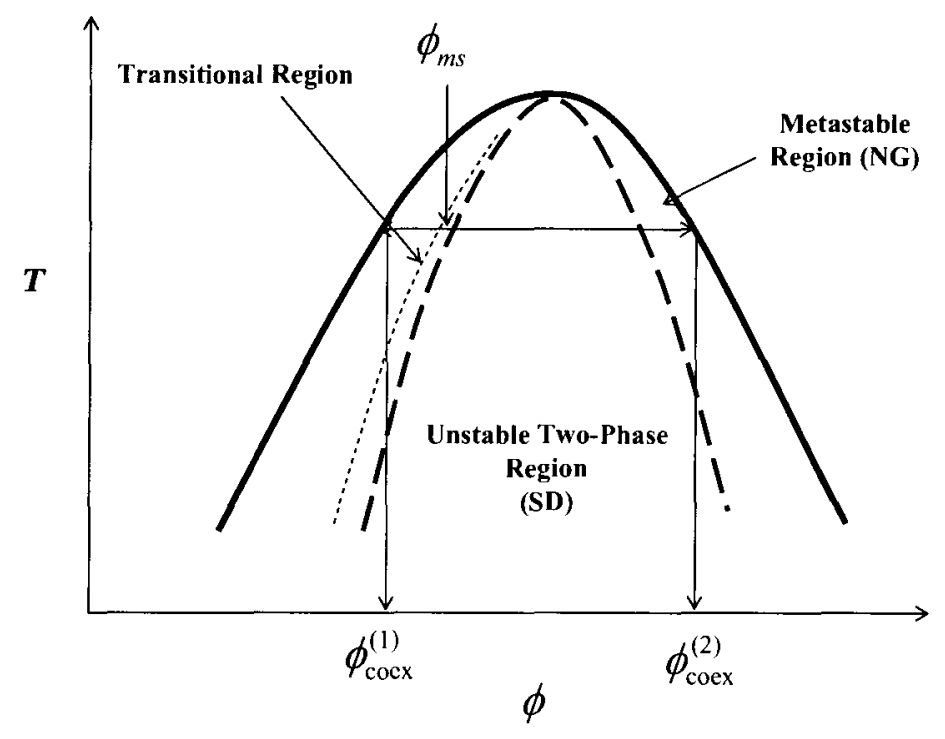

Figure 2.5: Schematic phase diagram of a binary mixture (temperature vs. concentration). The solid curve is the binodal line (the coexistence curve) and the dashed curve the classical spinodal line. The shaded area is a transitional region from metastability to instability. The broadness of the transition depends on the interaction range. The arrow indicates an offcritical quench condition. $\phi_{\mathrm{cocx}}^{(1)}$ and $\phi_{\mathrm{cocx}}^{(2)}$ are the two branches of the coexistence curve [40].

long a time to study nucleation in the metastable state or the overall phase-separation dynamics. Following Tanaka's experiment; Chakrabarti presented results from a numerical study of the Cahn-Hilliard-Cook model in two dimensions. He studied the transition from metastability to instability in his model by systematically changing the quench depth for an off-critical quench condition. He used different kinetic probes in the simulation to distinguish between two types of growth mechanisms: nucleation and spinodal decomposition. Although one can distinguish between nucleation and spinodal decomposition in some cases, the transition between these two growth processes is gradual; he believed. He did not see any evidence of a sharp transition from one to the other at the mean field spinodal line. Actually, 
the center of the diffuse transition zone that he found in the simulation was located above the mean-field spinodal line. No systematic calculation is available to predict how the kinetics of the phase-separation process should change as a function of quench depth, as one can quench the system at different points on each side of the mean-field spinodal line.

Theories that do not rely on mean-field-type approximations conclude that the transition from nucleation to spinodal decomposition is gradual. It occurs within an area above the meanfield spinodal. While simulations have supported the predictions of the non-mean-field theories, experiments that include both regimes have only been carried out with polymer blends separating into two liquid phases upon cooling [89]. This type of phase separation exhibits distinctive features. Yet, polymer blends comply with many of the mean-field assumptions. Vekilov et al. [89] monitored with optical microscopy, in real time and in real space, the generation of a dense liquid phase in high-concentration solutions of the protein solutions after temperature quenches into thermodynamically defined metastable and unstable regions. Nucleation to spinodal decomposition experiments with small molecule liquid or solid mixtures that do not fit the mean-field assumptions are difficult because of the fast nucleation rates. However, they claimed with this system, which is a poor fit to meanfield assumptions that experimental facts on the evolution of the new-phase during L-L phase separation in protein solutions show the transition between the nucleation and spinodal decomposition mechanisms of generation of the new phase is smooth and occurs over a range of driving forces. Their observations mostly agree with the predictions of the nonmean- field theories of phase transformations. 
Kiran and Liu $[93,94]$ also studied the kinetics of pressure-induced phase separation (PIPS) in solutions of polydimethylsiloxane in supercritical carbon using time and angle-resolved light scattering. Controlled pressure quench experiments were conducted at different polymer concentrations to determine both the binodal and spinodal envelopes, and the critical polymer concentration. At each concentration, they imposed a series of rapid pressure quenches with different depths of penetration into the region of immiscibility and the time evolutions of the scattered light intensities were followed to determine the pressure below which the mechanism changes from nucleation and growth to spinodal decomposition. This crossover was then identified from the characteristic fingerprint scattering patterns associated with each mechanism. The study, however, only permits the identification of the width of the metastable region.

\subsubsection{Secondary Droplets}

As mentioned before, dynamics and morphology of phase separation are strongly dependent on a quench condition, which gives a final equilibrium free energy. Thus a quench condition including the change in composition, temperature, and pressure of a mixture is one of the key factors dominating the phase separation behavior. So far most of the studies on phase separation phenomena have been limited to an ordering process accompanied by a single quench from a one-phase to a two-phase region [32,36]. For single quenches, phase separation phenomena are divided into nucleation growth (NG) and spinodal decomposition (SD), depending upon quench conditions. There is a possibility that, new types of phase 
separated patterns are caused by unusual quench conditions. From this standpoint, the problem related to fluctuations under special quench conditions has been studied by some researchers, and so far classified into the following three cases [37]: (i) A temperature or pressure quench in a stable, one-phase region [43,57]; (ii) a double quench where the system is first quenched from a one-phase to a two-phase region, and then subsequently back to the one-phase region $[61,79]$; and (iii) periodic variations of temperature which bring the system alternatively below and above the phase separation point. This latter periodic spinodal decomposition was predicted by Onuki [27] and then experimentally studied $[71,82]$.

Here we explain a different kind of double temperature quench, which consists of two cases: a. quenching from a one-phase to a two-phase region (SD) and a subsequent second quench within the two-phase region (SD), b. quenching from a one-phase region into metastable region (NG) and a subsequent second quench into the two-phase region (SD) (which is the focus of this thesis). The second quench can further be grouped into two kinds: a deeper quench and a shallower quench. These kinds of double quenches (specially the second case) are largely unexplored [73-75], although interface stability under double-quench conditions has been theoretically studied [103]. Following; we describe studies that have been done so far on the pattern evolution caused by double quenches within the two-phase region and discuss its mechanism along with original domain structure against a second quench. The two-step jump was first studied by Ohnaga and Inoue [52] who calculated the concentration profile in a one-dimensional system numerically based on the Cahn-Hilliard nonlinear diffusion equation that neglected hydrodynamic interactions (Figure 2.6). They investigated further the effect of quench depth on the change of profile induced by the shallow and deep 
quench. This was undertaken for a real polymer system. They chose the binary system polybutadiene and poly(styreneco-butadiene). The calculated phase diagram was an UCST (upper critical solution temperature) type phase diagram with the critical point being at about $400^{\circ} \mathrm{C}$. After a homogeneous mixture underwent the first temperature-jump from the singlephase region to the two-phase region of the phase diagram, the system was allowed to demix isothermally for a time, and then the demixed system underwent the second jump to a shallower or deeper quench. When the quench depth of the second jump was shallow (B to C), the concentration fluctuation decayed with time after the second jump and when the second jump was to a deeper quench (B to D), a new fluctuation of short wavelength was superimposed on the previously developed one.

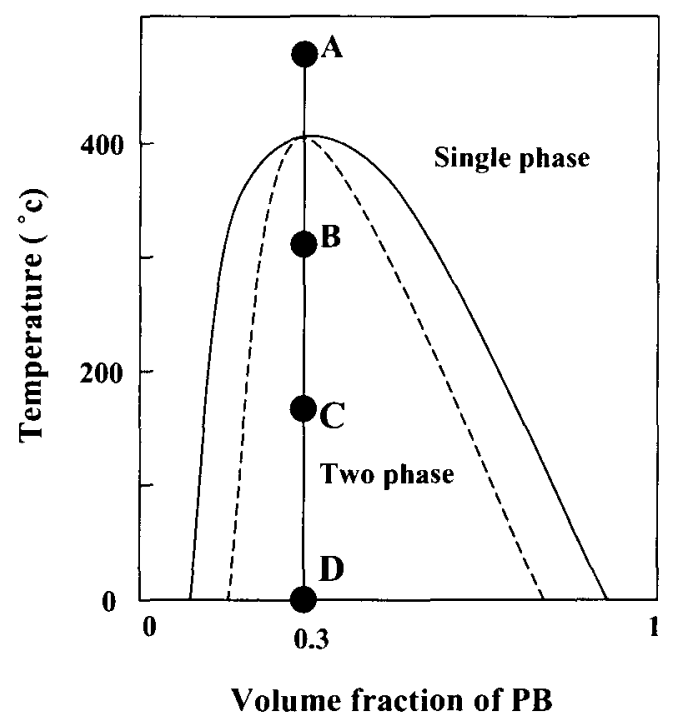

Figure 2.6: UCST-type phase diagram and two-step temperature jump: the first jump from A to B and the second from B to C (shallow) or B to D (deep): (-) binodal and (---) spinodal curves [52]. 
When the system with well-developed fluctuation was subjected to deep quenches, new short waves overlapped the old ones for a while and then the new short waves were finally absorbed into the old ones. There existed a difference in the process between the two cases however; the late-state profiles resembled each other.

Okada et al. $[59,66]$ performed the two-step temperature jump for a polymer blend and observed a new morphological structure using an electron microscope (Figure 2.7). By the second temperature jump, small droplets were emerged inside large co-continuous domains that were already formed by the first temperature jump. Inner small droplets grew in size first, but after a certain period the average sizes of droplets did not change appreciably and the number of droplets started to decrease. Independently, Tanaka [86] also studied experimentally the morphological structures formed by the two-step temperature jump with a phase-contrast microscope (Figure 2.8).

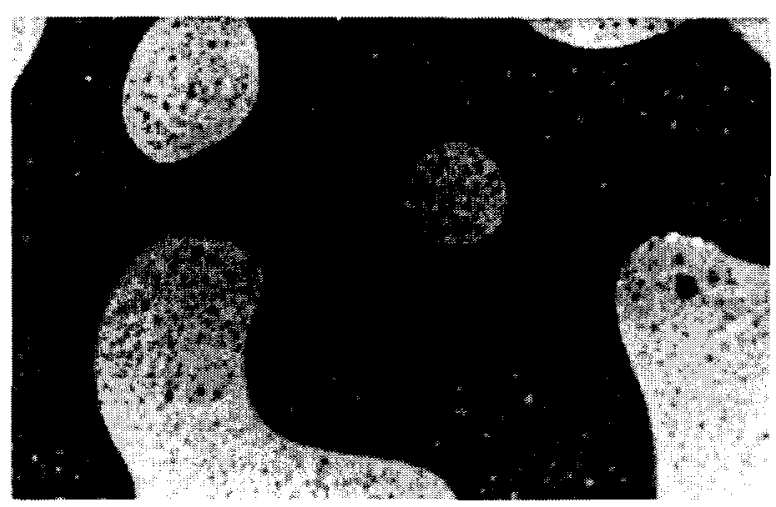

Figure 2.7: Backscattered electron image of the phase-separation structure formed in the two-step temperature jump of blend $\left(1.35 \times 10^{4} \mathrm{~s}\right.$ after the second temperature jump). White regions correspond to the poly(2-chlorostyrene)-rich phase [66]. 
He demonstrated the evolution of an unusual phase-separated pattern caused by a double quench. The first quench was from a one-phase to a two-phase region and a subsequent second quench within the two-phase region (Figure 2.9). The resulting pattern evolution strongly depends upon the type of a double-quench sequence. By the second temperature jump, small droplets were emerged inside large co-continuous domains that were already formed by the first temperature jump. Tanaka et al. [87] also found by numerical simulations that spinodal decomposition of fluid mixtures is strongly dependent upon their fluidity. Thus for a high fluidity, they claimed the double phase separation to be spontaneous. They reported a systematic numerical study on how fluidity affects the phase-separation kinetics of two-dimensional incompressible fluid mixtures.

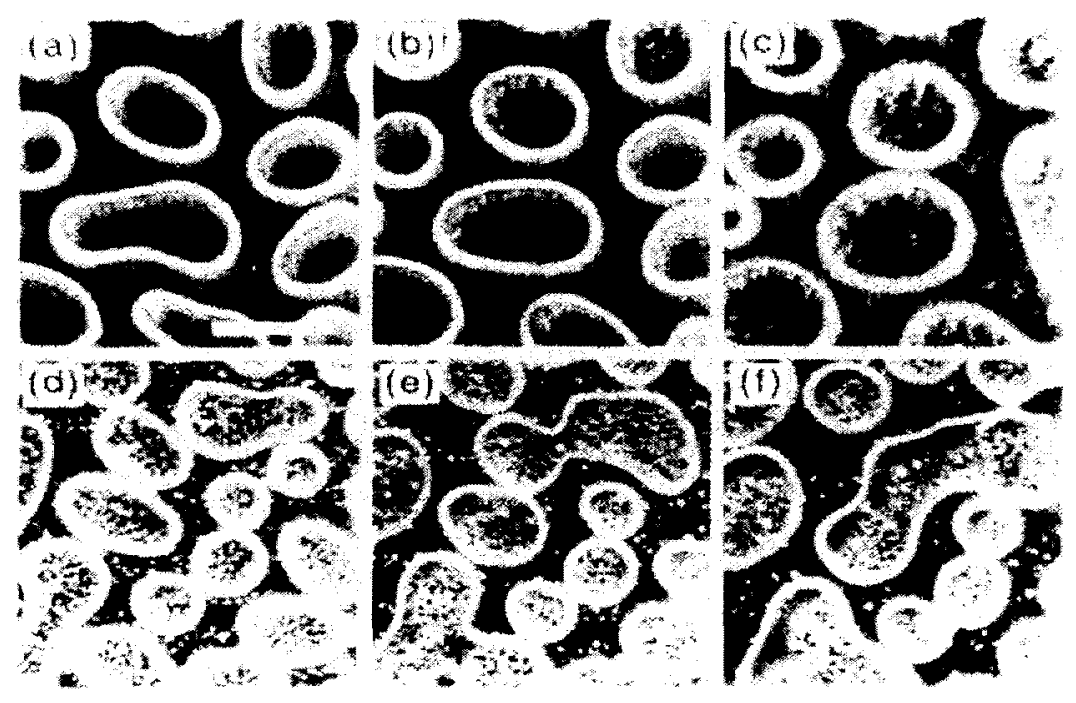

Figure 2.8: Pattern evolution caused by a double quench: $\left(6.8^{\circ} \mathrm{C}, 600 \mathrm{~s}, 21.4^{\circ} \mathrm{C}\right)\left(163.0^{\circ} \mathrm{C}\right.$, $168.0^{\circ} \mathrm{C}$ ) in a PS-PVME (50-50) mixture; (a) $60 \mathrm{~s}$, (b) $120 \mathrm{~s}$, (c) $240 \mathrm{~s}$, (d) $480 \mathrm{~s}$, (e) $720 \mathrm{~s}$, and $(\mathrm{t}) 960 \mathrm{~s}$ after the second quench [86]. 
To study phase-separation kinetics of two dimensional critical fluid mixtures, they solved the scaled kinetic equations by the Euler method under periodic boundary conditions and the incompressibility condition. Figure 2.10 represents the overall pattern evolution during bicontinuous spinodal decomposition for a symmetric composition under a high-fluidity condition [87]. It is evident that, even after the formation of a sharp interface, domains do not reach the final equilibrium composition and the temporal change in the darkness (composition) of domains can be seen. It should be noted that it has so far been widely believed that there is no change in composition after the formation of a sharp interface. This local-equilibrium assumption is the heart of the scaling concept.

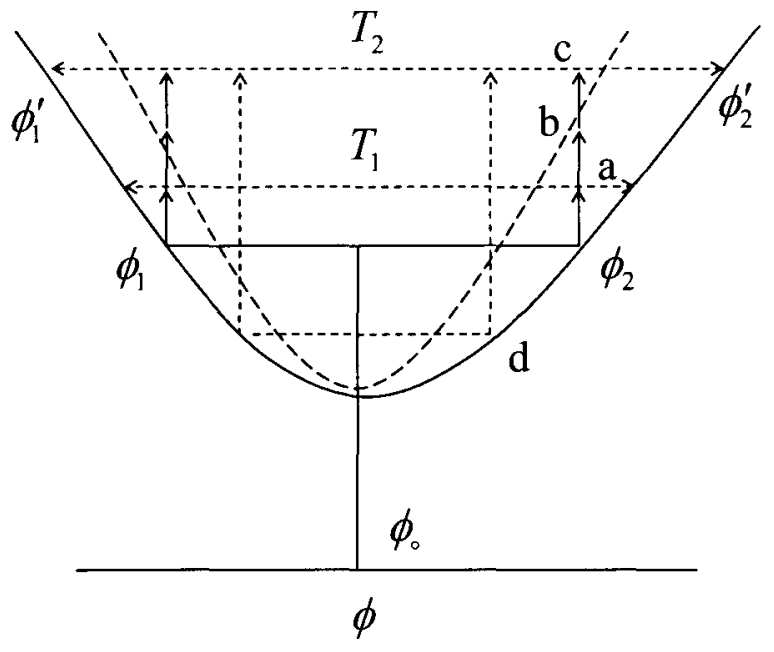

Figure 2.9: Various kinds of double-quench sequences consisting of a first deeper quench and a subsequent deeper quench. In case $a$, both phases become metastable. In case $b$, one phase becomes metastable, while the other unstable. In case $c$, both phases become unstable. In case $d$, phase inversion is induced by the second quench [86]. The resulting morphology is shown in Figure 2.8. 
This strong violation of the local equilibrium after the formation of a sharp interface is never seen for spinodal decomposition under a small fluidity condition. In the late stage of phase separation however, they claimed to have seen spontaneous secondary phase separation of nucleation-growth type at the beginning. For $t>40$, one can clearly see the birth of small white and black droplets in the large black and white domains, respectively which tend to rearrange in a SD-type droplet formation (Figure 2.10). This nucleation-growth probability is dependent upon the noise level (see 3.1) because the nucleation is an event overcoming the energy barrier by thermal noises. Hashimoto et al. [90,91] performed time-resolved light scattering experiments on the two-step phase separation process for a binary polymer mixture of deuterated polybutadiene and protonated polyisoprene with the critical composition (Figure 2.11).

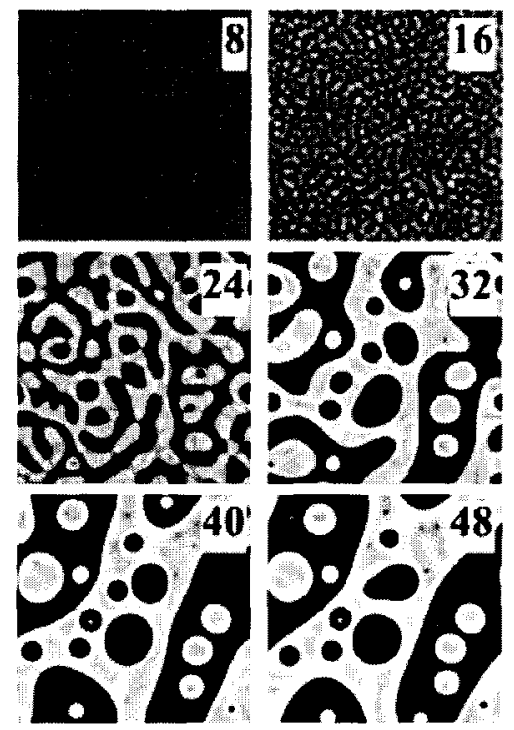

Figure 2.10: Two dimensional pattern evolution during bi-continuous spinodal decomposition for a symmetric composition under a high-fluidity condition at different time steps [87]. 
A very important point which is the core of the research on double phase separation, however, is that this mechanism has been observed only for bi-continuous (critical) phase separation in nearly symmetric mixtures showing hydrodynamic coarsening (which will be explained later) under deep quench conditions and has never observed for off-symmetric mixtures having droplet morphology (off-critical). Tanaka [95] verified this phenomenon experimentally for both symmetric and off-symmetric solutions in a mixture of oligomers of €-caprolactone (OCL) and styrene (OS).

(a) Phase Diagram

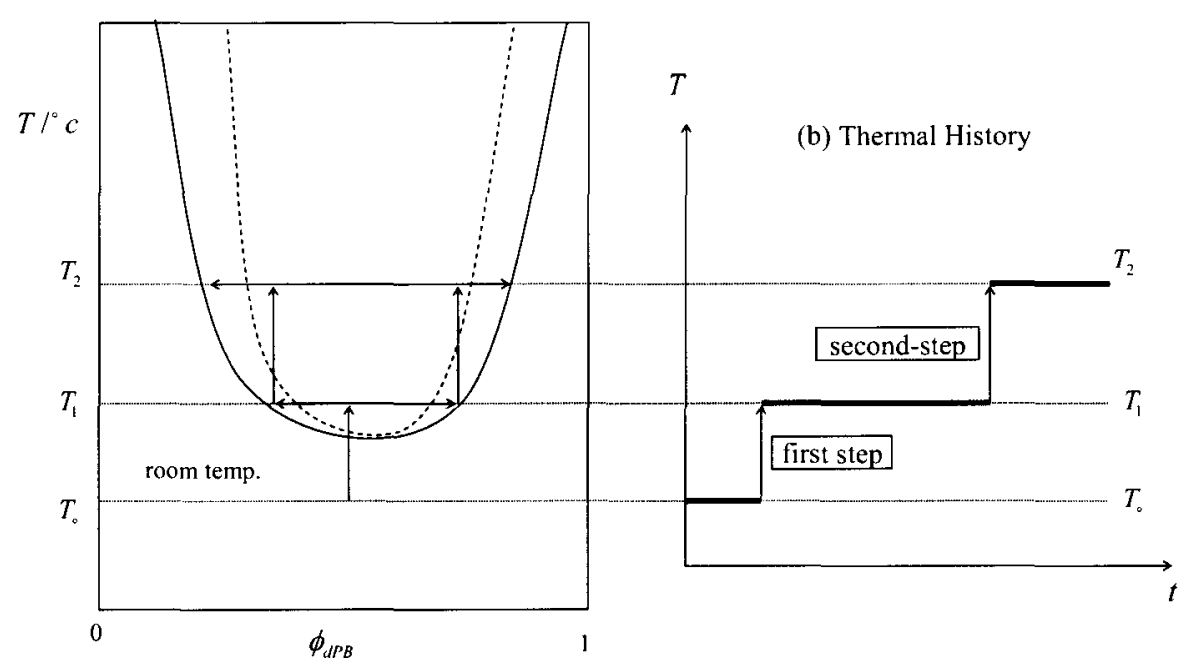

Figure 2.11: (a) Phase diagram of a binary mixture of dPB-hPI. The two-step temperature jump was performed according to the scheme indicated by arrows. (b) Thermal history applied to the two-step phase separation experiments. The first-step SD process at $T_{1}$ (542 $\left.{ }^{\circ} \mathrm{C}\right)$ lasted for $t_{0}$ minutes and subsequently the second-step $T$-jump from $T_{1}$ to $T_{2}\left(570{ }^{\circ} \mathrm{C}\right)$ was performed. The two phases developed after the first-step SD was brought again in spinodal region for the second-step phase separation process [90]. 
He showed that the morphology, as already mentioned, is generally observed for nearly symmetric binary mixtures under deep quench conditions (Figure 2.12). In his experiments, he showed double phase separation morphology (droplet inside the droplet) in confined, symmetric binary mixtures under deep quench conditions. This phenomenon is likely universal for any confined, symmetric binary mixtures. He actually observed the same phenomena in other mixtures such as PVME/water.

Hashimoto et al. [91] also investigated nonlinear time evolution of phase-separating structures in the two-step phase separation process for the same mixture [90] by using a timeresolved light scattering technique.
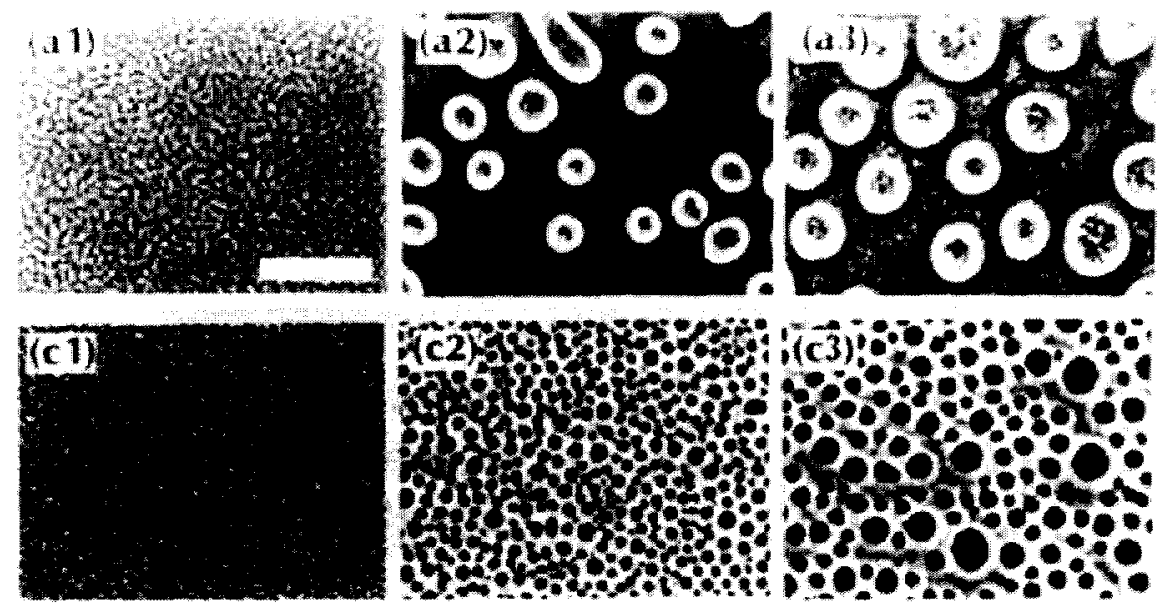

Figure 2.12: Pattern evolution of the OCL/OS mixture. Secondary droplets are more clearly observed for a deeper quench of nearly symmetric mixtures and never observed for offsymmetric mixtures having droplet morphology under any quench conditions. This can be easily confirmed by comparing Figs. 2(al)-2(a3) with Figs. 2(cl)-2(c3) [95]. 
The first-step phase separation via spinodal decomposition was conducted by a temperature jump ( $T$-jump) from $23{ }^{\circ} \mathrm{C}$ to $42{ }^{\circ} \mathrm{C}$, and to the late stage of the SD for varying time periods, $t_{0}$, in order to develop phase-separated domains with varying characteristic size. This phase separation was followed by the second-step $T$-jump to a higher temperature of $70{ }^{\circ} \mathrm{C}$ so that each phase-separated domain was again quenched into thermodynamically unstable region. Nonlinear time evolution processes of phase-separating structures after the second-step SD were explored as a function of size of the initial structures. There, they found the effects of the initial structures on further evolution of phase-separating structure via the second-step SD. Small domains were evolved within the initial domains (defined as large domains) developed during the first-step SD process. They claimed to have succeeded in separating the scattering due to the small domains and that due to the large domains from the observed scattering profile. The separation allowed them to investigate a coupling of the time evolution of the large and small domains and nonlinear pathways for the system to achieve a new equilibrium structure after the second-step SD process.

Henderson and Clarke [53] have studied the early stage of secondary order structure growth from a numerical study of the Cahn-Hilliard-Cook equation, where they use a FloryHuggins-de Gennes free-energy functional to model a polymer blend. Along with numerical simulations, they studied the early stages of double phase separation growth by using the linearized theory of Cahn-Hilliard and quantified the maximum degree of secondary phase separation as a function of quench depth. In these simulations, however, thermal noise was not included; hence thermal fluctuations in the order parameter are not accounted for. A direct comparison of their simulations with the experimental study of Sigehuzi and Tanaka 
[96] is thus difficult. In their numerical work; using a finite difference scheme for a spatially and temporally discretized version of the Cahn-Hilliard equation for symmetric binary polymer mixtures; The blend was quenched from a stable state in the one-phase region to an unstable state in the two-phase region where it undergoes spinodal decomposition (Figure 2.13). The mixture was then allowed to coarsen for two different time periods before a second quench was applied to a point further inside the unstable region.

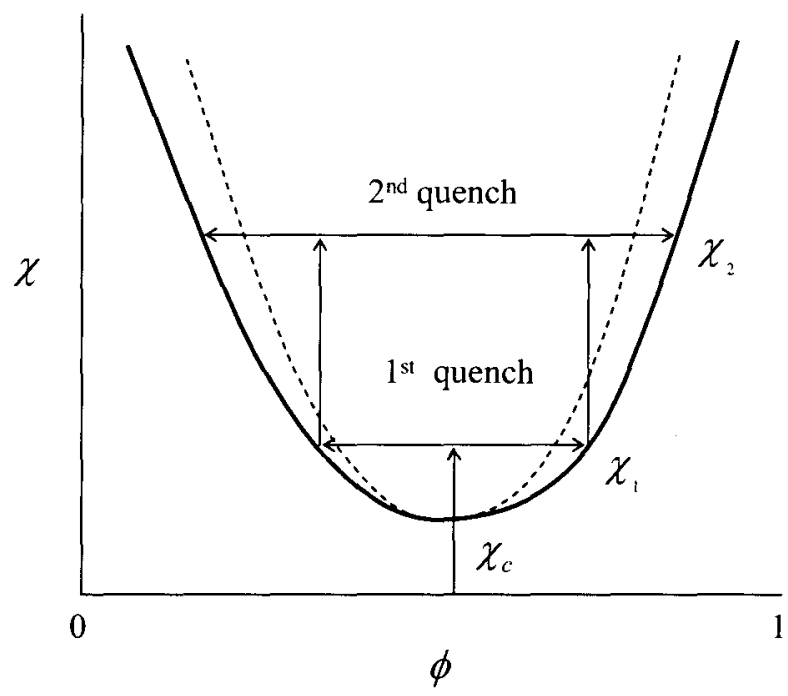

Figure 2.13: Phase diagram illustrating the two-step quench phase separation process in a symmetric binary polymer blend [53].

As expected, the morphology of the first shallow quench resembled the interconnected structure of a critical quench studied by several groups (Figure 2.14). The secondary phase separation has been absorbed back into the larger phases, and the original morphology has been returned (Figure 2.14). The model was based on Cahn-Hilliard-Cook nonlinear 
diffusion equation extended to polymer blends by using Flory-Huggins-de Gennes theory to determine the free energy of mixing per lattice. De Gennes [50] argued that chain connectivity reveals itself as an explicit entropic contribution and proposed that in the presence of fluctuations the Flory-Huggins type free energy functional needs to be supplemented with a square gradient term, the coefficient of which is given by the sum of enthalpic and entropic terms [50]. The work was carried out on a $256 \times 256$ two-dimensional lattice using periodic boundary conditions.

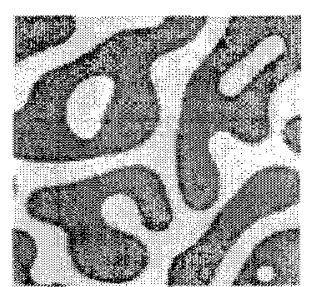

a

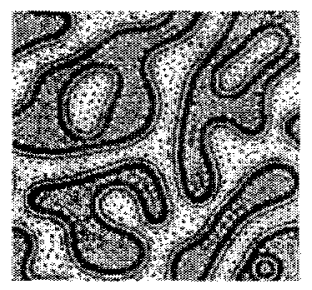

C

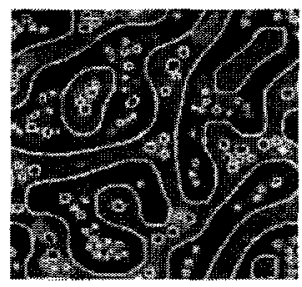

e

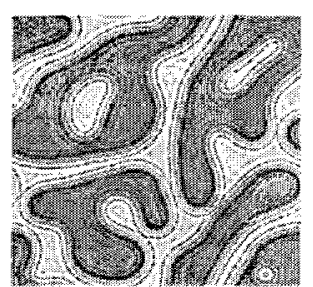

b

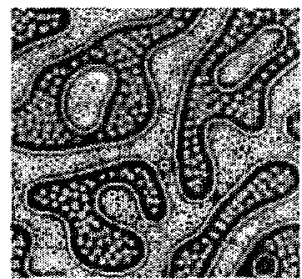

d

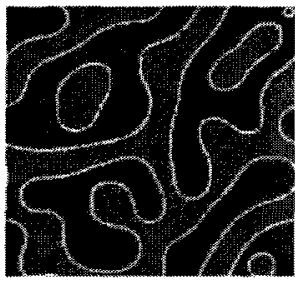

$f$

Figure 2.14: Development of the polymer morphology following the second deep quench into the two-phase region [53]. 
The morphology development was probed by determining the structure factor. They also quantified the maximum degree of secondary phase separation as a function of quench depth. During the initial stages following the second deep quench, smaller secondary domains appeared briefly in the primary domains obtained from the first quench step (Figure 2.14).

Tanaka et al. [96] recently studied phase-separation behavior of an off-symmetric (nearly symmetric) fluid mixture (OS/OCL) induced by a double temperature quench (Figure 2.15). They first quenched a system into the unstable region. After a large phase-separated structure was formed, they again quenched the system more deeply and followed the pattern-evolution process. The second quench made the domains formed by the first quench unstable and led to double phase separation; that is, small droplets were formed inside the large domains created by the first quench (Figure 2.16). The complex coarsening behavior of this type of structure having two characteristic length scales was studied in detail by using the digital image analysis. They found three distinct time regimes in the time evolution of the structure factor of the system. In the first regime, small droplets coarsen with time inside large domains. There; a large domain containing small droplets in it can be regarded as an isolated system. Later, however, the coarsening of small droplets stops when they start to interact via diffusion with the large domain containing them and finally, small droplets will disappear. Thus the observed behavior was explained by the crossover of the nature of a large domain from the isolated to the open system; this is a direct consequence of the existence of the two characteristic length scales. The pattern evolution caused by this type of double quench has been studied experimentally and numerically by several groups $[53,59,60,90,91]$. 


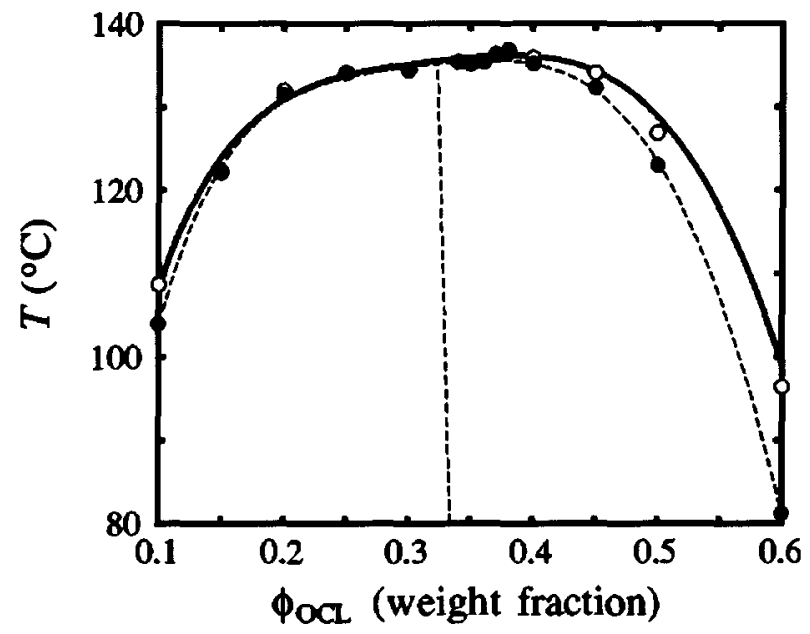

Figure 2.15: The phase diagram of the OS/OCL mixture. The solid and dashed curves are the binodal and spinodal lines, respectively. The dashed line is the composition symmetry line [96].

In previous studies, the structural evolution has been mainly analyzed in the wave-number (q) space. Here they aimed at explaining the characteristic feature of the pattern evolution induced by a double temperature quench by combining both real $(r)$ and wave-number $(q)$ space analyses. On the basis of these analyses, they proposed a simple mechanism for domain coarsening of a phase-separated structure. In their experiment, after the first quench, spinodal decomposition takes place. In the late stage, droplets of the minority phase (the OCL-rich phase) are formed and coarsen with time. They called this structure formed by the first quench; the first-order structure. After the second quench there appeared small OS-rich droplets in the OCL-rich droplets formed by the first quench. They called this structure inside large droplets; the second-order structure. First this subsystem coarsens with time mainly by the Brownian-coagulation mechanism [93-95] accompanying direct droplet collisions and coalescence. 


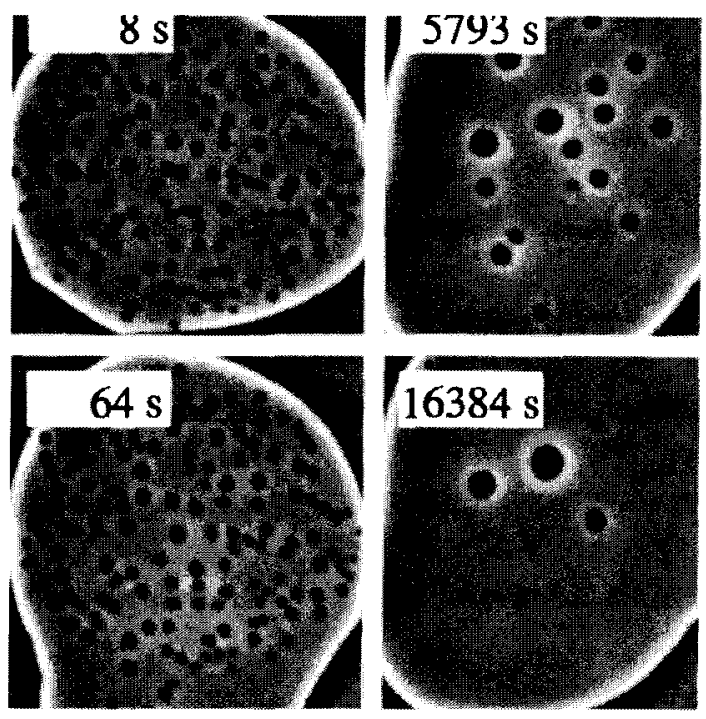

Figure 2.16: Temporal change of the pattern inside a large OCL-rich droplet after the second quench. The size of an image corresponds to $25 \times 25 \mu \mathrm{m}$ [96].

However, the small droplets near the interface of the large OCL-rich droplet start to evaporate and disappear. Eventually all the small OS-rich droplets disappear.

Rullmann and Alig [49] investigated the phase separation process in a critical mixture of polydimethylsiloxane and polyethylmethylsiloxane (PDMS/PEMS, a system with an upper critical solution temperature) by time-resolved light scattering during continuous quenches from the one-phase into the two-phase region. Continuous quenches were realized by cooling ramps with different cooling rates. Phase separation kinetics was then studied by means of the temporal evolution of the scattering vector $(q)$ and the intensity $(I)$ at the scattering peak. They observed a secondary scattering maximum in very late stages of non-isothermal phase separation which was due to secondary demixing. They explained the beginning of secondary demixing by a competition between inter-diffusion and coarsening mechanism. 
In a research performed by Chan et al. [25], they studied through one-dimensional modeling and computer simulation the two-step TIPS in a symmetric polymer blend via spinodal decomposition. The two-step phase separation phenomenon involved the two-step process where the initial quench was allowed to phase separate for a certain period of time before the second quench took place into the unstable region. They showed the second quench occurred at the transition between the early and intermediate stages of spinodal decomposition, which is the time frame when functional polymeric materials with predefined material properties are fabricated. The one-dimensional model consisted of the Cahn-Hilliard theory for spinodal decomposition, and incorporated the Flory-Huggins-deGennes free energy equation, the slow mode mobility theory and reptation model for polymer diffusion. The numerical results simulated frequently reported experimental observations published in the literatures including the observation that secondary phase separation occurs only if the second quench is sufficiently deep. Furthermore, their numerical results indicated that a dimensionless diffusion coefficient may be used as a parameter to control the formation and evolution of the phase-separated regions during spinodal decomposition as a way to tailor-make functional polymeric materials with predefined material properties.

Chakrabarti et al. [73] also numerically studied a two-step quench process in an asymmetric binary mixture. The mixture was first quenched to an unstable state in the two-phase region. After a large phase-separated structure was formed, they again quenched the system deeper. The second quench induced the formation of small secondary droplets inside the large domains created by the first quench. They characterized this secondary droplet growth in terms of the temperature of the first quench as well as the depth of the second one. The 
comparison of Chakrabarti's model with the so far performed modeling and experiments shows that the droplets however; are not conspicuous in their snapshots and since the phase diagram is not provided it is not clear how asymmetric the system chosen to be modeled is. As already mentioned; secondary droplets have been just observed for a deeper quench of symmetric or nearly symmetric mixtures and never observed for off-symmetric mixtures having droplet morphology under any quench conditions. This can be easily confirmed by comparing Figs. 2(al)-2(a3) with Figs. 2(cl)-2(c3) of Figure 2.12 provided experimentally by Tanaka [95] and numerically by others $[25,52,53]$ but since the numerical model they have used includes thermal noise, they believe to have studied the dependence of secondary droplets on the depth of the first and second quenches in a natural way.

\subsubsection{Critical Double Quench Mechanism}

Figure 2.17 schematically shows the two-step TIPS method for a polymer solution with a symmetric phase diagram $\left(N_{1}=N_{2}=1\right)$. Initially, a polymer and a solvent material form a homogeneous solution at some average concentration $\left(c_{\circ}=c_{c}\right)$ and elevated temperature $T_{\circ}$ which is denoted by the dot. The temperature is then lowered to $T_{1}$ at a prescribed cooling rate, thus bringing the solution into the unstable region where phase separation occurs by spinodal decomposition. Upon decreasing the temperature the solution thermodynamic driving force increases and the solution separates into two phases in order to minimize the free energy. The tie line (Figure 3.9) connects the two coexisting phases that are both at equilibrium. At the equilibrium stage, solvent concentration has increased to $c^{\prime \prime}$ and the 
polymer concentration has decreased to $c^{\prime}$ while the average initial concentration is still constant $\left(c_{c}\right)$. Since the system is under critical quench the resulting morphology would be interconnected solvent dispersed inside the polymer matrix as observed and modeled by several groups $[12,16,24]$. Phase separation continues until the polymer solidifies. After imposing the second deep quench on the acquired phase separated solution (Figure 2.17), SD-like phase separation reoccurs in the two original, coexisting phases. Then the phaseseparated domains evolve toward the equilibrium composition values as determined by the coexistence curve and the late stages of SD would approach through the observable domain growth.

The second quench is carried out deeper within the two-phase region to temperature $T_{2}$. In the second quench spontaneous secondary phase separation of spinodal decomposition type occurs for both polymer and solvent independently, namely, the birth of small droplets in the domains of polymer and solvent. In the late stage, on the other hand, the large and small domains start to interact with each other through diffusion, and consequently small droplets gradually evaporate from the neighborhood of the interface of the original droplets. The deeper the depth of the quench, the greater the degree of secondary phase separation, the finer the secondary morphology and the longer it takes to return the morphology to its original $T_{1}$ state. The small droplets newly appeared grow with time in both original droplets (solvent-rich phase) and polymer matrix, and at the same time they disappear first from the interfacial region. The secondary structure is absorbed back into the primary structure while the primary structure coarsens. 


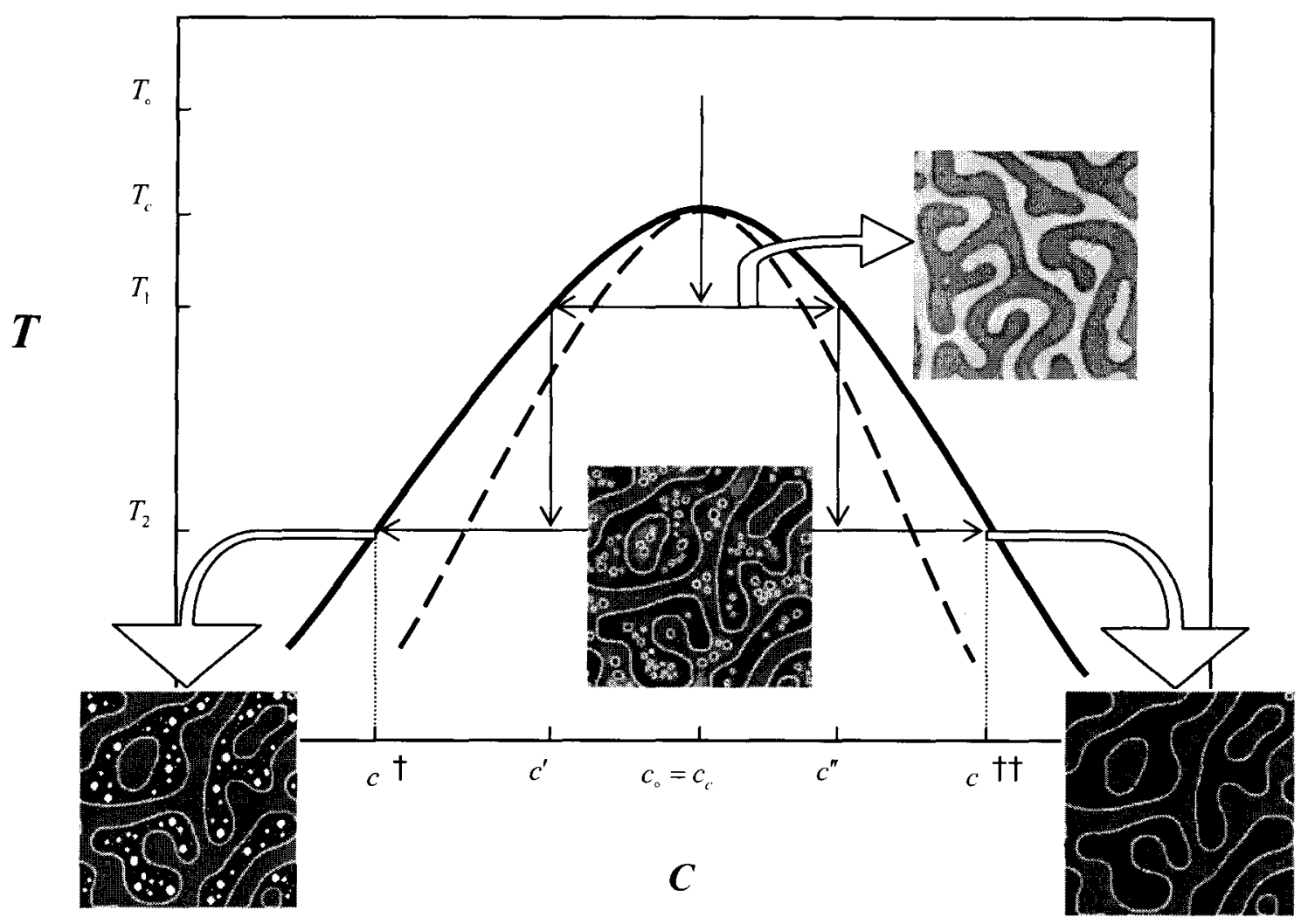

Figure 2.17: Schematic representation of TIPS in a two-step critical quench in a symmetric polymer solution. Both polymer and phase separated solvent obtained from the first quench undergo another phase separation independently.

In phase separation under a critical quench, the total interface area of the system is significantly reduced within a short time by the fast hydrodynamic coarsening driven by interface tension [17]. It is well known that the increase of the domain size is mainly caused by the hydrodynamic flow and diffusion [145]. Since the hydrodynamic interface motion is much faster than the concentration diffusion, the hydrodynamic flow due to interface motion causes only the geometrical coarsening and does not accompany the concentration change. 
That is, the hydrodynamic coarsening can be too quick for concentration diffusion to establish the local equilibrium. This should cause double quench effects, which we call interface quench effects. Eventually coarsening of the domains occurs to reduce the interfacial area. It is also known [139] that there is an enormous acceleration of the domain coarsening for phase separation of a critical polymer mixture. The mechanism of the unusual fast growth of domains on the other hand is not yet understood.

\subsubsection{Off-Critical Double Quench within SD Mechanism}

Traditionally two-step SD process has also been studied in such a way that mixtures undergo an off-critical quench from a single phase state at a temperature $T_{\text {。 }}$ to temperatures $T_{1}$ and then the second quench to $T_{2}$ inside spinodal region in the phase diagram (Figure 2.18). Similar to the critical quench conditions, here an initial structure which is the single phase state characterized by thermal composition fluctuations at $T_{\mathrm{o}}$ will be transformed into an equilibrium droplet type structure at $T_{1}$ which is a macroscopically phase-separated structure. This process has already been well explored. By changing the temperature from $T_{1}$ to $T_{2}$ due to the increase in the driving force of the phase separation, the structure developed before the second-step temperature jump can be regarded as the initial structure for the second-step phase separation process (Figure 2.18). By the deeper quench involved in the second-step phase separation, an additional thermodynamic instability may occur to result in formation of small domains inside the phase-separated domains developed by the first-step phase 


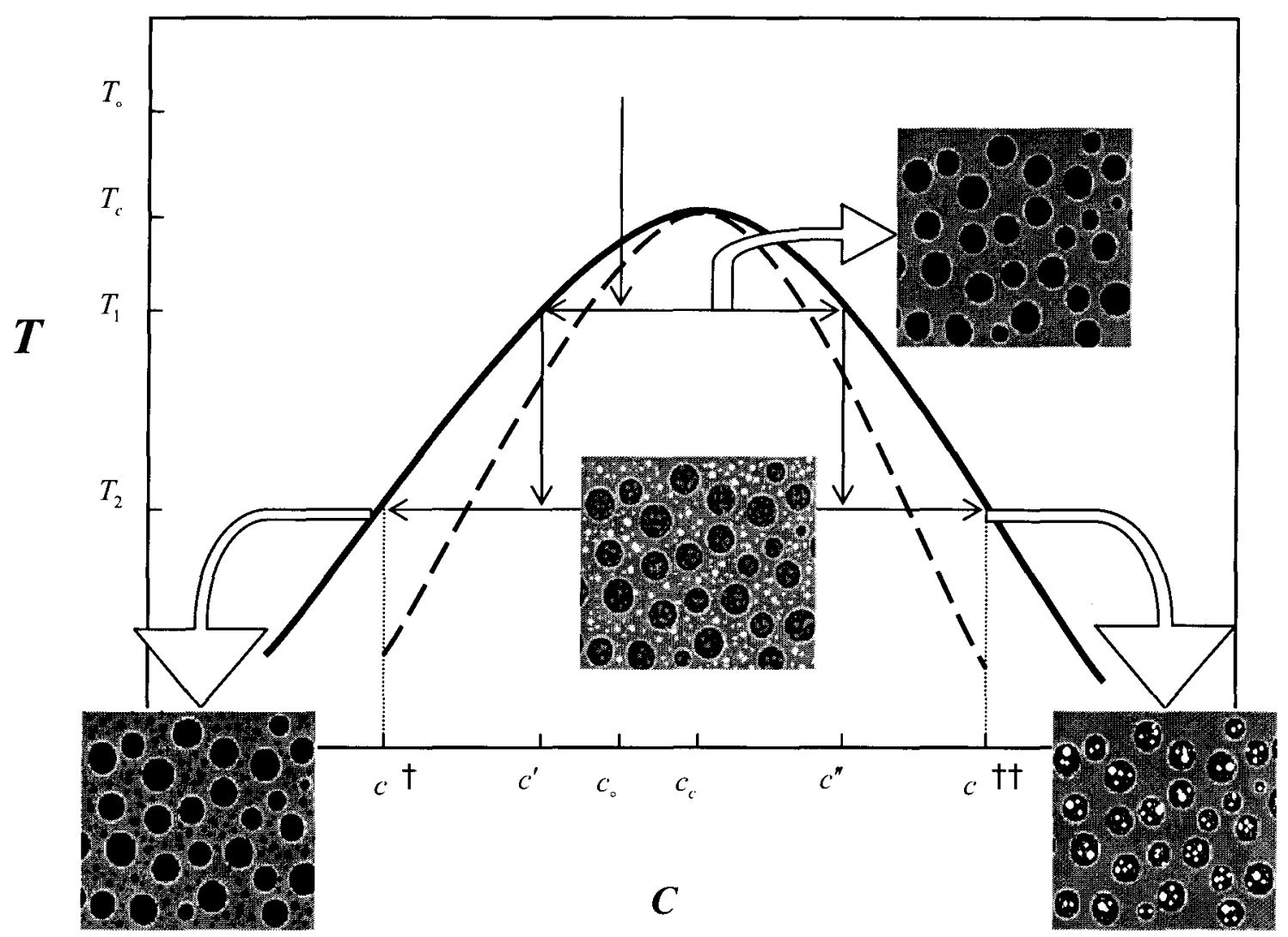

Figure 2.18: Schematic representation of TIPS in a two-step off-critical quench in a symmetric polymer solution. Both polymer and phase separated solvent obtained from the first quench undergo another phase separation independently.

separation. We designate the domain developed before the second-step T-jump as the large domains and the ones developed inside them as the small droplets. It is worthwhile to investigate whether or not the small droplets are developed inside the large domains. The light scattering experiments by Hashimoto et al. [90,91] indicated that the large domains grew further with time after the second step T-jump. It also showed that within the large domains the small droplets grew in amplitude of the composition fluctuations, while keeping 
their size nearly constant, which may be similar to the phenomena observed in the early stage of isothermal single-step SD process. While the large domains further grew with time, they will absorb small droplets in order to decrease excess interfacial energy caused by the presence of the small droplets inside them. Generally, the time-evolution process for the double phase-separation structures via the SD can be classified into the following three stages: (a) early stage, (b) intermediate stage, and (c) late stage. In early stage after the second-step T-jump, the composition profiles are expected to change eventually from $c^{\prime}$ and $c^{\prime \prime}$ to $c \dagger$ and $c \dagger \dagger$ in Figure 2.18 and correspondingly the domain morphology changes by creating small droplets rich in polymer (solvent) in the large domains rich in solvent (polymer), and gets quickly local equilibrium in terms of the composition fluctuations as shown in the figures. The large domain containing small droplets in it can be regarded as an isolated system. Intermediate stage, after the second-step T-jump; corresponds to the stage where the small droplets develop and grow in size and their compositions reach the equilibrium compositions, though these processes themselves are not shown in the figures. Then the small droplets grow via diffusion (droplet collisions) and coalescence. In this stage both small and large domains grow with time. The coarsening of the second-order droplets is much slower than that of usual droplets induced by a single quench phase separation. And finally the coarsening of small droplets stops when they start to interact via diffusion with the large domain containing them.

The grown coarsened small droplets rich in polymer (solvent) inside the large domain of solvent (polymer) are absorbed back into the large domains of polymer (solvent) resulting eventually in the domain structure of the first quench. The structure evolution after this stage 
becomes identical to that for the single-step isothermal SD at $T_{2}$. In stage (c) the small domains grew as a consequence of a diffusion-coalescence process and eventually were absorbed into the large domains, resulting in a reduction of the interfacial free energy. In this process the small domains having larger sizes were found to disappear faster than those having smaller sizes. Figure 2.19 shows the time evolution of the local structure factor of the second-order structure investigated by Tanaka et al. [96]. The peak wave number $q$ decreases with time, while the peak intensity $S(q)$ increases. In stage (b), this tendency stops and both $q$ and $S(q)$ are almost constant with time. Finally, in late stage, which stage, $q$ starts to decrease again while $S(q)$ keeps idling [it finally decays (not shown), reflecting the disappearance of small droplets]. This stage continues until the second-order structure completely disappears.

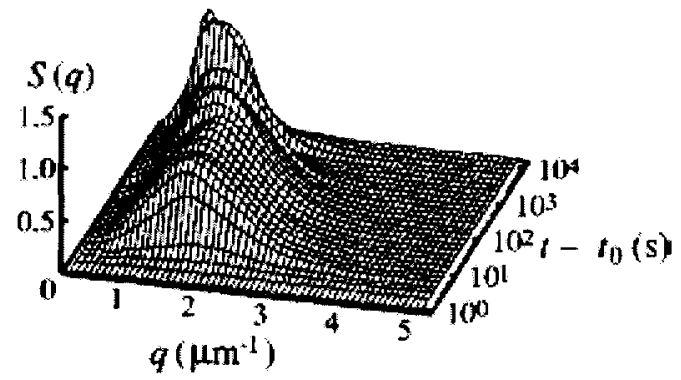

Figure 2.19: Temporal change in the structure factor of the second order structure [96].

In the first two stages, a subsystem inside a large first-order droplet can be regarded as an isolated system, while in the last regime it can no longer be regarded as an isolated one and it 
strongly interacts with the surrounding matrix. It should be noted that double phase separation can be induced even by a single quench [140] when the diffusion cannot catch up with the fast hydrodynamic coarsening. There the original phase-separated structure is completely reorganized. This phenomenon would not happen under a symmetry-preserving quench, namely under a critical quench condition in a symmetric phase diagram. Further studies are highly desirable for clarifying the mechanism dominating phase separation ordering under various multiple quenches and applying the phenomena to the structural control of material.

Figure 2.20 schematically summarizes the whole procedure of a two-step deep quench in a TIPS method. The thick darker arrows around each droplet indicate the diffusion flux induced by the concentration gradient [96].
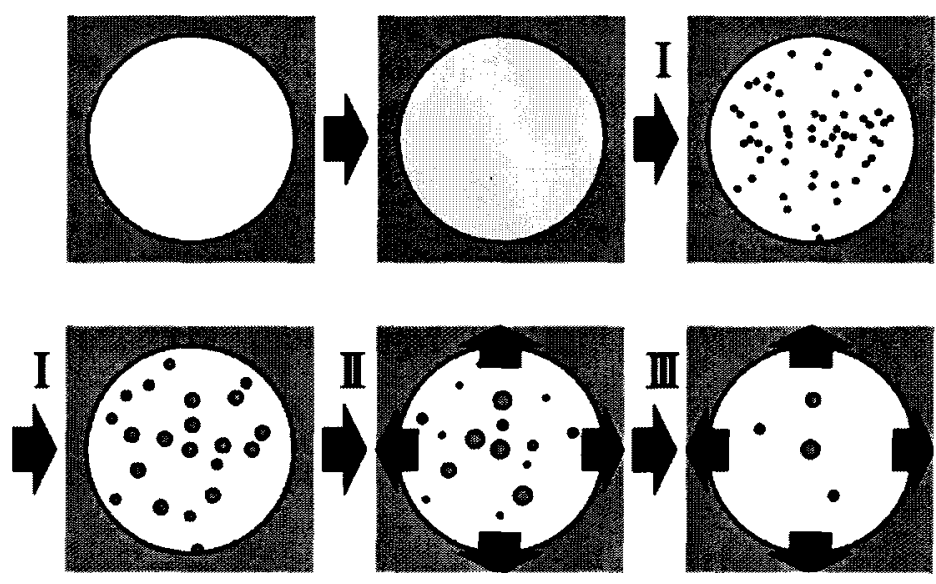

Figure 2.20: Schematic figure describing the pattern evolution after a double quench. The thick black arrow indicates the diffusion flux induced by the concentration gradient [96]. 


\subsubsection{Cavity Structure}

Recent theoretical studies [64,134-139] have addressed a new pattern formation originating from a double quench by carrying out numerical simulations of model systems. Fialkowski and Holyst [83] have studied a two-step quenching process where the first quench is inside the unstable region of the phase diagram but the second quench is above the spinodal line. The decay of the peak of the structure factor is studied in their simulations. In this thesis however, we consider a completely asymmetric binary polymer solution and characterize the secondary droplet growth after the second quench through the nucleation and growth region. Ohshima et al. [64] prepared a unique porous polymeric film by drying a ternary polymer solution: a polystyrene (PS), polyethylene glycol (PEG), and toluene solution. Highly ordered micropores, ranging from 5 to $12 \mu \mathrm{m}$ in diameter, were formed on the film surface, and the rim of each micropore was surrounded by a ring of PEG.

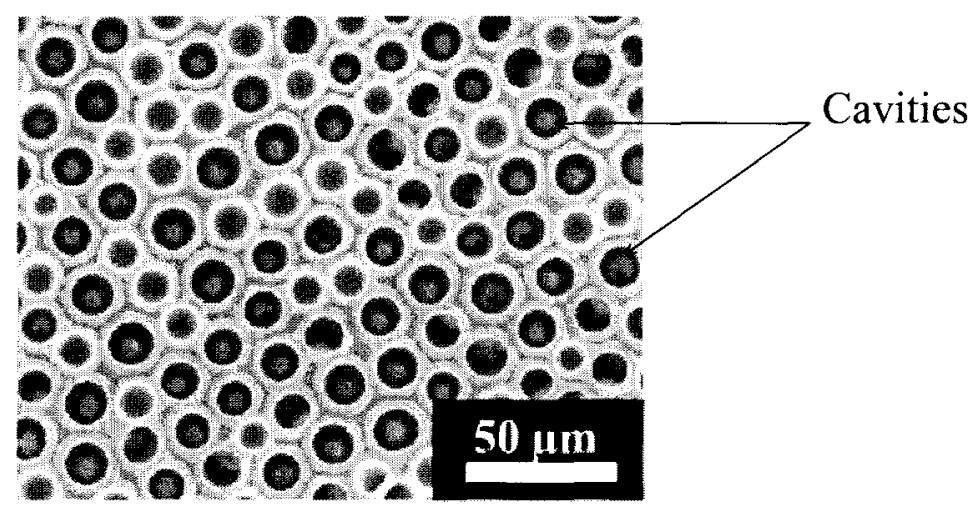

Figure 2.21: SEM micrographs of PS/PEG200 (70/30) (w/w) cast from $90 \mathrm{wt} \%$ toluene solution surface micrograph (notice the cavity structure) [64]. 
The effects of the weight ratio of the polymer blend and molecular weight of the polymer (PEG) on the porous structure were investigated. Based on the visual observation and light scattering measurements, the formation mechanism of the cavity (core-shell) structure was speculated to be a two step phase separation: the phase separation into PEG-rich and PEGpoor (i.e., PS-rich) phases occurred first at the surface area of the ternary solutions, where polymers were condensed due to solvent evaporation. The PEG-rich phase became droplets and had an ordered structure on the surface. The PEG-poor phase became a matrix where PS and solvent coexisted as a single phase solution. Secondary phase separation then followed in the PEG droplets, which was induced by further solvent evaporation, and formed into solvent-rich and PEG-rich domains within the droplets. Solvent evaporation and secondary phase separation created a cavity (core-shell) structure in each PEG droplet structured on the film surface (Figures 2.21 and 2.22).

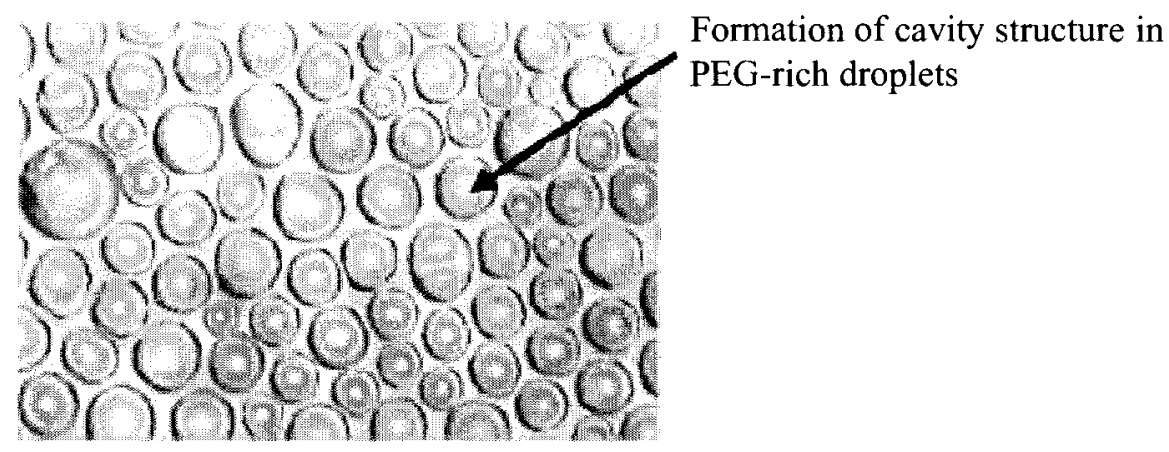

Figure 2.22: Pictures taken with a microscope in the visual observation mode to explain the formation of the core-shell structure [64]. 
They also numerically concerned the two-step SDPS (surface directed phase separation) in asymmetry polymer mixtures by coupling the Flory-Huggins-de Gennes equation with the Cahn-Hilliard-Cook equation. The morphology and evolution dynamic of the phase structure, especially the secondary domain structure, were analyzed in detail. The simulated results as well; demonstrated a core-shell structure in the bulk and a light concentration drop in the wetting layer induced by the second quench with deeper quench depth when the minority component is preferred by the surface.

Yan et al. [78] investigated numerically surface-directed phase separation via a two-step quench process in asymmetry polymer mixtures by coupling the Flory-Huggins-de Gennes equation with the Cahn-Hilliard-Cook equation. Two distinct situations, i.e., the minority component is preferred by the surface and the majority component is preferred by the surface, were discussed, respectively. The morphology and evolution dynamics of the phase structure, especially the secondary domain structure, were analyzed. The simulated results demonstrated that different secondary domain structures in these two situations can be induced by the second quench with deeper quench depth, which can be used to modify phase morphology (Figure 2.23).

Clarke [54] investigated the two step dissolution-quench process in polymer blends. Particles of one type of polymer were allowed to dissolve in a matrix of a dissimilar polymer. Prior to complete dissolution the blend was quenched into the two-phase region, such that phase separation took place. The method is basically similar to the concept of a two step quench 


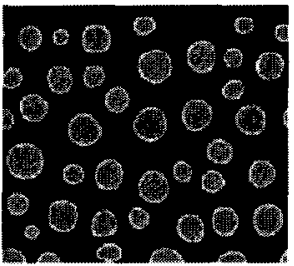

$\tau_{2}=0$

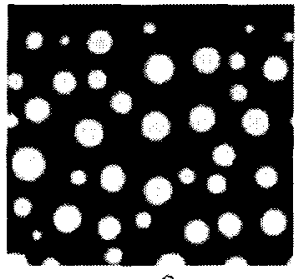

$\tau_{2}=0$

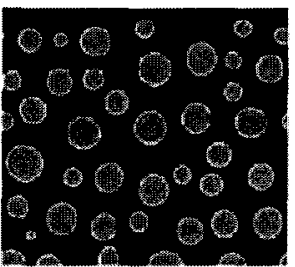

$\tau_{2}=10$

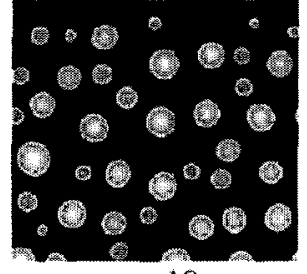

$\tau_{2}=10$

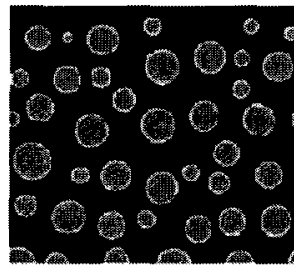

$\tau_{2}=20$

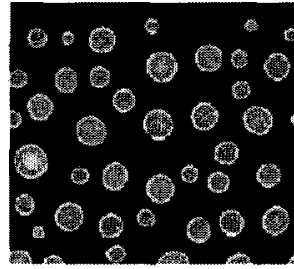

$\tau_{2}=20$

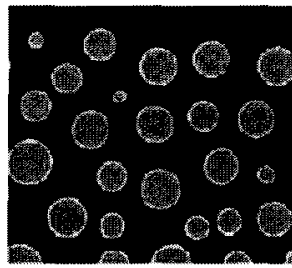

$\tau_{2}=50$

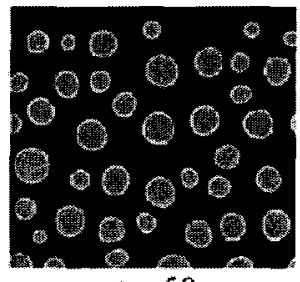

$\tau_{2}=50$

Figure 2.23: Development of the polymer morphology following a second shallow (a) and deep quench (b) (notice the cavities inside the droplets for deep quench at time $\tau_{2}=10$ ) [78]. It also showed that a core-shell structure in the bulk and a light concentration drop can be induced by the second quench with deeper quench depth.

process, studied experimentally by Okada et al. $[59,60]$, Tanaka $[86,87]$ and Hashimoto et al. [90,91]. In his model he started with undissolved particles dispersed in a matrix, with the bulk composition being such that the blend is miscible. During dissolution the interface between the particle and the matrix broadened with time and eventually disappeared. The blend was then quenched into the unstable region, inducing phase separation. He modeled the kinetics of this process by using Cahn-Hilliard theory. The free energy used for the mixture was given Flory-Huggins theory. The model was in two dimensions, using periodic boundary conditions. Although his model is not exactly the same as the others but the resulting morphology is the characteristic of a double quench phase separation (Figure 2.24). 


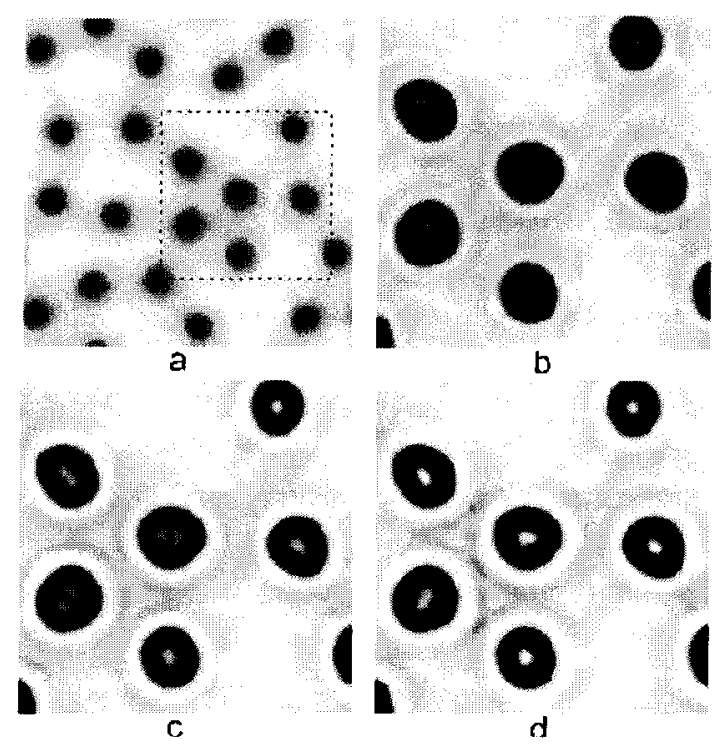

Figure 2.24: Development of the morphology following the quench into the spinodal region. To highlight the existence of cores rich in component $\mathrm{A}$, only a selected $128 \times 128$ area of the simulation, corresponding to the boxed region in (a) is shown in (b) to (d). The grey scale range, which is the same for all snapshots, is such that white represents regions of pure A, and black represents regions of pure B [54].

Some groups experimentally approached the same morphology by different pathways. Hourston et al. [134] experimentally prepared droplets with cavities by a system exhibiting lower critical solution temperature (LCST) phase behavior. On increasing the temperature two times (deep at the second time), phase separation occurred. They divided the core shell structure in the study into three parts: a. the core, b. interface between the core and the shell, c. and the shell. The system was composed of polymethyl methacrylate (PMMA) and PVAc. In their experiment they proposed a quantitative method which can be used to study macromolecular diffusion in the interfacial phase of core-shell droplets. The macromolecular 
diffusion behavior during phase separation was confirmed experimentally. Sun et al. [135] also investigated the same morphology formation mechanism for a mixture of PBA/PVAc. At first, PBA was formed outside the PVAc droplets and then migrated to the inside of PVAc droplets. According to the calculation of the total interfacial free energy change in different possible migration pathway, they proved that the migration pathway of the chains in the experiment was in agreement with the principle that the interfacial free energy gradually decreases. Ishizu [136] independently observed the phase-separated microdomains of cavity microspheres in a PMMA matrix. This micrograph (Figure 2.25) shows clearly the structure of the cavity microspheres with a narrow size distribution. Meijer et al. [138] used two different blending routes, using chemically induced phase separation (or RIPS) to prepare rubber modified PS and PMMA blends.

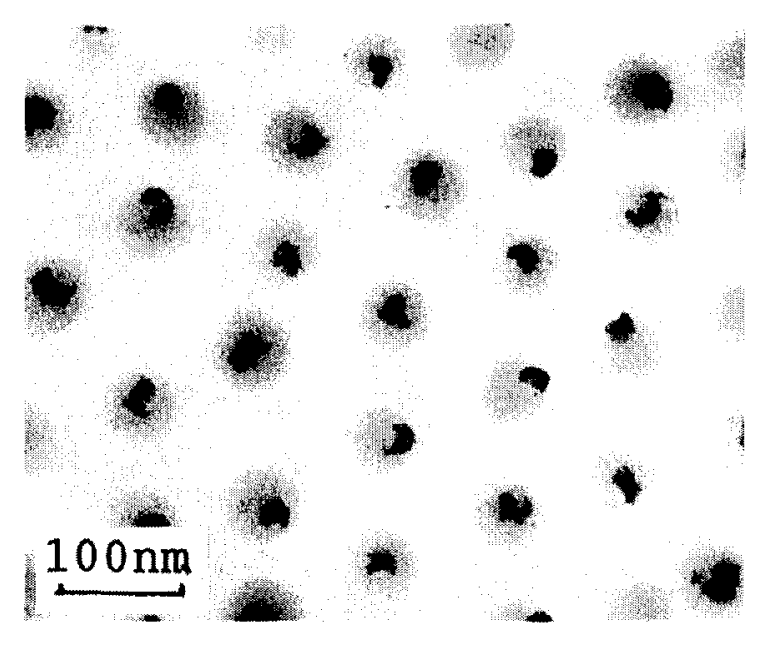

Figure 2.25: TEM (transmission electron microscopy) micrograph of cavity microsphere [136]. The cavities inside the droplets are clearly evident. 
In both cases, the morphology was tailored during the coarsening process after phase separation either by controlling the system viscosity or the reaction rate. The morphology could not be realized via conventional processing techniques like physical blending, and in their study, chemically induced phase separation was used as a route for obtaining a fine dispersion of rubbery particles in polystyrene (PS) and PMMA which resulted in a significant increase in impact toughness. In their work, deformation tests at different rates were conducted along with morphological studies using time-resolved X-ray scattering, to reveal the underlying microscopic deformation processes (Figure 2.26). Huang et al. [139] have studied the phase separation process in a mixture of a liquid crystal material and two different monomers A and B undergoing a PIPS process. Phase equilibrium was theoretically analyzed using a Van der Waals free energy density approach.

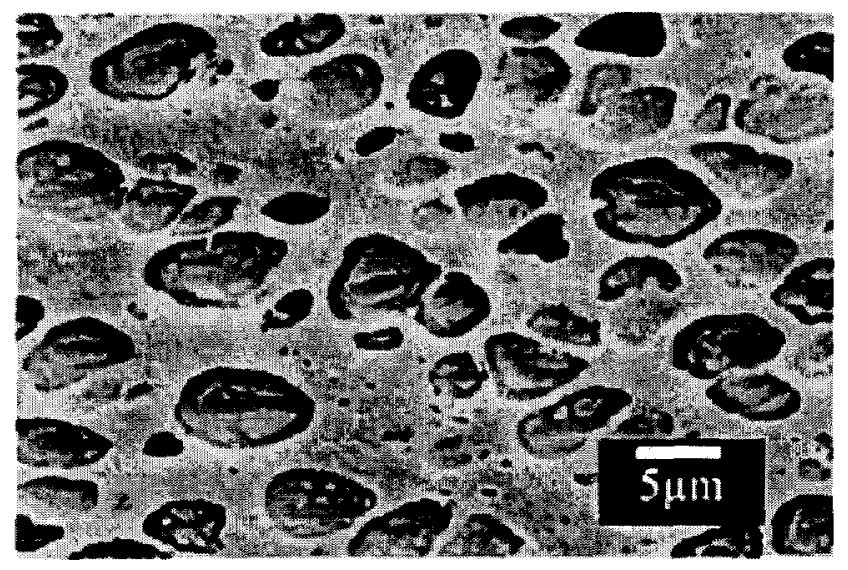

Figure 2.26: Morphology of polymerized PMMA/epoxy 50/50 blends, start temperature of polymerization profile: $60^{\circ} \mathrm{C}$ [138]. 
Their results indicate that the more slowly reacting monomer will be found at higher concentration at the droplet boundary in the fully cured polymer dispersed liquid crystal (cavity). They also used experimental evidence to show that in the homogeneous mixture, component A polymerizes first and this leads to phase separation. Two phases are formed, a more viscous matrix containing mostly B and liquid crystal. At later times B will start to polymerize and then two different processes may occur: a secondary phase separation within the droplets, or the reaction of B at the droplet surface. 


\section{Chapter 3}

\section{Theoretical Background}

This chapter introduces the basic phase separation method and theory of spinodal decomposition and nucleation and growth. We describe a novel mechanism to form binary polymer solutions due to temperature variations. Phase diagram for polymer solutions are presented in detail. The experimental techniques to obtain these thermodynamic phase diagrams are introduced as well. Polymer solutions are differentiated according to Gibbs free energy of mixing $\Delta G_{M}$. The majority of polymer solutions are immiscible. These heterogeneous polymer solutions have a positive $\Delta G_{M}$ value. In some cases, they are soluble. However, they tend towards phase separation to form multiphase at some temperature and molecular weight. In many applications, miscibility of the phases is not desired or required. Therefore, phase separation methods have been one of the practical methods to obtain multi-component polymer solutions. For example, the desired morphology of the solutions can be obtained by controlling the polymer concentration and processing conditions such as temperature, shear rate and pressure. In order to obtain the heterogeneous mixtures and control their phase morphology, it is fundamentally important to understand the phase separation method. In addition, phase separation is an important field of polymer formation, modification and processing. 


\subsection{Phase Separation Thermodynamics}

Thermodynamics is a fundamental factor in determining polymer solutions miscibility $[7,8]$. Based on the second law of thermodynamics, miscibility takes place when the Gibbs free energy of mixing is negative; i.e. $\Delta G_{M}<0$. In addition, the second partial derivative of free energy with respect to composition is positive; i.e. $\left[\partial^{2} \Delta G_{M} / \partial c^{2}\right]>0$ where $c_{i}$ is the volume fraction of the $i$ th component. The Gibbs free energy of mixing is expressed as:

$\Delta G_{M}=\Delta H_{M}-T \Delta S_{M}$

where $\Delta H_{M}$ and $\Delta S_{M}$ are the enthalpy and entropy of mixing, respectively. $T$ is the temperature. Generally, the distinctive property of polymers is their large molecular weight that can be used to control the miscibility of a multi-component mixture. The mixing entropy $\Delta S_{M}$ of a large molecular weight polymer almost equals zero. On the other hand, the enthalpy of mixing $\Delta H_{M}$ is always positive in most polymers, at least for non-polar polymer systems [54]. Therefore, the Gibbs free energy of mixing is seldom negative, which means that phase separation always occurs in polymer solutions. In the case of binary polymer solution systems, the Gibbs free energy of mixing $\Delta G_{M}$ versus volume fraction of a polymer diagram can be constructed as a function of temperature. Figure 3.1 shows a schematic diagram of $\Delta G_{M}$ as a function of polymer concentration at different temperatures in the upper part. A temperature-polymer concentration phase diagram is shown in the lower part of the figure. 


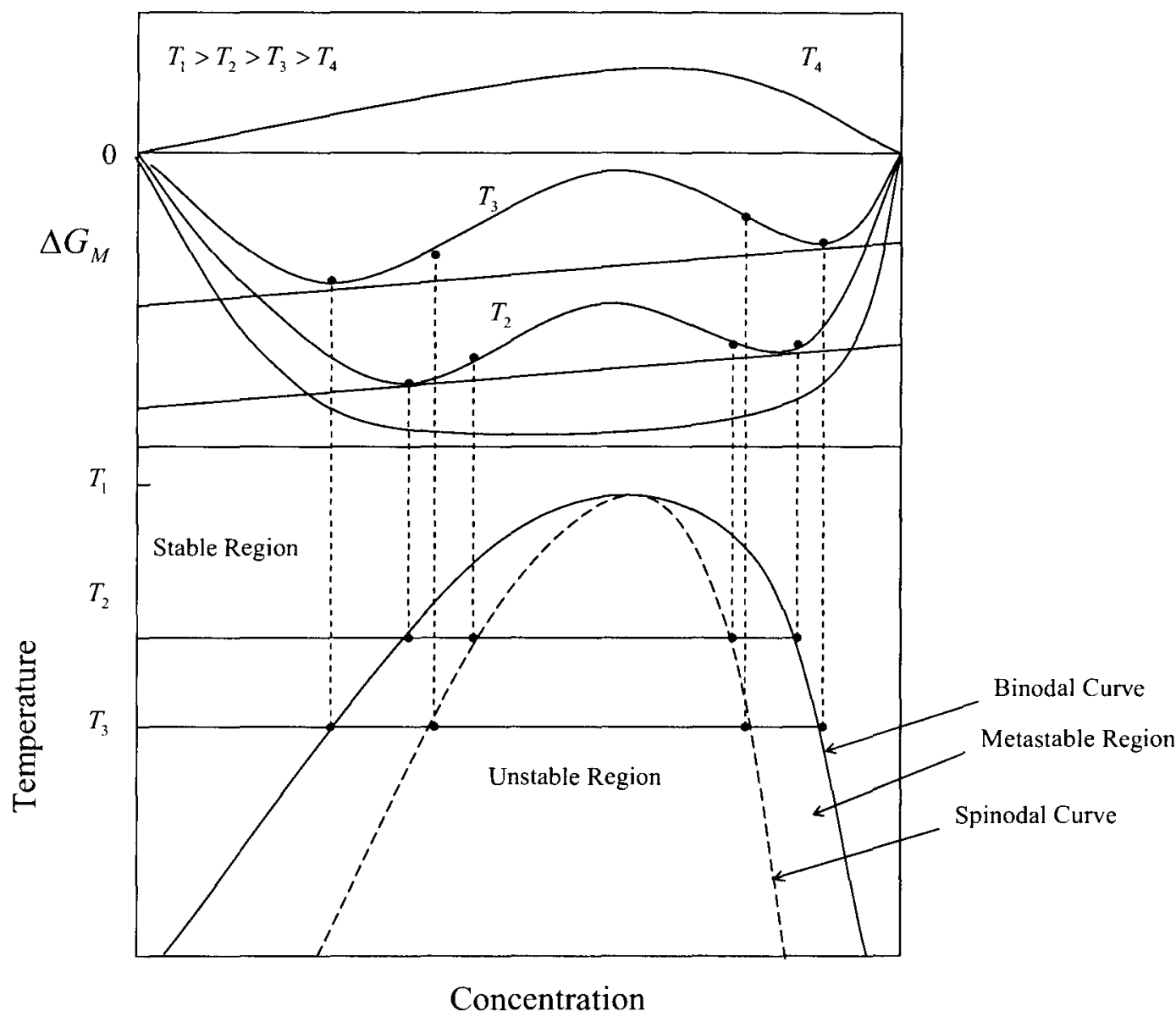

Figure 3.1: Schematic diagram of Gibbs free energy of mixing as a function of polymer concentration [74].

As shown in Figure 3.1, the shape of the free energy of mixing curve and the beginning of phase separation varies by changing the temperature values from $T_{1}$ to $T_{4}$ $\left(T_{1}>T_{2}>T_{3}>T_{4}\right)$. Critical point is also shown in the figure. In phase separation mechanism, the miscible polymer solution at an initial temperature $T_{1}$ goes down into the lower temperatures, eventually becomes a totally immiscible system. 
In the upper part of this diagram, the binodal (cloud-point) curve is formed by determining the projected points, which are shown in Figure 3.1. The straight lines are the common tangential lines for the free energy curve. Meanwhile, the spinodal curve is obtained by the projection of the inflection points. For a mixture of two components involving a polymer and a solvent (PDLC for instance) as well, the Gibbs free energy of mixing $\Delta G_{M}$ must be negative to form a homogenous mixture.

In Figure 3.1 at $T_{1}, \Delta G_{M}$ shows only one minimum over the polymer concentration range. Therefore, the system is completely miscible over the whole range of polymer concentration as shown in the phase diagram. At $T_{2}$ and $T_{3}$, even though $\Delta G_{M}$ is lower than zero for the whole range of polymer concentration, the system is only partially miscible. Since $\Delta G_{M}$ shows two local minima, in order to have the free energy $\Delta G_{M}$ of the system at the overall minimum, the system will separate into two phases with the concentrations of the two phases determined by the tangent points on the $\Delta G_{M}$ curve where

$\left[\frac{\partial \Delta G_{M}}{\partial c}\right]_{1}^{\text {First phase }}=\left[\frac{\partial \Delta G_{M}}{\partial c}\right]_{2}^{\text {Second phase }}$

These two points are called binodal points, and the curve connecting all these points at different temperatures is the binodal curve. The inflection points of $\Delta G_{M}-c$ curve corresponding to $\left[\partial^{2} \Delta G_{M} / \partial c^{2}\right]=0$ are the spinodal points, and the curve connecting these points is called the spinodal curve. The spinodal and binodal curves meet at the critical point. 
The critical temperature, which is the intersection point of the binodal and spinodal curves, is an important quantity for binary polymer solution phase diagrams since

$\left[\partial \Delta G_{M} / \partial c\right]=\left[\partial^{2} \Delta G_{M} / \partial c^{2}\right]=\left[\partial^{3} \Delta G_{M} / \partial c^{3}\right]=0$

In the phase diagram, the region above the binodal curve is the stable region where a homogenous solution can be formed. The region inside the spinodal curve is the unstable region corresponding to $\left[\partial^{2} \Delta G_{M} / \partial c^{2}\right]<0$ where the system spontaneously phase-separates into two co-continuous phases. Between the binodal and spinodal curves, the system may be one phase but not stable, which is called the metastable region, where $\left[\partial^{2} \Delta G_{M} / \partial c^{2}\right]>0$.

At $T_{4}$, the free energy of mixing is larger than zero, and therefore the system is completely immiscible over the whole range of the polymer concentrations. This is not shown in the phase diagram. The phase behavior shown in the lower part of Figure 3.1 is the typical behavior of systems showing an upper critical solution temperature (UCST), where the system enters the two phase region upon decreasing temperature from $\mathrm{A}$ to $\mathrm{C}$. If increasing the temperature brings the system from miscible to immiscible region, it is referred to a lower critical solution temperature (LCST) type of phase behavior. Figure 3.2 shows different combinations of these two types of behaviors. In addition to only UCST (B) and LCST (C), a system can display both types of phase behaviors. This is illustrated by diagram D and E, which shows an island of immiscibility. The UCST and LCST branches in diagram D can merge to form an hourglass shape phase diagram. 
This type of transition has been observed experimentally in polystyrene solutions in acetone [103] where two branches at higher pressures move to each other and merge at lower pressures.
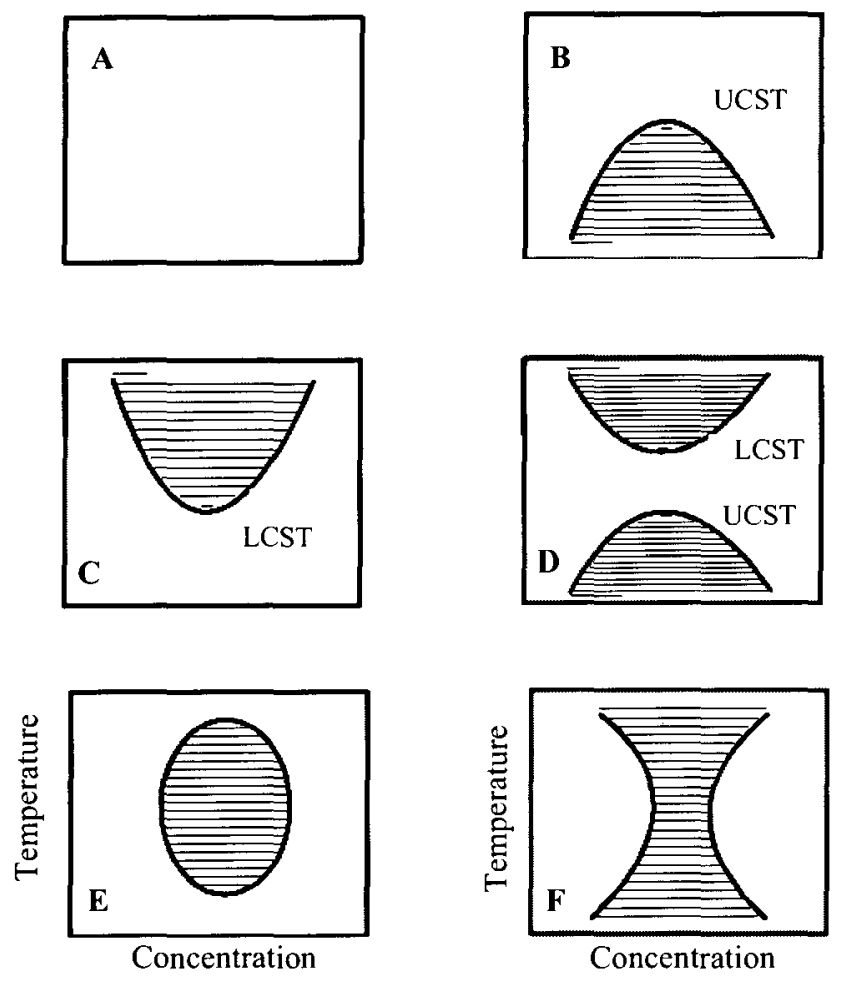

Figure 3.2: Schematic of phase behavior in polymer solutions. Shaded areas represent the two-phase regions and the un-shaded areas represent one-phase regions [105].

\subsubsection{Flory-Huggins Theory}

In this section, we review the derivation of the thermodynamics of solutions of polymers. This theory was formulated independently by P. J. Flory [112] and M. L. Huggin [113] in the 
middle of the last century. The entropy and enthalpy of mixing of two polymers was derived and phase diagrams of polymer pairs were explained on the basis of enthalpy interactions. We will also review contributions of several researchers that have extended this theory to explain the phase diagram that occurs in the literature.

\subsubsection{Entropy of Mixing of Polymer Solutions}

The phase stability of a binary polymer mixture system can be well understood in terms of the lattice theory of Flory- Huggins. Thus, the Flory-Huggins free energy equations (3.6) and (3.8) are the most widely and successfully used theories in phase equilibrium studies [80]. A polymer chain molecule in itself is a large and complicated structure of repeat units and can assume a high number of configurations by itself. Entropy is defined as the degree of randomness of a system. Thus, a polymer chain molecule has higher entropy than an ordinary small molecule. Consequently, a polymer solution is not affected significantly by mixing as the increase in entropy due to mixing is minor. Considering Baltzmann's law of enropy which is:

$\Delta S_{M}=k_{B} \ln \Omega$

where $k_{B}$ is the Boltzmann's constant and $\Omega$ is the number of possible random arrangements in the lattice space; the determination of the entropy of mixing for binary polymer solutions is important for the strictness of the theory and is defined analogous to 
simple liquids. After some mathematical changes, the entropy change of mixing for a polymer solution can be expressed as:

$\Delta S_{M}=-k_{B}\left[N_{1} \ln c_{1}+N_{2} \ln c_{2}\right]$

where $c_{1}$ is now the volume fraction of component one (solute), and $N_{1}$ and $N_{2}$ are the degree of polymerization of the solvent and solute (polymer) respectively. For a low molecular weight solvent $N_{1}=1$. By taking $c_{1}=c$ then $c_{2}=1-c$. Using the constant density approximation yields a correlation between mole fraction and volume fraction in a straight forward manner and by substituting the resultant equation in equation (3.5) yields an expression for the entropy of mixing for a polymer solution:

$\Delta S_{M}=-k_{B}\left[\frac{c}{N_{1}} \ln c+\frac{(1-c)}{N_{2}} \ln (1-c)\right]$

\subsubsection{Enthalpy of Mixing of Polymer Solutions}

According to Flory's approach [113], the enthalpy of mixing $\left(\Delta H_{M}\right)$ for a polymer solution consisting of two components can be calculated by taking the difference between the enthalpy of the solution $\left(H_{1,2}\right)$ and the enthalpy of the pure components $\left(H_{1,1}\right.$ and $\left.H_{2,2}\right)$ to yield the relation:

$\Delta H_{M}=H_{1,2}-\left(H_{1,1}+H_{2,2}\right)$ 
$H_{1,1}, H_{2,2}$, and $H_{1,2}$ are defined using an interaction energy that exists between every two segments. Hence, the total enthalpy of mixing for a polymer solution would be derived as:

$\Delta H_{M}=k_{B} T \chi c(1-c)$

where $\chi$ is temperature dependent Flory's interaction parameter which is a measure of the effective interaction between solvent and solute. For the case of a polymer solution, a variety of effects would have to be taken into account, such as incomplete filling of the lattice sites, chain connectivity, branching and more. It is for this reason that the Flory-Huggins interaction parameter is not generally calculated by this expression. An empirical relationship is used to define the parameter that has a reciprocal dependence on absolute temperature as described by the theory and whose constants can be derived and fitted to the experimental phase diagrams to account for the deviations in both the entropy and enthalpy of mixing in real polymer mixtures [104]:

$$
\chi=A+\frac{B}{T}
$$

where $\mathrm{A}$ and $\mathrm{B}$ constants are determined experimentally and represent the entropic and enthalpic contribution respectively. The entropic contribution accounts for the segmentsegment interactions between the polymers within the mixture. The enthalpic term accounts for the change in energy upon mixing of the polymers as a result of the interactions between segments. The Flory-Huggins interaction parameter is directly related to the critical temperature $T_{c}$ and the interaction parameter at the critical point $\chi_{c}$. 
The critical temperature is then obtained from the following theta temperature relationship with $\chi$ :

$$
\chi=\frac{1}{2}-\psi\left(1-\frac{\theta}{T}\right)
$$

where $\psi$ is a dimensionless entropy of dilution parameter, and $\theta$ is the theta temperature. The theta temperature is where the polymer solution appears to behave as if it is ideal [45]. The $\chi$ parameter measures the solubility of polymer solutions (The values are presented in Appendix B).

\subsubsection{Determination of Phase Diagram}

The Flory-Huggins treatment represents the free energy density of mixing, $f(c)$, as a sum of the configurational entropy and enthalpy of mixing [104]. The starting point of the model is the Gibbs free energy of mixing, $\Delta G_{M}$, given by equation (3.1). The incompressibility assumption, $c_{1}+c_{2}=1$, leads to the reduction of the free energy $\Delta G_{M}$ in a single independent thermodynamic variable $c_{1}=c$ and $c_{2}=1-c$. Gibbs derived a necessary condition for the stability of a fluid phase that the chemical potential of a component must increase with increasing density of that component. In the situation of a two component system this yields the relation, $\left[\partial^{2} \Delta G_{M} / \partial c^{2}\right]>0$. If this condition is not satisfied, then the mixture becomes unstable with respect to any infinitely small composition fluctuations. Also, the total free energy of mixing should be negative for the process to be thermodynamically 
favorable, i.e. $\Delta G_{M}<0$. It is a fundamental principle of thermodynamics that materials always progress towards a state of minimum free energy until equilibrium is reached. This concept is expressed mathematically utilizing the Gibbs free energy of isotropic mixing. According to the F-H theory, entropy of mixing is given by equation (3.6) and enthalpy of mixing can be expressed as equation (3.8). Equation (3.10) introduces the temperature dependence into the F-H equation, thus providing a direct temperature-concentration relationship.

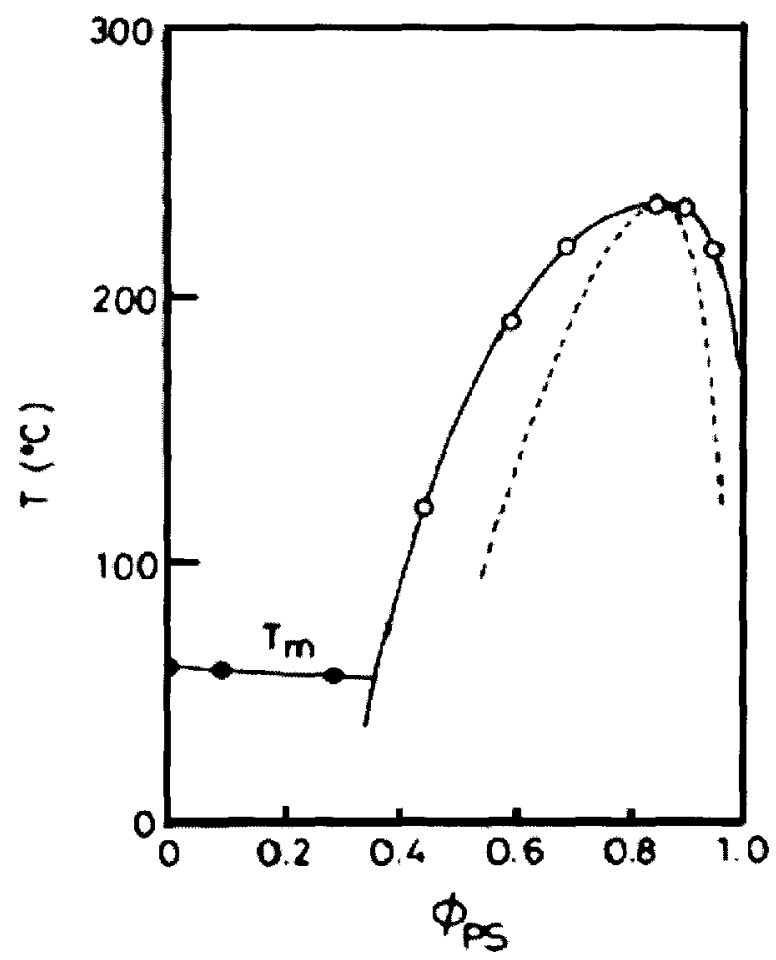

Figure 3.3: Experimentally determined phase diagram of the PCL/PS blend by Tanaka and Nishi [53]. The black circles are the experimental data for the melting temperature and the white circles are the phase separation temperatures obtained from cloud point measurements. Solid and dashed curves are computed using the Flory-Huggins mixing theory [105]. 
The F-H free energy density of mixing is obtained by combining equation (3.1) and (3.6) with equations (3.8) and (3.10), which is expressed as [104]:

$f(c)=\frac{k_{B} T}{v}\left[\frac{c}{N_{1}} \ln c+\frac{(1-c)}{N_{2}} \ln (1-c)+\chi c(1-c)\right]$

where $v$ is the volume of a cell or segment. Based on the condition for phase equilibrium that the chemical potential of each component is the same in all phases at a specified temperature and pressure, the two binodal points are calculated by solving a pair of nonlinear algebraic equations. The chemical potentials of each components, $\mu_{1}$ and $\mu_{2}$ are obtained by taking the first order partial derivative of $\Delta G_{M}$ with respect to $N_{1}$ and $N_{2}$ :

$$
\begin{aligned}
& \Delta \mu_{1}=k_{B} T\left[\ln c+\left(1-\frac{1}{N_{2}}\right)(1-c)+\chi c(1-c)^{2}\right] \\
& \Delta \mu_{2}=k_{B} T\left[\ln (1-c)+\left(1-N_{2}\right) \chi N_{2} c^{2}\right]
\end{aligned}
$$

The conditions for equilibrium between two phases in polymer solutions are expressed by specifying equality of the chemical potentials in the two phases:

$$
\begin{aligned}
& \mu_{P}\left(c^{\alpha}\right)=\mu_{P}\left(c^{\beta}\right) \\
& \mu_{S}\left(c^{\alpha}\right)=\mu_{S}\left(c^{\beta}\right)
\end{aligned}
$$

where the subscripts $\alpha$ and $\beta$ designate two equilibrium phases. The binodal points at a given temperature can be determined by solving equations (3.14) and (3.15) simultaneously.Under the same equilibrium condition, the two spinodal points at the same temperature can also be 
obtained by solving the second order partial derivative of the free energy of mixing with respect to concentration (Figure 3.3) [105,106]:

$\left[\frac{\partial^{2} \Delta G_{M}}{\partial c^{2}}\right]=\frac{1}{c}-\frac{1}{N_{2}(1-c)}-2 \chi$

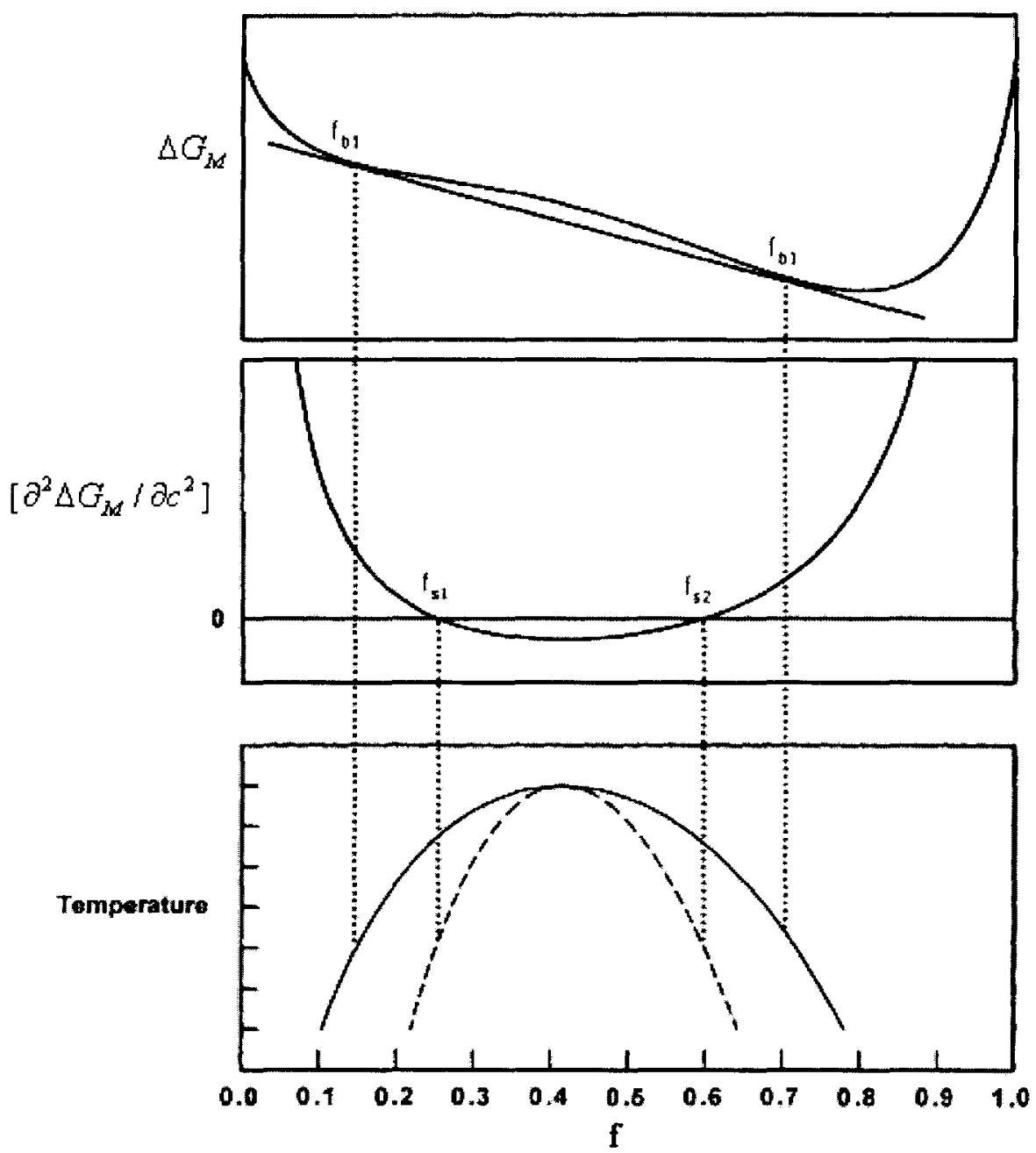

Figure 3.4: Schematic phase diagram with corresponding the free energy of isotropic mixing and its derivative at a certain temperature [107]. 
Figure 3.4 schematically illustrates a free energy curve, its derivative and a hypothetical phase diagram demonstrating the thermodynamic conditions for polymer mixtures. The regime enclosed by the spinodal curve is called the unstable phase, which originates from spinodal decomposition (SD). Metastable phase due to nucleation and growth (NG) belongs to the regime between the spinodal curve and the binodal curve. The critical point can be obtained from the condition for the criticality:

$\left[\frac{\partial^{2} \Delta G_{M}}{\partial c^{2}}\right]=\left[\frac{\partial^{3} \Delta G_{M}}{\partial c^{3}}\right]=0$

By solving equation (3.17), the critical concentration $c_{c}$ and the critical interaction parameter $\chi_{c}$ can be determined.

\subsection{Spinodal Decomposition Theory}

It is of great importance to understand the dynamic aspects of the phase separation as well as the thermodynamic features. Hence, in this section, the basic theories on the phase separation kinetics will be briefly reviewed. Starting from the stable region, the polymer solution can be quenched to the metastable region or unstable region. Depending on the location where the system is brought to, the system will undergo phase separation via two different mechanisms: (a) nucleation and growth, or (b and c) spinodal decomposition (Figure 3.5). Furthermore, SD-type phase separation is grouped into bi-continuous (interconnected) and droplet SD which was already discussed in literature. 
When a polymer solution is quenched critically (passing through critical point of its phase diagram) into the unstable region (Figure 3.5-c) the mechanism of phase separation is proceeded by spinodal decomposition and the resulting morphology would be interconnected or bicontinuous structure (Figures 2.3, 2.4 and 3.6). Figures 1.2 and 3.10 (1.4 and 3.8) schematically illustrates the growth of the concentration fluctuation of one component during phase separation according to SD (NG). In the early stages of SD, periodic concentration fluctuations with wavelength $\lambda$ are built up throughout the sample space and amplitude of concentration fluctuation increases with time, while $\lambda$ remains essentially constant. The wavelength is influenced by the thermodynamic conditions of the solution characterized by the quench depth while the amplitude of the fluctuation is determined by the kinetics and the time of phase separation.

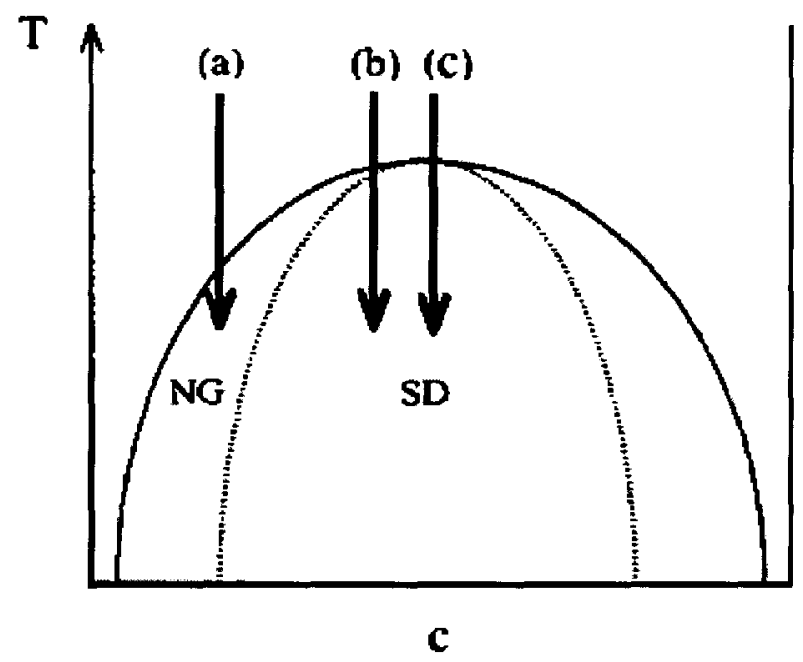

Figure 3.5: Typical phase diagram of a symmetric polymer mixture for the TIPS process. The arrow (a) indicates a temperature change from the homogeneous region into the metastable region (NG) and arrows (b) and (c) represent temperature jumps into the unstable region (SD) off-critically and critically respectively. The solid (dotted) curve represents binodal (spinodal) line. 


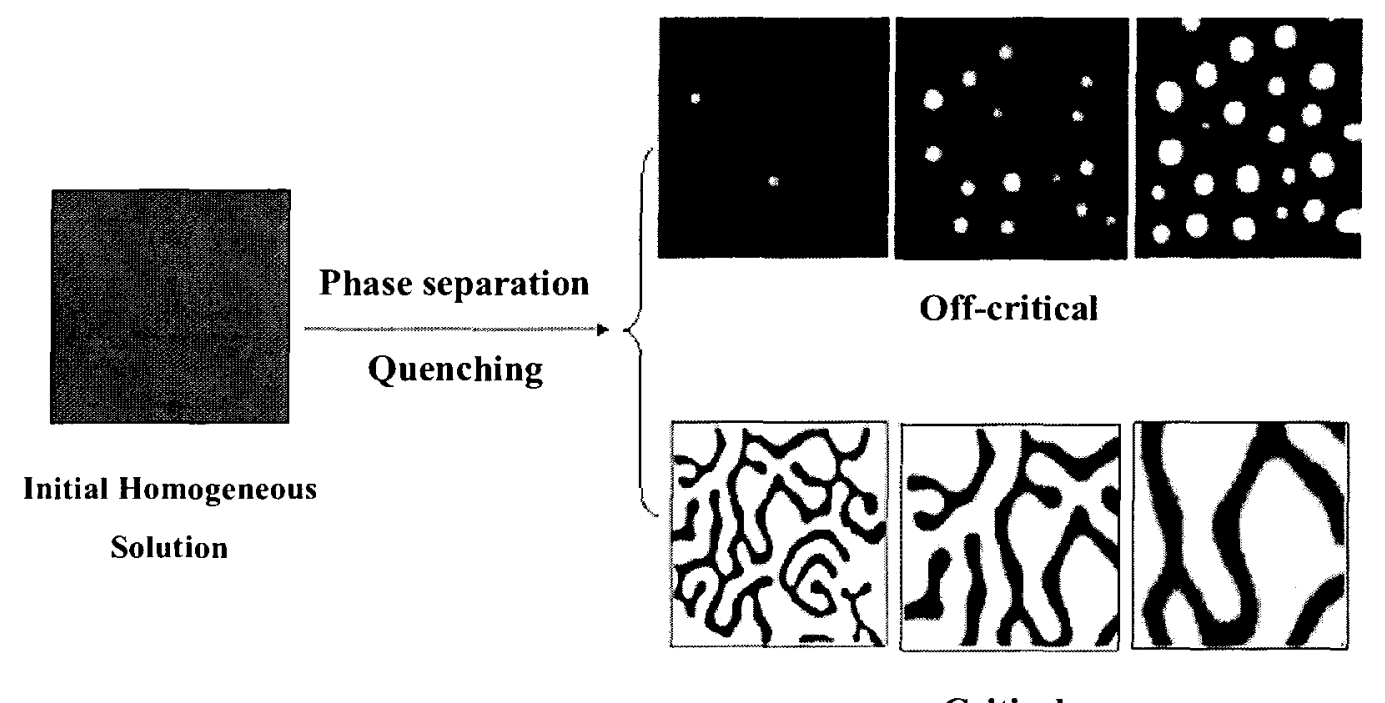

Critical

Figure 3.6: SD-type phase separation droplet (interconnected) morphology resulting from off-critical (critical) temperature quench [34].

Spinodal decomposition refers to the phase separation, which takes place under the condition that the energy barrier is negligible and the compositional fluctuation is even small. It is a kinetic process of generating a spontaneous and continuous phase within the unstable region in Figure 3.5. The time evolution of domain structure in polymer solutions via SD may be classified into three major regimes: (i) early stage, (ii) intermediate stage, and (iii) late stage as illustrated in Figures 1.2 and 3.7. In the early stage of SD, the growth of the fluctuations is weakly nonlinear so that it can be well approximated by the predictions based on the linearized Cahn's theory [36], which may describe the structure in terms of a superpositioning of sinusoidal composition modulations of a fixed wavelength, but random in amplitude, orientation and phase. In this stage of SD, the concentration fluctuations are small at $t_{0}$ and as time increases to $t$, the amplitude of the concentration increases but the wavelength remains constant. 
This region is usually the most difficult to visualize since it happens at such a fast rate. For this reason, the $\mathrm{C}-\mathrm{H}$ equation can be approximated by a linear function [33]. This has proven to be a very useful estimation for the initial stage of SD. In the early stage, Figure 3.7(a), droplet formation occurs.

(a)
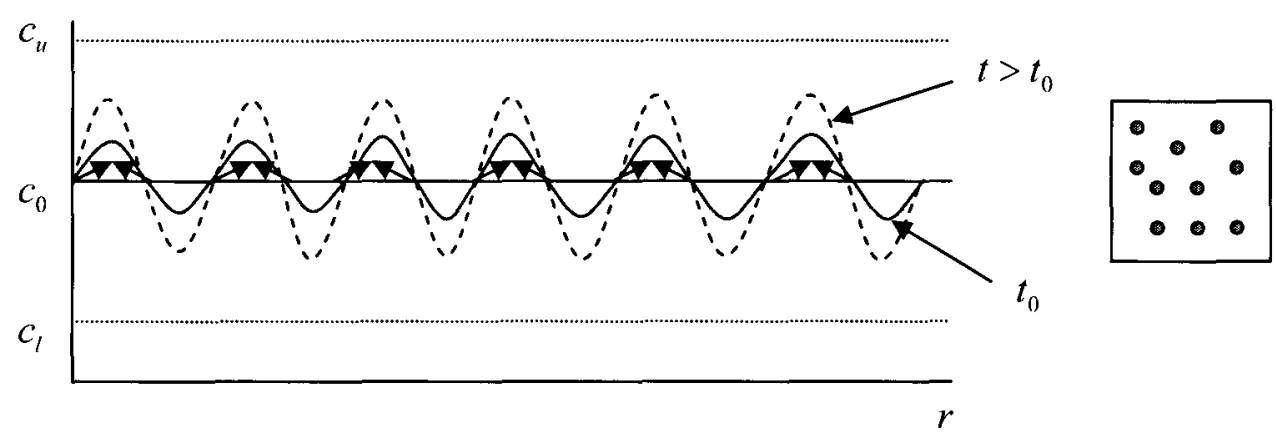

(b)
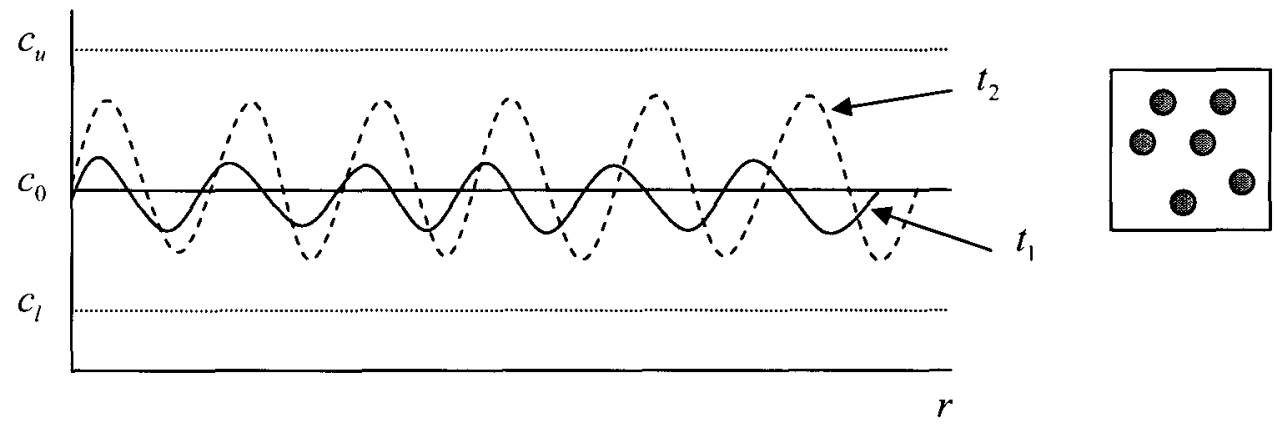

(c)
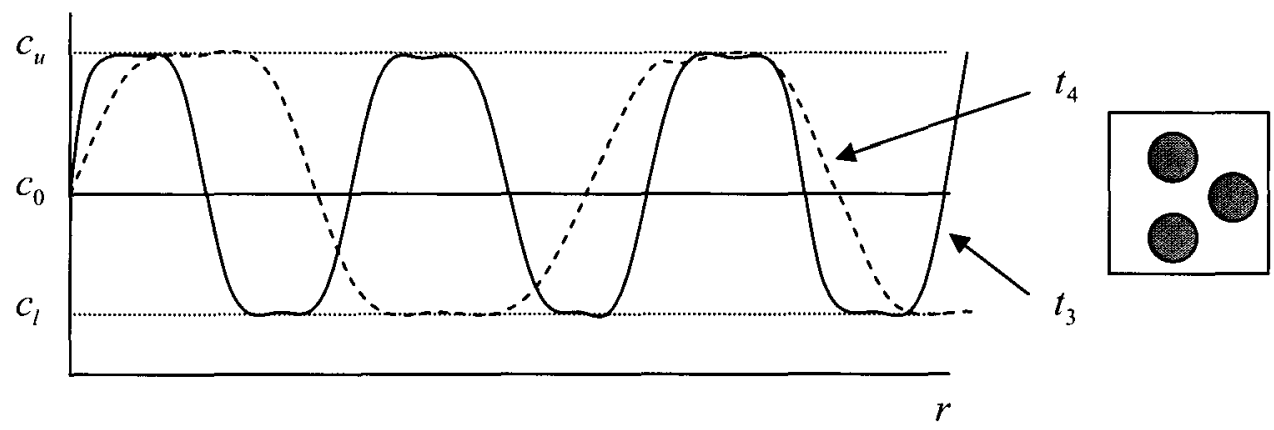

Figure 3.7: The three stages of SD plotted as concentration vs. distance, (a) early stage, (b) intermediate stage and (c) late stage [32]. 
The reduction of solvent from the polymer, migrating towards the droplet, forms the dispersion of droplets within a continuous phase of the polymer. Figures $3.7(a)$ and 3.10 also show the depletion and migration of one the solvent with arrows moving from low concentration to high concentration known as uphill diffusion $[17,34]$. In the intermediate stage, Figure 3.7(b), the concentration fluctuations are still increasing with time from $t_{1}$ to $t_{2}$ showing an increase in amplitude. However, there is an increase in the wavelength of the concentration fluctuation spatially. This effect requires the use of the non-linear $\mathrm{C}-\mathrm{H}$ equation [33]. Finally, in Figure 3.7(c), the late stage of SD, the concentration fluctuations increase until they reach their respective equilibrium concentrations (Figure 3.9 A to B) with constant amplitude, labeled $c_{u}$ (upper concentration) and $c_{l}$ (lower concentration). The wavelength increases with time as the droplets join together to form larger droplets.

\subsubsection{Nucleation and Growth}

Nucleation and growth are associated with metastability, implying the existence of an energy barrier and the occurrence of large composition fluctuations. Nucleation is the process of generating the initial fragments, which are called nuclei, within the metastable region in Figure 3.5. Once these nuclei are formed, the system decomposes with a decrease in free energy, and the nuclei grow. For those nuclei whose sizes are larger than the critical nucleus size, growth occurs rapidly. This growth process and the corresponding phase structure are depicted in Figures 1.4 and 3.8. 


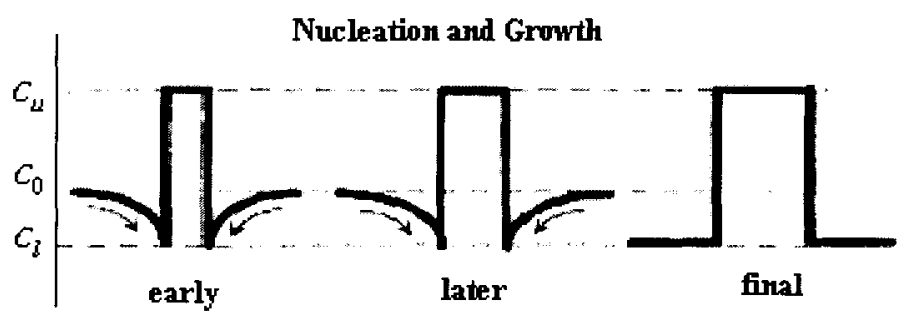

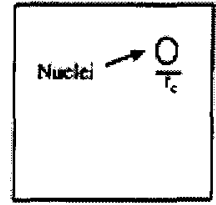

(a)

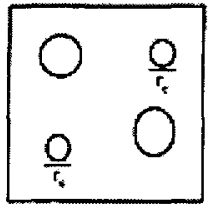

(b)

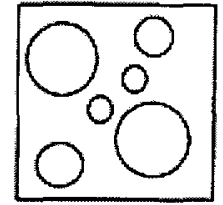

(c)

Figure 3.8: Illustration of the nucleation and growth phase separation mechanism at (a) early, (b) intermediate and (c) final stages. At stage (b) the nuclei form and grow with time leading to the final structure in (c) where many length scales are obtained [34].

As the NG process progresses the concentration of the continuous phase approaches that of the equilibrium composition (Figure $3.9 \mathrm{C}$ to $\mathrm{D}$ ) labeled $c$ (upper concentration) and $c^{\prime}$ (lower concentration). The average size of the nuclei is determined by the kinetics of the process and the amplitude of the fluctuations is determined by the thermodynamic driving force of the system i.e. quench depth. A nucleus possesses an excess surface energy that induces a process forming a new stable phase. This activation energy $\Delta G^{*}$, required to initiate the NG mechanism is expressed as:

$\Delta G^{*}=-\frac{4}{3} \pi r^{3} \Delta G_{t}+4 \pi r^{2} \sigma$

where the first term is the free energy contribution of the nucleus and the second term is the 


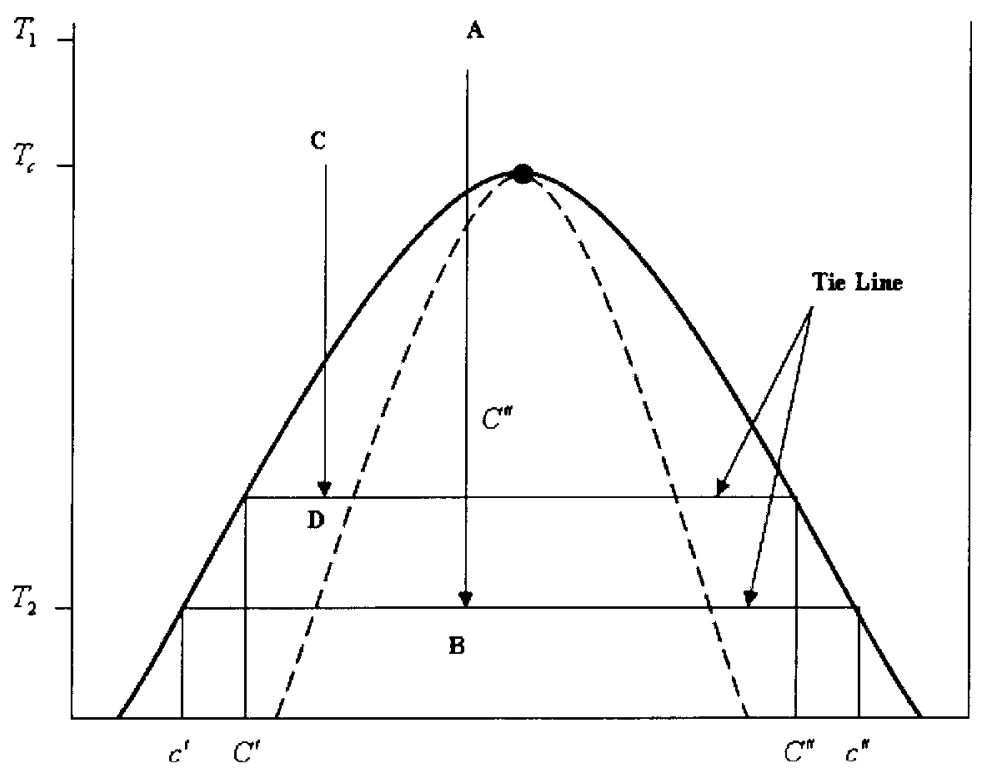

Figure 3.9: Temperature vs. volume fraction phase diagram illustrating the tie lines in TIPS method.

energy required to form an interface between the nucleus and the main phase. $\Delta G_{t}$ is the free energy difference between a nucleus and the main phase while $\sigma$ is the interfacial energy per unit area and $r$ is the radius of the nucleus that can be calculated by minimization of the activation energy with respect to $r$ :

$r=\frac{2 \sigma}{\Delta G_{r}}$

When nuclei form, the system decomposes with a decrease in the free energy causing nucleus growth resulting in droplet domains. 


\subsubsection{Spinodal Decomposition vs. Nucleation and Growth}

Although the equilibrium structure of the $\mathrm{NG}$ and $\mathrm{SD}$ is identical, regardless of the mechanisms of the phase separation; intermediate demixed structures, especially those in the early stage of the phase separation are expected to be quite different for the two mechanisms [125]. The size and amplitude control of the fluctuations in NG mechanism is also opposite of those in SD.

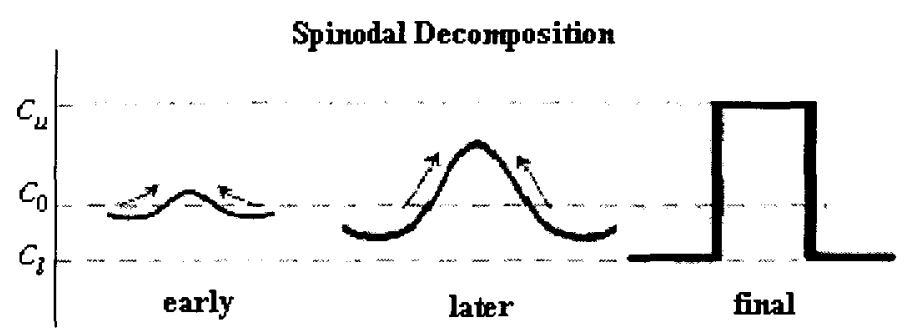

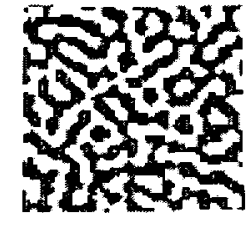

$\vec{\lambda}_{t}$

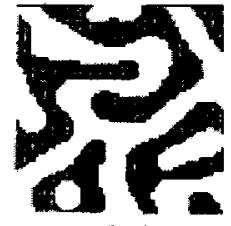

$\overrightarrow{\lambda_{\mathrm{e}}}$

(b)

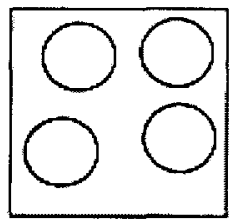

(c)

Figure 3.10: Illustration of the spinodal decomposition phase separation mechanism at (a) early, (b) intermediate and (c) final stages [34].

It has been clearly established that SD process of phase separation does not require activation energy like NG and proceeds spontaneously in the presence of minimal concentration fluctuations or thermal noise. 
In the metastable region, all small fluctuations tend to decay and hence phase separation can proceed only by overcoming the barrier with a large fluctuation in composition. This fluctuation is called a nucleus and once such a nucleus is formed, it grows by a normal diffusion process. The new phase starts forming small nuclei that proceed to grow in size. The molecules that feed the new phase follow the ordinary transport phenomenon by downhill diffusion (Figure 3.8). Inside the spinodal, on the other hand, where the mixture is unstable to infinitesimal fluctuations, there is no thermodynamic barrier to the phase growth, and thus phase separation should occur by a continuous and spontaneous process. Since the solution is initially uniform in composition, this spontaneous reaction must occur by a diffusional flux against the concentration gradient created by small composition fluctuations, that is, by uphill diffusion with a negative diffusion coefficient (Figure 3.10).

In $\mathrm{SD}$, in contrast to $\mathrm{NG}$, there is no sharp interface between the phases until later stages of decomposition. The most significant differences between NG and SD are the mechanisms of decomposition caused by the difference characters of the instability. Even if a solution is homogenous, concentrations are not uniform on microscopic scales. There are always fluctuations about the average concentration $c_{0}$, and these fluctuations lead to a change in the Gibbs free energy. Depending on the curvature, the Gibbs free energy can increase or decrease by the concentration fluctuation. In the metastable region characterized by $\partial^{2} f / \partial c^{2}>0$, the concentration fluctuations cause the increase in the Gibbs free energy and the resulting structure is stable with respect to spontaneous concentration fluctuations. That is why NG is a non activated process and some form of activation mechanism such as nucleation is necessary. In the unstable region characterized by $\partial^{2} f / \partial c^{2}<0$ the fluctuations 
lead to the decrease in the Gibbs free energy and the system becomes unstable even for an infinitesimally small fluctuation. In SD, as already mentioned, phase separation takes place spontaneously and continuously. That is why SD is an activated and irreversible mechanism meaning, once the phase separation has started the system cannot homogenize. During phase separation of NG, the composition of the minor phase domains i.e. nuclei remain constant, and only the size of the domains and their distribution will change with time while in SD a multi-component system of composition $c_{\circ}$ with a concentration fluctuation continuously decompose into two phases of composition. In this case both the composition and size of the domains increase continuously with time. The arrows in Figures 3.8 and 3.10 indicate the direction of diffusion. Therefore, the molecules diffuse from higher concentrations toward lower concentrations in NG which is a positive diffusion (downhill diffusion) and in SD the diffusion coefficient is negative and molecules abnormally diffuse toward higher concentrations from lower concentrations (uphill diffusion).

Experimental results by Kiran and Liu $[93,94]$ show that phase separation by nucleation and growth and spinodal decomposition mechanisms can be differentiated and the binodal and spinodal envelopes can be mapped out using time- and angle-resolved light scattering. For quenches leading to spinodal decomposition, the characteristic wave number $q$ corresponding to the scattered light intensity maximum is observed to be non-stationary in the nearby $q$ range of the instrument, moving to lower wave numbers in time within seconds, suggesting that, in polymer solutions undergoing pressure quench, the early stage of phase separation is limited to extremely short times. Figure 3.11 is a phenomenological description of the time 

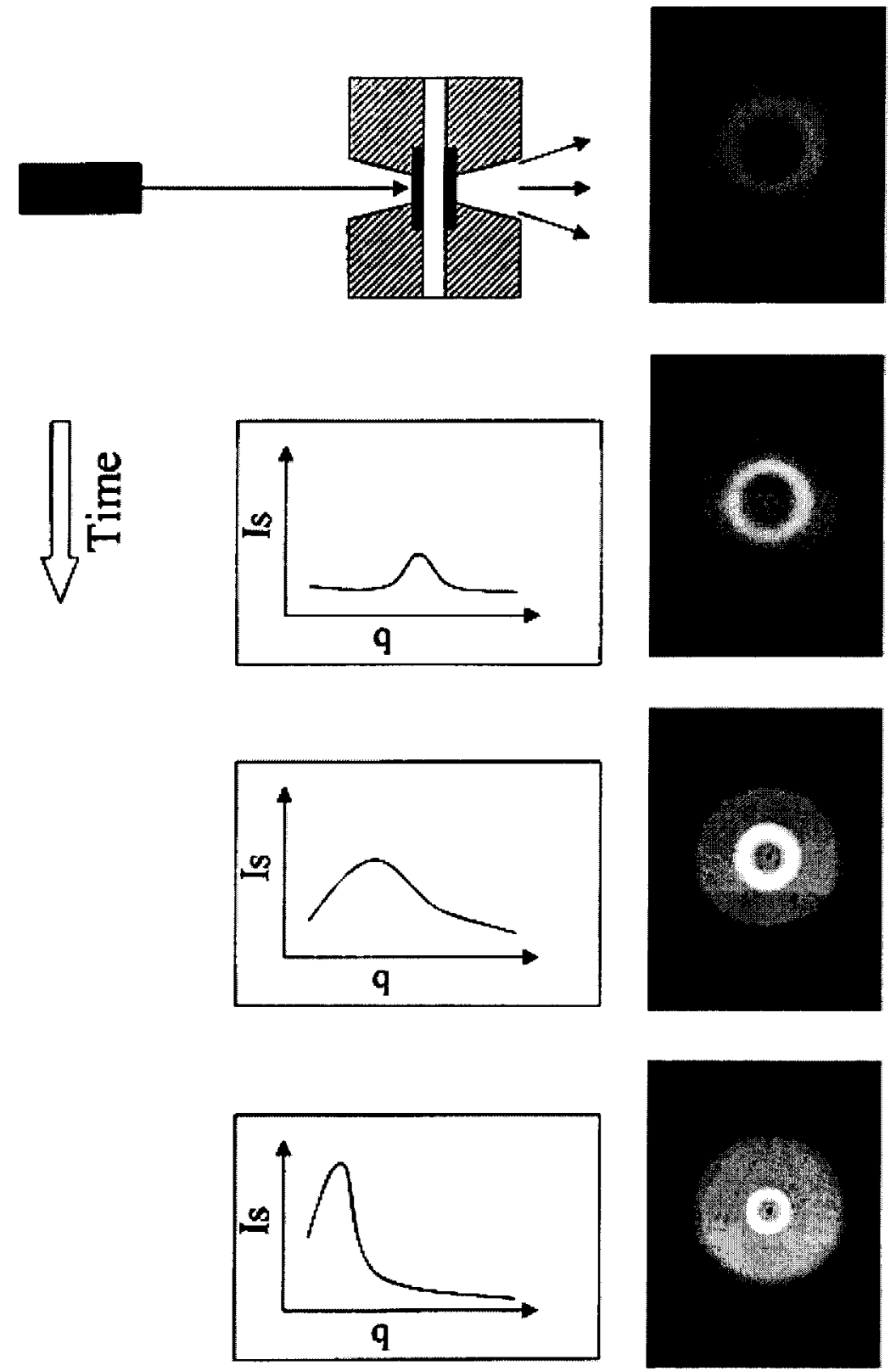

Figure 3.11: Schematic representation of the time-evolution of the scattered light intensities with time and angle (or wave number $q$ ) during spinodal decomposition. The spinodal ring that forms becomes more intense and moves to smaller angles with time. The scattering maximum in the angular dependence corresponds to the location of the ring [93]. 

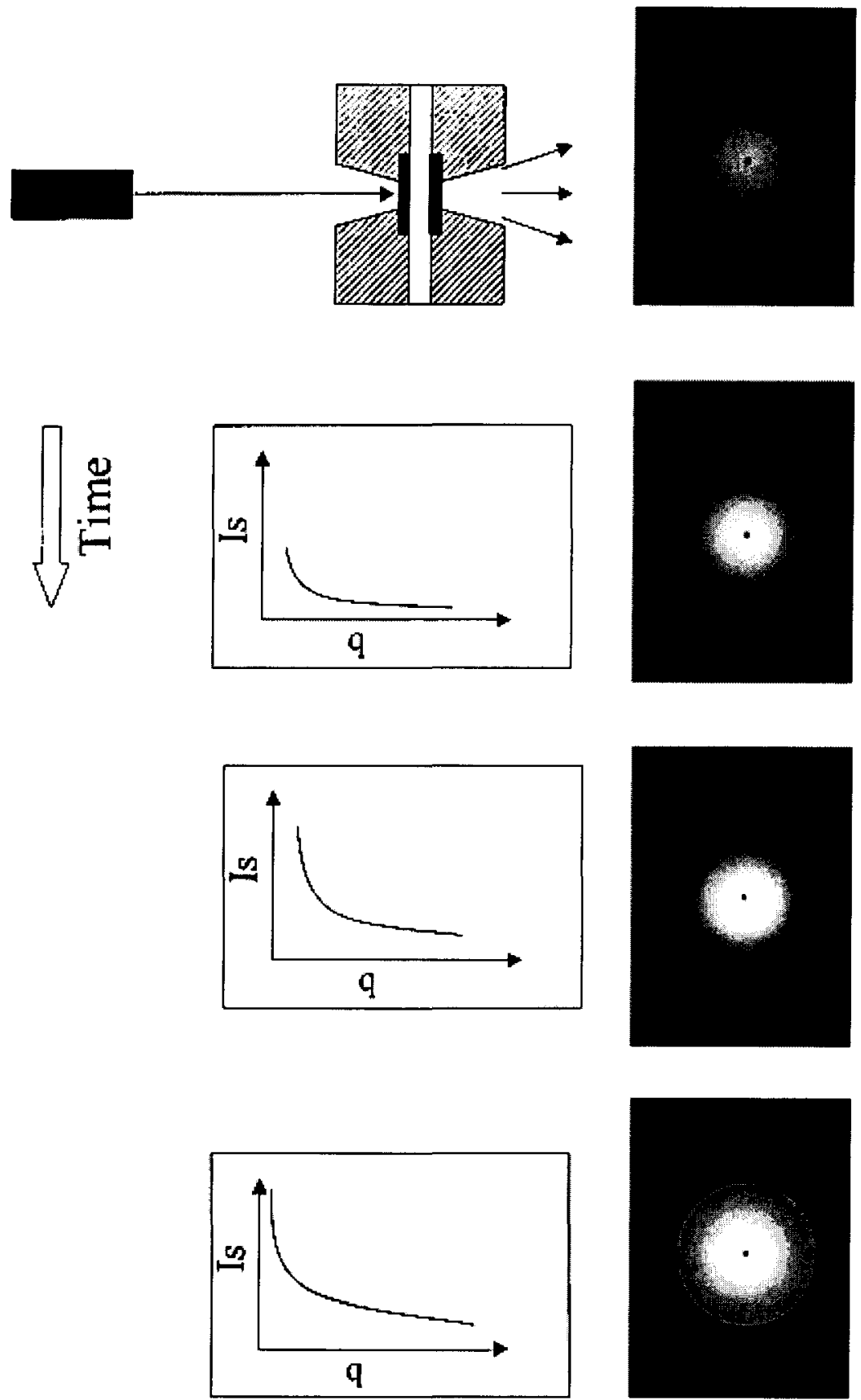

Figure 3.12: Schematic representation of the time-evolution of the scattered light intensities with time and angle (or wave number $q$ ) during nucleation and growth. In contrast to spinodal decomposition, ring formation is not observed and the scattered light intensities do not pass through a maximum [93]. 
evolution of the light scattering pattern in a system undergoing phase separation by spinodal decomposition. The light scattering pattern develops a distinct scattering ring indicative of structure formation in the system. In the later stages of phase separation the ring moves towards the center and eventually collapses. The ring shows itself as a scattering maximum in intensity versus scattering angle $\theta$ or scattering number $[q=4 \pi / \lambda \sin (\theta / 2)]$ plot where $\lambda$ is the wavelength of the laser. With time the maximum in the scattered light intensity moves to lower angles, whereas the scattered light intensity increases [93]. Figure 3.12 shows the fingerprint scattering when the phase separation proceeds with the nucleation and growth mechanism. The scattered light intensity $I$ shows a gradual decrease from low to high angles or wave numbers $q$. The scattered light intensities increase with time at a given angle. No distinct scattering ring is observed in this case.

Both experimental [33-35] and numerical [41-44] results from the TIPS method indicate that the droplets formed by SD mechanism are more homogeneous in size and evenly distributed in the matrix (Figures 1.2 and 3.10) but the ones formed by NG mechanism are almost inhomogeneous droplets that from and grow individually and are randomly positioned (Figures 1.4 and 3.8). As noted in the introduction, the droplet size and distribution are important factors that affect the efficiency of PDLC's as light shutters. The droplet diameter decreases with increasing diffusion, polymer molecular weight and quench depth. Thus, as diffusion increases so does the driving force for phase separation, and a structure of shorter wavelength results. In other words, there is more phase separation. 


\subsubsection{Cahn-Hilliard Theory}

While nucleation theories date back to Becker and Doring [140] in 1935 and later Lifshitz and Slyozov [145] in 1961, the first attempt to treat spinodal decomposition was in 1958 via the phenomenological Cahn-Hilliard equation [1]. Cahn and Hilliard were the first to discuss the spontaneous phase separation of mixtures via spinodal decomposition in binary alloys of metals. This particular process of phase separation does not require activation energy unlike NG mechanism, but proceeds spontaneously in the presence of minimal concentration fluctuations or thermal noise. As already mentioned, SD can be classified by three stages according to time: early, intermediate, and late. The early stage can be characterized by a linearized diffusion equation which has been solved analytically. There is, however, no analytical solution to the intermediate and late stages of SD. Cahn-Hilliard equation is based on the assumption that the total free energy of an inhomogeneous, binary mixture is expressed as sum of two terms:

$$
F=\int\left(f(c)+\kappa\|\nabla c\|^{2}\right) d V
$$

where $c$ is the volume fraction. In this thesis, $c$ is defined as the solvent volume fraction in the binary mixture. $\kappa$ is a positive gradient energy parameter related to the interfacial constant, and $f(c)$ is the free energy of a homogeneous mixture. The first term in equation (3.20) represents the homogeneous free energy, and the second term takes into account any increases in free energy arising from concentration gradients. 
These two terms can be obtained from the Taylor series expansion of a free energy density [42]. In order to describe phase separation in polymer solutions, the model equation can be derived from a continuum model. Considering only diffusional flux $J$, continuity equation can be written as:

$\frac{\partial c}{\partial t}=-\nabla \cdot J$

If we consider only pure diffusion, the net flux $J$, might be expressed as the product of concentration dependent mobility $M$ and the gradient of the chemical potential $\mu$ of each component:

$J=-M N\left(\mu_{2}-\mu_{1}\right)$

Here $M$ is the mobility, which is treated as a constant in the linear theory. The chemical potential is the functional derivative of the free energy:

$\mu_{2}-\mu_{1}=\frac{\delta F}{\delta c}=\frac{\partial f(c)}{\partial c}-2 \kappa \nabla^{2} c$

Combining equations (3.21), (3.22) and (3.23) results in the nonlinear Cahn-Hilliard equation:

$\frac{\partial c}{\partial t}=\nabla \cdot\left[M \nabla\left(\frac{\partial f(c)}{\partial c}-2 \kappa \nabla^{2} c\right)\right]$ 
Cahn linearized this non-linear equation about the average concentration $c_{\circ}$. For very short times following the quench, one would expect this linearization to be valid since the concentration fluctuations should be small.

Therefore, the linear C-H equation would be:

$$
\frac{\partial c}{\partial t}=M\left(\left.\frac{\partial^{2} f(c)}{\partial c^{2}}\right|_{c_{i}} \nabla^{2} c-2 \kappa \nabla^{4} c\right)
$$

$M$ and $\kappa$ are assumed constant. For long wavelength fluctuations in which $\nabla^{4} c$ can be neglected in the above equation, the diffusion equation would be recovered but with a spatially changeable diffusion constant:

$$
D=M\left(\frac{\partial^{2} f(c)}{\partial c^{2}}\right)
$$

Equation (3.26) is known as a collective diffusion coefficient [14]. This diffusion constant is negative inside the spinodal region. For this reason, Cahn termed the initial stage of spinodal decomposition uphill diffusion. Taking the Fourier transform of equation (3.25):

$$
c(\mathbf{r}, t)-c_{\circ}=\sum_{k} A(\mathbf{k}, t) e^{i \mathbf{k} \cdot \mathbf{r}}
$$

where $A(\mathbf{k}, t)$, the magnitude of the Fourier transform of the concentration fluctuations in the system is:

$$
A(\mathbf{k}, t) e^{i \mathbf{k} \cdot \mathbf{r}}=A(\mathbf{k}, 0) e^{R(k) t}
$$


and the growth rate (amplification factor), $R(k)$ is given as:

$R(k)=-M k^{2}\left(\left.\frac{\partial^{2} f(c)}{\partial c^{2}}\right|_{c_{0}}+2 \kappa k^{2}\right)$

where $k_{i}=\frac{2 \pi}{\lambda_{i}}$ and $\lambda_{i}$ is the wavelength for fluctuation $i$. For concentration fluctuations to occur $R(k)$ has to be positive and $\left|\frac{\partial^{2} f(c)}{\partial c^{2}}\right|_{c_{0}}|>| 2 \kappa k^{2} \mid$. Thus inside the classical spinodal region where $\left(\partial^{2} f / \partial c^{2}\right)<0, R(k)$ is positive for $k<k_{c}$ in the unstable region as shown in Figure (3.13). $R(k)$ changes sign at the critical wavenumber:

$k_{c}=\left[\left(-\frac{1}{2 \kappa}\right)\left(\left.\frac{\partial^{2} f(c)}{\partial c^{2}}\right|_{c_{0}}\right)\right]^{1 / 2}$

and has a sharp minimum at:

$k_{m}=\frac{1}{\sqrt{2}} k_{c}$

In equation (3.28) the exponent contains the amplification factor, therefore, the concentration fluctuations that grows the fastest is $k_{m}$ [108]. The above derivation shows that the linear C$\mathrm{H}$ equation predicts phase separation as a superposition of periodic concentration fluctuations of fixed wavelengths with random amplitude, orientation and phase [17]. This model has been used in the prediction of morphology for TIPS and polymerization induced phase 
separation (PIPS) [17]. In scattering experiments, however, $c(r, t)$ is not measured but instead, the structure factor which is proportional to scattering intensity is measured by:

$$
I(\mathbf{k}, t)=I(\mathbf{k}, 0) e^{2 R(k) t}
$$

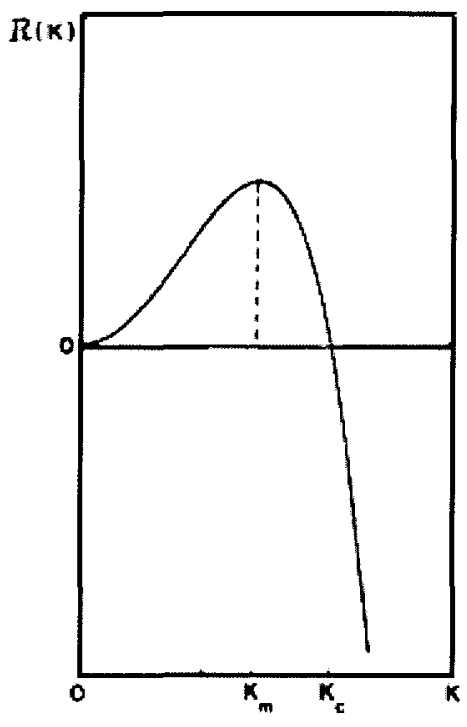

Figure 3.13: Growth rate $R(k)$ vs. the wavevector $k$. Following the linear theory, $R(k)$ is positive for $k<k_{c}$ and exhibits a maximum at $k=k_{m}$ [108].

where $I(k, 0)$ is the initial intensity. Thus according to the linear theory the initial stages of SD should produce an exponential growth in intensity for $k<k_{c}$, with a peak at time dependent wavenumber $k_{m}$. Cahn interpreted this $k_{m}$ as the wavenumber which characterizes the fine uniformly dispersed precipitate seen in SD studies [115]. Quantitative information can be obtained from the time-resolved light scattering intensity profile $l(q, t)$, where $q$ is the scattering wavevector. 
The numerical equivalence of this profile is the structure factor $S(\mathbf{k}, t)[120]$, i.e. $I(q, t) \propto S(\mathbf{k}, t)=\left\|A(\mathbf{k}, t)^{2}\right\|$ for $q=\mathbf{k}$. Consequently, the light intensity $I(q, t)$ contains information on the concentration fluctuation time and length scales. Figure 3.14 shows a sharp peak existing at time $t_{1}$ which gradually decreases in sharpness with increasing time to $t_{2}$ and finally at $t_{3}$, the peak decreases further and increases in width. This is representative of what occurs in SD. The change in the intensity (shifting of the height and position of the peaks) as time changes indicates the changes in the coarsening of the phase separating structure for the intermediate and late stages of SD. The relation is shown in equations (3.33) and (3.34) for $q=\mathbf{k}$. The first is the scattering intensity, which is now defined as:

$I(q, t)=I(q, 0) e^{2 R(q) t}$

$I(q, t)$ is the scattering intensity, $q$ is the scattering vector, $R$ an amplification factor and $t$ is time. The scattering vector $q$, is a function of the wavelength $\lambda$, and the scattering angle $\theta$, expressed as:

$$
q=\left(\frac{4 \pi}{\lambda}\right) \sin \left(\frac{\theta}{2}\right)
$$

The wavelength $\lambda$ is obtained from equation (3.25) and is expressed as:

$$
\lambda=2 \sqrt{2} \pi\left[\left(-\frac{1}{2 \kappa}\right)\left(\left.\frac{\partial^{2} f(c)}{\partial c^{2}}\right|_{c_{0}}\right)\right]^{1 / 2}
$$




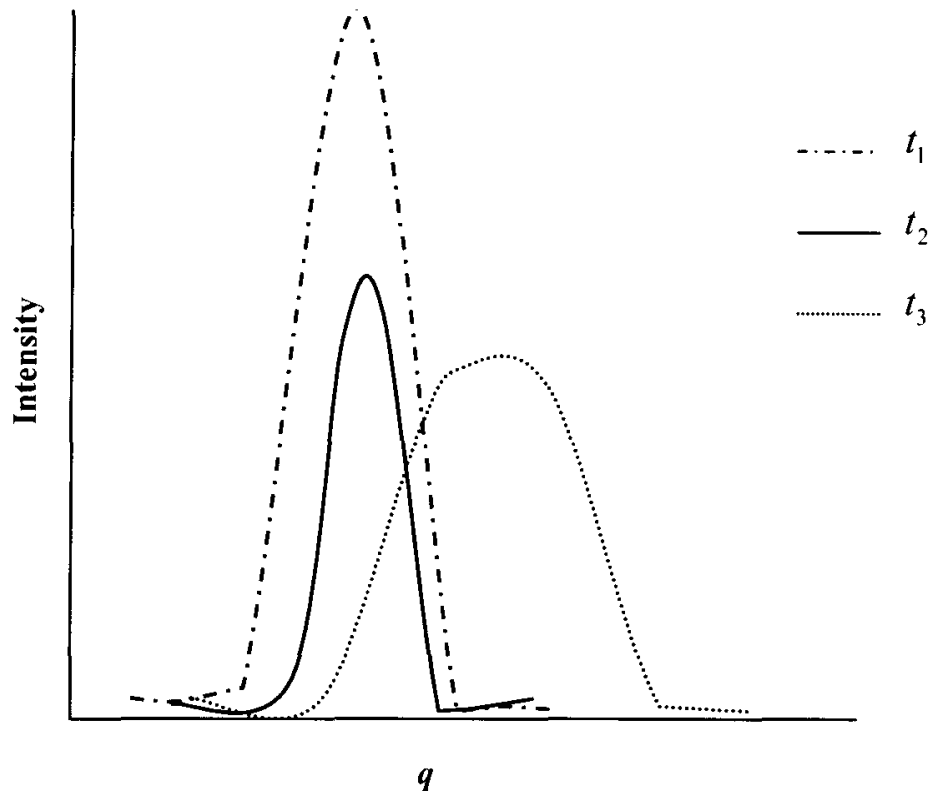

Figure 3.14: A typical plot of the intensity $I(q, t)$ vs. scattering vector $q$, for studying phase separation by SD with time.

The morphological features study, such as the phase structure type and droplet size distribution, is the most important aspect of the TIPS process. Depending on initial average concentration, two different types of morphology are formed: the interconnected structure morphology and the droplet-type morphology. Therefore, equation (3.35) is a key methodology to predict the morphological features of polymer solutions in off critical TIPS. The predicted droplet diameter $\left(d_{m} \propto \lambda / 2\right)$ can be calculated in advance so that the desired and required phase structure can be obtained at an initial concentration. Figure 3.15 schematically shows relationship between the droplet diameter $d m$ and the wavelength $\lambda$. 


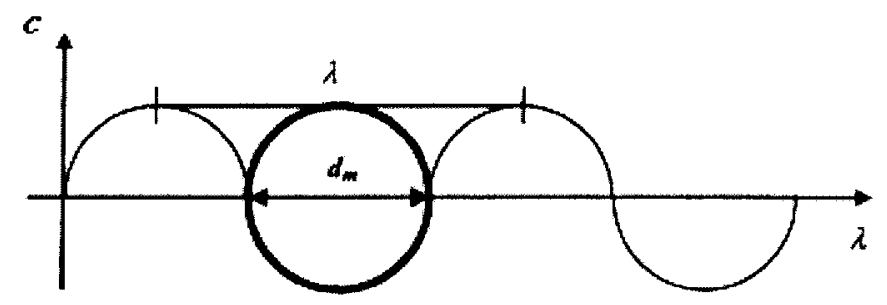

Figure 3.15: Schematic representation of the relationship for droplet diameter $d_{m}$ and wavelength $\lambda$ in two dimensions. $c$ is the average concentration. The circle represents a polymer droplet, since the droplet diameter equals half of the wavelength $d_{m} \propto \lambda / 2$ [120].

\subsubsection{Noise Term}

Cook [149] made an important contribution to the theoretical development of the linear theory by observing that it is necessary to add a noise term $\eta$, to C-H equation (3.21) in order to have a correct statistical description of the dynamics. Cook's observation was that, in addition to a flux produced by the gradient of a local chemical potential, there is additional flux arising from random thermal motion of the atoms:

$\frac{\partial c}{\partial t}=-\nabla \cdot J+\eta$

where $\eta$ is the random force term taken to be a Gaussian distribution. Its mean value is zero and the correlation satisfies the fluctuation-dissipation relation [150], i.e.: $\langle\eta\rangle=0$. Cook's noise term, however, is neglected from the current study. This term has been found to have 
little effect on the domain growth of simple fluids and in off-critical quench conditions. It is often omitted for numerical studies which cover the areas far from critical point. Novick [160] in his numerical work verified he behavior of the nonlinear Cahn-Hilliard equation for asymmetric systems within the unstable spinodal region. He tried to demonstrate those features of spinodal decomposition and nucleation which are obtainable by study of the nonlinear Cahn-Hilliard equation without reference to a specific noise source and demonstrated that while the Cahn-Hilliard equation cannot be considered to contain all the information contained in a full noise driven approximation the theory is considerably richer than what would be predicted from linear theory only. Oono and Puri [173] computationally modeled space-time phase ordering dynamics of unstable SD region. The two dimensional lattice of $100 \times 100$ was used for the simulation. There, they supported the idea that the noise effect is unimportant for the late stages of phase separation kinetics [173]. In another simulation performed by them, the long-time behavior of two-dimensional systems undergoing spinodal decomposition was studied numerically with the aid of a cell-dynamical approach both without and with noise [174]. In both cases, the representative length scale of the pattern behaved the same where the crossover time increased with an increase in amplitude of the noise. They demonstrated that the effect of noise appears to be unimportant. They also performed simulations in which noise had a Gaussian distribution and this made no difference to their results. Furthermore, they also studied the evolution patterns in the case where they started off with zero (non-zero) amplitude of noise and switched on (off) the noise after a certain number of iterations. The patterns rapidly (within about 10 iterations) went to the noisy (noiseless) forms. This indicated that there is no cumulative effect of noise. 
In the usual interpretation of the deterministic C-H-C equation they even simply discarded the noise while retaining the usual kinetic coefficients.

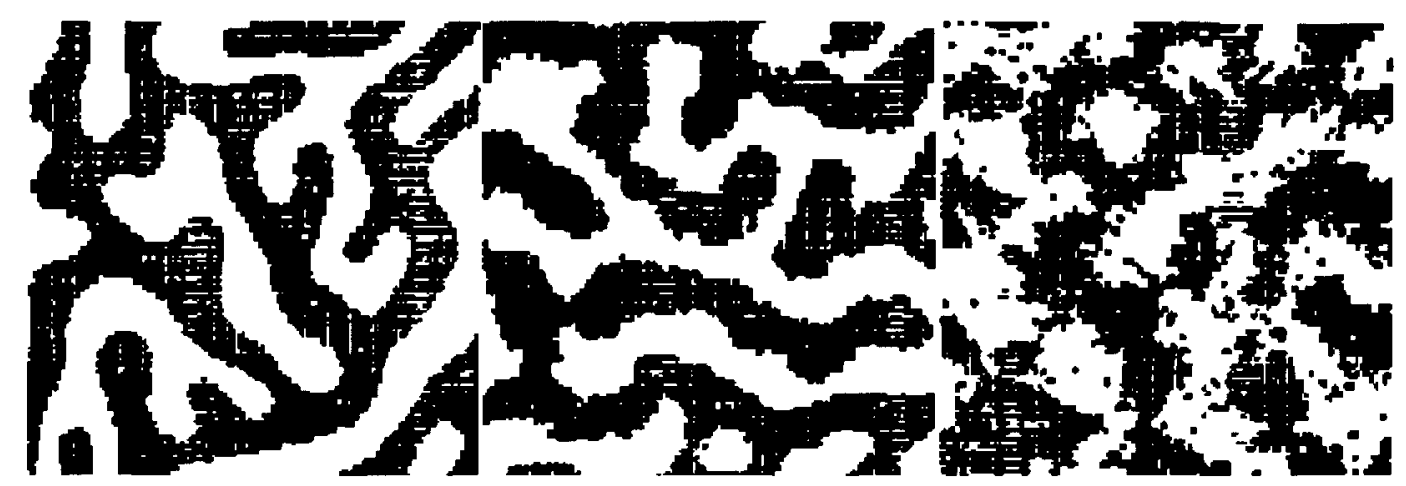

(a)

(b)

(c)

Figure 3.16: Typical pattern for the noiseless case (a), noisy case (b) and strongly noisy (c) [174].

Figure 3.16 shows the patterns obtained from the same initial conditions for the noiseless and noisy cases. For the noiseless case (a) the boundary walls are smooth and regular. In the noisy (b) case the pattern size at comparable times is of the same order as in the noiseless case, but the boundary walls are ragged. In case (c) a typical pattern for the strongly noisy case with the same initial conditions as before is shown. This pattern is more ragged than the pattern for the case (b) and is similar to previously published patterns [174] from Monte Carlo simulations. They, as well, plotted the scaled scattering function $S(k, t)(\langle k\rangle(t))^{2}$ as a function of $k /\langle k\rangle(t)$ for different times. In the scaling regime a universal curve was expected. Figure 3.17 shows data from different times for the noiseless case (denoted by circles). 
They can be seen to lie on a smooth master curve. The points marked by tiny crosses in Figure 3.17 correspond to the noisy case. Both the noiseless and noisy cases have the same master curve for moderate values of $k /\langle k\rangle(t)$. However, the tails of the curves, which correspond to relatively (compared to pattern size) short wavelength fluctuations, are quite different though this difference is not evident on the scale of the figure. For the noiseless case, the tail of the curve drops off faster than $x^{-3}$, where $x=k /\langle k\rangle(t)$. The same model as Oono and Puris' $[173,174]$ without noise was studied extensively by Chakrabarti and Gunton later.

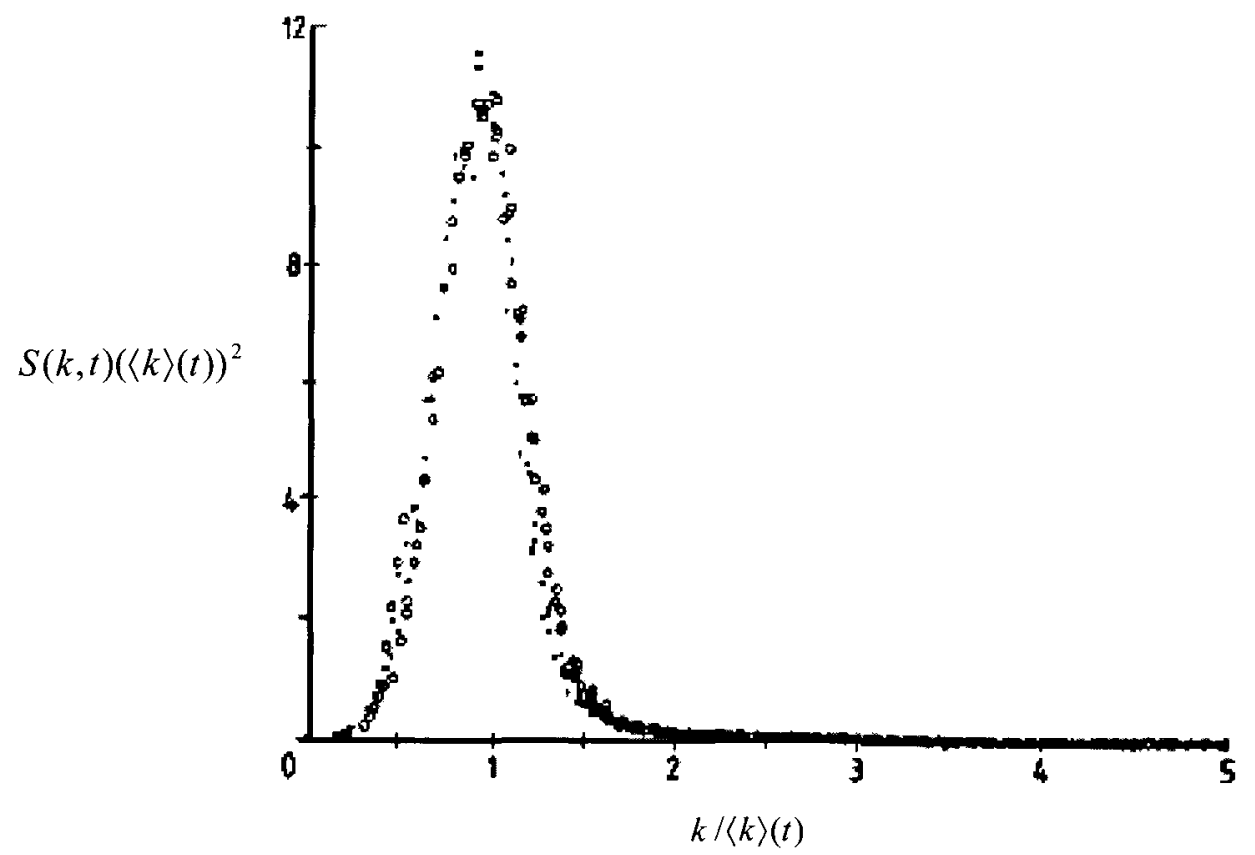

Figure 3.17: Scaled scattering function $S(k, t)(\langle k\rangle(t))^{2}$ as a function of $k /\langle k\rangle(t)$ for the noiseless and noisy cases. The circles (tiny crosses) are data from the noiseless (noisy) case at different times. Though it is not evident on the scale of this figure, for the noiseless case, the tail of the curve drops off faster than $x^{-3}$. 
In a study performed by Yeung [178] over the dynamics governed by the time-dependent Ginzburg-Landau and Cahn-Hilliard equation, the results were consistent with numerical simulations which confirmed that the scaling function is independent of the magnitude of the noise. Chakrabarti and Brown $[71,72]$ demonstrated in contrary to the Ginzberg-Landau model that suggests the noise effects are irrelevant for the late time growth laws and scaling behavior in small molecule systems [189] when noise effects are included, the thermally induced fluctuations in the local concentration are important in late time domain growth for the polymer solutions but however, both off critical and critical mixtures behave similarly for deep quenches (which is a benefit to our assumption of ignoring the noise term in the simulation). Zhu et al. [175] implemented semi-implicit Fourier spectral method is to solve the Cahn-Hilliard equation with a variable mobility. The method is more efficient than the conventional forward Euler finite-difference method, thus allowing them to simulate large systems for longer times. They studied the coarsening kinetics of interconnected two-phase mixtures using a Cahn-Hilliard equation with its mobility depending on local compositions. To simplify the problem, they did not include the noise term in their study because it usually took a lot of CPU time for generating the Gaussian noise. The noise term has no significant effect on the time to get scaling results or the stability of the numerical algorithm, they believed. For bulk-diffusion-controlled dynamics, it is usually accepted that the noise term does not affect some important features of the late stages of evolution such as the growth law for the characteristic domain size and the scaling functions for spinodal decomposition. However, Yeung et al. [178] believed that noise might be more important in interface diffusion-controlled dynamics. Noise may affect the mobility, leading to a faster rate of growth. The effect of the noise term on the kinetics of coarsening through both bulk diffusion 
and interface diffusion remains a topic for researchers to carry out large scale and long time simulations. Clarke [55] independently investigated a process for obtaining controlled morphologies in polymer blends and modeled it numerically. Particles of one type of polymer were allowed to dissolve in a matrix of a dissimilar polymer. Prior to complete dissolution the blend was quenched into the two-phase region, such that phase separation took place. The noise term in his simulation has also been neglected. The inclusion of the noise term greatly increases the computation time and is believed not to affect the general behavior he admitted. Fialkowski and Holyst [83] analyzed a two-step process of phase separation for binary mixtures. The system was first quenched into the thermodynamical instability region where the mixture undergoes a process of spinodal decomposition. Next, they heated up the system to make a temperature jump back to the curing temperature above the spinodal. There, they assumed a fully deterministic dynamic without the thermal noise in the system. In this thesis however, we have ignored the noise term for simplicity of the simulated model and decreasing the computational time based on the experimental and numerical studies that have been so far performed on its effects.

\subsubsection{Concentration Dependent Mobility and Mutual Diffusion}

The kinetics of phase separation by SD can best be described by the Cahn-Hillard equation (C-H). The $\mathrm{C}-\mathrm{H}$ equation describes the fluctuations in concentration in terms of wavelengths for the three stages of SD. The early stage of SD can be described by the linear C-H equation. 
The intermediate and later stages require the non-linear C-H equation. The difficulty in modeling polymer solutions is determining the constants in $\mathrm{C}-\mathrm{H}$ equation such as mobility, F-H interaction parameter and the constant related to the interfacial constant. However, there have been several techniques used to determine these parameters for polymer solutions [3572]. The mobility in the $\mathrm{C}-\mathrm{H}$ equation is assumed constant but has been shown to be dependent on concentration and temperature [36]. The mobility can be written in the form of:

$$
M(c)=\frac{D(c)}{\frac{\partial^{2} f(c)}{\partial c^{2}}}
$$

The mobility $M$ and interfacial parameter $\kappa$ are dependent on both the polymer molecular chain length and local concentration. Many numerical studies (including this paper) however, have been performed assuming $M$ and $\kappa$ constant with an attempt to simplify the problems [43-45] and only a few studies have been performed with a molecular weight and concentration dependent mobility [48-50]. The interfacial parameter $\kappa$ can be assumed to be concentration independent in the absence of any accurate information about its behavior, however, $M$ is known to be highly sensitive to concentration changes [47]. The molecular chain lengths and local concentration dependence of the mobility can be captured from its relation with the self diffusion coefficient by equation (3.26). The mutual diffusion coefficient $\Lambda$, for a binary system, is generally given by:

$$
\Lambda=\frac{\Lambda_{1} \Lambda_{2}}{\Lambda_{1}+\Lambda_{2}}
$$


where $\Lambda_{i}$ of each component can be obtained as:

$$
\Lambda_{i}=k_{B} T M_{i}
$$

where $k_{B}$ is the Boltzmann's constant and $T$ is an absolute temperature. Replacing equation (3.39) in (3.38), considering a binary mixture would result in:

$\frac{1}{M}=\frac{1}{M_{1}}+\frac{1}{M_{2}}$

where $M$ is the total mobility and $M_{1}$ and $M_{2}$ are the individual mobility of two components. The total mobility $M$ can be also expressed as:

$$
M=\frac{c v(1-c)}{\xi}
$$

where $c$ is the average concentration of the solution, $v$ is the volume of a cell or segment and $\xi$ is monomer friction coefficient. Equation (3.41) is obtained from combining equations (3.37), (3.40) and the self-diffusion coefficient equation of Rouse model:

$$
D_{i}=\frac{k_{B} T}{N_{i} \xi_{i}}
$$

where $N_{i}$ is the degree of polymerization for each component. 


\section{Chapter 4}

\section{Numerical Method of Solution}

This chapter deals with a computational study of TIPS on the basis of a one and twodimensional mathematical model that describes the TIPS phenomenon of a model binary polymer solution (PDLC) exposed to an externally controlled single and double temperature quench. During the model development, the nonlinear Cahn-Hilliard (CH) theory was used to describe the dynamic behavior of phase separation via NG and SD, and the Flory-Huggins free-energy expression to describe the thermodynamic behavior of the model polymer solution.

The emphasis in this thesis is placed on understanding the influence of a double temperature quench on the formation of cavity structures during the double quench TIPS process. The rest of this thesis is focused on the development of the model, presenting the governing equations, initial and boundary conditions, and numerical methods. The numerical results are

presented and discussed in chapter 5, and finally conclusions and recommendations are drawn in chapter 6 . 


\subsection{Model Development}

The nonlinear $\mathrm{C}-\mathrm{H}$ equation [1] was originally developed from the continuity equation of mass for metals, and was later extended to polymer blends by deGennes [50]. The continuity equation may be expressed as:

$$
\frac{\partial c}{\partial t}=-\nabla \cdot J
$$

where $c$ is the concentration (taken as volume fraction in this thesis) of one of the polymer components, and $J$ is the interdiffusional flux of the components. $J$ is related to the gradient in chemical potential through:

$$
J=-M \nabla\left(\mu_{2}-\mu_{1}\right)=-M \nabla \frac{\delta F}{\delta c}
$$

In the derivation of the $\mathrm{C}-\mathrm{H}$ equation the mobility $M$ is assumed to be constant. However, the concentration dependence of the mobility for polymer solutions is considered. $\mu_{1}$ and $\mu_{2}$ are the chemical potentials of the polymer and solvent, respectively. The total free energy $F$ is expressed as:

$$
F=\int\left(f(c)+\kappa\|\nabla c\|^{2}\right) d V
$$

where $f(c)$ is the free energy density of the homogeneous polymer mixture and $\kappa\|\nabla c\|^{2}$ takes into account the increase in free energy from the concentration fluctuations. 
In the derivation of the $\mathrm{C}-\mathrm{H}$ equation the concentration dependence of $J$ for polymer solutions is taken into account as well. An essential ingredient of a nonlinear modification of the $\mathrm{C}-\mathrm{H}$ equation is the selection of an appropriate expression for the bulk free energy density, and a proper choice in polymer studies is the Flory-Huggins (F-H) free energy. The F-H free energy equation $[112,113]$ is derived from a combinatorial lattice theory, and is used to describe polymer solution thermodynamics. The F-H equation can be adapted for simple binary solutions (i.e., small molecule solutions) by setting the degrees of polymerization to unity and the interaction parameter term to zero. Consequently, the remaining terms form the so-called strictly regular solution theory. The F-H equation can also be used for polymer blends, because it can take into account the degrees of polymerization of both polymers. For instance, Brown et al. $[121,122]$ and Chakrabarti et al. $[71,72]$ used this equation in their simulations of phase separation in polymer blends. The F$\mathrm{H}$ equation, however, does have its limitations; nevertheless, it is quite successful in phase equilibrium studies [124]. The homogeneous free energy term that can be obtained from the Flory-Huggins theory [104] is:

$$
f(c)=\frac{k_{B} T}{v}\left[\frac{c}{N_{1}} \ln c+\frac{(1-c)}{N_{2}} \ln (1-c)+\chi c(1-c)\right]
$$

where $\chi$ is the interaction parameter, $v$ is the volume of a polymer segment (i.e., monomer), $k_{B}$ is Boltzmann's constant, $T$ is temperature, and $N_{1}$ and $N_{2}$ are the degrees of polymerization of solvent and solute (polymer), respectively. The nonlinear $\mathrm{C}-\mathrm{H}$ equation is then obtained by combining equations (4.1) and (4.4) for two-dimensional analysis: 
$\frac{\partial c}{\partial t}=\nabla M \nabla\left(\frac{\partial f(c)}{\partial c}\right)+M \nabla^{2}\left(\frac{\partial f(c)}{\partial c}\right)-2 \kappa \nabla M \nabla^{3} c-2 \kappa M \nabla^{4} c$

The fast mode and slow mode theories are used to describe the mobility of polymers upon mixing [160]. The fast mode theory predicts that the diffusion rate is limited by the faster component, while the slow mode theory predicts that mutual diffusion of the binary polymer solution is limited by the slower component in the mixture. The slow mode theory is used in this thesis since it better reflects the slow diffusion process in viscous polymer solutions. The slow mode theory states that the mobility is related to the self-mobility of the individual components $M_{1}$ and $M_{2}$ :

$M=\frac{M_{1} M_{2}}{M_{1}+M_{2}}=\frac{c v(1-c)}{\xi}$

The individual mobility of each component are expressed in terms of a self-diffusion coefficient:

$D_{i}=M_{i} \frac{\partial^{2} f(c)}{\partial c_{i}{ }^{2}} \quad$ for $i=1,2$

where $c_{i}$ is the concentration of component $i$, so that $c_{1}+c_{2}=1$ and $c_{1}=c$ then $c_{2}=1-c$. The self-diffusion coefficients measure the rate at which individual components of the mixture diffuse. The self-diffusion coefficient can be described by:

$D_{i}=\frac{k_{B} T}{N_{i} \xi_{i}}$ 
where $N_{i}$ is the degree of polymerization, and $\xi_{i}$ is the monomer friction coefficient of component $i$. For polymer solutions, the degree of polymerization of the solvent is taken as unity; that is $N_{1}=1$. Flory's interaction parameter $\chi$ is related to the temperature via the theta temperature $\theta$ and dimensionless entropy of dilution parameter $\psi$ :

$\chi=\frac{1}{2}-\psi\left(1-\frac{\theta}{T}\right)$

The structure factor is an important parameter that is typically used to characterize the development and evolution of the morphology during SD and relates numerical results with experimental findings. The structure factor is computed using the fast Fourier transform of the computed concentration values [118]. The relationship between experimental results (the intensity from light scattering experiments) and numerical results (the structure factor) is of the following form:

$$
I(q, t)=S(\mathbf{k}, t) \quad \text { for } q=\mathbf{k}
$$

\subsection{Governing Equation}

To develop a model for two-dimensional two-step TIPS phenomenon by SD in an asymmetric polymer solution, following assumptions have been made. This model was programmed and run through SHARCNET (a consortium of Canadian academic institutions 
who share a network of high performance computers) [188], which helps solve highly complex problems, perform heavy numerical analyses, or to run computationally intensive workloads that are in scale far beyond the tasks that could be achieved on today's leading desktop systems. The following conditions and assumptions are made in the model developed in this thesis:

1. Entanglement properties are considered to be identical $\left(\xi_{1}=\xi_{2}=\xi\right)$.

2. Interaction parameter $(\chi)$ is a function of temperature only.

3. Noise term $(\eta)$ is neglected.

4. Mobility $(M)$ is constant.

5. Gradient energy parameter $(\kappa)$ is a positive constant.

The following two-dimensional fourth-order partial differential equation governing the phase separation phenomenon via SD is obtained after substituting equations (4.4) and (4.6) into equation (4.5):

$$
\begin{aligned}
& \frac{\partial c}{\partial t}=\frac{k_{B} T}{\xi}\left[-\frac{1}{N_{1}}+\frac{1}{N_{2}}-2 \chi(1-2 c)\right]\left[\frac{\partial c}{\partial x} \frac{\partial c}{\partial x}+\frac{\partial c}{\partial y} \frac{\partial c}{\partial y}\right] \\
& +\frac{k_{B} T}{\xi}\left[\frac{(1-c)}{N_{1}}+\frac{c}{N_{2}}-2 \chi c(1-c)\right]\left(\frac{\partial^{2} c}{\partial x^{2}}+\frac{\partial^{2} c}{\partial y^{2}}\right) \\
& -\frac{2 \kappa v}{\xi}(1-2 c)\left\{\left(\frac{\partial c}{\partial x} \frac{\partial^{3} c}{\partial x^{3}}+\frac{\partial c}{\partial x} \frac{\partial^{3} c}{\partial x \partial y^{2}}\right)+\left(\frac{\partial c}{\partial y} \frac{\partial^{3} c}{\partial x^{2} \partial y}+\frac{\partial c}{\partial y} \frac{\partial^{3} c}{\partial y^{3}}\right)\right\} \\
& -\frac{2 \kappa v}{\xi} c(1-c)\left(\frac{\partial^{4} c}{\partial x^{4}}+2 \frac{\partial^{4} c}{\partial x^{2} \partial y^{2}}+\frac{\partial^{4} c}{\partial y^{4}}\right)
\end{aligned}
$$


The following scaling relations along with parameters of Appendix B were used to nondimensionalize the governing and auxiliary (i.e., the initial and boundary conditions) equations for two-dimensional model development:

Dimensionless concentration: $\vec{c}^{*}=c$

Dimensionless space in $x$ direction: $x^{*}=\frac{x}{L}$

Dimensionless space in $y$ direction: $y^{*}=\frac{y}{L}$

Dimensionless temperature: $T^{*}=\frac{T}{\theta}$

Dimensionless time: $t^{*}=\frac{2 v K t}{\xi L^{4}}$

Dimensionless diffusion coefficient: $D^{*}=\frac{k_{B} L^{2} \theta}{2 v \kappa}$

Superscript asterisks denote dimensionless variables. In terms of these dimensionless quantities, the governing equation can be then written as:

$$
\begin{aligned}
& \frac{\partial c^{*}}{\partial t^{*}}=D^{*} T^{*}\left[-\frac{1}{N_{1}}+\frac{1}{N_{2}}-2 \chi\left(1-2 c^{*}\right)\right]\left[\frac{\partial c^{*}}{\partial x^{*}} \frac{\partial c^{*}}{\partial x^{*}}+\frac{\partial c^{*}}{\partial y^{*}} \frac{\partial c^{*}}{\partial y^{*}}\right] \\
& +D^{*} T^{*}\left[\frac{\left(1-c^{*}\right)}{N_{1}}+\frac{c^{*}}{N_{2}}-2 \chi c^{*}\left(1-c^{*}\right)\right]\left(\frac{\partial^{2} c^{*}}{\partial x^{* 2}}+\frac{\partial^{2} c^{*}}{\partial y^{* 2}}\right) \\
& -\left(1-2 c^{*}\right)\left\{\frac{\partial c^{*}}{\partial x^{*}}\left(\frac{\partial^{3} c^{*}}{\partial x^{* 3}}+\frac{\partial^{3} c^{*}}{\partial x^{*} \partial y^{* 2}}\right)+\frac{\partial c^{*}}{\partial y^{*}}\left(\frac{\partial^{3} c^{*}}{\partial x^{* 2} \partial y^{*}}+\frac{\partial^{3} c^{*}}{\partial y^{* 3}}\right)\right\} \\
& -c^{*}\left(1-c^{*}\right)\left(\frac{\partial^{4} c^{*}}{\partial x^{* 4}}+2 \frac{\partial^{4} c^{*}}{\partial x^{* 2} \partial y^{* 2}}+\frac{\partial^{4} c^{*}}{\partial y^{* 4}}\right)
\end{aligned}
$$




\subsection{Initial and Boundary Conditions}

An appropriate initial condition for equation (4.12) is the one that describes the infinitesimal thermal concentration fluctuations that exist initially in the homogeneous phase of the binary polymer solution at thermal equilibrium. Thus far, the most frequent and popular choice of boundary conditions are of periodic type $[14,19]$, which lead to ideal pattern formation [38]. These boundary conditions are used to avoid surface effects [26], and to simplify the computational scheme. The periodic boundary conditions are only appropriate if the computational cell represents a sample of infinite dimensions, which in reality is never true because samples are finite in size and have bounding surfaces through which various exchanges with the environment may occur. Therefore, to model more realistic SD phase separation and pattern formation phenomena, non-periodic boundary conditions are needed. Since there is no mass flow normally through the surfaces, the zero mass flux criteria is an appropriate boundary condition $[18,37]$. In addition, the natural boundary conditions obtained from the variation of the free energy provide another complementary set of boundary conditions for the numerical solution of the nonlinear $\mathrm{C}-\mathrm{H}$ equation [18,37]. The detailed development of this initial and boundary condition is given by Chan and Rey [32] who used Monte Carlo simulations including the linearization approximation and the equipartition theorem. Another improvement that may lead to more realistic simulation results for polymer solutions is the use of asymmetric phase diagrams instead of symmetric ones. The phase diagrams used by Hashimoto et al. [90,91] and Clarke [53-55] were symmetric and those by Inoue [52] and Tanaka [86,87] were nearly symmetric ones. In 
reality, however, binary phase diagrams of polymer or oligomer solutions are generally not symmetric. The dimensionless expression of this initial concentration is written as:

$c^{*}\left(t^{*}=0\right)=c_{0}^{*}+\delta c^{*}\left(t^{*}=0\right)$

where $c_{0}^{*}$ is the dimensionless (initial) average concentration and $\delta c^{*}\left(t^{*}=0\right)$ represents the initial concentration fluctuations (at thermal equilibrium). Additionally, two non-periodic boundary conditions are used to solve the governing equation. The natural boundary conditions, which are obtained from variational analysis, are expressed as following in dimensionless form for a two-dimensional model:

\begin{tabular}{|c|c|c|c|c|c|c|}
\hline$\frac{\partial c^{*}}{\partial x^{*}}=0$ & at & $t^{*}>0$ & and & $x^{*}=0$ & and & $x^{*}=1$ \\
\hline$\frac{\partial c^{*}}{\partial y^{*}}=0$ & at & $t^{*}>0$ & and & $y^{*}=0$ & and & $y^{*}=$ \\
\hline
\end{tabular}

The zero-mass flux boundary condition, which implies that no mass is exchanged with the surroundings and is obtained from the derivation of the $\mathrm{CH}$ equation, is expressed in dimensionless form as:

$$
\begin{aligned}
& \left(\frac{\partial^{3} c^{*}}{\partial x^{* 3}}+\frac{\partial^{3} c^{*}}{\partial x^{*} \partial y^{* 2}}\right)=0 \quad \text { at } \quad t^{*}>0 \quad \text { and } \quad x^{*}=0 \quad \text { and } \quad x^{*}=1 \\
& \left(\frac{\partial^{3} c^{*}}{\partial x^{* 2} \partial y^{*}}+\frac{\partial^{3} c^{*}}{\partial y^{* 3}}\right)=0 \quad \text { at } \quad t^{*}>0 \text { and } y^{*}=0 \text { and } y^{*}=1
\end{aligned}
$$


In summary, the dependent variable is the dimensionless concentration $c^{*}$, and the independent variables are the dimensionless lengths, $x^{*}$ and $y^{*}$ and dimensionless time $t^{*}$ for two-dimensional model.

\subsection{Method of Solution}

Equation (4.12) with their related initial and boundary conditions are solved numerically using the Galerkin finite element method (GFEM) with a linear mesh of $31 \times 31$ elements. Hermitian basis functions are used to discretize space, and a first-order implicit Euler predictor-corrector method is used for time integration. The Newton-Raphson method is used to solve the set of equations resulting from the implementation of the Galerkin finite element method. An adaptive time-step controller is used to optimize the computational time. The values of the parameters used for the computation in this study are listed in chapter 5 . However, In this thesis, as already mentioned, double quench studies of phase separation have been investigated from a different pathway: quenching from one-phase region into the metastable region and a subsequent (shallow and deep) quench into the unstable region in order to verify the transition procedure and its cavity morphology resulting from the deep quench from nucleation and growth mechanism into spinodal decomposition mechanism. The Galerkin finite element method has been widely used for solving problems governed by ordinary differential equations, partial differential equations and integral equations in many applications. When we suppose that the given differential equation with appropriate initial condition and boundary conditions for two dimensional problems is expressed as: 
$L(u)=0 \quad$ for $\quad a \leq x \leq b \quad$ and $\quad a \leq y \leq b$

then an approximation solution $u_{a}$ can be assumed to be written as:

$u_{a}(x, y, t)=\sum_{j=1}^{N} u_{j}(t) \phi^{j}(x, y)$

where $\phi^{j}$ 's are known analytical functions, called global trial and test functions and $u_{j}$ 's are time dependent unknown coefficients. Replacing $u$ in equation (4.18) with $u_{a}$ in equation (4.19), we can obtain the following expression:

$$
L\left(u_{a}\right)=R \neq 0
$$

where $R$ is the residual. An approximation solution $u_{a}$ is assumed to be presented in a piecewise approximate function, so that if this approximation is correctly constructed. Then it will approach the corresponding exact solution $u$. In other words, the aim of this method of residual is to force $R$ to zero and consequently to make an approximate solution $u_{a}$ approach the exact solution. To do this, we need to set the inner product of the residual $R$ and an independent weight function $w$, equal to zero:

$F_{i}=\iint R w_{i}(x, y) d x d y=\iint L\left(u_{a}\right) w_{i}(x, y) d x d y=0$

In the Galerkin method, the weighted function $w$ is chosen from the same family of function in equation (4.19), therefore equation (4.21) can be written as: 
$F_{i}=\iint R \phi^{j}(x, y) d x d y=\iint L\left(u_{a}\right) \phi^{j}(x, y) d x d y=0$

where $i, j=1,2, \ldots . N$ and $F_{i}$ is the residual vector. It should be noted that equation (4.22) can be expressed as a set of $N$ differential equations to be solved for the unknown coefficients $u_{a}$ when we use the Gaussian integration. Consequently equation (4.18) has been reduced to a set of ordinary differential equations (4.22). The system of equations can be arranged in the form of:

$[J]\{u\}=[F]$

where $[J]$ is the Jacobian matrix expressed as:

$J_{i j}=\frac{\partial F_{i}}{\partial u_{j}}$

$u_{j}$ 's are the unknown coefficients of interest and $F_{i}$ is the residual vector. The NewtonRaphson iteration method is used to solve the set of equations obtained from equation (4.23). Considering equation (4.5) the residual vector can be written as:

$R=\frac{\partial c}{\partial t}-\nabla M \nabla\left(\frac{\partial f(c)}{\partial c}\right)-M \nabla^{2}\left(\frac{\partial f(c)}{\partial c}\right)+2 \kappa \nabla M \nabla^{3} c+2 \kappa M \nabla^{4} c$

Replacing (4.25) in equation (4.22) would result to:

$$
\begin{aligned}
& F_{i}=\iint R \phi^{i} d x d y=\iint \frac{\partial c}{\partial t} \phi^{i} d x d y-\iint \nabla M \nabla\left(\frac{\partial f(c)}{\partial c}\right) \phi^{i} d x d y-\iint M \nabla^{2}\left(\frac{\partial f(c)}{\partial c}\right) \phi^{i} d x d y \\
& +\iint 2 \kappa \nabla M \nabla^{3} c \phi^{i} d x d y+\iint 2 \kappa M \nabla^{4} c \phi^{i} d x d y
\end{aligned}
$$


To lower the order of the derivatives of equation (4.26), divergence theorem can be used:

$$
\int_{v} \alpha \nabla \cdot \hat{\beta} d v=\int_{s} \alpha \hat{n} \cdot \hat{\beta} d s-\iint_{v} \nabla \alpha \cdot \hat{\beta} d v
$$

where $\alpha$ can be any scalar and $\beta$ can be any vector. By applying divergence theorem several times and after applying boundary conditions the resulting equation in terms of the dimensionless residual vector $F_{i}$ would be:

$$
\begin{aligned}
& F_{i}=\int_{0}^{1} \int_{0}^{1} \frac{\partial c^{*}}{\partial t^{*}} \phi^{i} d x^{*} d y^{*}-\int_{0}^{1} \int_{0}^{1} D^{*} T^{*}\left[-\frac{1}{N_{1}}+\frac{1}{N_{2}}-2 \chi\left(1-2 c^{*}\right)\right]\left[\frac{\partial c^{*}}{\partial x^{*}} \frac{\partial c^{*}}{\partial x^{*}}+\frac{\partial c^{*}}{\partial y^{*}} \frac{\partial c^{*}}{\partial y^{*}}\right] \phi^{i} d x^{*} d y^{*}- \\
& \int_{0}^{1} \int_{0}^{1} D^{*} T^{*}\left[\frac{1-c^{*}}{N_{1}}+\frac{c^{*}}{N_{2}}-2 \chi c^{*}\left(1-c^{*}\right)\right]\left(\frac{\partial^{2} c^{*}}{\partial x^{* 2}}+\frac{\partial^{2} c^{*}}{\partial y^{* 2}}\right) \phi^{i} d x^{*} d y^{*}+ \\
& \int_{0}^{1} \int_{0}^{1}\left(1-2 c^{*}\right)\left[\frac{\partial c^{*}}{\partial x^{*}} \frac{\partial \phi^{i}}{\partial x^{*}}+\frac{\partial c^{*}}{\partial y^{*}} \frac{\partial \phi^{i}}{\partial y^{*}}\right]\left(\frac{\partial^{2} c^{*}}{\partial x^{* 2}}+\frac{\partial^{2} c^{*}}{\partial y^{* 2}}\right) d x^{*} d y^{*}+ \\
& \int_{0}^{1} \int_{0}^{1} c^{*}\left(1-c^{*}\right)\left(\frac{\partial^{2} c^{*}}{\partial x^{* 2}}+\frac{\partial^{2} c^{*}}{\partial y^{* 2}}\right)\left(\frac{\partial^{2} \phi^{i}}{\partial x^{* 2}}+\frac{\partial^{2} \phi^{i}}{\partial y^{* 2}}\right) d x^{*} d y^{*}
\end{aligned}
$$

equation (4.28) is then placed into the equation (4.24) in order to form the Jacobian matrix:

$$
\begin{aligned}
& J_{i, j}=\int_{0}^{1} \int_{0}^{1} \frac{\phi^{i} \phi^{j}}{\Delta t} d x^{*} d y^{*}-2 D^{*} T^{*} \phi^{i} \int_{0}^{1} \int_{0}^{1}\left\{2 \chi \phi^{i}\left[\frac{\partial c^{*}}{\partial x^{*}} \frac{\partial c^{*}}{\partial x^{*}}+\frac{\partial c^{*}}{\partial y^{*}} \frac{\partial c^{*}}{\partial y^{*}}\right]+\right. \\
& \left.\left[-\frac{1}{N_{1}}+\frac{1}{N_{2}}-2 \chi\left(1-2 c^{*}\right)\right]\left[\frac{\partial c^{*}}{\partial x^{*}} \frac{\partial c^{*}}{\partial x^{*}}+\frac{\partial c^{*}}{\partial y^{*}} \frac{\partial c^{*}}{\partial y^{*}}\right]\right\} d x^{*} d y^{*}- \\
& D^{*} T^{*} \phi^{i} \int_{0}^{1} \int_{0}^{1}\left\{\left[-\frac{1}{N_{1}}+\frac{1}{N_{2}}-2 \chi\left(1-2 c^{*}\right)\right]\left(\frac{\partial^{2} c^{*}}{\partial x^{* 2}}+\frac{\partial^{2} c^{*}}{\partial y^{* 2}}\right) \phi^{j}+\right. \\
& \left.\left[\frac{1-c^{*}}{N_{1}}+\frac{c^{*}}{N_{2}}-2 \chi c^{*}\left(1-c^{*}\right)\right]\left(\frac{\partial^{2} \phi^{j}}{\partial x^{* 2}}+\frac{\partial^{2} \phi^{j}}{\partial y^{* 2}}\right)\right\} d x^{*} d y^{*}+
\end{aligned}
$$




$$
\begin{aligned}
& \int_{0}^{1} \int_{0}^{1}\left\{-2 \phi^{j}\left[\frac{\partial c^{*}}{\partial x^{*}} \frac{\partial \phi^{i}}{\partial x^{*}}+\frac{\partial c^{*}}{\partial y^{*}} \frac{\partial \phi^{i}}{\partial y^{*}}\right]\left(\frac{\partial^{2} c^{*}}{\partial x^{* 2}}+\frac{\partial^{2} c^{*}}{\partial y^{* 2}}\right)+\right. \\
& \left.\left(1-2 c^{*}\right)\left[\left(\frac{\partial \phi^{i}}{\partial x^{*}} \frac{\partial \phi^{j}}{\partial x^{*}}+\frac{\partial \phi^{i}}{\partial y^{*}} \frac{\partial \phi^{j}}{\partial y^{*}}\right)\right]\left(\frac{\partial^{2} c^{*}}{\partial x^{* 2}}+\frac{\partial^{2} c^{*}}{\partial y^{* 2}}\right)+\left(\frac{\partial c^{*}}{\partial x^{*}} \frac{\partial \phi^{i}}{\partial x^{*}}+\frac{\partial c^{*}}{\partial y^{*}} \frac{\partial \phi^{i}}{\partial y^{*}}\right)\left(\frac{\partial^{2} \phi^{i}}{\partial x^{* 2}}+\frac{\partial^{2} \phi^{j}}{\partial y^{* 2}}\right)\right\} d x^{*} d y^{*}+ \\
& \int_{0}^{1} \int_{0}^{1}\left\{\phi^{j}\left(1-2 c^{*}\right)\left(\frac{\partial^{2} c^{*}}{\partial x^{* 2}}+\frac{\partial^{2} c^{*}}{\partial y^{* 2}}\right)+c^{*}\left(1-c^{*}\right)\left(\frac{\partial^{2} \phi^{i}}{\partial x^{* 2}}+\frac{\partial^{2} \phi^{i}}{\partial y^{* 2}}\right)\right]\left(\frac{\partial^{2} \phi^{i}}{\partial x^{* 2}}+\frac{\partial^{2} \phi^{i}}{\partial y^{* 2}}\right) d x^{*} d y^{*}
\end{aligned}
$$

Hermitian bicubic basis functions are then used to solve the resulting fourth-order partial differential equation (4.29). This type of basis function is applied due to its capability of minimizing the order lowering of the partial differential equations and being a good basis function in discretizing space. The first-order implicit Euler predictor-corrector method is used for time integration. The Newton-Raphson method is used to solve the set of equations resulting from the implementation of the Galerkin finite element method. An adaptive timestep controller is also used to optimize the computational time. The resulting set of dimensionless governing equations was adapted to the FORTRAN 77 source codes written by Chan [30-34] for solving the phase separation in polymer solutions with constant mobility. 


\section{Chapter 5}

\section{Results and Discussion}

Paying attention to Figure 1.8 reveals that the phase diagrams are symmetric in both cases.

Generally, working with symmetric or nearly symmetric phase diagrams are much easier task for numerical simulation purposes. That is why most researchers prefer to model these types of mixtures. What actually makes this research different from the other related studies is that the simulated model is completely asymmetric which is better in representing the real polymer solutions. Figure 5.1 (a) is depicting an asymmetric phase diagram of a polymer solution. In this case, a critical quench is performed from one-phase region to unstable region (to $\mathrm{G}$ ) and a subsequent quench either to metastable region (to $\mathrm{H}$ ) representing a shallow quench or to unstable region (to I) featuring a deep quench. Considering a homogeneous binary polymer solution; where the components are a polymer and a solvent and according to the numerical and experimental work performed by several groups explained in the literature review, the expected morphology for the two-step deep quench would be polymer droplets (secondary droplets) appearing inside the solvent interconnected (due to critical quench) matrix. 


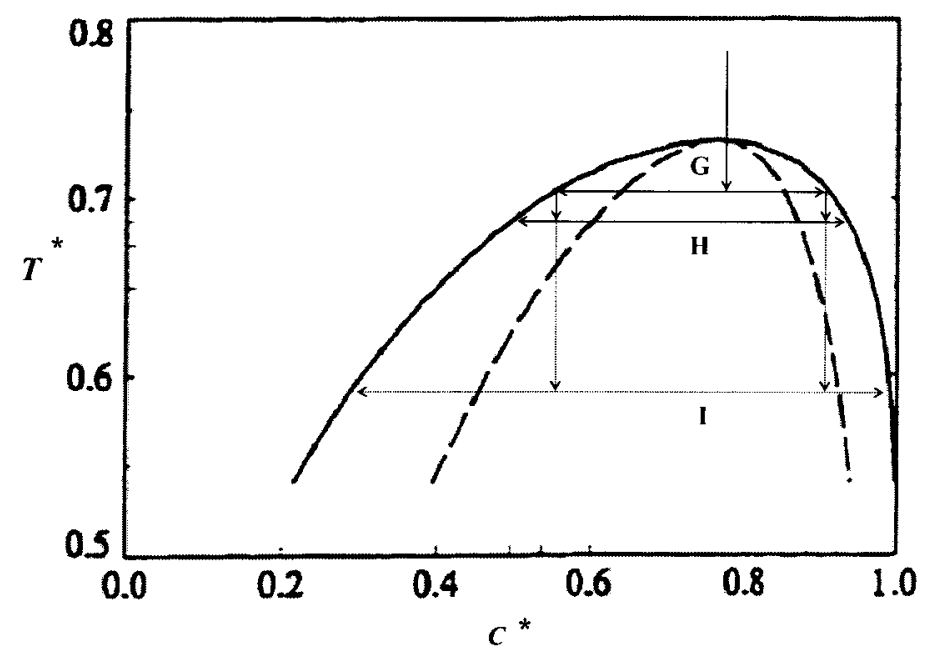

(a)

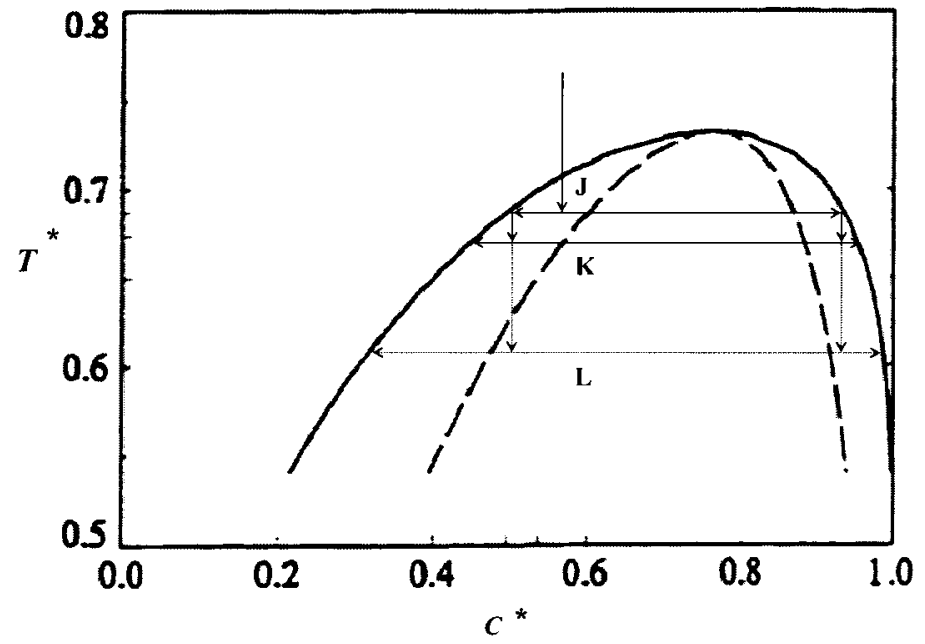

(b)

Figure 5.1: Schematic phase diagram of TIPS process. The solid (dashed) curves represent the binodal (spinodal) lines. In case (a); the solution undergoes a critical quench into G first and then to $\mathrm{H}$ or I for shallow or deep quench respectively, while in case (b); the solution undertakes a critical quench into $\mathrm{J}$ first and then to $\mathrm{K}$ and $\mathrm{L}$ for shallow and deep quenches respectively. 
This type of morphology as already stated was also verified for a polymer blend with a symmetric phase diagram in one dimension by Chan et al [25]. Figure 5.2 (a) and (b) show, respectively, the evolution of the dimensionless spatial concentration profile for the critical quench after a shallow and deep second temperature jump in the two-step phase separation phenomenon.
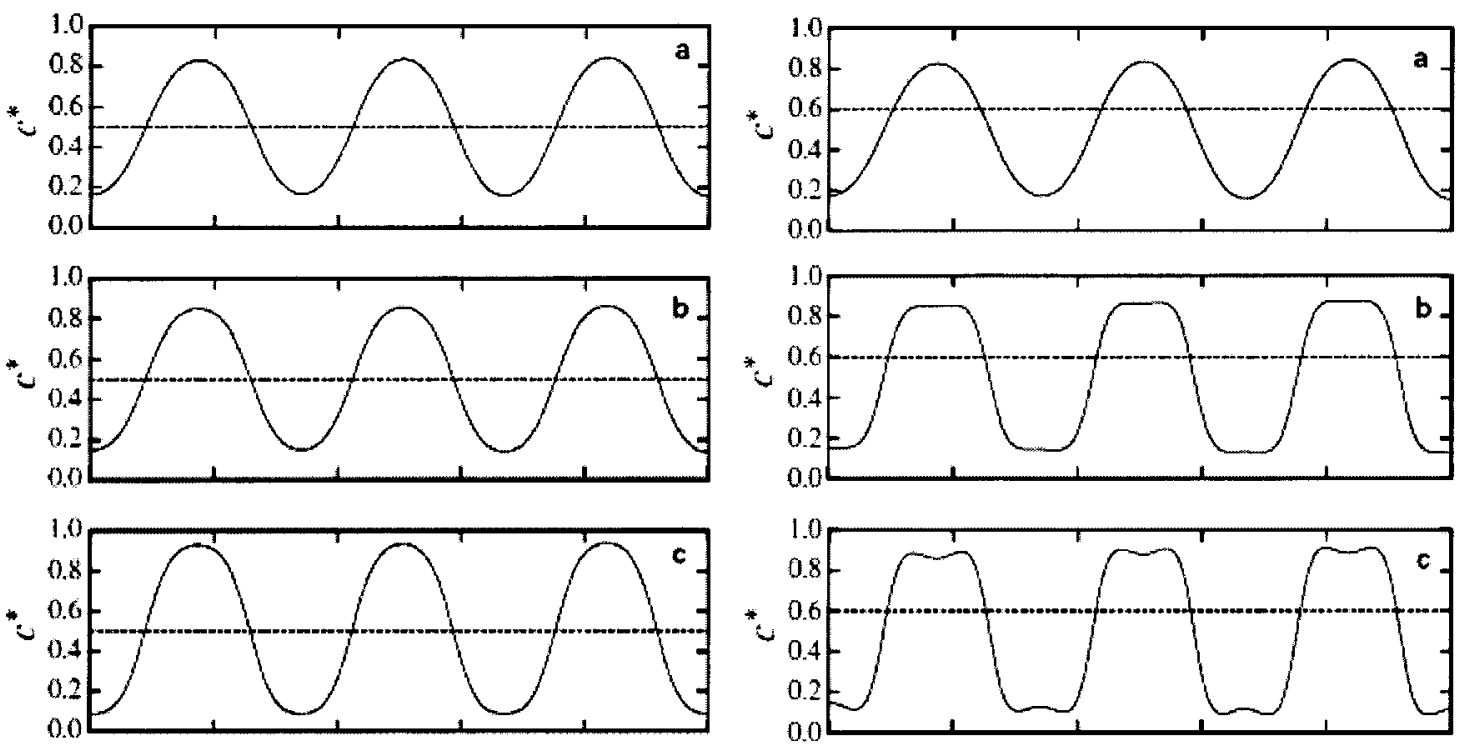

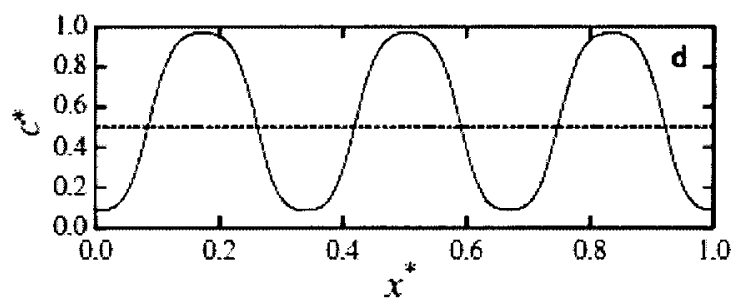

(a)

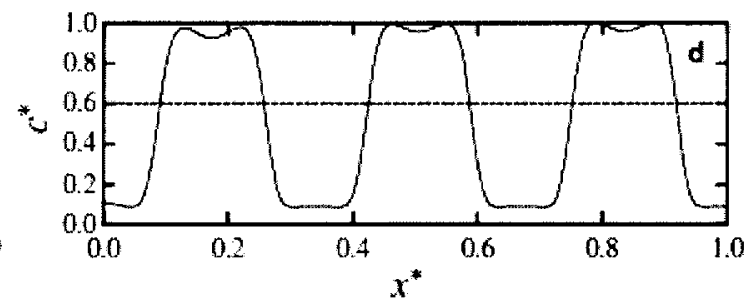

(b)

Figure 5.2: The evolution of the dimensionless spatial concentration profile for the (a) shallow and (b) deep two-step phase separation. The initial average concentration is $c_{0}^{*}=0.5$, and the dimensionless diffusion coefficient is $D^{*}=2 \times 10^{5}[25]$. 
Figure 5.2 (a) indicates that there is no evidence of double phase separation for a shallow quench case after the second temperature jump. What is observed in the evolution of the spatial concentration profiles is a continuous growth of the already growing initial profiles present in the initial quench. In the two-step deep quench case (Figure 5.2, b), there appears to be a secondary small peak forming in the evolution of the dimensionless spatial concentration profile. This small broad peak is not reflected in the evolution of the spatial concentration profile in Figure 5.2 (a) where a continuous increase of the original growth of the concentration fluctuations is observed.

Figure 5.1 (b) shows the polymer solution undertaking an off critical quench into $J$ first and then to $\mathrm{K}$ and $\mathrm{L}$ for shallow and deep quenches respectively. As mentioned in advance, there has been no numerical or experimental evidence of obtaining the secondary droplets by imposing a double quench on a polymer solution with an asymmetric phase diagram. That is why the previous studies on double quench phase separation have considered a symmetric mixture for their modeling or morphological analyses.

\subsection{Off-Critical Double Quench within NG}

It is now the aim of this work to study a new pattern formation caused by a second quench within the metastable region where the dominant phase separation mechanism is nucleation and growth. So far, only a few numerical investigations [49,78] have been reported and mostly experimental work have been done on this special type of phase separation. Even 
among the numerical and experimental work, our approach to the resulting morphology is different from the others. For the first time, the off-critical double quench within NG region has been verified using Cahn-Hilliard nonlinear equation to describe the dynamic behavior of phase separation via NG and SD and the Flory-Huggins free-energy expression to describe the thermodynamic behavior of the model polymer solution. The reason that we focused on a polymer-solvent solution is that in polymer-polymer mixtures, we see only the rather early stage of a second phase separation before the large-scale exchange of materials over the original domains, because of slow dynamics in polymer mixtures. The overall pattern evolution caused by a double quench can be observed much easier in polymer-solvent mixtures than in polymer-polymer mixtures since the elementary diffusion process is much faster in the former than in the latter.

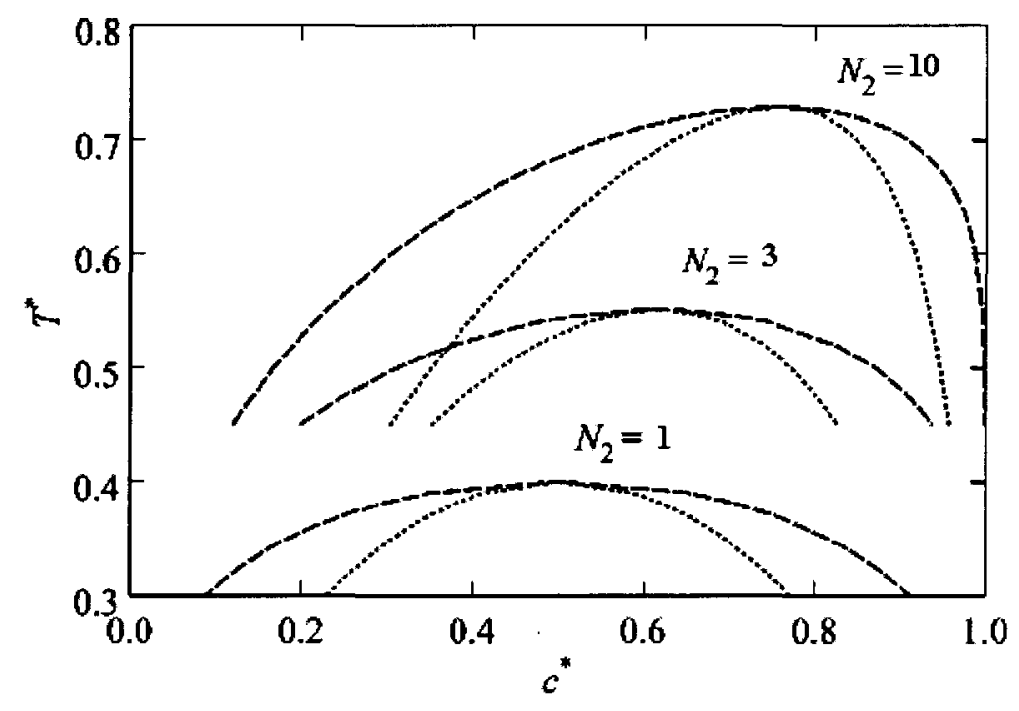

Figure 5.3: Schematic representation of a PDLC phase diagram curvature from symmetric state into asymmetric one [13]. 
The model is then chosen to be a polymer solution composed of a polymer and a solvent. The phase diagram has been intentionally selected to be asymmetric in order to better feature the real polymer solutions. Figure 5.3 depicts the pathway of a polymer solution from symmetric state $\left(N_{2}=1\right)$ to asymmetric one $\left(N_{2}=10\right)$. As discussed in chapter 1, by increasing the degree of polymerization, the phase diagram tends to shift gradually to the right side. Next we describe the pattern evolution caused by a double quench composed of a first quench within the nucleation and growth region and a subsequent shallower quench within the same (metastable) region (Figure 5.4).

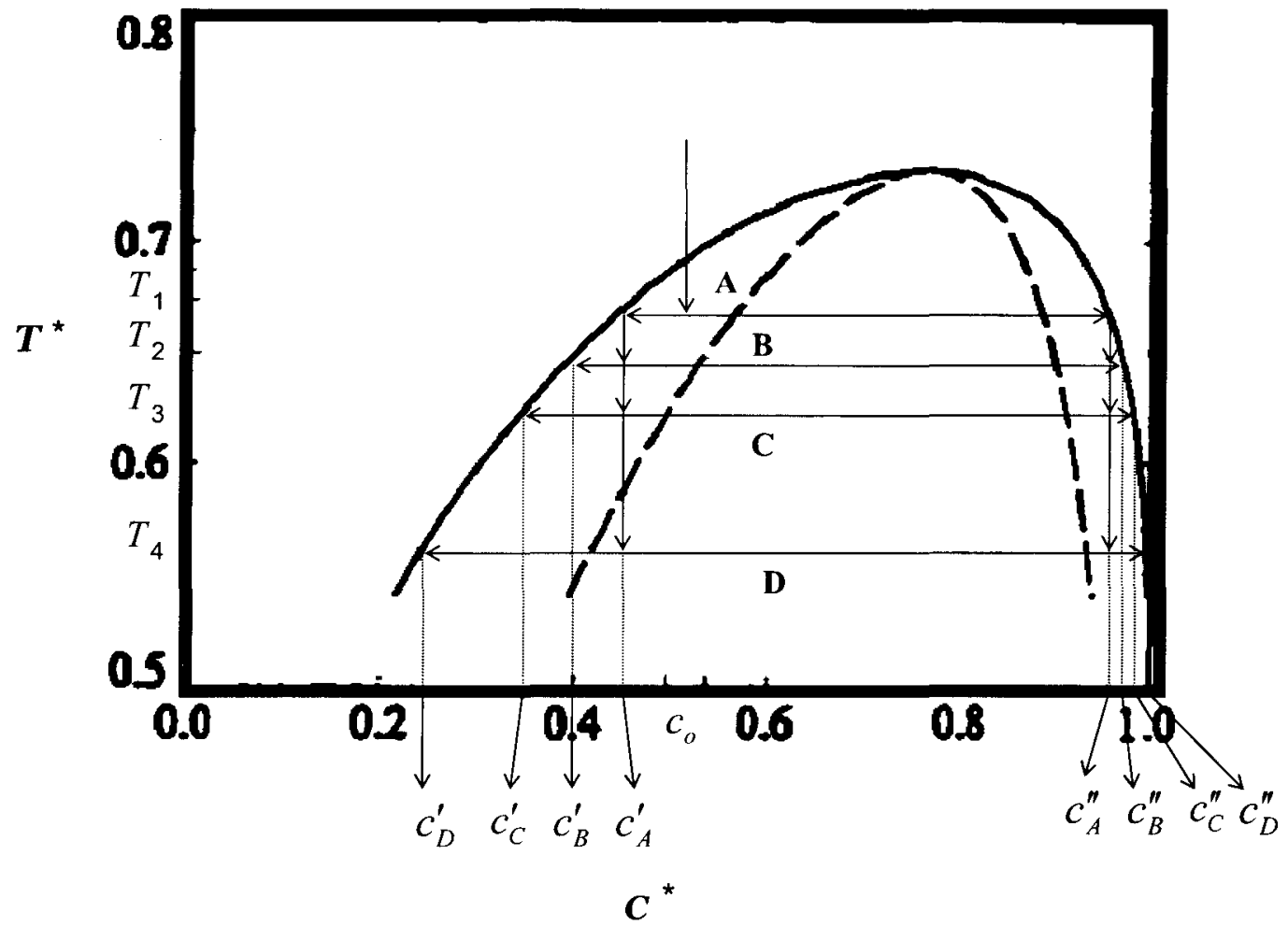

Figure 5.4: Schematic representation of TIPS in a two-step off-critical quench in an asymmetric polymer solution. The solid (dashed) line represents the binodal (spinodal) curve. 

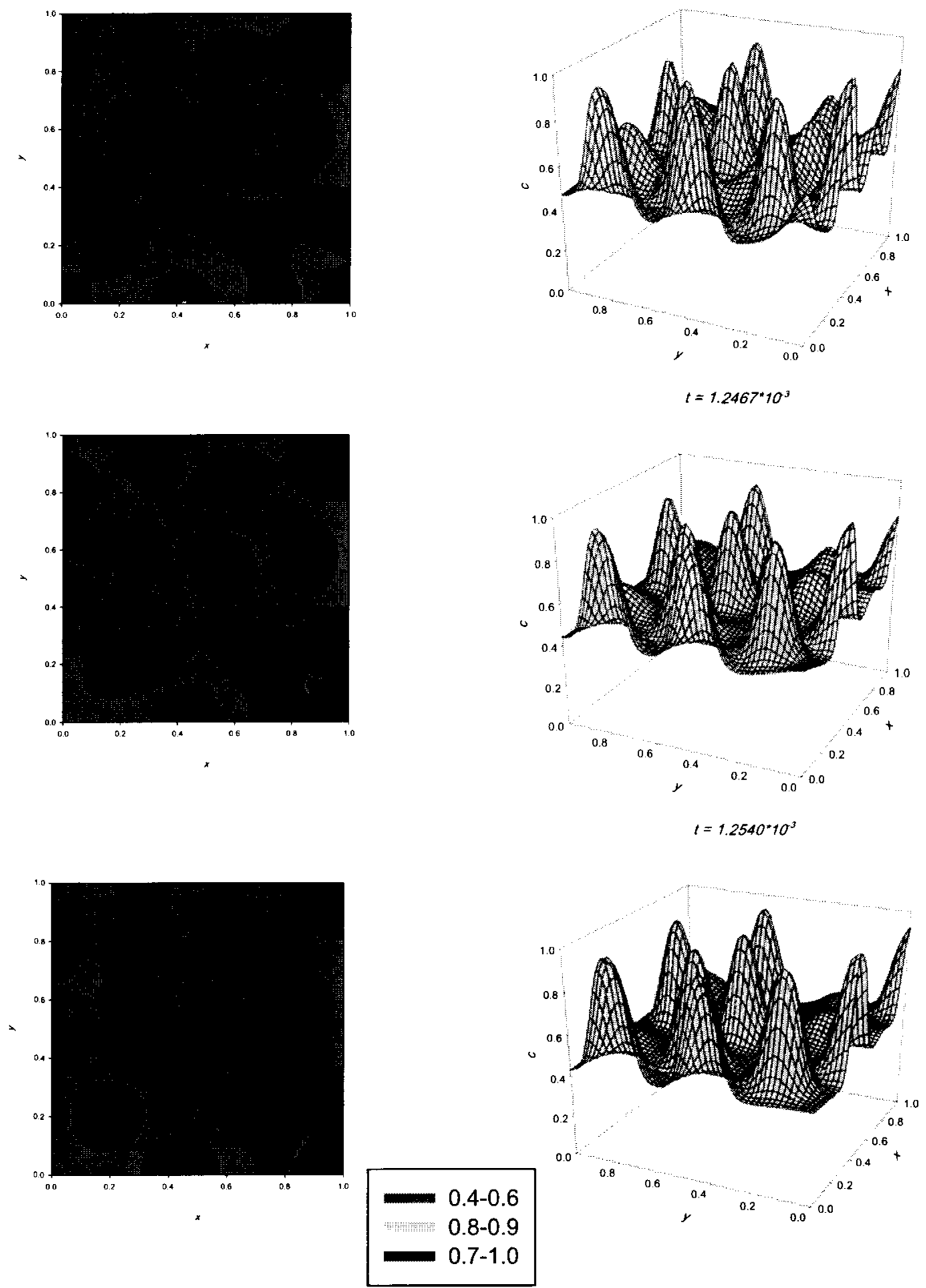

Figure 5.5: Dimensionless 2D contour spatial concentration profiles for $c^{*}\left(x^{*}, y^{*}\right)$ (left column) and 3D concentration fluctuation patterns (right column) for an off-critical shallow double quench case (A to $\mathrm{B}$ ) at the following dimensionless times respectively: $t^{*}=1.2386 \times 10^{-3}, t^{*}=1.2467 \times 10^{-3}$ and $t^{*}=1.2540 \times 10^{-3}$ where $D^{*}=5000$. 
The solution is first quenched from an arbitrary temperature above the binodal curve in which the solution is in its homogeneous state into the metastable region (A) where the temperature is $T_{1}$ (Table 5.1). After the commencement of phase separation, when solvent droplets and polymer phases are trying to reach the equilibrium concentrations $\left(c_{A}^{\prime}\right.$ and $\left.c_{A}^{\prime \prime}\right)$, at some elapsed time $\left(t^{*}=1.2337 \times 10^{-3}\right)$ given to the solution (the second quench was performed through numerous different transition times, see appendix A) the solution is quenched again to the new temperature $T_{2}$ (B) representing a shallow quench.

Table 5.1: Parameter values used for the shallow and deep double quench phase separation within NG and SD regions in TIPS method.

\begin{tabular}{ll}
\hline Parameter & Value \\
\hline$N_{1}$ & \\
$N_{2}$ & 1 \\
$\psi$ & 10 \\
$c_{c}{ }^{*}$ & 1 \\
$T_{c}{ }^{*}$ & 0.7597 \\
$D^{*}$ & 0.7319 \\
$c_{0}^{*}$ & $5 \times 10^{3}$ \\
$T_{1}^{*}$ & 0.545 \\
$T_{2}^{*}$ & 0.675 \\
$T_{3}^{*}$ & 0.65 \\
$T_{4}^{*}$ & 0.625 \\
$\chi$ & 0.56 \\
\hline
\end{tabular}


After this second quench, no secondary structure has been observed (Figure 5.5). The parameter values used in the simulation of Figure 5.5 case is listed in Table 5.1. The legend has been rescaled in order to simplify the contour and it will be the same for all the figures. Using different parameters (appendix A), the obtained morphology would be still the same. The solution then approaches the equilibrium concentrations $\left(c_{B}^{\prime}\right.$ and $\left.c_{B}^{\prime \prime}\right)$. This type of pattern formation, which looks the same as regular first step quench; is due to the fact that after the second quench both polymer and solvent will phase separate almost independently. Since the concentration curvature is not big enough (shallow quench) the driving force for making a new structure will be weak which can not affect the morphology (i.e. droplets) formed from the first quench. The morphology has already been investigated experimentally and reported by Tanaka [86]. Figure 5.5 shows the pattern formation of a shallow double quench in two and three dimensions. The contours have been enhanced with color for better observation and in order to be able to track the morphology changes. In this work, however, we are also mainly interested in the effects of the second deeper quench on the pattern evolution. In an asymmetric phase diagram or under an off-critical quench condition, the two coexisting phases could be brought into different states of instability by a further deeper quench. In case of second deeper quench, two sections were investigated: (i) second deep quench within NG region (A to $\mathrm{C}$ ) where both polymer and solvent phases are undergoing nucleation and growth mechanism and (ii) second deep quench (A to D) where polymer undergoes SD phase separation and solvent droplets will still follow NG mechanism namely, one phase becomes untastable, while the other metastable. In both categories, similarly the solution is first quenched from one phase region into the metastable region (A) where the temperature is $T_{1}$ (Table 5.1). 
After the first quench the polymer solution exhibits the usual droplet morphology based on the formation mechanism of nucleation and growth in the off-critical phase separation. As solvent droplets and polymer phase separate to reach the equilibrium concentrations $\left(c_{A}^{\prime}\right.$ and $\left.c_{A}^{\prime \prime}\right)$, the second quench is imposed on the system at different transition times (appendix A) down into the temperature $T_{3}=0.625$. When a further second quench is applied to the solution within NG region, the concentration in the centers of the solvent droplets instantaneously decreases. At the same time, the concentration at the edges of the droplets correspondingly increases, leading to the formation of a cavity structure [78]. After the second quench, however, a concentration adjustment is required at the boundary to establish local equilibrium. The diffusion flow caused by the concentration imbalance (due to the second quench) across the interface of polymer and solvent carries the polymer molecules towards the center of each solvent droplet [86]. During this process, the flow coming from the surroundings of the droplet starts to accumulate at the center of the solvent droplet (see Figures 5.7 and 5.9). This again causes the droplet to have excess polymer components, and accordingly a new non-equilibrium state [181]. Then the redistribution of concentration further proceeds to establish a final equilibrium. Figure 5.6 (a) shows at $t^{*}=1.2240 \times 10^{-3}$ a light concentration polymer drop occurs in the middle of solvent droplet. With the increasing time, these typical secondary domain structures disappear gradually. The original morphology is returned but with higher concentration contrast between two phases. Then, the coalescence of the cavities occurs (Figure $5.6 \mathrm{~b}$ ), and the irregularly anisotropic droplets gradually shrink and reshape into circular ones. With the increasing time, some small cavities shrink and disappear, which leads to the decrease of the total cavity number. 

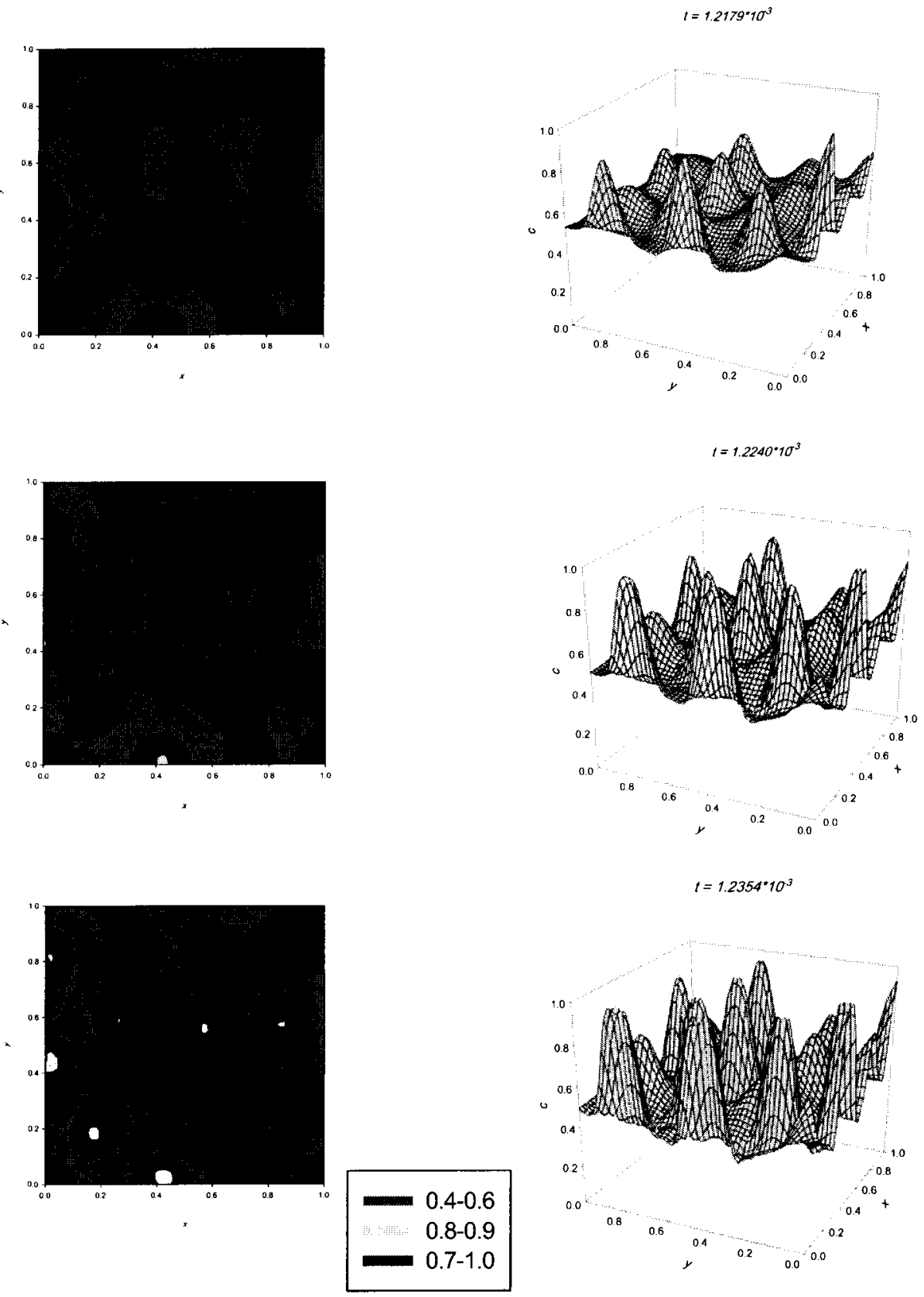

Figure 5.6(a): Dimensionless 2D contour spatial concentration profiles for $c^{*}\left(x^{*}, y^{*}\right)$ (left column) and 3D concentration fluctuation patterns (right column) for an off-critical deep double quench case within $\mathrm{NG}$ region (A to $\mathrm{C}$ ) at the following dimensionless times respectively: $t^{*}=1.2179 \times 10^{-3}, t^{*}=1.2240 \times 10^{-3}$ and $t^{*}=1.2354 \times 10^{-3}$ where $D^{*}=5000$. 

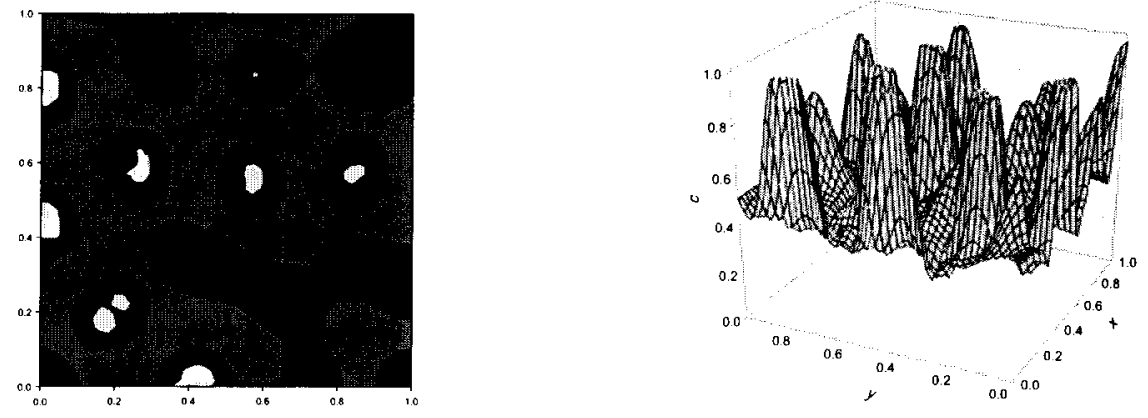

$t=1.2563^{*} 10^{3}$
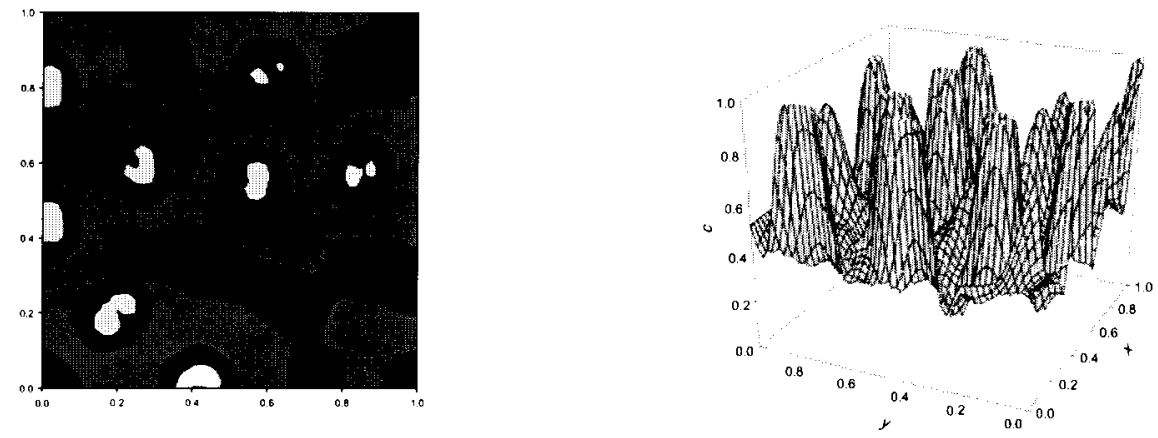

$t=1.2686^{*} 1 \sigma^{3}$
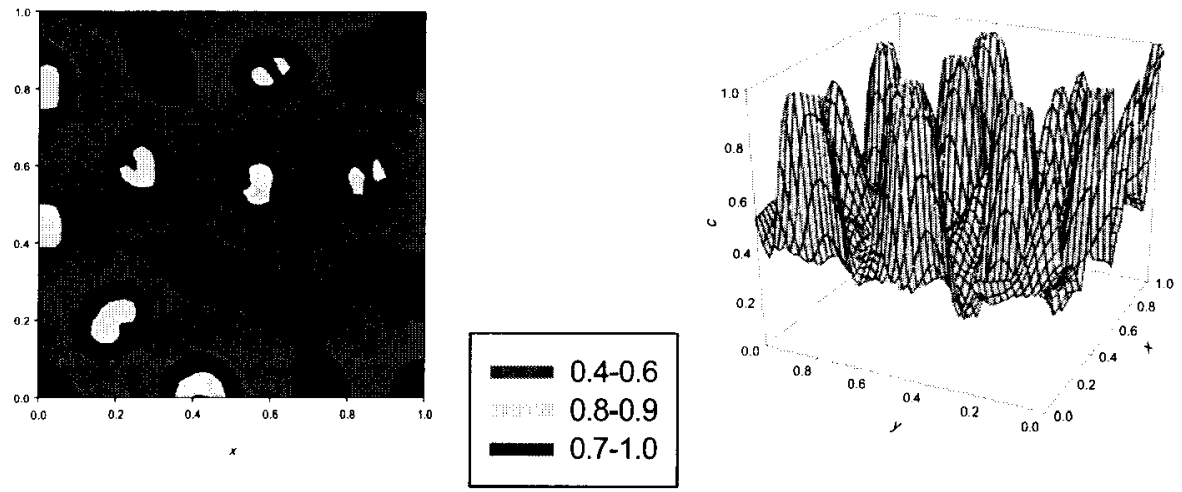

Figure 5.6(b): Dimensionless 2D contour spatial concentration profiles for $c^{*}\left(x^{*}, y^{*}\right)$ (left column) and 3D concentration fluctuation patterns (right column) for an off-critical deep double quench case within NG region (A to $\mathrm{C}$ ) at the following dimensionless times respectively: $t^{*}=1.2459 \times 10^{-3}, t^{*}=1.2563 \times 10^{-3}$ and $t^{*}=1.2686 \times 10^{-3}$ where $D^{*}=5000$. 
Figures $5.6 \mathrm{a}$ and $\mathrm{b}$ show the three-dimensional (3D) diagrams of the concentration fluctuation with their corresponding contour graphs highlighting a more detailed development of the secondary domain structures. In the $3 \mathrm{D}$ diagrams, the cavity of the solvent droplets fall down when a further second quench is applied to the solution, demonstrating the formation of the secondary domain structures. These falling parts will rise again and reach to a new altitude with higher concentration (Figures 5.7 and 5.10). The structure developed before the second-step T-jump can be regarded as the initial structure for the second-step phase separation process. When the temperature is brought to $T_{3}$ the driving force of the phase separation would increase.

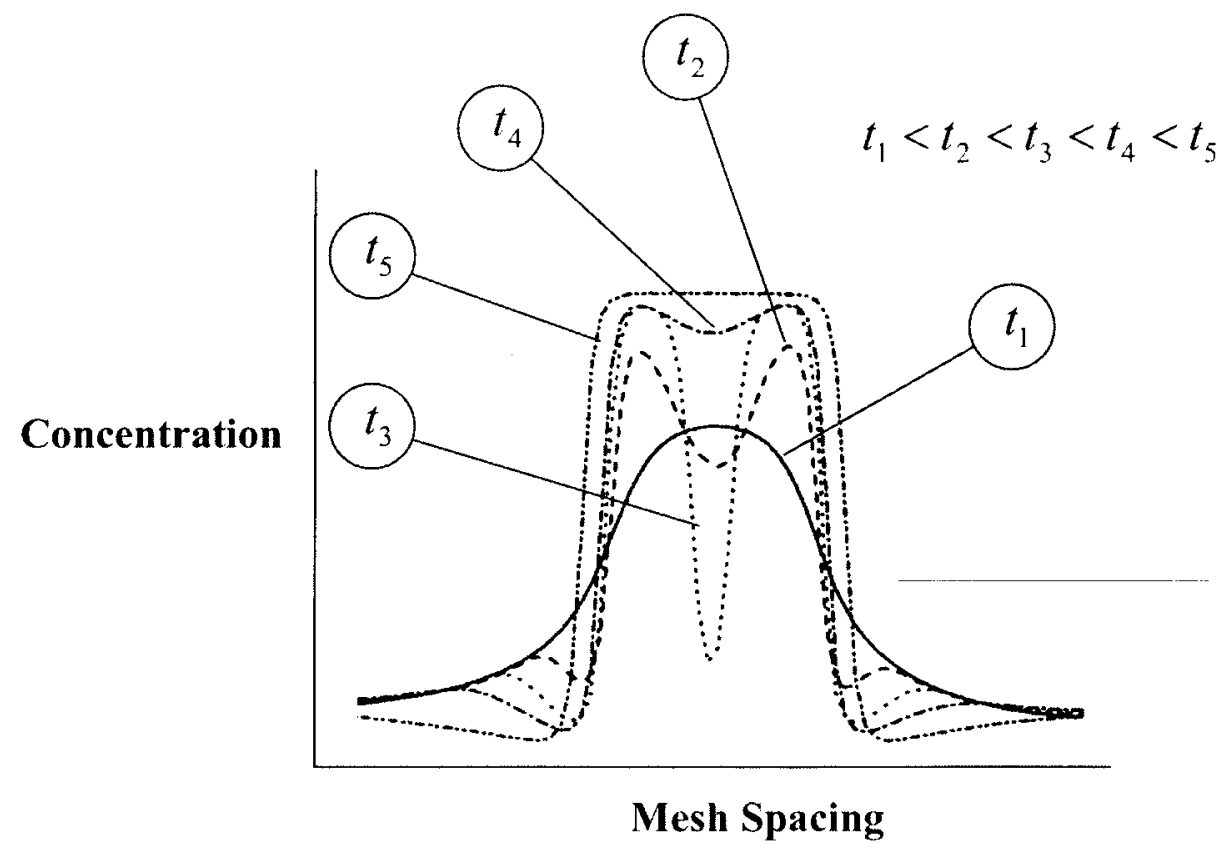

Figure 5.7: Typical time evolution of the one-dimensional cross-section through one of the droplets depicting the formation and dissolution of a cavity. 
On the other hand, the two pattern evolutions of polymer and solvent with different spatial scales are strongly coupled with each other through the global diffusion between the solvent droplets and the polymer matrix. During the late stage, the composition difference between solvent droplets and the polymer causes the diffusion flow from solvent droplets into the polymer matrix which leads to cavity disappearance. Thus the cavities gradually disappear from the neighborhood of the solvent droplets (see Figures 5.7 and 5.10). Another pathway of double quench phase separation is when we lower the temperature down enough to $T_{4}$ inside the unstable region (A to D). Here, the polymer matrix enters the unstable region (Figure 5.4), therefore the governing phase separation mechanism for polymer would be spinodal decomposition while for the solvent droplets; phase separation is still followed by NG. Since solvent droplets are almost reaching their equilibrium state, polymer mixture is playing an important role in the morphology. As it is clear from Figure 5.8, the cavity patterns are almost the same as the earlier case. After the crossover from NG to SD there is always the possibility that the diffusion process cannot catch up with the geometrical growth of domains and the domains may become out of equilibrium. Once the diffusion of solvent molecules cannot follow the geometrical growth, a secondary phase separation occurs inside the droplets, in which the size of the droplet grows as usual. It should be noted that although experimentally the mobility is temperature and hence quench depth dependent, it is not necessary to explicitly account for this since the difference between mobility during the first and second quench can be reflected in a different scaling factor from numerical time to real time for each of the two stages. Importantly, the structural growth is not affected by this assumption. 

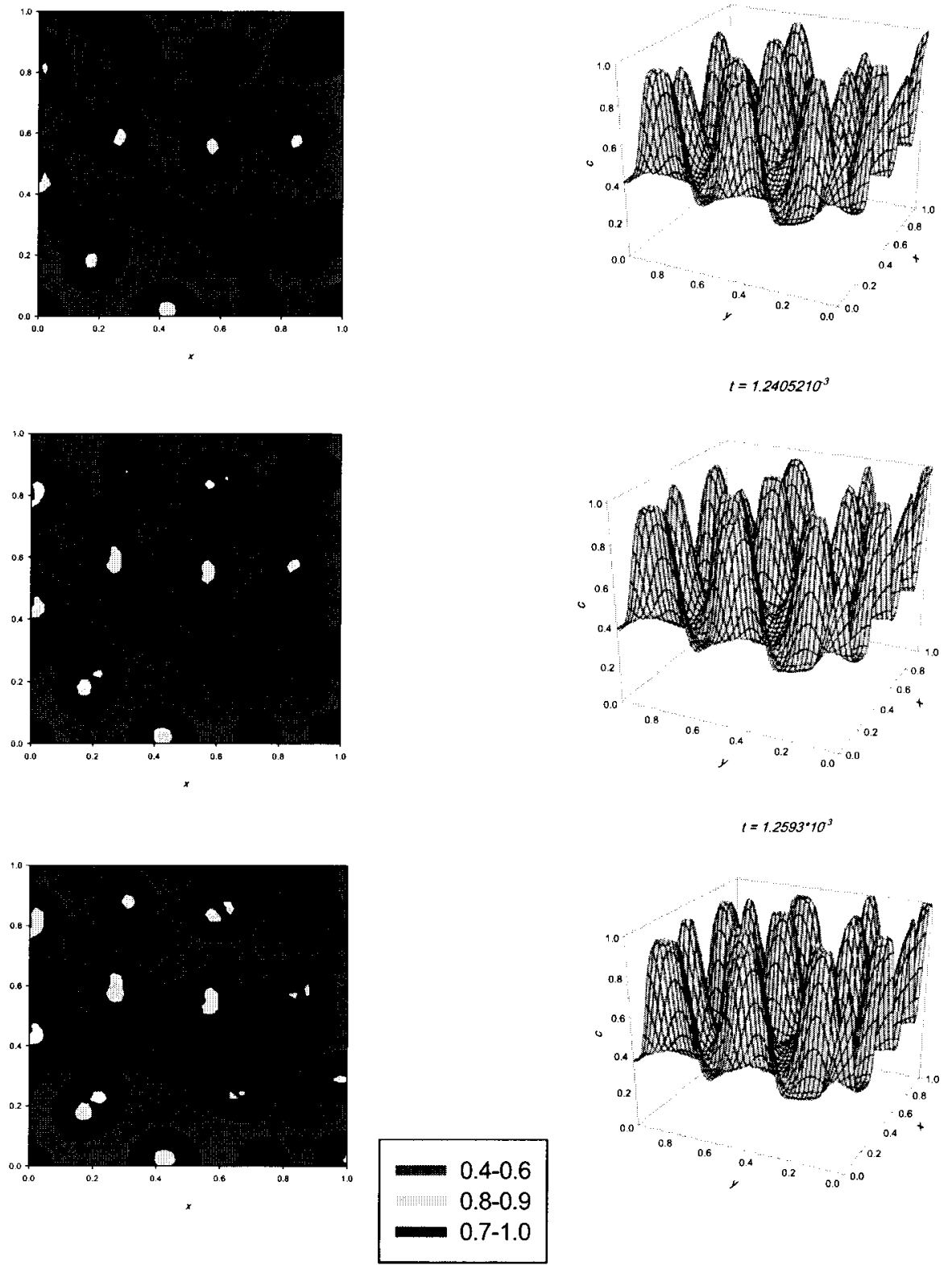

Figure 5.8: Dimensionless 2D contour spatial concentration profiles for $c^{*}\left(x^{*}, y^{*}\right)$ (left column) and 3D concentration fluctuation patterns (right column) for an off-critical deep double quench case within SD region for polymer phase and NG region for solvent phase (A to $\mathrm{D})$ at the following dimensionless times respectively: $t^{*}=1.2349 \times 10^{-3}, t^{*}=1.2405 \times 10^{-3}$ and $t^{*}=1.2593 \times 10^{-3}$ where $D^{*}=5000$. 
As the simulation proceeds, small cavity domains appear in the solvent domains (Figures 5.8). Although these cavities are initially created, they dissolve into the surrounding solvent since they create large interfacial areas and a large increase in free energy. The second-order system starts to lose the cavity due to its flux toward the interface of the solvent and polymer. The smaller a cavity is, the higher the concentration of the solvent rich droplet phase at the matrix side boundary of the cavity is.

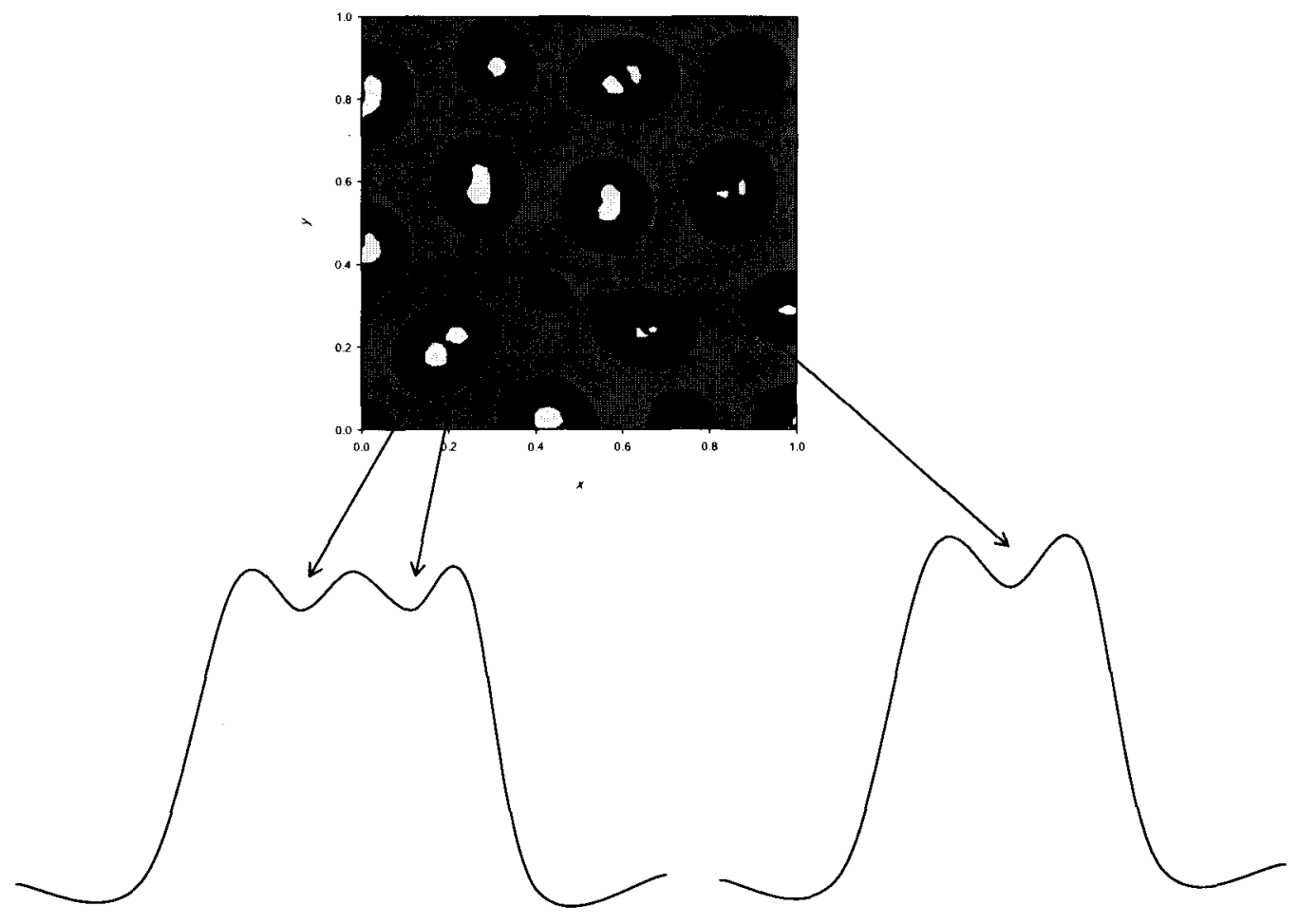

Figure 5.9: Schematic cross section of concentration profile corresponding to cavity formation in a droplet. 
The interface of the first-order droplet should be regarded as the boundary for small secondary cavities. So the concentration near the inner boundary of the first-order solventrich droplet must be lower than the final equilibrium value of the solvent-rich phase. Solvent droplets that are rich in the second order cavity will lose the cavity as polymer molecules are transferred via diffusion to the inside of the polymer matrix. Accordingly, the second-order cavities evaporate and eventually disappear completely (Figure 5.10).

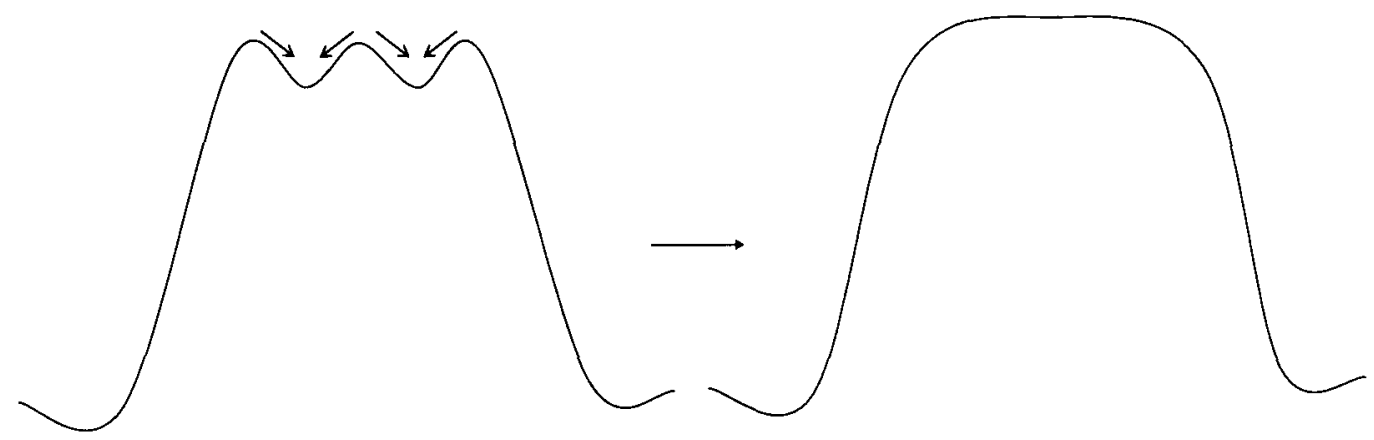

Figure 5.10: Schematic cross section of concentration profile representing the cavity deformation in a droplet.

However, it takes some time for this diffusion process to be really operative. On the other hand, since the contribution of the volume part to the free energy is much larger than that of the interface part, the volume ratio between the solvent droplet and the cavity first approaches its final one. This imbalance and the resulting diffusion field between the solvent droplet and the regions having polymer components cause the cavity deformation, namely a kind of interface instability. 
Up to this stage, the initial droplet distribution just before the second quench strongly affects the pattern formation. Finally the shape relaxation occurs very slowly to reduce the total interfacial energy. This process accompanies the complete reorganization of droplet shape and droplet distribution. However, as the driving force coming from the interface energy is too weak for a quick, large-scale reorganization, this final process is likely very slow. It should be noted that this kind of cavity occurs only for a second quench within the metastable region.

The simulated morphology for the primary phase separation structure shows solvent droplets dispersed in a matrix of polymer. Increasing solvent concentration increases the size of the droplets, but the growth behavior remains similar. During the second deeper quench, the equilibrium compositions are not constant; they move apart. Both polymer and phase separated solvent obtained from the first quench undergo another phase separation independently. Therefore the coexisting phases have to change their composition continuously by material exchange. Due to domain growth and the increasing distances, material exchange by diffusion becomes more and more difficult. At a certain time the diffusion becomes too slow to follow the structure coarsening. At this time secondary demixing starts inside the primary domains, which are created by the primary phase separation. Based on this idea there would be a competition between structure coarsening and interdiffusion in double quenches. In Figure 5.6 (b), the last 3D concentration fluctuation pattern for an off-critical deep double quench case within NG region (A to C) at time $t^{*}=1.2686 \times 10^{-3}$ has been rescaled (Figure 5.11) for dimensionless concentration from -1 to 3 on purpose in order to make it easier for cavity observation. 


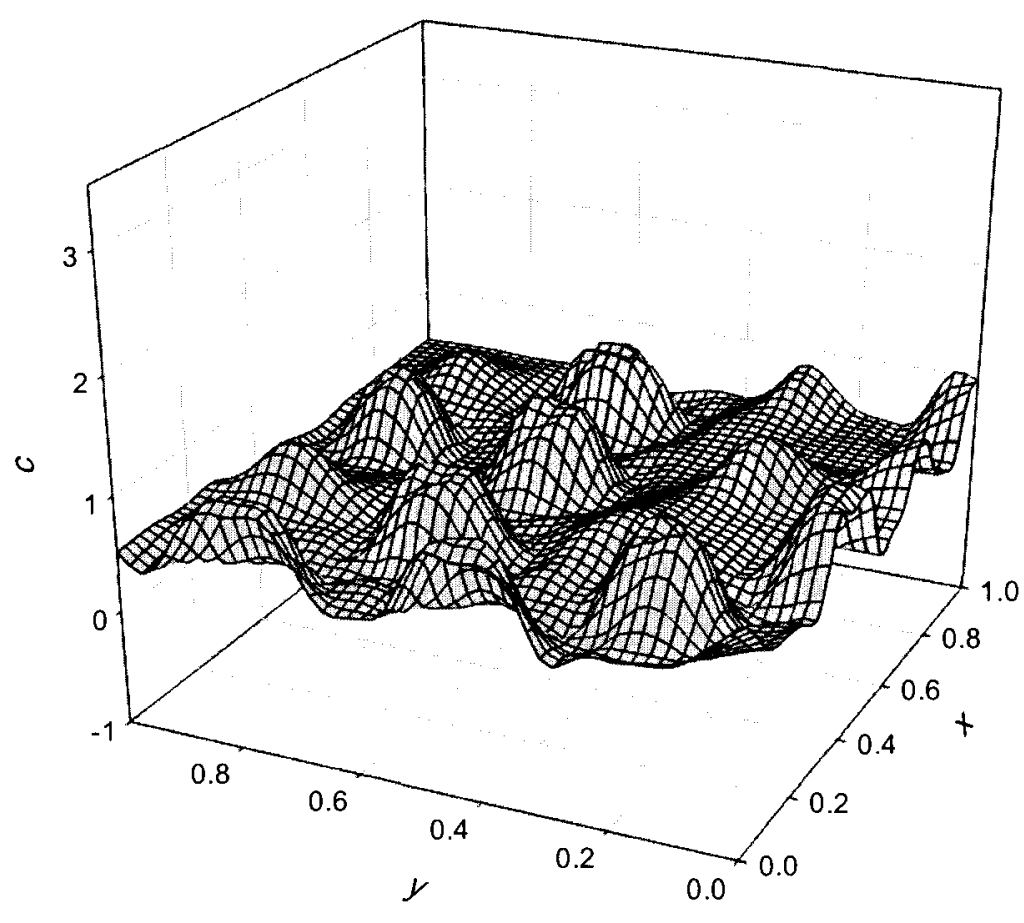

Figure 5.11: $3 \mathrm{D}$ concentration fluctuation patterns $c^{*}\left(x^{*}, y^{*}\right)$ for an off-critical deep double quench case within NG region (A to C) at $t^{*}=1.2686 \times 10^{-3}$.

When the scale is from 0 to 1 , one might have difficulty to see the cavities inside the droplets. The cavities are more conspicuous in Figure 5.11. Obviously, when the second quench is applied, the extreme concentration fluctuation occurs around each droplet. With the increasing time, the range of the fluctuation will expand and finally cover almost the whole phase region of the majority component. Hence, the height contrast between two phases is enlarged. Although the appearance of these secondary domain structures in both situations of off-critical conditions are brief, but they can still be used to tune the phase separation process 
by appropriate control methods and obtain the interesting phase structures that could be considered for the industrial applications such as electro-optical devices. One can also note that the secondary domain structures with deeper second quench depths are clearer than those with shallower ones demonstrating again that a deeper second quench depth can induce a more intensive concentration fluctuation and a smaller initially favored length scale.

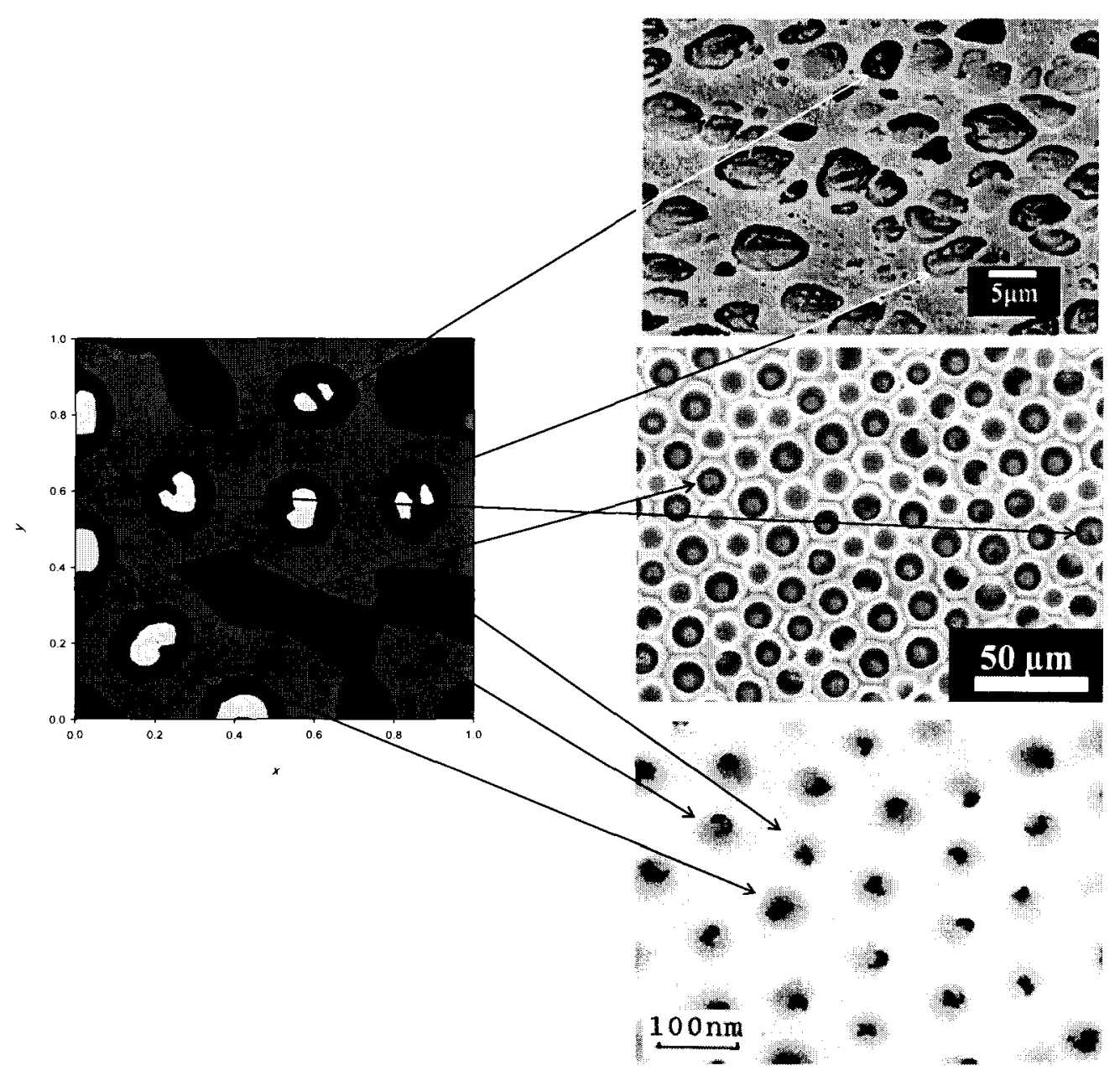

Figure 5.12: The comparison between the simulated morphology and the experimental results $[23,136,138]$. 
Clearly, the concentration in the middle of the droplet falls down at the initial time of the second quench process, corresponding to the formation of the light concentration polymer drop as shown in Figures 5.7 and 5.9. A clearer secondary domain structure may be obtained by tuning the initial phase structure of the second quench depth using different methods, e.g., the coarsening time. It is also sensible to compare the achieved morphology with the experimental photos. Figure 5.12 evaluates the simulated model with the experimental work of Ohshima et al. [64], Ishizu [136] and Meijer et al. [138] respectively. In all three, the cavities are inside the second component droplets whether as a bulk (core-shell) or droplets.

\subsection{Structure Factor Evolution in Shallow Double Quench}

The structure factor is an important parameter that is often used to characterize the shape of phase-separated domains by NG and SD and relates numerical and experimental studies. The numerical data of the computed composition fluctuations are used to calculate the structure factor by taking the fast Fourier transform. The relationship between experimental (the scattering intensity from light scattering experiments) and numerical work (the structure factor) is of the following form:

$$
I(q, t) \propto S^{*}\left(\mathbf{k}^{*}, t^{*}\right)=\left\|A^{*}\left(\mathbf{k}^{*}, t^{*}\right)\right\|^{2} \quad \text { for } \quad q=\mathbf{k}^{*}
$$

$I$ is the scattering intensity, $q$, is the scattering wave vector, $S$ is the structure factor and $\mathbf{k}^{*}$ is the wave number in Fourier space. The experimental light scattering data is then directly proportional to the structure factor [16]. 
From light scattering data, in the early stages of phase separation by SD, the scattering intensity grows exponentially and the scattering vector, $q$, is independent of time .The growth of the composition fluctuations is weakly nonlinear. Plotting the natural log of the scattering intensity evolution:

$$
I(q, t)=I(q, t=0) \exp (2 R(q) t)
$$

where $R(q)$ is a growth rate of the concentration fluctuation that can be obtained from:

$$
R(q)=-M q^{2}\left(\left.\frac{\partial^{2} f(c)}{\partial c^{2}}\right|_{c_{0}}+2 \kappa q^{2}\right)
$$

should produce a straight line for the early stages of SD. In intermediate stages the scattering intensity continues to increase but at a slower rate than in early stages of phase separation by $\mathrm{SD}$ and $q$ decreases and $\lambda$ (wavelength) increases. The relation between $q$ and $\lambda$ is:

$$
q=\left(\frac{4 \pi}{\lambda}\right) \sin \left(\frac{\theta}{2}\right)
$$

where $\theta$ is the scattering angle. A typical plot of the scattering intensity in the early to the beginning of the intermediate stages for phase separation by SD is shown in Figure 5.13 where the scattering intensity is increasing with time. As mentioned before, $A^{*}\left(\mathbf{k}^{*}, t^{*}\right)$ is the Fourier transform of the composition fluctuations, $c(\mathbf{r}, t)$. Following equation (5.1) at the early and intermediate stages of phase separation by SD, the concentration fluctuations in Fourier space, $A^{*}\left(\mathbf{k}^{*}, t^{*}\right)$ should then describe the same characteristics as discussed above regarding the development of the scattering data (Figure 5.13). Thus, the structure factor, 
$S^{*}\left(\mathbf{k}^{*}, t^{*}\right)$ and the wave (position) vector, $\mathbf{k}^{*}$ closely are similar to the scattered light intensity, $I(q, t)$ and wave number, $q$ in the small angle light scattering experiments performed for the phase separation studies in polymer mixtures, respectively.

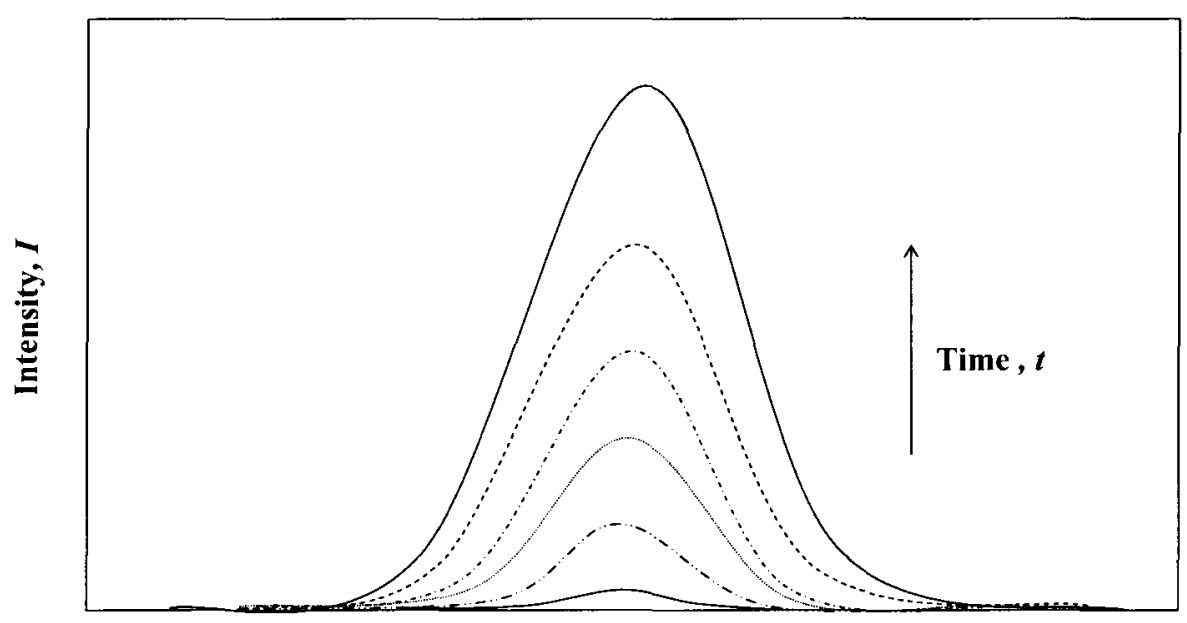

Wave Number, $q$

Figure 5.13: Typical light scattering profile showing the evolution of phase separation by SD in the early to the beginning of the intermediate stages. Each profile represents the intensity at a certain time $t$. The increase in time is indicated by the arrow in the up direction. The scattering intensity is increasing with time.

The dimensionless structure factor $S^{*}\left(\mathbf{k}^{*}, t^{*}\right)$ is calculated simply by taking the squares of the magnitude of the Fourier transform, $A^{*}\left(\mathbf{k}^{*}, t^{*}\right)$ of the concentration fluctuations in the system. In consequence, from equation (5.1), the structure factor can be expressed as: 
$\left.S^{*}\left(\mathbf{k}^{*}, t^{*}\right)=\left\|A^{*}\left(\mathbf{k}^{*}, t^{*}\right)\right\|^{2}=\| \sum_{m=0}^{N=1} \sum_{n=0}^{N=1}\left[c(\mathbf{r}, t)-c_{\circ}\right] e^{\left(\frac{2 \pi i}{n}\left(m k_{1}+n k_{2}\right)\right.}\right) \|^{2}$

where, $c(\mathbf{r}, t)$ is the concentration of solvent at node $(m, n), c_{\circ}$ is the mean solvent concentration, and $\mathbf{k}^{*}$ is the two-dimensional position vector, $\left(\mathbf{k}_{1}, \mathbf{k}_{2}\right)$ in Fourier space. To ensure that the numerical work in this study is in agreement with the known evolution of scattering profiles as associated to the structure factor, the dimensionless structure factor was calculated at different dimensionless times. Then a small algorithm was developed in MATLAB to determine the Fast Fourier Transform of the simulation results at a specific time. Taking the square of the magnitude of the Fast Fourier Transform results leads to determine the value of the structure factor.

Table 5.2: Dimensionless parameters used in the simulation for the analysis of structure factor evolution (fist three cases where $D^{*}=5000$ ) and the investigation of thermal diffusion coefficient effect on TIPS in binary polymer solutions with $D^{*}=8000$.

\begin{tabular}{|c|c|c|c|c|c|}
\hline Quench & $c_{\circ}^{*}$ & $D^{*}$ & $T^{*}$ & $N_{1}$ & $N_{2}$ \\
\hline A to B & 0.545 & 5000 & 0.650 & 1 & 10 \\
\hline A to C & 0.545 & 5000 & 0.625 & 1 & 10 \\
\hline A to D & 0.545 & 5000 & 0.560 & 1 & 10 \\
\hline A to D & 0.545 & 8000 & 0.560 & 1 & 10 \\
\hline
\end{tabular}


The dimensionless parameters used in the first three simulation cases listed in Table 5.2 are considered for the analysis of structure factor evolution during double phase separation with $D^{*}=5000$. The last case investigates the effect of thermal diffusion coefficient on the phase separation mechanism with $D^{*}=8000$. As already noted, the growth process can be quantified by determining the evolution of the structure factor with quench time. Figure 5.14 shows the evolution of the dimensionless structure factor as a function of dimensionless wave number, $\mathbf{k}^{*}$ and dimensionless time for the off-critical shallow double quench case (A to B) with $D^{*}=5000$ where the phase separation is followed by NG mechanism.

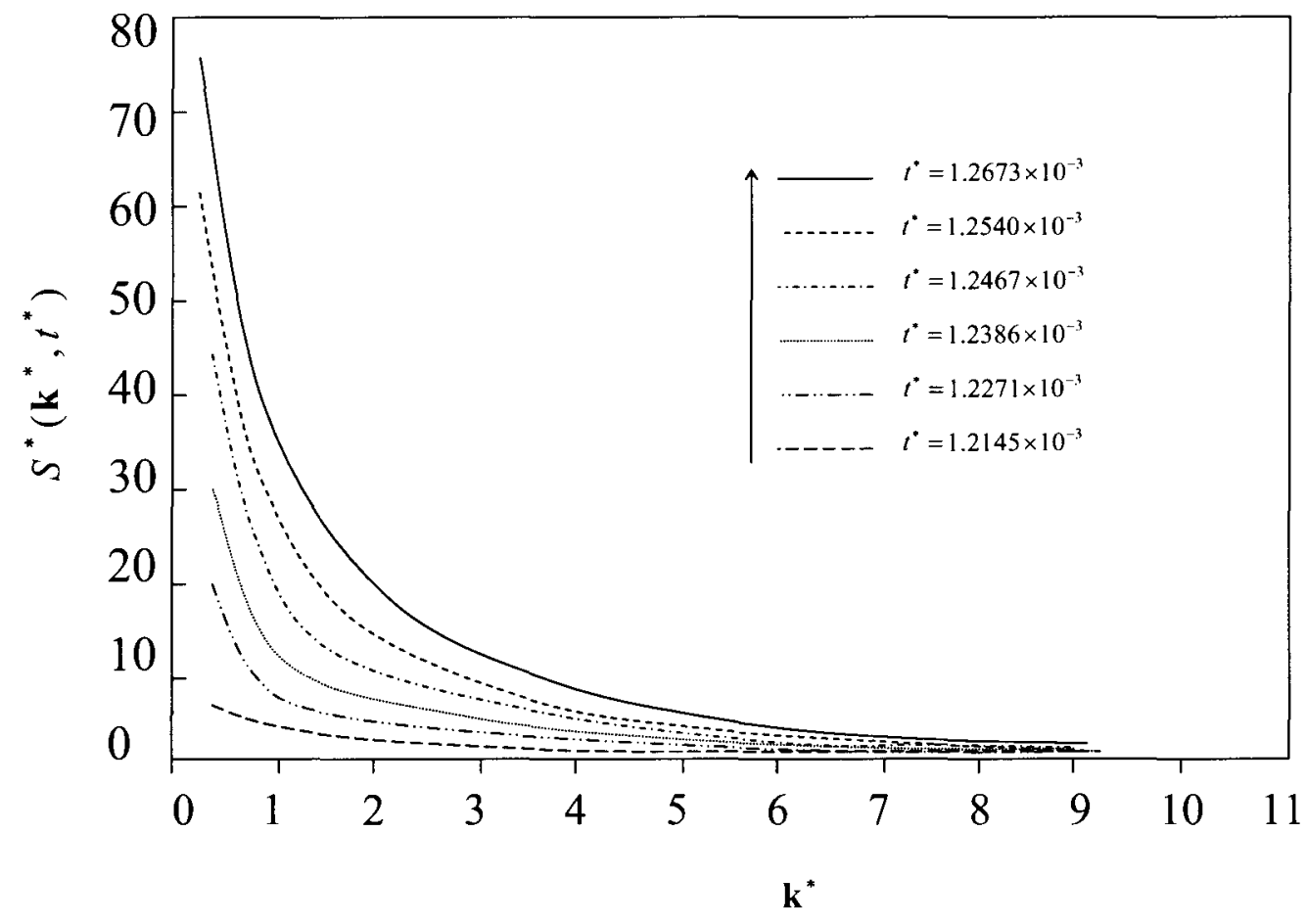

Figure 5.14: The evolution of the dimensionless structure factor for off-critical shallow double quench (A to B) at different dimensionless times within NG region. 
As observed in Figures 3.11 and 3.12, the spinodal decomposition process leads to a maximum in the angular variation of the scattered light intensity. The nucleation and growth mechanism is characterized by the absence of such a maximum and the continual decrease of the scattered light intensities with increasing angles [93]. For systems undergoing spinodal decomposition, dimensionless structure factor increases exponentially with time in the early stages of phase separation and displays a maximum that also grows with time. For systems undergoing nucleation and growth, dimensionless structure factor increases with time without displaying this maximum (Figure 5.14). The structure factor then begins to slow down as it approaches the beginning of the intermediate stages for both SD and NG mechanisms. Also during the early to the beginning of the intermediate stages, the wave number is constant. Thus, the evolution of the dimensionless structure factor for off-critical shallow double quench case show the same trends that have been reported both in experimental $[93,94]$ and numerical work $[70,134,159]$.

\subsection{Structure Factor Evolution in Deep Double Quench}

This section is split into two parts to describe the evolution of the dimensionless structure factor after the second quench: i) to $T_{3}{ }^{*}=0.625$ and ii) $T_{4}{ }^{*}=0.56$ for the off-critical deep quench cases. The dimensionless structure factor is calculated in the same way as described before for the shallow double quench case. The evolution of the dimensionless structure factor for case A to C is shown in Figure 5.15 and for case A to D is shown in Figure 5.16. 
Experimentally, after the second deep quench at a certain domain size formed from the first quench by NG, there appeared a second small peak that grew with time in the light scattering profile [134,136]. This observation from the light scattering data was a result of the formation of cavity domains. The structure factor from the numerical work also shows the appearance and growth of this small peak. Henderson and Clarke [168] have observed the appearance and growth of a second small peak in the evolution of the structure factor after the second quench at the late stages of phase separation by SD in their numerical study.

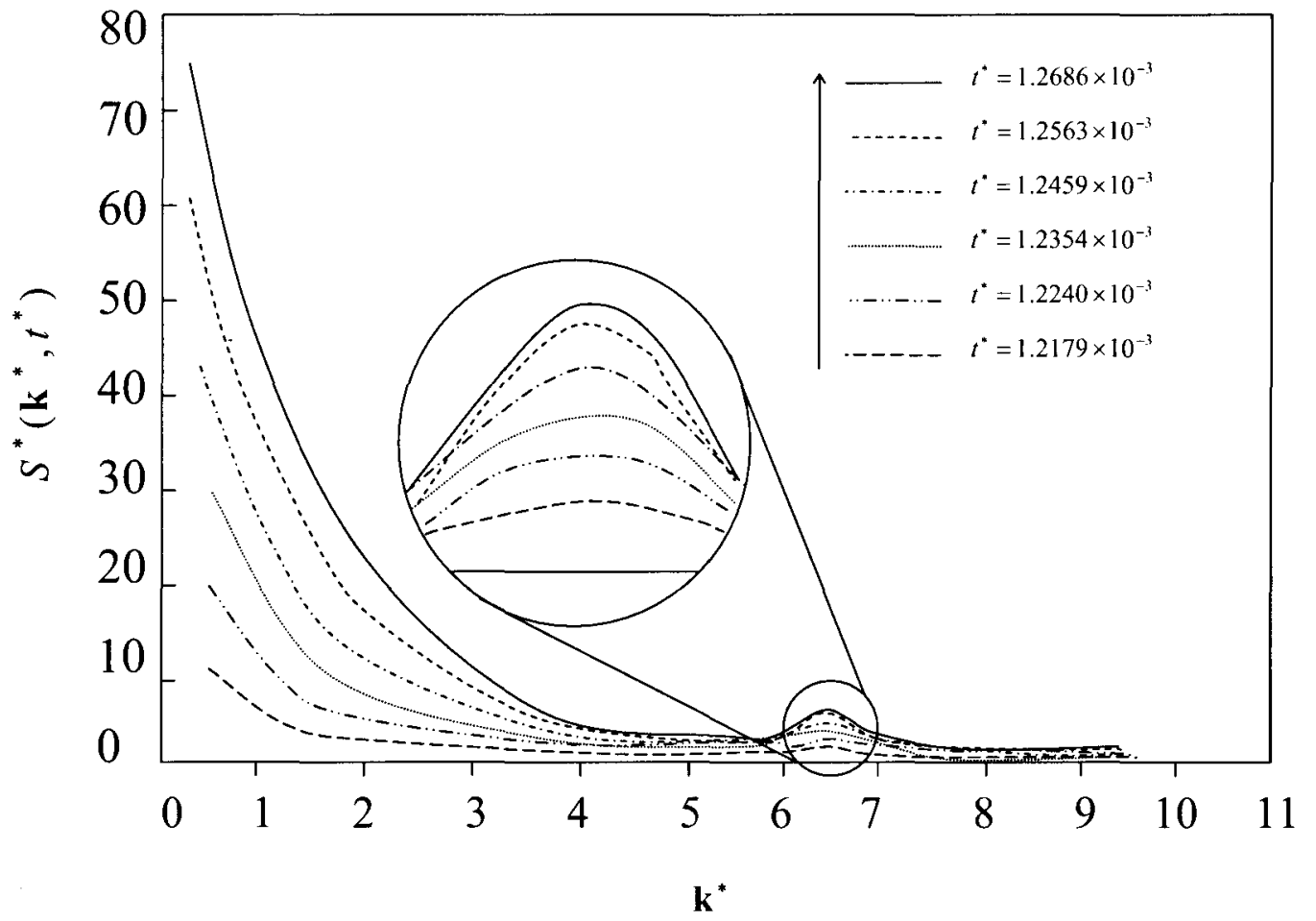

Figure 5.15: The evolution of the dimensionless structure factor for off-critical deep double quench ( $\mathrm{A}$ to $\mathrm{C}$ ) at different dimensionless times within NG region. The second broad peak is caused by the cavity formation inside the solvent droplets. 
This is the basis of comparison for the numerical results presented in this section since the structure factor at the start of the second quench in their simulation is developed as a weak but distinct shoulder [168] representing the secondary structure. As time increases, the magnitude of the secondary peak also increases. This is because the number of droplets increases with time as the phase separation of solvent and polymer continues [168].

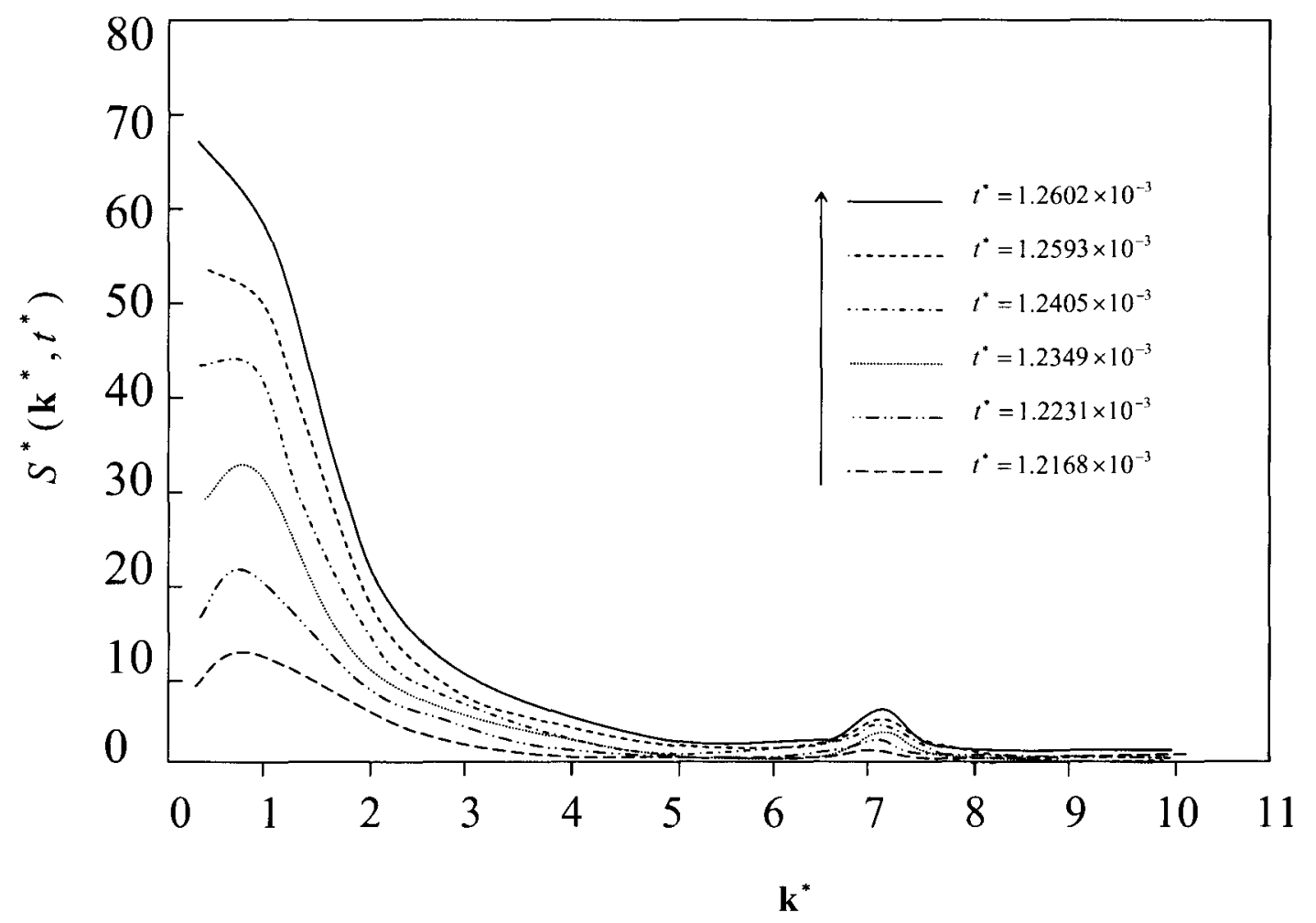

Figure 5.16: The evolution of the dimensionless structure factor for off-critical deep double quench (A to D) at different dimensionless times within NG (solvent) and SD (polymer) region. The second broad peak is caused by the cavity formation inside the solvent droplets. The value of dimensionless structure factor tends to increase exponentially with time in the early stages of phases separation by SD. 
Eventually the peak stops increasing in magnitude and decays as the secondary structure disappears. But in Figure 5.15 the secondary peak, which is created by imposing the second deep quench, will remain weak and does not grow with time. This is due to the fact that the second quench (A to C) although is deep but is still in the metastable region where the phase separation of both polymer and solvent would follow nucleation and growth mechanism. In Figure 5.16, the second deeper quench (A to D) thrusts the polymer into the unstable region and the solvent still within the metastable region (Figure 5.4). Both polymer and solvent follow the phase separation mechanism independently by spinodal decomposition and nucleation and growth respectively. Figure 5.16 shows an exponential trend at the beginning similar to the structure factor evolution resulted from SD mechanism which is in agreement with experimental results of Kiran and Liu [93,94] and numerical results of Henderson and Clarke [168].

\subsection{Structure Factor Growth from NG to SD}

From experimental observation of the early stages of SD phase separation by Hashimoto et $a l$. [103] the growth of the scattering intensity is exponential in the early stage and taking the natural $\log$ of these values should produce a straight line. In the later stages of phase separation by SD mechanism, the growth rate slows down and nonlinear effects take place. This was thought to be due to the coarsening of the mixture. Taking the natural $\log$ of equation (5.2) gives:

$\ln I(q, t)=2 R(q) t+\ln I(q, t=0)$ 
Equation (5.5) is the equation of a straight line. Figure 5.17 shows the natural log plots of the maximum value of the dimensionless structure factor versus dimensionless time for the offcritical shallow (A to B), deep (A to C) and deeper (A to D) double quench case respectively with $D^{*}=5000$. At the early stages of phase separation by NG in shallow and deep quench cases ( $A$ to $B$ and $C$ ) the initial increase is linear and gradually slows down into the beginning of the intermediate stage.

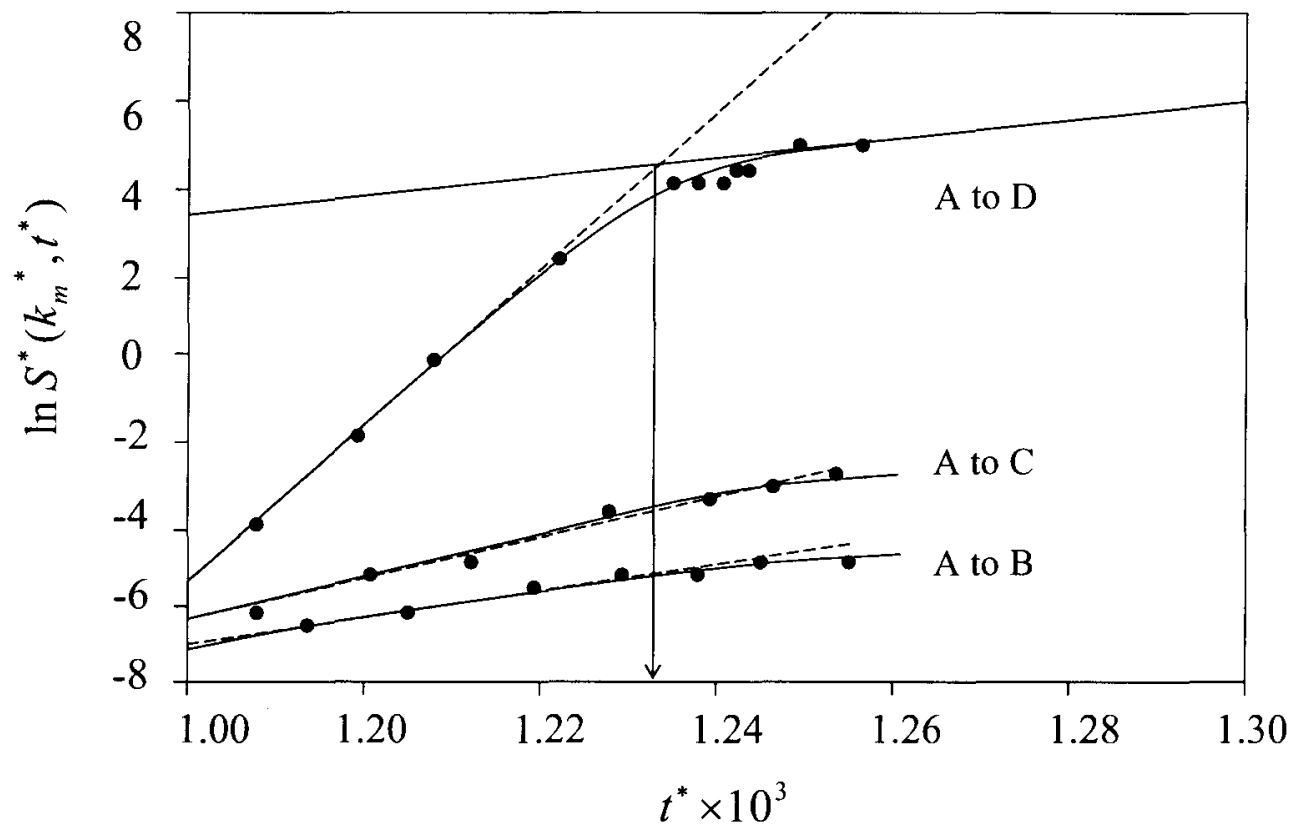

Figure 5.17: The evolution of the dimensionless maximum structure factor versus dimensionless time for shallow (A to B), deep (A to $C$ ) and deeper ( $A$ to $D)$ double quench cases respectively. The natural logarithm of the maximum structure factor increases linearly (non-exponentially) with time for $\mathrm{A}$ to $\mathrm{B}$ and $\mathrm{C}$ quenches which is considered to be typical of NG mechanism while, it increases exponentially with time for $A$ to $D$ case which is the characteristic of SD mechanism. Transition time (downward arrow) is at $1.2315 \times 10^{-3}$. 
The natural logarithm of the maximum structure factor increases non-exponentially with time for $\mathrm{A}$ to $\mathrm{B}$ and $\mathrm{C}$ quenches which is considered to be typical of NG mechanism. This is consistent with experimental work of Hashimoto et al. [159], Inoue et al. [60] and Vekilov et al. [89] and numerical studies of Henderson and Clarke [168]. At the early stages of phase separation by SD mechanism the natural logarithm of the maximum structure factor increases exponentially with time for A to D case which is the characteristic of SD mechanism. The transition point occurs where the two tangent lines intersect. The dimensionless transition time, $t_{i}{ }^{*}$, is defined as the time at which phase separation moves away from the early stage dynamics and enters into the beginning of the intermediate stages. From the plot of the maximum values of the dimensionless structure factor at given dimensionless times (Figure 5.17), the dimensionless transition time can be obtained by making two tangent lines where the change in the growth rate is obvious. In Figure 5.17, the two tangent lines are drawn and the point of intersection is where the transition time is located. The intersection of the two straight lines drawn on each side of the curve represents the transition point between the early and the intermediate stages of the SD mechanism and the dimensionless time indicated by the downward arrow gives the dimensionless transition time.

\subsection{Effect of Diffusion Coefficient}

As already mentioned dimensionless diffusion coefficient $D^{*}$, controls the amount and rate of phase separation. When $D^{*}$ increases the amount and rate of phase separation increase as well. 

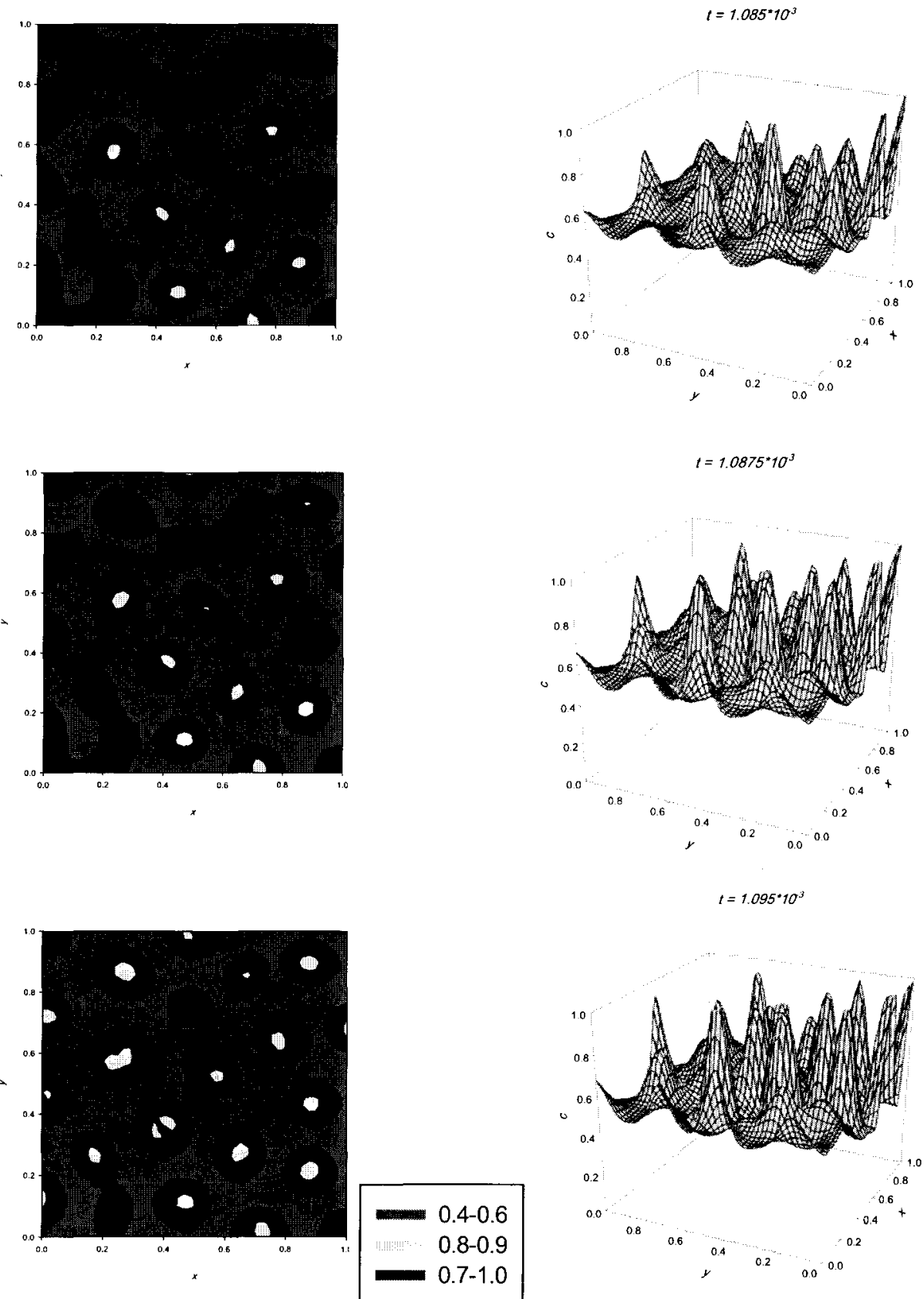

Figure 5.18: Dimensionless 2D contour spatial concentration profiles for $c^{*}\left(x^{*}, y^{*}\right)$ (left column) and 3D concentration fluctuation patterns (right column) for an off-critical deep double quench case (A to $D$ ) at the following dimensionless times respectively: $t^{*}=1.0852 \times 10^{-3}, t^{*}=1.0875 \times 10^{-3}$ and $t^{*}=1.0956 \times 10^{-3}$ where $D^{*}=8000$. 
Figure 5.18 illustrates the same quench path from A to D. Similar to Figure 5.8 the cavities are formed inside the solvent droplets but the diffusion coefficient is increased from 5000 to 8000. Figure 5.18 proves that the dimensionless diffusion coefficient controls the characteristic length scale and time scale of the pattern formed during phase separation. When the diffusion is increased the number of solvent droplets in polymer matrix and the phase separation rate are increased consequently. Diffusion coefficient affects the amount of phase separation, and the transition time for macroscopic phase separation to first occur [32]. As diffusion coefficient increases, there is more phase separation and the transition time decreases. This should be expected since phase separation occurs at a much faster rate with increasing diffusion and thus, should reach the beginning of the intermediate stage much faster. Diffusion coefficient, however, does not affect the type of morphology formed during the phase separation process.

Differences between the simulations arise due to the numerical difficulty of capturing the true early stages. In all cases the secondary structure reaches a maximum before the morphology relaxes back to the initial structure, but with a greater difference in composition between the two phases. As in experiments, [86-91] the secondary structure is absorbed back into the primary structure while the primary structure coarsens. However, we do not observe coarsening of the secondary structures; this is probably due to the initial primary structure not having a significantly greater length scale than the secondary, although another possibility is the neglect of hydrodynamic effects that has been considered in the simulations. Achieving such a wide range of length scales with the resultant increase in time scales is beyond the scope of this study. 


\section{Chapter 6}

\section{Conclusion}

In this thesis, we studied using mathematical modeling and computer simulation the morphology development and evolution during the two-step phase separation phenomenon in an asymmetric polymer solution. Many properties of polymer solutions can be determined from computer simulation, as the simulation route provides an opportunity to test the assumptions made in the theory and analysis. Although a large number of parameter values were used in our comprehensive numerical study (appendix A), Table 5.1 lists only a sample of parameter values, which are sufficient to achieve the objective stated above. For this study we used three different values for the dimensionless diffusion coefficient $D^{*}$ (appendix A). The initial quench is to $T_{1}$ followed by a second quench after some elapsed time to $T_{2}$ for a shallow quench or $T_{3}$ and $T_{4}$ for a deeper quench. It should be noticed that the transition time decreases as $D^{*}$ increases. This is to be expected since phase separation occurs at a greater rate with increasing $D^{*}$ and, therefore, should reach the beginning of the intermediate stage much faster. After the second quench, the growth of the dimensionless structure factor continues as if in the early stages of phase separation and proceeds to take the same course as a single quench into the intermediate stage. We then studied the formation of the cavities which are often found in asymmetric solutions. The formation of the cavities, growing inside 
the solvent droplets, is divided into three regimes: (a) early (cavity formation), (b) intermediate (cavity growth) and (c) late (cavity shrinkage) regimes. The formation of the cavity is generally associated with a high rate of polymer diffusion. In early regime when the rate of polymer diffusion inside the solvent droplets is high, a cavity structure will be obtained. The formation of cavities can be also explained as the result of changing interface diffusion during the second quench. The interface diffusion of the solvent-rich phase will decrease, while the interface diffusion of the polymer phase increases. At this point cavity formation is easily initiated till the interface diffusions of solvent and polymer become equal. In intermediate regime, growth of cavities will occur through more diffusion of the polymer into the solvent phase. Polymer molecules outside of the solvent droplets will reduce their chemical potential. Eventually, the solvent droplets will contain so much of the cavity. In the late regime the deliverance of the polymer molecules from each droplet into the polymer matrix just outside the solvent droplet occurs by diffusion mechanism. This transfer will occur by creeping. Eventually, complete cavity removal will take place. Cavities will diminish when the difference in chemical potential of the solvent droplets in the polymer solution and the polymer matrix is lowered. When the phase separation has proceeded through the diffusion of cavities from spherical solvent droplets into polymer-rich phase, the polymer matrix will gradually become more concentrated until it eventually solidifies. If the cavity is not given enough time to diffuse into the polymer through the solvent droplets, the final structure would be a continuous matrix of polymer with the typical sponge-like (coreshell) structure. In this case the cavities tend to accumulate inside the solvent droplet boundary, affecting the anchoring of the solvent and the optical homogeneity of the polymer matrix. 
However, we expect that this morphology of solvent droplets would improve the electrooptical properties such as the removal of the hysteresis effect and various optical properties can be obtained by introducing various types of solvent and by controlling the composition and properties of the matrix polymers. This work is of both academic and commercial interest. The motivation for this study was the need to understand and control the morphology formation and evolution in polymer solutions to fabricate functional polymeric materials with predefined specific material properties and characteristics. The model is able to reproduce recently reported experimental observations reported in the literature. This includes the observation that secondary phase separation occurs only if the second quench is sufficiently deep. Lastly, the numerical results indicate that the dimensionless diffusion coefficient and the quench depth can be used as a parameter to control the formation and evolution of the morphology during a double quench to tailor-make functional polymeric materials with predefined material properties and characteristics. The rate of phase separation and size of the phase-separated regions will decrease as $D^{*}$ increases. As well, phase separation occurs at a much faster rate with increasing diffusion reaching the beginning of the intermediate stage much faster. 


\section{References}

[1] J. W. Cahn, J. E. Hilliard. J. Chem. Phys. 28, 258 (1958).

[2] J. W. Cahn. J. Chem. Phys. 42, 93(1965).

[3] Y. C. Chou and W. I. Goldburg. Phys. Rev. A 20, 2105 (1979).

[4] N. C. Wong and C. M. Knobler. Phys. Rev. A 24, 3205 (1981).

[5] T. Hashimoto, M. Itakura and Shimazu N. J. Chem. Phys. 85, 6118 (1986).

[6] P. Guenoun, R. Gastaud, F. Perrot, and D. Beysens. Phys. Rev. A 36, 4876 (1987).

[7] H. Tanaka, T. Yokokawa, H. Abe, T. Hayashi, and T. Nishi. Phys. Rev. Lett. 65, 25, (1990).

[8] A. Onuki, Phys. Rev. Lett., 48, 753 (1982).

[9] F. S. Bates and P. Wiltzius, J. Chem. Phys., 91, 3258 (1989).

[10] H. Tanaka and T. Sigehuzi, Phys. Rev. Lett., 75, 874 (1995).

[11] P. Guenoun, B. Khalil, D. Beysens, Y. Garrabos, F. Kammoun, B. LeNeindre, and B. Zappoli, Phys. Rev. E 47, 1531 (1993).

[12] K. D. Lee, Philip K. Chan, Xianshe Feng. Macromol. Theory Simul., 11, 996, (2002).

[13] K. D. Lee, Philip K. Chan, Xianshe Feng. Macromol. Theory Simul., 12, 413, (2003)

[14] K. D. Lee, Philip K. Chan, Xianshe Feng. Chem. Eng. Sci., 59, 1491 - 1504, (2004).

[15] Bai Tao Jiang, Philip K. Chan. Macromol. Theory Simul., 16, 690-702, (2007).

[16] Copetti and C.M. Elliott, Mater. Sci. Tech., 6, 273 (1990).

[17] J.L. West, Mol. Cryst. Liq. Cryst., 157,427 (1988).

[18] G.W. Smith and N.A. Vaz. Liq. Cryst., 3, 543 (1988). 
[19] I. C. Henderson. N. Clarke. The Jour. of Chem. Phy., 123, 144903, (2005).

[20] J. D. Gunton, M. San Miguel, and P. Sahni, in Phase Transition and Critical

Phenomena, edited by C. Domb and J. H. Lebowitz. London., Vol. 8 (1983).

[21] J.D. Gunton and M. Droz, Lecture Notes in Physics, edited by H. Araki, SpringerVerlag, New York, Vol. 183 (1983).

[22] H. Tanaka, Phys. Rev. Lett. 70, 2770 (1993); J. Phys.: Condens. Matter 13, 4637 (2001).

[23] T. Nihei, J. Nephew, and S. A. Carter 80,15, 3276 (1998).

[24] T. Kyu, M. Mustafa, J. C. Yang, J. Y. Kim, and P. Palffy-Muhoray, Stud. Polym. Sci., $11,245(1992)$.

[25] P. K. Chan, Tuyet L. Tran, D. Rousseau. Comput. Mater. Sci., 37, 328, (2006).

[26] H. Tanaka, J. Chem. Phys., 105, 10099 (1996).

[27] A. Onuki, Phase Transition Dynamics. Cambridge University Press, Cambridge, England., (2002).

[28] M. Joshua, W. I. Goldburg, and A. Onuki, Phys. Rev. Lett., 54, 1175 (1985).

[29] H.G. Craighead, J. Cheng, and S. Hackwood, Appl. Phys. Lett., 40, 22 (1982).

[30] P. K. Chan, A. D. Rey, Macromol. Theory Simul., 4, 873, (1995).

[31] P. K. Chan, A. D. Rey, Comput. Mater. Sci. 3, 377, (1995).

[32] P. K. Chan, A. D. Rey, Macromolecules 29, 8934, (1996).

[33] P. K. Chan, A. D. Rey, Macromolecules 30, 2135, (1997).

[34] P. K. Chan, A. D. Rey, Macromolecules 4, 2135. 873, (1995).

[35] J.L. Fergason, SID Digest, 16,68 (1985). P.S. Drzaic, Liquid Crystal Dispersions, World Scientific, Singapore (1995).

[36] J.L. Fergason, U.S. Patent ., 4,435,047 (1990). 
[37] Binder, K. J. Chem. Phys., 79, 6387, (1983).

[38] C. Hilsum, U.K. Patent 1,442,360 (1976).

[39] Rogers, T. M.; Elder, K. R.; Desai, R. C. Phys. Rev. B 1988, 37, 9638.

[40] H. Tanaka, T. Yokokawa, H. Abe, T. Hayashi, and T. Nishi., Phys. Rev. Lett., 65, 25 (1990).

[41] A. Chakrabarti, R. Toral, J. D. Gunton, M. Muthukumar. Phys. Rev. Lett., 63, 2072, (1989).

[42] A. Chakrabarti, J. D. Gunton, Phys. Rev. B, 37, 3798, (1988).

[43] K. Binder, Phy. Rev., 29, 1, (1984).

[44] M. Takenaka, T. Hashimoto., Phys. Rev. E 48, 647, (1993).

[45] P. Chan, PhD thesis, McGill University, Montreal, Canada., (1997).

[46] C. Huang, M. O. Cruz, Macromolecules, 27, 4231, (1994).

[47] H. Matsuyama, M. Teramoto, S. Kudari and Y. Kitamura, Jour. of App. Poly. Sci., 82, $169,177(2001)$.

[48] H. Matsuyama, T. Maki, M. Teramoto, M. and K. Asano., Jour. of Memb. Sci., 204, 323 328 (2002).

[49] Matsuyama, H., Kim, M.-m., and Lloyd, D. Journal of Membrane Science 204, 413-419 (2002).

[50] P. G. deGennes, J. Chem. Phys. 1980, 72, 4756.

[51] A. C. Balazs, V. Ginzburg, F. Qui, G. Peng, D. Jasnow, J. Chem. Phys. 104, 3411, (2000).

[52] T. Ohnaga, T. Inoue. Jour. of Poly. Sci.: Part B: Poly. Phy., Vol. 27, 1675-1689 (1989).

[53] N. Clarke, Macromolecules 37, 1952-1959, (2004). 
[54] N. Clarke, Phys. Rev. Lett., 89, 215506, (2002).

[55] M. Hayashi, H. Jinnai, T. Hashimoto, J. Chem. Phys., 113, 3414, (2000).

[56] P. Pincus, J. Chem. Phys. 75, 1996 (1981).

[57] K. Kwak, M. Okada, T. Chiba, T. Nose, Macromolecules, 26, 4047, (1993).

[58] M. Fialkowski, R. Holyst, J. Chem. Phys. 117, 1886, (2002).

[59] J. Tao, M. Okada, T. Nose, T. Chiba. Polymer Vol. 36 No. 20, pp. 3909-3917, 1995

[60] Y. Yang, H. Fujiwara, T. Inoue, T. Chiba. Polymer Vol. 39 No. 13, pp. 2745-2750, 1998

[61] Cook, H. E. Acta Metall. 1970, 18, 297.

[62] Glotzer, S. C. Annu. Rev. Comput. Phys. 1995, 2, 1.

[63] [44] Langer, J. S. Acta Metall. 1973, 21, 1649.

[64] J. K. Kim, K. Taki, M. Ohshima Langmuir, 2007, 23 (24), 12397-12405

[65] A. Onuki, Phase Transition Dynamics. Cambridge University Press, Cambridge, 2002.

[66] K. D. Kwak, M. Okada, T. Chiba, and T. Nose, Macromolecules 26, 4047, 1993.

[67] M. Hayashi, H. Jinnai, and T. Hashimoto, J. Chem. Phys. 112, 6897, 2000

[68] B. M Forrest and D. W. Heermann. J Phys II France (1991) 909-919 AOUT 1991, 909

[69] 2. I.-P. Chen and Y.-D. Lee, Polymer, 36, 55 (1995).

[70] Chakrabarti, A.; Toral, R.; Grunton, J. D.; Muthukumar, M. J. Chem. Phys. 1990, 92, 6899 .

[71] Brown, G.; Chakrabarti, A. J. Chem. Phys. 1993, 98, 2451.

[72] G. Brown and A. Chakrabarti, Phys. Rev. E, 4S, 3705 (1993).

[73] I. Podariu, A. Chakrabarti. Jour. of Chem. Phy. 126, 154509, 2007

[74] D. A. Huse, Phys. Rev. B 34, 7845,1986.

[75] J.W. Cahn, J. Chern. Phys. 66, 3667 (1977). 
[76] For a review, see D. Jasnow, Rep. Prog. Phys. 47, 1059 (1984); P.G. de Gennes, Rev. Mod. Phys. 57,827 (1985).

[77] Y. J. Jeon, Y. Bingzhu, J. T. Rhee, D. L. Cheung, M. Jamil Macromol. Theory Simul. $2007,16,643-659$

[78] L. T. Yan, J. Li, F. Zhang, X. M. Xie. J. Phys. Chem. B 2008, 112, 8499-8506

[79] P. Guenoun et al., Phys. Rev. Lett. 65, 2406 (1990).

[80] R.A.L. Jones et al., Phys. Rev. Lett. 66, 1326 (1991).

[81] P. Wiltzius and A. Cumming, Phys. Rev. Lett. 66, 3000 (1991).

[82] A. Cumming et al., Phys. Rev. A 45, 885 (1992).

[83] M. Fiałkowski, R. Hołyst. Jour. of Chem. Phy. 117, 4, 2002

[84] H. Tanaka, Phys. Rev. Lett. 70, 2770 (1993).

[85] E.D. Siggia, Phys. Rev. A 20, 595 (1979).

[86] H. Tanaka, Phys. Rev. E 47, 2946 (1993).

[87] H. Tanaka, Phys. Rev. Lett. 81, 2 (1998).

[88] P. Guenoun et al., Phys. Rev. A 36, 4876 (1987).

[89] Mrinal Shah, Oleg Galkin, and Peter G. Vekilov. Jour. of Chem. Phy. 121, No. 15, 2004

[90] Hashimoto, T.; Hayashi, M.; Jinnai, H. J. Chem. Phys. 2000, 112, 6886.

[91] Hayashi, M.; Jinnai, H.; Hashimoto, T. J. Chem. Phys. 2000, 112, 6897.

[92] F.8. Bates and P. Wiltzius, J. Chern. Phys. 91, 3258 (1989).

[93] Ke Liu and Erdogan Kiran. Macromolecules 2001, 34, 3060-3068

[94] Ke Liu and Erdogan Kiran. Journal of Supercritical Fluids 16 (1999) 59-79

[95] H. Tanaka, Phys. Rev. Lett. 72,23 (1994).

[96] H. Tanaka, T. Sigehuzi. Phys. Rev. E 70, 051504 (2004) 
[97] T. Hashimoto et al., J. Chern. Phys. 85, 6773 (1986).

[98] P. C. Hohenberg and B. I. Halperin, Rev. Mod. Phys. 49, 435 (1976).

[99] Comp. and Theo. Poly. Sci., Vol.11, No. 6, 2001, 445-458(14) Nakazawa H.; Fujinami

S.; Motoyama M

[100] E. D. Siggia, Phys. Rev. A 20, 595 (1979).

[101] Y. C. Chou and W. I. Goldburg, Phys. Rev. A 20, 2105 (1979); N. C. Wong and C. M. [102] Knobler, Phys. Rev. A 24, 3205 (1981).

[103] T. Hashimoto et al., J. Chem. Phys. 85, 6118 (1986).

[104] P. Guenoun et al., Phys. Rev. A 36, 4876 (1987)

[105] F. S. Bates and P. Wiltzius, J. Chem. Phys. 91, 3258 (1989).

[106] A. Shinozaki and Y. Oono, Phys. Rev. E 48, 2622 (1993).

[107] T. Koga and K. Kawasaki, Physica (Amsterdam) 196A, 389 (1993).

[108] See, e.g., H. Furukawa, Phys. Rev. E 55, 1150 (1997).

[109] P. Wiltzius and A. Cumming, Phys. Rev. Lett. 66, 3000 (1991).

[110] A. Cumming et al., Phys. Rev. A 45, 885 (1992).

[111] B. Q. Shi et al., Phys. Rev. Lett. 70, 206 (1993).

[112] 41. P.J. Flory, Principles of Polymer Chemistry, Cornell University Press, Ithaca (1953).

[113] J.M.G. Cowie, Polymers: Chemistry and Physics of Modern Materials, 2nd edition, Chapman and Hall, New York (1991).

[114] Phys. Rev. E 51, 1313 (1995).

[115] B. Chu et al., J. Am. Chern. Soc. 90, 3402 (1968); E. Gulari et al., J. Chern. Phys. 56, $6169(1972)$. 
[116] S. M. Troian, Phys. Rev. Lett. 71, 1399 (1993).

[117] H. Tanaka, Phys. Rev. Lett. 72, 1702 (1994).

[118] Dynamics of Ordering Process in Condensed MaUer, edited by S. Komura and H.

Furukawa (Plenum, New York, 1987).

[119] H. Tanaka et al., Phys. Rev. Lett. 65, 3136 (1990).

[120] K. Binder, Phys. Rev. B 15,4425 (1977).

[121] G. Brown and A. Chakrabarti, J. Chern. Phys., 98, 2451 (1993).

[122] G. Brown and A. Chakrabarti, Phys. Rev. E, 48,3705 (1993).

[123] R. Ruiz, Phys. Rev. A 26, 2227 (1982).

[124] A. Onuki, Prog. Theor. Phys. 66, 1230 (1981).

[125] A. Onuki, Phys. Rev. Lett. 48, 753 (1982).

[126] A. Onuki, Prog. Theor. Phys. 67, 768 (1982); 67, 787 (1982); 67,1740 (1982).

[127] M. Joshua et al., Phys. Rev. Lett. 54, 1175 (1985).

[128] D. Beysens and F. Perot, J. Phys. (Paris) Lett. 45, L31 (1984).

[129] D. Jasnow et al., Phys. Rev. A 23,3192 (1981).

[130] H. Tanaka, Polym. Prep. Jpn. 40,766 (1991).

[131] M. Okada et al., Polym. Prep. Jpn. 40, 767 (1991).

[132] K. Binder and D. Stauffer, Phys. Rev. Lett. 33, 1006 (1974).

[133] K. Binder and D. Stauffer, Adv. Phys. 25, 343 (1976).

[134] D.J. Hourston, H.X. Zhang, M. Song, M. Pollock, A. Hammiche. Thermochimica Acta 294 (1997) 23-31

[135] K. Zhao, P. Sun, D. Liu, G. Dai. Euro. Poly. Jour. 40 (2004) 89-96

[136] K. Ishizu Prog. Polym. Sci., Vol. 23, 1383-1408, 1998 
[137] J. J.M. Halls, C. A. Walsh, N. C. Greenham, E. A. Marseglia, R. H. Friend, S.C.

Moratti, and A. B.Holmes, Nature (London) 376, 498 (1995).

[138] B. J. P. Jansen, S. Rastogi, H. E. H. Meijer, P. J. Lemstra Macromolecules 2001, 34, $3998-4006$

[139] Z. Huang, G. Chidichimo, A. Golemme, H. A. Hakemi, M. Santangelo. F. P. Nicoletta. Liquid Crystals, 1997, Vol. 23, No. 4, 519-524

[140] K. Binder, J. Non-Equilib. Thermodyn. 23, 1 (1998).

[141] M. Boltau, S. Walheim, J. Mlynek, G. Krausch, and U. Steiner, Nature (London) 391, 877 (1998).

[142] B. D. Ermi, G. Nisato, J. F. Douglas, J. A. Rogers, and A. Karim, Phys. Rev. Lett. 81, 3900 (1998).

[143] A.M. Higgins and R. A. L. Jones, Nature (London) 404, 476 (2000).

[144] A. Karim, J. F. Douglas, G. Nisato, D.-W. Liu, and E. J. Amis, Macromolecules 32, $5917(1999)$.

[145] I. M. Lifshitz and V. V. Slyozov, Phys. Chem. Solids 19, 35 (1961).

[146] K. D. Kwak, M. Okada, T. Chiba, and T. Nose, Macromolecules 26, 4047 (1993).

[147] H. Tanaka, Phys. Rev. E 47, 2946 (1993).

[148] M. Hayashi, H. Jinnai, and T. Hashimoto, J. Chem. Phys. 113, 3414 (2000).

[149] H. E. Cook, Acta Metall. 18, 297 (1970).

[150] J.W. Cahn and J. E. Hilliard, J. Chem. Phys. 28, 258 (1958).

[151] P.G. deGennes, J. Chem. Phys. 72, 4756 (1980).

[152] M. Okada, K. D. Kwak, and T. Nose, Polym. J. (Tokyo, Jpn.) 24, 215 (1992).

[153] K. Binder, J. Chem. Phys. 79, 6387 (1983). 
[154] S.C. Glotzer, Annu. Rev. Comput. Phys. 2, 1 (1995).

[155] P. J. Flory, Principles of Polymer Chemistry (Cornell University Press, Ithaca, N.Y., 1953).

[156] H. Tanaka, Phys. Rev. E 47, 2946 (1993).

[157] M. Graca, S. A. Wieczorek, and R. Holyst, Macromolecules 35, 7718 (2002); M.

Fialkowski and R. Holyst, J. Chem. Phys. 117, 1886 (2002).

[158] J. Tao, M. Okada, T. Nose, and T. Chiba, Polymer 36, 3909 (1995).

[159] T. Hashimoto, Kumaki, J. and Kawai, H., Macromolecules, Vol. 16, No. 4, (1983).

[160] A. Noviek-Cohen. Journal of Statistical Physics, Vol. 38, Nos. 3/4, 1985

[161] M. Hayashi, H. Jinnai, and T. Hashimoto, J. Chem. Phys. 112, 6897 (2000).

[162] M. Rullmann and I. Alig, J. Chem. Phys. 120, 7801 (2004).

[163] N. C. Wong and C. M. Knobler, Phys. Rev. Lett. 43,1733 (1979); 45, 498 (1979).

[164] N. C. Wong and C. M. Knobler, J. Chern. Phys. 69,725 (1978).

[165] M. Berggren, O. Inganas, G. Gustafsson, J. Rasmusson, M. R. Andersson, and T. Hjertberg, Nature (London) 372, 444 (1994).

[166] S. Puri and H. L. Frisch, J. Phys. Condens. Matter 9, 2109 (1997).

[167] B. P. Lee, J. F. Douglas, and S.C. Glotzer, Phys. Rev. E 60, 5812 (1999).

[168] I. C. Henderson and N. Clarke, Macromolecules 37, 1952 (2004).

[169] H. Tanaka, T. Hayashi, and T. Nishi, J. Appl. Phys. 59, 3627 (1986); 65, 4480 (1989).

[170]1. M. Lifshitz and V. V. Slyozov, J. Phys. Chern. Solids 19, 35 (1961).

[171] C. Wagner, Z. Elektrochem. 65, 581 (1961). 
[172] A.C. Edrington, A.M. Urbas, P. DeRege, C. X. Chen, T.M. Swager, N. Hadjichristidis, M. Xenidou, L. J. Fetters, J. D. Joannopoulas, Y. Fink, and E. Thomas, Adv. Mater. 13, 421 (2001).

[173] S. Puri, Y. Oono. J. Phys. A: Math. Gen. 21 (1988) L755-L762. Printed in the UK

[174] S. Puri, Y. Oono. 1987 Phys. Rev. Lett 58836.

[175] Jingzhi Zhu and Long-Qing Chen.1999 Phys. Rev. E 60,4.

[176] E. D. Siggia, Phys. Rev. A 20, 595 (1979).

[177] H. Tanaka, Phys. Rev. Lett. 72, 1702 (1994).

[178] C. Yeung. Phys. Rev. Lett. 61, 9 (1988).

[179] H. Tanaka, Phys. Rev. Lett. 72, 3690 (1994); Phys. Rev. E 51, 1313 (1995); H. Tanaka and T. Araki, Phys. Rev. Lett. 81, 389 (1998).

[180] N. C. Wong and C. M. Knobler, J. Chem. Phys. 69, 725 (1978).

[181] D. M. Koenhen, M. H. V. Mulder, and C. A. Smolders, Journal of Applied Polymer

Science; 21, 199-215 (1977)

[182] Tanaka, H. J. Phys.: Condens. Matter 2000, 12, R207.

[183] Peng, G.; Qiu, F.; Ginzburg, V. V.; Jasnow, D.; Balazs, A. C. Science 2000, 288, 1802. [184] de Gennes, P. G. Scaling Concepts in Polymer Physics; Cornell University Press:

Ithaca, NY, 1993.

[185] T. Sigehuzi and H. Tanaka, Phys. Rev. E 70, 51504, 2004. 


\section{Appendices}

A

Numerical parameter values featuring different system models with different average concentrations $\left(c_{\mathrm{o}}\right)$, mesh sizes, quench depths (temperatures), diffusions and transition times respectively.

\begin{tabular}{|c|c|c|c|c|c|c|c|c|c|c|c|}
\hline \multicolumn{12}{|c|}{$c_{\mathrm{o}}=0.41$} \\
\hline \multicolumn{6}{|c|}{ Mesh Size $=31 \times 31$} & \multicolumn{6}{|c|}{ Mesh Size $=20 \times 20$} \\
\hline \multicolumn{3}{|c|}{$\begin{array}{c}\text { Shallow Quench } \\
0.56 \text { to } 0.54\end{array}$} & \multicolumn{3}{|c|}{$\begin{array}{c}\text { Deep Quench } \\
0.6499 \text { to } 0.525\end{array}$} & \multicolumn{3}{|c|}{$\begin{array}{c}\text { Shallow Quench } \\
0.56 \text { to } 0.54\end{array}$} & \multicolumn{3}{|c|}{$\begin{array}{c}\text { Deep Quench } \\
0.6499 \text { to } 0.525\end{array}$} \\
\hline \multicolumn{12}{|c|}{ Diffusion } \\
\hline 1000 & 5000 & 8000 & 1000 & 5000 & 8000 & 1000 & 5000 & 8000 & 1000 & 5000 & 8000 \\
\hline$\stackrel{1}{0}$ & $\stackrel{1}{0}$ & 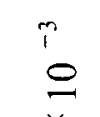 & 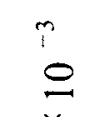 & $i$ & 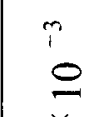 & $\stackrel{i}{0}$ & $\stackrel{1}{0}$ & $\stackrel{1}{1}$ & 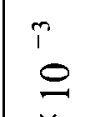 & $i$ & $\stackrel{1}{O}$ \\
\hline$x$ & $x$ & $x$ & $x$ & $x$ & $x$ & $x$ & $x$ & $\bar{x}$ & $x$ & $x$ & $\bar{x}$ \\
\hline 尔 & $\frac{\pi}{n}$ & $\stackrel{\curvearrowright}{\beth}$ & 志 & $\stackrel{\bar{m}}{\sim}$ & $\stackrel{\Omega}{\stackrel{\Omega}{I}}$ & 吉 & $\bar{n}$ & $\stackrel{\stackrel{2}{\beth}}{=}$ & $\underset{⿱}{\stackrel{\Xi}{+}}$ & $\frac{\hat{n}}{\sqrt{2}}$ & 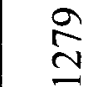 \\
\hline$\stackrel{?}{-}$ & $\because$ & $\because$ & $\stackrel{?}{-}$ & $\stackrel{?}{2}$ & $\because$ & $\dddot{?}$ & $\stackrel{\simeq}{\sim}$ & $\because$ & $\stackrel{?}{\sim}$ & $\stackrel{?}{\sim}$ & 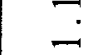 \\
\hline$\|$ & $\|$ & $\|$ & II & $\|$ & $\|$ & $\|$ & $\|$ & $\|$ & $\|$ & $\|$ & $\|$ \\
\hline${ }^{*}$ & ${ }^{*}{ }_{N}$ & ${ }^{*}{ }^{*}$ & ${ }^{*}$ & ${ }^{*} \sim$ & ${ }^{*} \sim$ & ${ }^{*} w$ & ${ }^{*} \sim$ & ${ }^{*} \sim$ & ${ }^{*} \sim$ & ${ }^{*} \sim$ & ${ }^{*} \sim$ \\
\hline
\end{tabular}




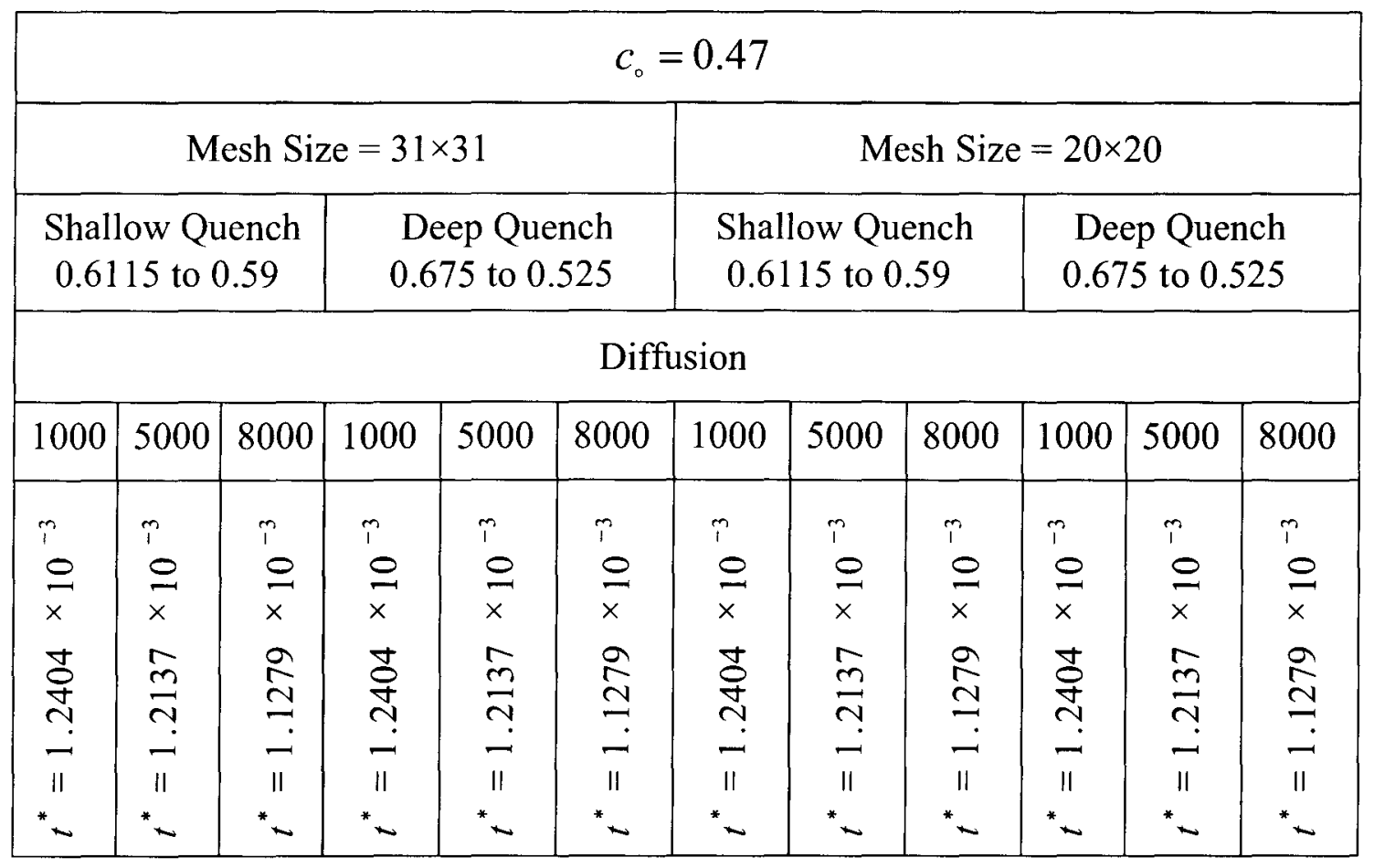

\begin{tabular}{|c|c|c|c|c|c|c|c|c|c|c|c|c|c|c|c|c|c|}
\hline \multicolumn{18}{|c|}{$c_{\circ}=0.53$} \\
\hline \multicolumn{9}{|c|}{ Mesh Size $=31 \times 31$} & \multicolumn{9}{|c|}{ Mesh Size $=41 \times 41$} \\
\hline \multicolumn{3}{|c|}{$\begin{array}{l}\text { Shallow Quench } \\
0.66 \text { to } 0.63\end{array}$} & \multicolumn{3}{|c|}{$\begin{array}{l}\text { Deep Quench } \\
0.66 \text { to } 0.58\end{array}$} & \multicolumn{3}{|c|}{$\begin{array}{l}\text { Deeper Quench } \\
0.66 \text { to } 0.525\end{array}$} & \multicolumn{3}{|c|}{$\begin{array}{c}\text { Shallow Quench } \\
0.66 \text { to } 0.63\end{array}$} & \multicolumn{3}{|c|}{$\begin{array}{l}\text { Deep Quench } \\
0.66 \text { to } 0.58\end{array}$} & \multicolumn{3}{|c|}{\begin{tabular}{|l} 
Deeper Quench \\
0.66 to 0.525
\end{tabular}} \\
\hline \multicolumn{18}{|c|}{ Diffusion } \\
\hline 1000 & 5000 & 8000 & 1000 & 5000 & 8000 & 1000 & 5000 & 8000 & 1000 & 5000 & 8000 & 1000 & 5000 & 8000 & 1000 & 5000 & 8000 \\
\hline$\frac{m}{x}$ & $\begin{array}{l}\tilde{i} \\
\stackrel{0}{x}\end{array}$ & $\frac{m}{x}$ & $\frac{1}{0}$ & $\frac{1}{x}$ & $\frac{m}{x}$ & $\stackrel{i}{i}$ & $\frac{1}{x}$ & $\frac{m}{x}$ & $\frac{p}{x}$ & $\frac{m}{x}$ & $\stackrel{i}{\hat{x}}$ & $\frac{m}{x}$ & $\frac{i}{x}$ & $\frac{i}{x}$ & $\frac{1}{x}$ & $\frac{m}{x}$ & $\frac{r}{x}$ \\
\hline ষ্ণ & m & 2 & I & m & 2 & t & $\hat{m}$ & 2 & I & $\hat{m}$ & 2 & t & m & 2 & 巳 & m & $\stackrel{2}{2}$ \\
\hline đั & $\bar{\imath}$ & $\simeq$ & $\stackrel{\pi}{4}$ & $\vec{\imath}$ & $\simeq$ & $\stackrel{\stackrel{\leftrightarrow}{\sim}}{\sim}$ & $\bar{\imath}$ & $\cong$ & 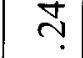 & $\vec{\imath}$ & $?$ & 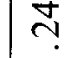 & $\bar{\top}$ & $\simeq$ & 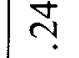 & $\bar{\imath}$ & 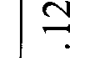 \\
\hline- & $\pi$ & $\pi$ & $\overline{7}$ & $\pi$ & $\bar{\pi}$ & $\pi$ & $\pi$ & $\pi$ & $\pi$ & $\pi$ & 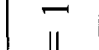 & 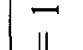 & $\pi$ & $\pi$ & $\pi$ & $\pi$ & $\pi$ \\
\hline & $*$ & $*$ & * & * & $*$ & * & " & $*$ & $*$ & $*$ & $*$ & $*$ & * & $*$ & $*$ & $*$ & ${ }^{*}$ \\
\hline
\end{tabular}




\begin{tabular}{|c|c|c|c|c|c|c|c|c|c|c|c|c|c|c|c|c|c|}
\hline \multicolumn{18}{|c|}{$c_{\circ}=0.585$} \\
\hline \multicolumn{9}{|c|}{ Mesh Size $=31 \times 31$} & \multicolumn{9}{|c|}{ Mesh Size $=20 \times 20$} \\
\hline \multicolumn{3}{|c|}{$\begin{array}{l}\text { Shallow Quench } \\
0.691 \text { to } 0.672\end{array}$} & \multicolumn{3}{|c|}{$\begin{array}{c}\text { Deep Quench } \\
0.691 \text { to } 0.6115\end{array}$} & \multicolumn{3}{|c|}{$\begin{array}{l}\text { Deeper Quench } \\
0.691 \text { to } 0.525\end{array}$} & \multicolumn{3}{|c|}{$\begin{array}{l}\text { Shallow Quench } \\
0.691 \text { to } 0.672\end{array}$} & \multicolumn{3}{|c|}{$\begin{array}{c}\text { Deep Quench } \\
0.691 \text { to } 0.6115\end{array}$} & \multicolumn{3}{|c|}{$\begin{array}{l}\text { Deeper Quench } \\
0.691 \text { to } 0.525\end{array}$} \\
\hline \multicolumn{18}{|c|}{ Diffusion } \\
\hline 1000 & 5000 & 8000 & 1000 & 5000 & 8000 & 1000 & 5000 & 8000 & 1000 & 5000 & 8000 & 1000 & 5000 & 8000 & 1000 & 5000 & 8000 \\
\hline$m$ & $\hat{\imath}$ & $\stackrel{\imath}{\imath}$ & i & $p$ & $\hat{\imath}$ & $?$ & $p$ & $p$ & $\hat{\imath}$ & $\hat{\imath}$ & $m$ & ? & $p$ & $p$ & $\hat{\imath}$ & $p$ & $m$ \\
\hline 으 & $\varrho$ & 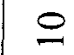 & 으 & 은 & $\stackrel{\varrho}{\circ}$ & 은 & $\stackrel{\varrho}{\circ}$ & 음 & 으 & 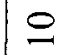 & 음 & 음 & 음 & 으 & $\varrho$ & $\stackrel{ }{-}$ & $\varrho$ \\
\hline$x$ & $x$ & $x$ & $x$ & $x$ & $x$ & $x$ & $x$ & $x$ & $x$ & $x$ & $x$ & $x$ & $x$ & $x$ & $x$ & $x$ & $x$ \\
\hline I & $\pi$ & 8 & ت & $\hat{m}$ & 2 & Ð & $\hat{m}$ & 9 & Z & $\hat{m}$ & 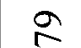 & $\Xi$ & $\hat{m}$ & 2 & $\Xi$ & $\hat{n}$ & $\frac{1}{2}$ \\
\hline$\stackrel{d}{d}$ & $\pi$ & ㄱ. & 志 & $\frac{m}{N}$ & 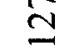 & $\underset{\sim}{\sim}$ & $\bar{N}$ & $\simeq$ & $\underset{\sim}{\sim}$ & $\vec{N}$ & ㄱ & $\stackrel{f}{\sim}$ & $\frac{m}{N}$ & 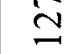 & $\underset{\sim}{\mathbb{J}}$ & $\pi$ & $\simeq$ \\
\hline- & - & - & - & - & - & - & - & - & - & - & - & - & $\dot{-}$ & $\dot{-}$ & - & - & - \\
\hline 11 & $\|$ & $\|$ & $\|$ & $\|$ & $\|$ & $\|$ & II & $\|$ & II & $\|$ & $\|$ & $\|$ & $\|$ & II & $\|$ & $\|$ & 11 \\
\hline${ }^{*} \sim$ & ${ }^{*} \sim$ & ${ }^{*} \infty$ & ${ }^{*}-$ & $*$ & ${ }^{*} \sim$ & ${ }^{*}-$ & ${ }^{*}-$ & ${ }^{*}+$ & ${ }^{*}+$ & ${ }^{*} \sim$ & ${ }^{*} \sim$ & *w & $*$ & ${ }^{*}-$ & ${ }^{*}-$ & ${ }^{*}-$ & $*$ \\
\hline
\end{tabular}

\begin{tabular}{|c|c|c|c|c|c|c|c|c|c|c|c|c|c|c|c|c|c|c|}
\hline \multicolumn{19}{|c|}{$c_{\circ}=0.65$} \\
\hline \multicolumn{9}{|c|}{ Mesh Size $=31 \times 31$} & \multicolumn{10}{|c|}{ Mesh Size $=20 \times 20$} \\
\hline \multicolumn{3}{|c|}{$\begin{array}{c}\text { Shallow Quench } \\
0.715 \text { to } 0.7\end{array}$} & \multicolumn{3}{|c|}{$\begin{array}{l}\text { Deep Quench } \\
0.715 \text { to } 0.63\end{array}$} & \multicolumn{3}{|c|}{$\begin{array}{l}\text { Deeper Quench } \\
0.715 \text { to } 0.525\end{array}$} & \multicolumn{3}{|c|}{$\begin{array}{c}\text { Shallow Quench } \\
0.715 \text { to } 0.7\end{array}$} & \multicolumn{4}{|c|}{$\begin{array}{l}\text { Deep Quench } \\
0.715 \text { to } 0.63\end{array}$} & \multicolumn{3}{|c|}{$\begin{array}{l}\text { Deeper Quench } \\
0.715 \text { to } 0.525\end{array}$} \\
\hline \multicolumn{19}{|c|}{ Diffusion } \\
\hline 1000 & 5000 & 8000 & 1000 & 5000 & 800 & 100 & 500 & 800 & 1000 & 5000 & 800 & 100 & & 000 & 8000 & 1000 & 5000 & 8000 \\
\hline 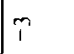 & $m$ & $\vec{\imath}$ & $\uparrow$ & ? & $\hat{\imath}$ & $m$ & $\uparrow$ & r & 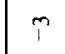 & $?$ & $\uparrow$ & $\uparrow$ & $?$ & $?$ & $m$ & m & r & $\hat{\imath}$ \\
\hline 으 & $\stackrel{\varrho}{=}$ & $\stackrel{0}{-}$ & 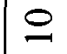 & 음 & $\stackrel{\circ}{-}$ & $\subseteq$ & $S$ & $\underline{0}$ & $\underline{0}$ & $\underline{\varrho}$ & $\stackrel{\varrho}{=}$ & $\stackrel{0}{-}$ & & 은 & $\underline{\underline{1}}$ & $\stackrel{-}{-1}$ & $\stackrel{-}{-}$ & $\stackrel{ }{=}$ \\
\hline$x$ & $x$ & $\times$ & $\times$ & $x$ & $x$ & $x$ & $x$ & $\times$ & $x$ & $x$ & $\times$ & $x$ & & $x$ & $x$ & $x$ & $x$ & $x$ \\
\hline 守 & $\underline{m}$ & $\frac{9}{2}$ & ষ্ঠ & $\tilde{m}$ & $\frac{9}{\pi}$ & $\Xi$ & ת & $\frac{9}{2}$ & $\stackrel{8}{g}$ & $\tilde{m}$ & $\frac{9}{\pi}$ & \& & & $\bar{m}$ & $\frac{9}{2}$ & $\stackrel{Ð}{\ominus}$ & $\hat{m}$ & $\frac{9}{2}$ \\
\hline 4 & ? & 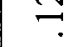 & ㄴ. & ? & $\because$ & r & c & 1 & ก & ? & $\because$ & 0 & & r & $\because$ & ก. & $\vec{\sim}$ & 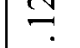 \\
\hline $\overrightarrow{\|}$ & $\vec{\pi}$ & $\overrightarrow{\|}$ & $\overline{11}$ & $\overrightarrow{\|}$ & $\overrightarrow{I I}$ & ॥ & 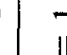 & $\bar{I}$ & 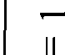 & $\overline{\|}$ & $\bar{I}$ & $\bar{I}$ & & $\vec{u}$ & $\vec{\pi}$ & $\vec{I}$ & $\bar{\pi}$ & $\vec{u}$ \\
\hline$*^{\prime \prime}$ & $*$ & ${ }^{*}$ & ${ }^{*}$ & ${ }^{*}$ & ${ }^{\prime \prime}$ & * & 11 & * & * & * & * & * & & II & * & * & * & ${ }_{*}^{\prime \prime}$ \\
\hline
\end{tabular}




\begin{tabular}{|c|c|c|c|c|c|c|c|}
\hline \multicolumn{10}{|c|}{$c_{0}=0.845$} \\
\hline \multicolumn{10}{|c|}{ Mesh Size $=31 \times 31$} \\
0.715 to 0.695
\end{tabular}

\begin{tabular}{|c|c|c|c|c|c|c|c|c|c|c|c|c|c|c|c|c|c|}
\hline \multicolumn{18}{|c|}{$c_{\circ}=0.89$} \\
\hline \multicolumn{9}{|c|}{ Mesh Size $=31 \times 31$} & \multicolumn{9}{|c|}{ Mesh Size $=20 \times 20$} \\
\hline \multicolumn{3}{|c|}{$\begin{array}{l}\text { Shallow Quench } \\
0.6795 \text { to } 0.6445\end{array}$} & \multicolumn{3}{|c|}{$\begin{array}{l}\text { Deep Quench } \\
0.6795 \text { to } 0.58\end{array}$} & \multicolumn{3}{|c|}{$\begin{array}{l}\text { Deeper Quench } \\
0.6795 \text { to } 0.525\end{array}$} & \multicolumn{3}{|c|}{$\begin{array}{l}\text { Shallow Quench } \\
0.6795 \text { to } 0.6445\end{array}$} & \multicolumn{3}{|c|}{$\begin{array}{c}\text { Deep Quench } \\
0.6795 \text { to } 0.58\end{array}$} & \multicolumn{3}{|c|}{$\begin{array}{l}\text { Deeper Quench } \\
0.6795 \text { to } 0.525\end{array}$} \\
\hline \multicolumn{18}{|c|}{ Diffusion } \\
\hline 1000 & 5000 & 8000 & 1000 & 5000 & 8000 & 1000 & 5000 & 8000 & 1000 & 5000 & 8000 & 1000 & 5000 & 8000 & 1000 & 5000 & 8000 \\
\hline$m$ & $\hat{\imath}$ & $\hat{\imath}$ & $\hat{\imath}$ & $\hat{\imath}$ & ? & $p$ & $?$ & $p$ & 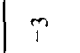 & $\uparrow$ & $\uparrow$ & p & $\vec{\imath}$ & $\uparrow$ & $p$ & 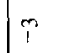 & $\uparrow$ \\
\hline 은 & $\stackrel{\varrho}{=}$ & $\varrho$ & $\underline{\varrho}$ & 으 & $\underline{\underline{O}}$ & $\underline{0}$ & 음 & $\varrho$ & $\underline{\underline{ }}$ & $\underline{\underline{O}}$ & $\underline{\varrho}$ & $\stackrel{0}{1}$ & $\underline{0}$ & $\varrho$ & $\varrho$ & $\varrho$ & $\underline{0}$ \\
\hline$x$ & $\times$ & $\times$ & $x$ & $\times$ & $x$ & $x$ & $\times$ & $\times$ & $\times$ & $\times$ & $\times$ & $x$ & $x$ & $x$ & $x$ & $x$ & $\times$ \\
\hline ப & $\hat{n}$ & 9 & $\Xi$ & $\hat{n}$ & 2 & $\Xi$ & $\hat{m}$ & 2 & J & $\hat{m}$ & 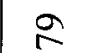 & ઇ & $\bar{m}$ & 9 & $\Xi$ & $\bar{m}$ & 8 \\
\hline$\stackrel{+}{\sim}$ & $\bar{\tau}$ & $\stackrel{ }{\beth}$ & さ & $\vec{\sim}$ & 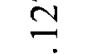 & ণ & $\overrightarrow{\widetilde{N}}$ & 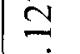 & $\stackrel{\dot{J}}{\triangleleft}$ & $\bar{N}$ & 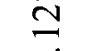 & $\stackrel{\text { J }}{\sim}$ & $\bar{N}$ & $\simeq$ & $\underset{\sim}{\sim}$ & $\bar{N}$ & $\simeq$ \\
\hline- & $-i$ & $\dot{-}$ & - & - & - & $\dot{-}$ & - & $\dot{-}$ & $\dot{-}$ & - & - & $-\dot{ }$ & - & $\dot{-}$ & $\dot{-}$ & - & $\dot{-}$ \\
\hline$\|$ & $\|$ & II & $\|$ & $\|$ & $\|$ & $\|$ & $\|$ & II & $\|$ & $\|$ & 11 & $\|$ & $\|$ & $\|$ & $\|$ & $\|$ & $\|$ \\
\hline * & ${ }^{*}-$ & ${ }^{*} \sim$ & ${ }^{*}+$ & * & ${ }^{*}-$ & ${ }^{*}+$ & $*$ & $*$ & " & ${ }^{*}+$ & ${ }^{*}-$ & ${ }^{*}-$ & $*^{*}$ & $*$ & ${ }^{*}-$ & ${ }^{*}-$ & ${ }^{*} \sim$ \\
\hline
\end{tabular}




\begin{tabular}{|c|c|c|c|c|c|c|c|c|c|c|c|}
\hline \multicolumn{12}{|c|}{$c_{\mathrm{o}}=0.93$} \\
\hline \multicolumn{6}{|c|}{ Mesh Size $=31 \times 31$} & \multicolumn{6}{|c|}{ Mesh Size $=20 \times 20$} \\
\hline \multicolumn{3}{|c|}{$\begin{array}{l}\text { Shallow Quench } \\
0.6795 \text { to } 0.6445\end{array}$} & \multicolumn{3}{|c|}{$\begin{array}{c}\text { Deep Quench } \\
0.6795 \text { to } 0.525\end{array}$} & \multicolumn{3}{|c|}{$\begin{array}{l}\text { Shallow Quench } \\
0.6795 \text { to } 0.6445\end{array}$} & \multicolumn{3}{|c|}{$\begin{array}{c}\text { Deep Quench } \\
0.6795 \text { to } 0.525\end{array}$} \\
\hline \multicolumn{12}{|c|}{ Diffusion } \\
\hline 1000 & 5000 & 8000 & 1000 & 5000 & 8000 & 1000 & 5000 & 8000 & 1000 & 5000 & 8000 \\
\hline$\frac{\hat{1}}{\stackrel{0}{x}}$ & $\frac{p}{x}$ & $\frac{\mathfrak{T}_{0}}{0}$ & $\frac{i}{x}$ & $\frac{1}{x}$ & $\frac{\hat{p}}{x}$ & $\frac{\hat{p}}{x}$ & $\frac{\hat{1}}{x}$ & $\frac{i}{x}$ & $\frac{\hat{c}}{x}$ & $\frac{\hat{r}}{x}$ & $\frac{\hat{p}}{x}$ \\
\hline$\underset{\sim}{\stackrel{+}{\sim}}$ & $\frac{\vec{n}}{\sim}$ & $\underset{\Xi}{\stackrel{\Xi}{\Xi}}$ & 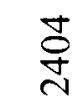 & $\stackrel{\vec{n}}{\vec{N}}$ & $\stackrel{\text { Iิ }}{\underline{\Xi}}$ & $\underset{\sim}{\stackrel{+}{\sim}}$ & $\stackrel{\vec{a}}{\vec{\sim}}$ & 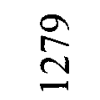 & $\underset{\sim}{\stackrel{+}{+}}$ & 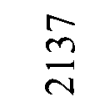 & 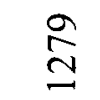 \\
\hline- & $\dot{-}$ & $\dot{-}$ & $-\dot{ }$ & $\dot{-}$ & - & $-i$ & $-\dot{-}$ & $-\dot{-}$ & $-\dot{ }$ & $-\dot{ }$ & $\dot{-}$ \\
\hline ." & * & . & ${ }^{*}$ & * & ${ }_{*}^{*}$ & * & ." & * & * & * & ." \\
\hline${ }^{*}-2$ & ${ }^{*}-$ & ${ }^{*}-$ & 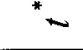 & ${ }^{*}-$ & ${ }^{*}-$ & $*$ & ${ }^{*}-$ & 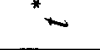 & * - & ${ }^{*}-$ & ${ }^{*}-$ \\
\hline
\end{tabular}


Numerical parameter values

\begin{tabular}{|lcc|}
\hline Parameter & Value & Reference \\
\hline$N_{1}$ & 1 & This study \\
$N_{2}$ & 10 & This study \\
$\psi$ & 1 & 45 \\
$M\left(\mathrm{~m}^{5} \mathrm{~J}^{-1} \mathrm{~s}^{-1} \times 10^{-23}\right)$ & 1 & 45 \\
$\theta(\mathrm{K})$ & 300 & 45 \\
$\chi$ & 1.1667 & This study \\
$L\left(\mathrm{~m}^{\times} \times 10^{-6}\right)$ & 5 & This study \\
$U\left(\mathrm{~m}^{3} \mathrm{segment}^{-1} \times 10^{-27}\right)$ & 1 & 45 \\
$k_{B}\left(\mathrm{JK}^{-1} \times 10^{-23}\right)$ & 1.3806504 & Universal Constant \\
$\kappa\left(\mathrm{Jm}^{-1} \times 10^{-7}\right)$ & 1 & 45 \\
$D\left(\mathrm{~m}^{2} \mathrm{~s}^{-1} \times 10^{-10}\right)$ & 1.21 & This study \\
$t\left(\mathrm{~s} \times 10^{3}\right)$ & $1-10$ & This study \\
\hline
\end{tabular}

